\title{
Yannis Kakridis
}

\section{Wortbildung und Kategorisierung am Beispiel der desubstantivischen Wortbildung des Russischen}

Verlag Otto Sagner München · Berlin · Washington D.C.

Digitalisiert im Rahmen der Kooperation mit dem DFG-Projekt „Digi20“

der Bayerischen Staatsbibliothek, München. OCR-Bearbeitung und Erstellung des eBooks durch den Verlag Otto Sagner:

http://verlag.kubon-sagner.de

() bei Verlag Otto Sagner. Eine Verwertung oder Weitergabe der Texte und Abbildungen, insbesondere durch Vervielfältigung, ist ohne vorherige schriftliche Genehmigung des Verlages unzulässig. 


\title{
SLAVISTISCHE BEITRÄGE
}

\author{
Begründet von \\ Alois Schmaus \\ Herausgegeben von \\ Peter Rehder
}

\author{
Beirat:
}

Tilman Berger - Walter Breu - Johanna Renate Döring-Smimov

Walter Koschmal · Ulrich Schweier - Miloš Sedmidubský - Klaus Steinke

\author{
BAND 376
}

\section{VERLAG OTT(O) SAGNER \\ MÜNCHEN 1999}

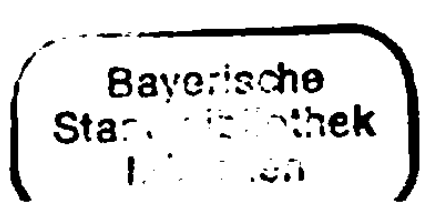




\section{Yannis Kakridis}

\section{Wortbildung und Kategorisierung am Beispiel \\ der desubstantivischen Wortbildung des Russischen}

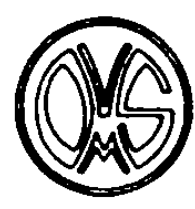

VERLAG OTTO SAGNER

MÜNCHEN 1999 
ISBN 3-87690-727-6

(C) Verlag Otto Sagner. München 1999

Abteilung der Firma Kubon \& Sagner

D-80328 München

Gedruckt auf alterungshestïndigem Papier

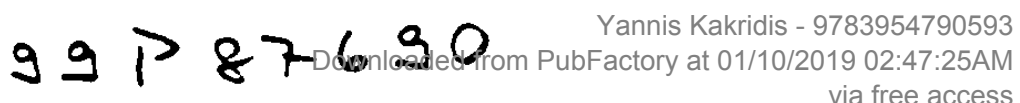


Citius emergit veritas ex errore quam ex confusione

FRANCIS BACON 


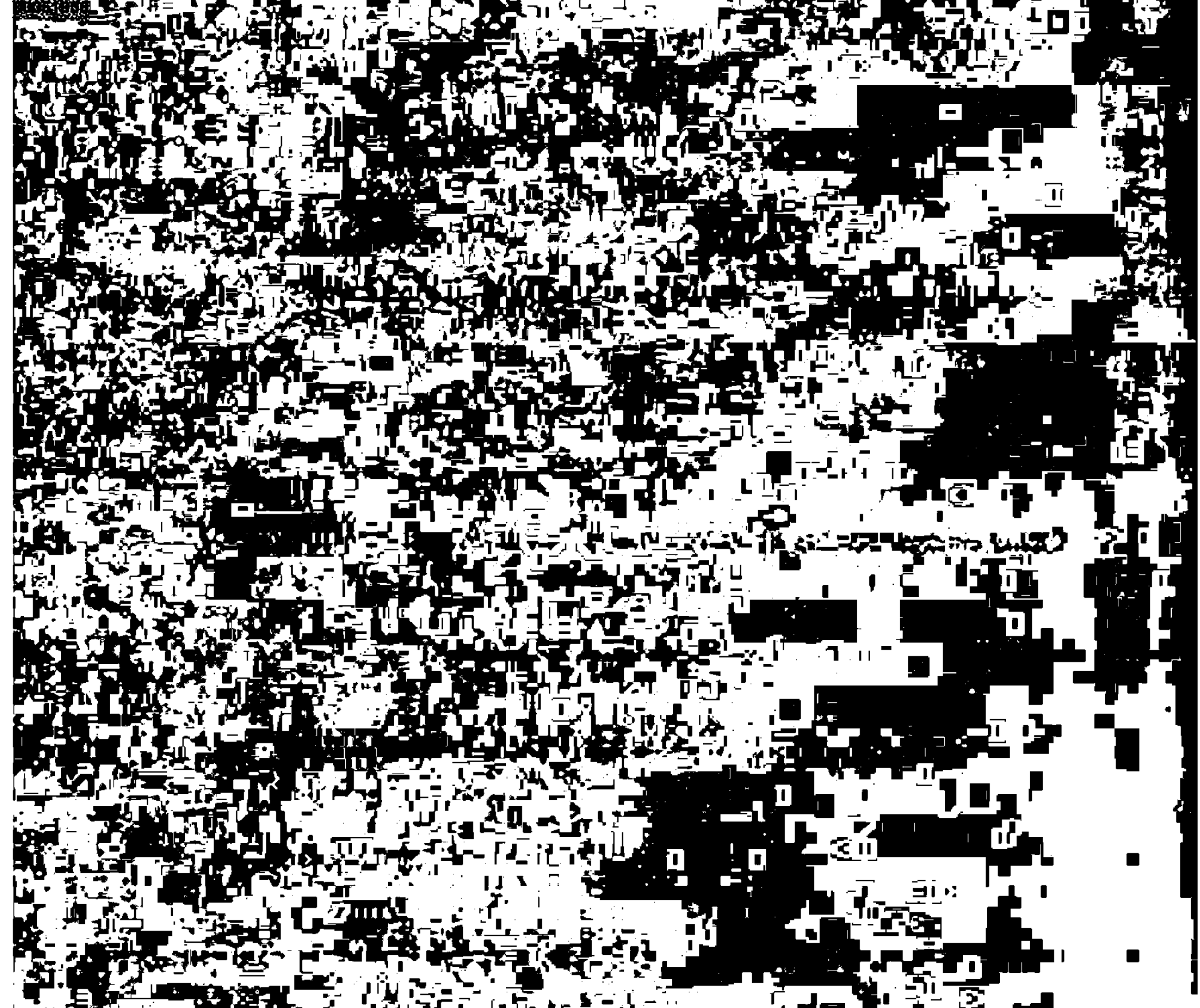

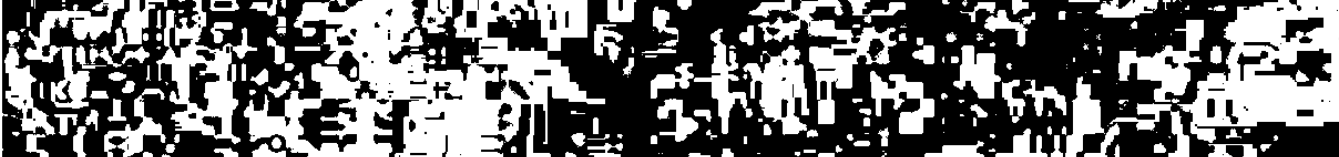

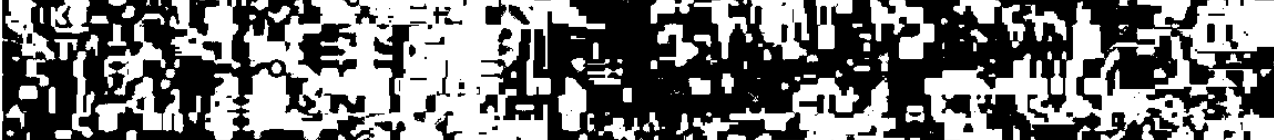

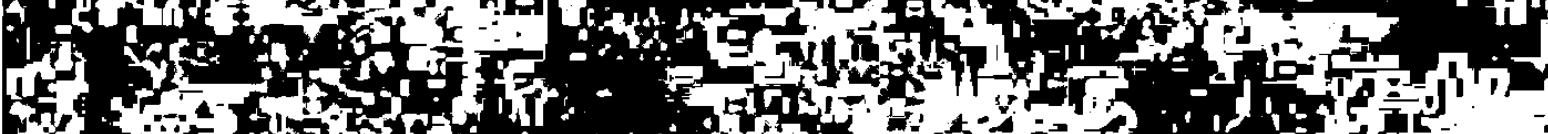

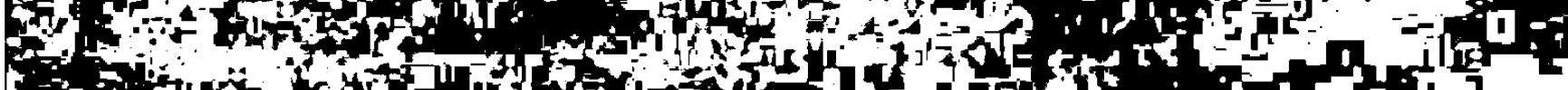

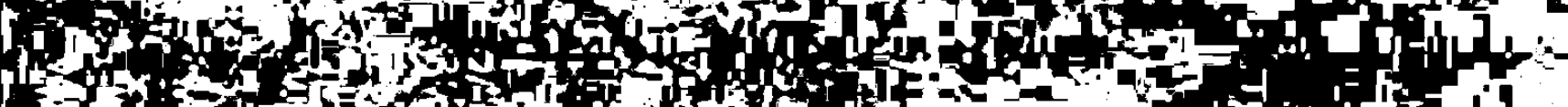

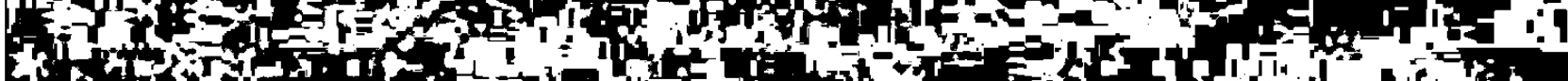

if

1 is

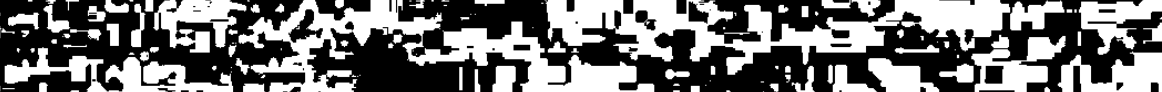

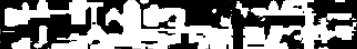

tor

(1)

K $=17$

$\infty$

the

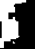

A

-

of

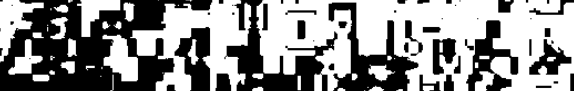

in ind :

Antsing

$3=-1$

$=1+y$

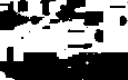




\section{VORWORT}

Dies ist die gestraffte und grundlegend überarbeitete Fassung einer Arbeit, die im Wintersemester 1995 von der Philosophischen Fakultät der Universität Bonn als Habilitationsschrift angenommen wurde. Bei ihrer Abfassung habe ich von folgenden Personen Hilfe und Unterstützung erfahren: G. Abakumkin, G. Altmann, D. Christians, D. Frick, R.M. Frumkina, E. Kankeleit, E.G. Krejdlin, H. Keipert, H. Kniffka, W. Lenders, V. N. Maskadynja, A.V. Mixeev, M. Rammelmeyer, K. Reichl, Chr. Schmitt, H. Siemens, A. Timberlake, N.A. Tixomirova, E.A. Zemskaja und V.M. Živov. Ihnen allen sei aufrichtig gedankt. Mein besonderer Dank gilt A. Leschhorn und $\mathbf{M}$. Černyševa, die das gesamte Typoskript gelesen und mit wertvollen Anmerkungen versehen haben. Herm Prof. P. Rehder danke ich für die Aufnahme der Arbeit in die Reihe der "Slavistischen Beiträge". Danken möchte ich schließlich der Deutschen Forschungsgemeinschaft, die mir ermöglicht hat, während eines zweimonatigen Forschungsaufenthaltes in Moskau das Ausgangsmaterial für meine Untersuchung zu sammeln und schwer zugängliche Publikationen einzusehen. Für verbliebene Mängel trage allein ich die Verantwortung.

Der Leser sollte sich über den experimentellen Charakter dieser Arbeit im klaren sein. Mein wichtigstes Anliegen war, Gegensätze nicht zu verwischen, sondern auf die Spitze zu treiben, die positiven, aber vor allem auch die negativen Konsequenzen methodischer Grundentscheidungen in möglichst scharfer Form herauszuarbeiten, und jede Art von eklektizistischer Verwässerung zu vermeiden. Ich bin überzeugt, daß erst eine solche Zuspitzung der Widersprüche die Voraussetzungen für deren wahre Versöhnung schafft, während der Eklektizismus bei allem scheinbaren Fortschritt in der Materialerfassung letztlich doch vor dem antinomischen Charakter der Sprache kapitulieren muß. Es sei aber auch betont, daß die Untersuchung, die auf diese Weise entstanden ist, nur den Status einer negativen Vorarbeit beanspruchen darf; Leser, die Gedankenarbeit ohne unmittelbare praktische Ergebnisse für überflüssigen Luxus halten, sollten deshalb dieses Buch spätestens jetzt aus der Hand legen.

Bonn, im November 1998

Yannis Kakridis 


\section{INHALTSVERZEICHNIS}

Einleitung: Syntagmatische und paradigmatische Wortbildungslehre

1. Wörter für Sachen

1.1 Konkrete Substantive: Wörter oder Sachen?

1.2 Grundfragen der natürlichen Kategorisierung

1.2.1 Horizontale und vertikale Profilierung natürlicher Kategorien

1.2.2 Qualitative Aspekte der Struktur natürlicher Kategorien

1.3 Zum Material der vorliegenden Untersuchung

2 Vom Ableitungspaar zum Wortbildungsparadigma

2.1 Das Korpus: ein Metawörterbuch

2.2 Aufstellung der Wortbildungsparadigmen

2.2.1 Formale Beziehungen zwischen Lexemen

2.2.2 Inhaltliche Beziehungen zwischen Lexemen

2.2.3 Zusammenfassung der Lexeme zu Wortfamilien

2.2.4 Zusammenfassung und Aufspaltung von Lexemen

2.2.5 Definition des Wortbildungsparadigmas

3 Paradigmatische Wortbildungsanalyse: theoretische Fragen

3.1 Analyseprinzipien

3.4 Die Behandlung der Beziehungsadjektive 
4.1 Analyse der Wortbildungsparadigmen der Kategorie Frukty (Obst)

4.1.1. Zum Bestand der Kategorie

4.1.2 Zum Bestand der Wortbildungsparadigmen 138

4.1.3 Das abstrakte Wortbildungsparadigma der Kategorie Frukty (Obst) 138

4.1.4 Ausgewählte Wortbildungsparadigmen

4.2 Analyse der Wortbildungsparadigmen der Kategorie Ovošč (Gemüse) 152

4.2.1 Zum Bestand der Kategorie

4.2.2 Zum Bestand der Wortbildungsparadigmen 152

4.2.3 Das abstrakte Wortbildungsparadigma der Kategorie Ovošči (Gemüse) 153

4.2.4 Ausgewählte Wortbildungsparadigmen

4.3 Analyse der Wortbildungsparadigmen der Kategorie Pticy (Vögel)

4.3.1 Zum Bestand der Kategorie und der Wortbildungsparadigmen

4.3.2 Das abstrakte Wortbildungsparadigma der Kategorie Pticy (Vögel)

4.3.3 Ausgewählte Wortbildungsparadigmen

4.4 Analyse der Wortbildungsparadigmen der Kategorie Posuda (Küchengeschirr und Besteck)

4.4.1 Zum Bestand der Kategorie

4.4.2 Zum Bestand der Wortbildungsparadigmen

4.4.3 Das abstrakte Wortbildungsparadigma der Kategorie Posuda (Küchengeschirr und Besteck)

4.4.4. Ausgewählte Wortbildungsparadigmen

4.5 Analyse der Wortbildungsparadigmen der Kategorie Sredstva peredviženija (Fortbewegungsmittel)

Anhang 1: Produktionsnormen zu fünf Kategorien konkreter Substantive des Russischen (Exemplar-Dominanz)

Anhang 2: Kategoriennormen nach V.N. MASKADYNJA (1987) 


\section{EINLEITUNG: SYNTAGMATISCHE UND PARADIGMATISCHE WORT- BILDUNGSLEHRE}

Diese Untersuchung ist der Analyse und Kategorisierung abgeleiteter Wörter des Russischen gewidmet; ihr Anliegen kann in zwei Fragen zusammengefaßt werden:

a) welchen Anteil hat die Wortbildungsbedeutung an der lexikalischen Bedeutung des abgeleiteten Wortes?

b) welches sind die semantischen Bausteine des Wortbildungssystems einer Sprache (ihre Wortbildungskategorien)?

Wie sich bald zeigen wird, sind a) und b) nur verschiedene Aspekte ein und derselben Fragestellung. Zur Einführung in diese Fragestellung mögen folgende Beispiele dienen:

oxota 'poiski, vysleživanie zverej, ptic s cel'ju umerščvlenija (na kogo) ili lovli (za kem)' 'Jagd' $\rightarrow$ oxotnik 'čelovek, k-ryj zanimaetsja oxotoj' 'Jäger'

montaž 'Verbalsubstantiv zu montirovat" 'montieren' $\rightarrow$ montažnik 'specialist po montažu konstrukcij' 'Spezialist für Montagearbeiten (am Bau)'

zavist' 'čuvstvo dosady, vyzvannoe blagopolučiem, uspexom drugogo' 'Neid' $\rightarrow$ zavistnik 'zavistlivyj čelovek' 'neidischer Mensch, Neider'

ljubov' 'čuvstvo samootveržennoj i glubokoj privjazannosti, serdečnogo vlečenija' 'Liebe' $\rightarrow$ ljubovnik 'mužčina, k-ryj naxoditsja v svjazi s ženščinoj, ne sostoja $s$ nej $v$ oficial'nom brake' 'Liebhaber (einer Frau)',

In allen vier Paaren ist jeweils das zweite Wort vom ersten abgeleitet; sie bilden ein Ableitungs- oder Wortbildungspaar. Dies bedeutet, daß das erste Wort (das ableitende Wort) im zweiten (dem abgeleiteten) formal und inhaltlich enthalten ist: sowohl die Ausdrucks- als auch die Inhaltsseite des ableitenden Wortes sind in der Ausdrucksbzw. der Inhaltsseite des abgeleiteten Wortes enthalten. Auf der Inhaltsseite ist diese Beziehung dadurch festzustellen, daß das abgeleitete Wort mit Hilfe des ableitenden definiert werden kann. (Im Falle von ljubovnik ist die inhaltliche Beziehung durch das Substantiv svjaz' 'Beziehung' vermittelt, cf. die Erklärung des letzteren in SVE: 'ljubovnye otnošenija, sožitel'stvo'.)

1 Bedeutungsangaben stammen hier und im folgenden von einem der in der Bibliographie (Abschnitt A) genannten Wörterbücher; bei Wörtern, die in den russisch-deutschen Wörterbüchern nicht belegt sind, wurde der deutschen Bedeutungsangabe die russische zugrundegelegt. 
Sowohl auf der Ausdrucksseite als auch auf der Inhaltsseite ist also das abgeleitete Wort komplexer als das ableitende: seine Ausdrucksseite besteht aus der Ausdrucksseite des ableitenden Wortes und dem Wortbildungsformanten; seine Inhaltsseite enthält außer der Inhaltsseite des ableitenden Wortes zusätzliche semantische Komponenten, die seine Wortbildungsbedeutung ausmachen.

Der Vergleich der angeführten Ableitungspaare zeigt allerdings, daß der Zuwachs auf der Inhaltsseite recht unterschiedlich ausfallen kann: man kann alles mögliche montieren, aber nur der Monteur von (Bau)konstruktionen ist ein montažnik; und nicht jede Liebesbeziehung zwischen Mann und Frau läßt den Mann als ljubovnik erscheinen (es kann auch ihr Ehegatte sein). Es liegt nahe, solche semantischen Komponenten wie 'auf dem Bau' oder 'außerehelich' nicht der Wortbildungsbedeutung zuzuschlagen, sondern als individuelle Besonderheiten der lexikalischen Einheiten montažnik und ljubovnik anzusehen, zumal Entsprechendes bei oxotnik und zavistnik nicht auszumachen ist. Folgt man dieser Deutung, so würden die entsprechenden Bedeutungselemente die idiomatische Komponente der Inhaltsseite des abgeleiteten Wortes bilden.

Die Inhaltsseite des abgeleiteten Wortes würde also aus drei Zonen bestehen, wie folgendes Schema verdeutlicht:

\begin{tabular}{|l|l|}
\hline Inhaltsseite des abgeleiteten Wortes & 'montažnik' \\
\hline Wortbildungsbedeutung & 'specialist po ... \\
\hline Inhaltsseite des ableitenden Wortes & $\ldots$ montažu ... \\
\hline $\begin{array}{l}\text { idiomatische Bedeutungskom- } \\
\text { ponenten }\end{array}$ & $\ldots$ konstrukcij' \\
\hline
\end{tabular}

$\mathrm{Zu}$ den zentralen Aufgabenstellungen einer Wortbildungslehre, die semantischen Fragestellungen nicht aus dem Wege geht, gehört die Aufstellung von Kriterien, nach denen in der Inhaltsseite des abgeleiteten Wortes die Wortbildungsbedeutung von den idiomatischen Bedeutungskomponenten getrennt werden kann. Um beim Beispiel montažnik zu bleiben: gehört die Bedeutungskomponente 'professionell', die in specialist 'Spezialist, Facharbeiter' enthalten ist, wirklich der Wortbildungsbedeutung von montažnik an -- oder sollte man sie nicht vielmehr als idiomatisch einstufen? Man beachte, daß die beiden Analysen zu je unterschiedlichen Kategorisierungsentscheidungen führen: entscheidet man sich für die erste Alternative, so gehört montažnik einer anderen Wortbildungskategorie an als zavistnik, dessen Wortbildungsbedeutung die Komponente 'professionell' nicht aufweist; entscheidet man sich für die zweite, so kann man montażnik und zavistnik derselben Wortbildungskategorie 
zuschlagen. Montažnik ist übrigens nicht die einzige Berufsbezeichnung, die man von montaž ableiten kann; cf. die Neologismen

montažer 'specialist po montažu ( $v$ kinematografii, fotografii)' 'Spezialist für Montagearbeiten (in der Film- und Photoindustrie)'

montažist 'specialist, montirujuščij pečatnyj nabor, stereotipy, kliše' 'Spezialist für Montagearbeiten (im Druck- und Medienwesen)'

Reicht die Gemeinsamkeit der semantischen Komponente 'professionell' aus, um montažnik, montažer und montažist (von den unterschiedlichen Wortbildungsformanten einmal abgesehen) als Mitglieder derselben Wortbildungskategorie auszuweisen?

Ähnliche Fragen stellen sich bei sehr vielen abgeleiteten Wörtern. Man vergleiche etwa in dieser Hinsicht glazastyj und bel'mastyj:

bel'mo 'belovatoe pjatno na rogovoj oboločke glaza, obrazovavšeesja posle ee vospalenija ili pri povreždenii glaza' 'weißer Star' -- bel'mastyj 'imejuščij bel'mo (o glaze); imejuščij bel'mo na glazu (o čeloveke)'2 'starblind (Auge, Mensch)'

glaz 'organ zrenija, a takže samo zrenie' 'Auge' -- glazastyj 's bol'šimi glazami ili s glazami navykate; s ostrym zreniem, zorkij' 'großäugig, mit scharfen Augen'

Gehört die Bedeutungskomponente 'auffällig groß' in glazastyj der Wortbildungsbedeutung dieses Wortes an? Wie steht es um die Bedeutungskomponente 'sportlich' in velodrom 'sportivnoe sooruženie dlja velosipednyx gonok i trenirovok' 'Radrennbahn' oder 'Menschen' in molodež' 'molodoe pokolenie, molodye ljudi' 'Jugend'? Muß man sich in diesen und ähnlichen Fällen mit der intuitiven Abgrenzung von Wortbildungsbedeutung und idiomatischen Bedeutungskomponenten begnügen, wie sie etwa bei UluXanov 1977, ERMaKova 1984 oder in mehreren Definitionen bei MeL'ĆUK 1993-94, Bd. 1 (1.5, pp.151-153; I.34, I.35, pp. 311-315; cf. 290-293 und Bd. 2, 311-403) vorausgesetzt wird, oder kann man dafür explizite Kriterien aufstellen?

Im folgenden sollen zwei Methoden vorgestellt werden, mit denen man an die Beantwortung der Frage nach der Grenze zwischen Wortbildungsbedeutung und idiomatischer Bedeutung im abgeleiteten Wort (nach der Grenze zwischen Wortbildung und Lexikologie) herangehen kann: die syntagmatische bzw. die paradigmatische Methode der Wortbildungslehre. Um das Wesentliche gleich vorwegzunehmen: bei der syntagmatischen Methode steht das Verhältnis zwischen montaž und

${ }^{2}$ So BAS2; die Definition von BAS1 'imejuščij bol'šoe bel'mo na glazu' wurde in der zweiten Auflage zu recht korrigiert. 
montažnik, bei der paradigmatischen das Verhältnis zwischen montažnik, montažer und montažist im Mittelpunkt der Betrachtung.

Die syntagmatische Wortbildungslehre geht von den Ableitungspaaren aus und versucht, sie auf Grund formaler und inhaltlicher Gemeinsamkeiten zu Einheiten höherer Abstraktionsstufen zusammenzufassen. ${ }^{3}$ Wir geben wiederum einige (möglichst triviale) Beispiele, wobei aus Gründen der Einfachheit die Inhaltsseite jetzt nicht mehr durch die russische Wörterbuchdefinition, sondern ausschließlich durch die deutsche Übersetzung repräsentiert wird:

$\begin{array}{ll}\text { čitat' 'lesen' } & \text { čitatel' 'Leser' } \\ \text { mečtat' 'träumen' } & \begin{array}{l}\text { mečtatel' 'Träumer' } \\ \text { osvoboditel 'Befreier' } \\ \text { osvobodit' 'befreien' }\end{array} \\ \text { stroit' 'bauen' } & \begin{array}{l}\text { stroitel' 'Bauarbeiter' } \\ \text { issledovat' 'erforschen' }\end{array} \text { issledovatel' 'Forscher' } \\ \text { izdat' 'herausgeben' } & \text { izdatel' 'Herausgeber' }\end{array}$

Beim Vergleich dieser Wortpaare stellt man fest:

a) eine Parallelität der Beziehung zwischen den Ausdrucksseiten des ableitenden und des abgeleiten Wortes (ihrer formalen Beziehung) und

b) eine Parallelität der Beziehung ihrer Inhaltsseiten (ihrer inhaltlichen Beziehung).

Man kann diese Parallelität zum Anlaß nehmen, diese (und andere) Wörter zu einem Wortbildungstyp zusammenzufassen. Ein solcher Wortbildungstyp stellt eine Einheit von Wortbildungsformanten (in unserem Fall das Suffix -tel) und Wortbildungsbedeutung dar; letztere erhält man, wenn man aus den Inhaltsseiten der abgeleiteten Wörter jene Bedeutungselemente streicht, die nicht allen abgeleiteten Wörtern gemeinsam sind, d.h. die Inhaltsseiten der ableitenden Wörter und die idiomatischen Bedeutungskomponenten. In unserem Fall könnte man diese Wortbildungsbedeutung etwa folgendermaßen umschreiben: 'Person, die [träumt, liest, ...]' (man beachte, daß die Verben sowohl eine habituelle als auch eine aktuelle Lesart zulassen; insofern diese Lesarten nicht bei allen Ableitungen identisch sind, fallen sie aus der Wortbildungsbedeutung heraus).

${ }^{3}$ Die Bezeichnung syntagmatisch findet ihre Rechtfertigung darin, daß die Beziehung zwischen den Gliedern des Ableitungspaares (montaž $\rightarrow$ montažnik) und die Beziehung zwischen den Bestandteilen des abgeleiteten Wortes (montažnik = montaž + nik) lediglich zwei Seiten derselben Medaille sind (HockETT 1954). 
Auch im Bereich der Wortbildung besteht zwischen der Ausdrucks- und der Inhaltsseite der sprachlichen Zeichen keine Isomorphie. So findet man im Russischen zahlreiche Ableitungspaare, bei denen die formale Beziehung zwischen dem ableitenden und dem abgeleiteten Wort mit derjenigen zwischen čitat' und citatel', mečtat' und mečtatel' etc. übereinstimmt, die inhaltliche jedoch nicht:

$\begin{array}{ll}\text { vytrezvit' 'ernüchtern' } & \text { vytrezvifel 'Ernüchterungszelle' } \\ \text { gasit' 'löschen' } & \text { gasitel' 'Feuerlöscher' } \\ \text { izmerit' 'messen' } & \text { izmeritel' 'Meßapparat' } \\ \text { dubit' 'gerben' } & \text { dubitel' 'Gerbstoff' } \\ \text { poglotit' 'verschlucken' } & \text { poglotitel' 'Absorptionsmittel' }\end{array}$

Diese Ableitungen bezeichnen keine Personen, sondern Gegenstände, die in das Verbalgeschehen der Ableitungsbasis auf die unterschiedlichste Art und Weise eingehen: als räumliche Umgebung (vytrezvitel), als Werkzeug (gasitel) oder Hilfsstoff (dubitel). -- Andererseits lassen sich leicht Ableitungspaare finden, bei denen die inhaltliche, nicht jedoch die formale Beziehung zwischen ableitendem und abgeleitetem Wort mit der zwischen čitat' und čitatel', mečtat' und mečtatel' etc. übereinstimmt:

\begin{tabular}{|c|c|}
\hline $\begin{array}{l}\text { sutit' 'scherzen' } \\
\text { tancevat' 'tanzen' }\end{array}$ & $\begin{array}{l}\text { sutnik 'Witzbold' } \\
\text { tancovščik 'Tänzer' }\end{array}$ \\
\hline $\begin{array}{l}\text { l'stit' 'schmeicheln' } \\
\text { tkat' 'weben' }\end{array}$ & $\begin{array}{l}\text { l'stec 'Schmeichler' } \\
\text { tkać 'Weber' }\end{array}$ \\
\hline
\end{tabular}

Diese Asymmetrie zwischen der Ausdrucks- und der Inhaltsseite der Wortbildungsmittel hat zur Folge, daß man die Wortbildungsbedeutungen einer Sprache (in unserem Fall des Russischen) nicht einfach dadurch ermitteln kann, daß man alle Wörter mit einem bestimmten Formanten sammelt und auf ihren gemeinsamen Bedeutungsinhalt hin untersucht. Es ist notwendig, auf eine höhere Abstraktionsstufe zu steigen, indem man entweder auf die formale oder auf die inhaltliche Parallelität in den einzelnen Ableitungspaaren verzichtet.

Geht man vom Wortbildungsformanten aus, so verzichtet man auf das Kriterium der inhaltlichen Parallelität zwischen den Ableitungen, d.h. auf die Identität der Wortbildungsbedeutung. Der weitaus größte Teil der Wortbildungsuntersuchungen folgt diesem bewährten Rezept: man sammelt alle abgeleiteten Wörter, die einen bestimmten Formanten aufweisen, um sie anschließend nach semantischen Gesichtspunkten einem oder mehreren Wortbildungstypen zuzuordnen (cf. das Wörterbuch von EFREMOVA 1996). Da zwischen der Ausdrucks- und der Inhaltsseite des sprachlichen Zeichens zwar Asymmetrie, jedoch keine vollständige Disparität besteht, führt dieses Verfahren in der Regel zu sinnvollen Ergebnissen. Der Dialektik von Idiomatik und Systematik kann man freilich auch auf diese Weise nicht entgehen: auch die Aus- 
drucksseite des abgeleiteten Wortes enthält nicht selten neben der Ausdrucksseite des ableitenden Wortes und dem Wortbildungsformanten eine idiomatische Komponente (man denke nur an das Problem der Interfigierung).

Man kann jedoch auch von den Wortbildungsformanten abstrahieren und die Ableitungspaare nach der Parallelität der inhaltlichen Beziehung zwischen ihren Gliedern zu Einheiten höherer Ordnung zusammenfassen; die traditionelle Bezeichnung für eine solche Einheit höherer Ordnung ist Wortbildungskategorie. ${ }^{4}$ So könnte man etwa auf Grund der inhaltlichen Parallelität zwischen čitat' und čitatel', šutit' und sutnik, l'stit' und l'stec etc. all diese Ableitungspaare zu einer Wortbildungskategorie zusammenfassen, deren Inhalt mit 'Person, die [liest, scherzt, schmeichelt...]' wiedergegeben werden könnte.

An dieser Stelle stößt die syntagmatische Methode der Wortbildungslehre auf eine unauflösbare Aporie: der Abstraktionsgrad von Wortbildungskategorien kann, da ja eine Anlehnung an die Ausdrucksseite der Wortbildungsmittel nicht statthaft ist, nur apriorisch, d.h. vom Wortbildungsstandpunkt aus willkürlich festgelegt werden. Zwischen den beiden Extrema einer radikalen Differenzierung, die für jedes Ableitungspaar eine eigene Wortbildungskategorie aufstellt, und einer ebenso radikalen Pauschalierung, die mit einer einzigen, allumfassenden Wortbildungskategorie auskäme, öffnet sich ein weites Spektrum von Lösungsmöglichkeiten. So könnte man auch in unserem Beispiel die Ableitungen citatel', šutnik, l'stec, issledovatel', tkač etc. auf zwei Wortbildungskategorien verteilen -- je nachdem, ob es sich bei ihnen um Berufsbezeichnungen handelt oder nicht; oder aber man könnte sie zusammen mit vytrezitel', dubitel', gasitel' und einer Vielzahl anderer Ableitungen zu einer Wortbildungskategorie zusammenfassen, deren Inhalt nunmehr ganz allgemein mit 'Entität [Person oder Gegenstand], die am [Lesen, Scherzen, Gerben, Löschen, ...] beteiligt ist' umschrieben werden müßte. Entsprechende Differenzierungs- bzw. Verallgemeinerungsmöglichkeiten ergeben sich auch in anderen Bereichen der Wortbildung; man denke etwa an die vielen unterschiedlichen Gesichtspunkte, nach denen Werkzeuge/ Apparaturen/Materialien sowie Räumlichkeiten/Behältnisse eingeteilt werden können. ${ }^{6}$

+ Die Termini Wortbildungstyp und Wortbildungskategorie haben sich unter dem Einfluß der Wortbildungstheorie von MILOŚ DoKULIL durchgesetzt: s. DOKULIL 1962: 68sqq.

${ }^{5} \mathrm{Da}$ "im Extremfall so viele Derivateme wie derivierende Einheiten gegeben sind", bemerken JACHNOW et al. 1980: 43.

${ }^{6}$ Eine gute Illustration dieser These liefern a) für Werkzeuge und dgl. die Definitionsversuche von Grochowski, von denen stellvertretend GROCHOWSKI 1973a und GROCHOWSKI 1973b genannt seien und b) für Räumlichkeiten und dgl. die Arbeiten von VolOCKAJA 1975 und VILAROVA 1995. 
Mit der Bestimmung der Wortbildungskategorien wird zugleich die Grenze zwischen der Wortbildungsbedeutung und den idiomatischen Bedeutungskomponenten im abgeleiteten Wort gezogen: faßt man die Wortbildungskategorien sehr allgemein, so erhalten sogar Bedeutungskomponenten wie '(handelnde) Person' oder 'Werkzeug' einen idiomatischen Charakter; bei sehr spezifischen Wortbildungskategorien wird dagegen der Bereich der lexikalischen Idiomatik entsprechend verengt.

Die Tatsache, daß eine Festlegung über den Allgemeinheitsgrad der Wortbildungskategorien im Rahmen der syntagmatischen Methode nur apriorisch erfolgen kann, kommt auf die eine oder die andere Weise in sehr vielen Wortbildungsuntersuchungen zum Vorschein. Man nimmt dann die "Diskrepanz zwischen Sprachrealität und ihrer methodischen Erfassungsmöglichkeit" (JACHNOW et al. 1980: 43) resigniert zur Kenntnis und bemüht sich allenfalls, bei der Bestimmung der Wortbildungskategorien einen mittleren Weg einzuschlagen: weder zu allgemein noch zu spezifisch. LENNGREN 1978: 25 hat diesen Grundsatz unverblümt zum Ausdruck gebracht:

Pri sozdanii sistemy semantičeskix slovoobrazovatel'nyx otnošenij glavnaja problema zaključaetsja $v$ tom, čtoby postroit' takuju semantičeskuju set', kotoraja by ne byla sliškom gustoj -- $v$ takom slučae čislo tipov otnošenija [sic!] vozroslo by črezmemo -- $i$ odnovremenno ne propuskala $i$ obobščala by suščestvennye različija.

Eine ähnliche Argumentation findet man bei BuZÁssyovÁ 1974: 52. Je nach Forschertemperament gelangt mán auf diese Weise zu einer geringen Anzahl äußerst abstrakter (DOROSEVSKIJ 1962, LENNGREN 1978 und -- ganz extrem -- LÖNNGREN 1981b) oder zu einer Vielzahl mehr oder weniger konkreter Wortbildungskategorien (so JACHNOw et al. 1980 und bis zu einem gewissen Grade auch SCHUPBACH 1975). (Man stelle sich im Vergleich dazu eine Darstellung des russischen Lautsystems vor, deren Autor im Vorwort erklärt, er wolle nur so viele phonetische Merkmale berücksichtigen, als erforderlich sind, um zu einer überschaubaren Anzahl von Phonemen zu gelangen).

Am häufigsten wird das Problem jedoch dadurch gelöst, daß man die Wortbildungskategorien einfach aus anderen Sprachebenen importiert. (In der Phonologie würde diese Lösung bedeuten, daß man etwa im Russischen unter Verweis auf die Relevanz der Stimmtons bei den Geräuschlauten auch im Bereich der Sonorlaute stimmhafte und stimmlose Phoneme voneinander unterschiede). Wir geben im folgenden einige Beispiele für diese Haltung, wobei wir uns auf slavistische Arbeiten beschränken. So schreibt etwa GLOVINSKAJA 1975: 35:

Vopros svoditsja k tomu, gde vvesti granicu meždu slovoobrazovatel'nym i leksičeskim značeniem. Estestvenno sčitat' slovoobrazovatel'nymi (ne leksiče- 
skimi!) kak minimum takie značenija, kotorye vyražajutsja $v$ jazyke drugimi grammatičeskimi sredstvami -- slovoizmenitel'nymi ili sintaksičeskimi.

Die Aufzählung der Bedeutungen des Suffixes $-(n) i k$, die GlovinSKAJA auf diese Bemerkung folgen läßt, ist vorwiegend an der Syntax orientiert: sub-ekt dejstvija, sostojantja, svojstva, nazvannogo osnovoj; ob-ekt dejstvija, nazvannogo osnovoj; mesto dejstvija, nazvannogo osnovoj; etc. Tatsächlich ist es die Syntax, die (vor allem in der Version der FILLMOREschen Kasusgrammatik) immer wieder der Wortbildung ihre Kategorien spenden muß; die entsprechenden Arbeiten sind äußerst zahlreich und brauchen hier nicht im einzelnen aufgeführt zu werden. Es liegt auf der Hand, daß auch durch den Rückgriff auf die Kasusgrammatik der Apriorismus bei der Bestimmung der Wortbildungskategorien nicht wirklich überwunden wird, da die Relevanz der syntaktischen Unterscheidungen für das Wortbildungssystem keineswegs gesichert ist -- ganz abgesehen von der Frage, daß ja auch im Bereich der Kasusgrammatik über das Inventar der thematischen Rollen (Tiefenkasus, semantischen Rollen, Kasusrollen) keine Übereinstimmung besteht und ihre Anzahl (um nur im Bereich der Russistik zu bleiben) zwischen 3 (so im SOL-System von LENNGREN 1981b) und 25 (APRESJAN 1995, Bd. 1, 125sq.) variiert.?

Neben der Syntax muß auch die Flexionsmorphologie oder die Wortartenlehre als Kategorienspender für die Wortbildung herhalten. So kommt REvZINA 1969: 8sq. durch die Kombination der Beseeltheits- mit der Numeruskategorie zu drei Wortbildungsbedeutungen (agens, actio, res), auf die alle substantivischen Ableitungen zu verteilen seien. Sie faßt dieses Vorgehen mit folgenden Worten zusammen:

Iz skazannogo sleduet, čto gruppy suffiksov, imejuščie obščee slovoobrazovatel'noe značenie, možno vydelit' čisto operacionno, pol'zujas' edinstvom obobščenno-grammatičeskoj semantiki, kotoraja v nix soderžitsja (ibid. p. 9).

SOBOLEVA 1980: 17sq. legt ihrer Klassifikation die Wortartzugehörigkeit des ableitenden und des abgeleiteten Wortes zugrunde. Sie geht von den vier Grundwortarten (Substantiv, Verb, Adjektiv und Adverb) aus, denen sie jeweils eine bestimmte kategoriale Bedeutung (Substanz, Prozeß etc.) zuweist (zu der sogenannten kategorialen Hypothese in der Wortartenlehre cf. LÖNNGREN 1981a). Dadurch ergeben sich insgesamt 16 obščie slovoobrazovatel'nye značenija (41): alle Substantive, die von Verben abgeleitet sind, haben die Wortbildungsbedeutung 'Substanz, die zu einem Prozeß in Beziehung steht' gemeinsam; alle Substantive, die von Adjektiven abgeleitet sind, die Wortbildungsbedeutung 'Substanz, die zu einem Merkmal in Beziehung

${ }^{7}$ Eine Trennlinie zwischen den Kategorien der Syntax und der Wortbildung versucht auch DOKULIL in einem vielzitierten Aufsatz von 1964 zu ziehen; er denkt dabei allerdings in erster Linie an formalsyntaktische Kategorien wie Objekt, Subjekt etc. 
steht'; etc. Was die Termini Substanz oder Merkmal in diesen Umschreibungen bedeuten, bleibt freilich unklar: man beachte, daß etwa die Umschreibung 'Substanz, die zu einem Prozeß in Beziehung steht' sowohl auf Personen- als auch auf Tätigkeitsbezeichnungen Anwendung findet, d.h. nicht nur nomina agentis wie prepodavatel und čitatel', sondern auch nomina actionis wie prepodavanie und ctenie als 'Substanzen' zu gelten haben.

Wie man sieht, führen syntaktische und morphologische Kriterien zu sehr abstrakten Wortbildungsbedeutungen. Die genannten Autoren fühlen sich deshalb immer wieder versucht, durch die Einschaltung einer zusätzlichen Beschreibungsebene zwischen Wortbildungslehre und Lexikologie zu vermitteln: bei REVZINA 1969: 40sqq. ist dies die Ebene der semantičeskaja sxema proizvodnogo imeni, die durch geeignete Paraphrasen (perevodnye ékvivalenty) ermittelt wird, bei SOBOLEVA 1980: 42sqq. die der častnye slovoobrazovatel'nye značenija (SOBOLEVA geht sogar so weit, diese Unterbedeutungen für sprachliche Universalien zu halten).

Eine letzte Möglichkeit besteht schließlich darin, auf eine sprachimmanente Ver- ankerung der Wortbildungskategorien ganz zu verzichten. Eine theoretische Begründung findet dieses Vorgehen in der Behauptung, sprachliche Kategorien seien bereits durch die fundamentalen Kategorien der menschlichen Kognition vorgegeben. Für die morphologische Wortbildung wurde diese Annahme von BOGDAN SZYMANEK zu einem methodischen Forschungspostulat erhoben:

\section{The Cognitive Grounding Condition}

The basic set of lexical derivational categories is rooted in the fundamental concepts of cognition.

(SzYMANEK 1988: 93; cf. die Rezeption bei BEARD 1995: 208sqq., der von "Cognitive Grounding Principle" spricht).

Der Gebrauch der Termini "condition" bzw. "principle" zeigt, daß die kognitive Verankerung der Wortbildungskategorien nicht das Ergebnis empirischer Forschung, sondern eine apriorische Annahme (und vielleicht sogar eine leere Tautologie) darstellt. Will man die "Cognitive Grounding Condition" in eine empirisch überprüfbare Hypothese zurückverwandeln, so muß ein Verfahren entworfen werden, das es erlaubt, sprachliche Kategorien nach rein sprachimmanenten Kriterien, d.h. ohne Rückgriff auf die kognitive Psychologie zu bestimmen.

All diese Versuche sind nur ein Zeichen dafür, daß der Apriorismus bei der Bestimmung der Wortbildungskategorien im Rahmen der syntagmatischen Methode nur überspielt, aber nicht wirklich beseitigt werden kann: die Grundeinheiten, mit denen hier operiert wird, werden nicht aus der Wortbildungslehre abgeleitet, sondern in sie hineingetragen (cf. DEBATY-LUCA 1986: 142-152). 
Diesem grundlegenden Mangel der syntagmatischen Methode sucht nun die paradigmatische Methode der Wortbildungslehre dadurch abzuhelfen, daß sie nicht das Wortbildungspaar, sondern das Wortbildungsparadigma zum Ausgangspunkt der Wortbildungsanalyse macht. Zum Wortbildungsparadigma eines ableitenden Wortes müssen all jene Wörter gezählt werden, die von ihm direkt (d.h. ohne die Zwischenstufe eines weiteren abgeleiteten Wortes) abgeleitet sind. So gehören etwa zum Wortbildungsparadigma von kurit' u.a. folgende Ableitungen:

kuril'šcik
kurevo
kurilka
kuritel'nyj
zakurit'
perekurit'
trubokur

(Die genaue Definition des Wortbildungsparadigmas wird in Kapitel 2 gegeben; dort gehen wir auch auf eine Reihe von Einzelproblemen ein: die Zulassung von Beziehungsadjektiven als Zwischenstufe und die Behandlung zusammengesetzter Wörter.)

Der Grundgedanke der paradigmatischen Methode besteht nun darin, die Wortbildungskategorien nicht willkürlich festzulegen, sondern aus den Wortbildungsparadigmen einer Sprache durch funktionale Oppositionen auszugliedern; dabei kommt dem Prinzip der Relevanz (frz. pertinence) zentrale Bedeutung zu. Es ist das Verdienst von THIERRY DEBATY-LUCA, diesem Prinzip ein beredtes (wenn auch gelegentlich etwas redseliges) Plädoyer gewidmet zu haben (DEBATY-LUCA 1986, cf. die zusammenfassende Darstellung DEBATY-LuCA 1985). Er hat ihm auf p. 160 seiner Abhandlung folgende Formulierung gegeben:

Le principe qui régira toute lanalyse est donc le suivant: une distinction sur le plan de lexpression sera considérée comme pertinente si elle saccompagne dune distinction sur le plan du contenu et vice versa.

Wir wollen nun an einigen Beispielen zeigen, wie der Allgemeinheitsgrad von Wortbildungskategorien (und damit auch die Grenze zwischen Wortbildungsbedeutung und idiomatischer Bedeutung im abgeleiteten Wort) im Rahmen der paradigmatischen Methode der Wortbildungsanalyse mit Hilfe des Prinzips der Relevanz bestimmt werden kann. Man sieht sofort, daß die Anwendung dieses Prinzips die Zusammenfassung der oben angeführten Bezeichnungen für handelnde Personen, Werkzeuge und Räumlichkeiten (mečtatel', gasitel, vytrezvitel) zu einer Wortbildungskategorie verbietet: in zahlreichen deverbalen Wortbildungsparadigmen des Russischen wird nämlich die semantische Opposition zwischen dem Vollstrecker der Verbalhandlung, dem Werkzeug, das zu ihrer Ausführung dient, und dem Or, der 
dafür vorgesehen ist, durch formal unterschiedliche Ableitungen zum Ausdruck gebracht:
prjadil'nja 'Spinnerei, Spinnfabrik'
prjalka 'Spinnrad, Trittrad'
prjadil'ščik 'Spinner'
plavil'nja 'Schmelzhütte, Schmelze'
plavil'nik 'Schmelztiegel'
plavil'ščik 'Schmelzer'

Auch die Bedeutungskomponente 'groß' in glazastyj erscheint in einem anderen Licht, wenn man sich die Frage stellt, ob es im Russischen desubstantivische Wortbildungsparadigmen gibt, in denen ihr Fehlen bzw. Vorhandensein durch zwei formal unterschiedliche Ableitungen zum Ausdruck gebracht wird. Solche Wortbildungsparadigmen lassen sich tatsächlich unschwer ausfindig machen:

usatyj 's usami ili s bol'šimi usami' 'schnurrbärtig; mit großem Schnurrbart' usastyj 's bol'šimi usami' 'mit großem Schnurrbart'

rogatyj 's rogami ili s bol'šimi rogami' 'behörnt; mit großen Hörnern' rogastyj 's bol'šimi rogami' 'mit großen Hörnern'8

Die Bedeutungskomponente 'groß' ist nach Ausweis von SVE im jeweils ersten Adjektiv fakultativ, im zweiten dagegen obligatorisch; andere Wörterbücher lassen die Bedeutungskomponente 'groß' bei den Adjektiven auf -atyj ganz aus, cf. etwa die Erklärung von rogatyj im MAS: 'imejuščij roga'. Zwischen den Adjektiven auf -atyj und den Adjektiven auf -astyj besteht überdies ein deutlicher stilistischer Unterschied, da die letzteren dem prostorečie näher stehen. Vielleicht ist der Stilwert als das primäre Unterscheidungsmerkmal anzusehen, während die Bedeutungskomponente 'groß' eine Begleiterscheinung darstellt, die durch die Neigung der volkstümlichen Ausdrucksweise zum Hyperbolismus zu erklären wäre; cf. den leicht pejorativen Charakter von borodastyj, usastyj etc., der auch den Gebrauch des Suffixes -astyj bei bel'mastyj zu erklären hilft. Eines machen die zitierten Oppositionen jedoch klar: man kommt in diesem Bereich der desubstantivischen Wortbildung des Russischen nicht

${ }^{8}$ Cf. EFremova 1996: 62 und 64, die als Kontrastpaar nosatyj :: nosastyj nennt. $\mathrm{Zu}$ den Adjektiven auf -atyj und -astyj s. außerdem DANILENKO 1954 und MALKIEL 1978: 137, der treffend bemerkt, die Adjektive auf -astyj "tend to draw a caricature rather than a portrait of the human being involved", und Parallelen aus dem Spanischen anführt. 
mit einer einzigen Wortbildungskategorie aus, sondern braucht zur Beschreibung der entsprechenden Ableitungen (mindestens) zwei; die Bedeutungskomponenten, die usastyj von usatyj, rogastyj von rogatyj etc. unterscheiden, gehören -- mögen sie nur denota-tiven oder mehr stilistisch-expressiven Charakter haben -- der Wortbildungsbedeutung dieser Adjektive an.

Als letztes Beispiel wählen wir die nomina loci (Orts- und Räumlichkeitsbezeichnungen). Z.M. VOLOCKAJA hat diesen Ableitungen 1975 eine Untersuchung gewidmet, die sich für eine Gegenüberstellung der paradigmatischen und der syntagmatischen Methode der Wortbildung besonders gut eignet. ${ }^{9}$ Die gemeinsame Wortbildungsbedeutung der nomina loci gibt VolockAJA mit 'tam, gde' an, z.B.

$$
\begin{aligned}
& \text { kupal'nja = 'tam, gde kupajutsja' } \\
& \text { lesopil'nja = 'tam, gde piljat les' } \\
& \text { pożarišče = 'tam, gde byl požar'. }
\end{aligned}
$$

Da sich die derart definierte Wortbildungskategorie jedoch als zu allgemein erweist, wird sie in eine Reihe von Unterkategorien aufgespalten, die als Derivationsklassen bezeichnet werden; im Bereich der desubstantivischen Wortbildung sind dies etwa:

a) denominale nomina loci, bei denen das ableitende Substantiv agentivischen Charakter hat und das abgeleitete den Ort bezeichnet, an dem die entsprechende Tätigkeit (arbeiten etc.) ausgeführt wird: parikmaxerskaja 'Frisiersalon', detskaja 'Kinderzimmer', slesarnja 'Schlosserwerkstatt', pekarnja 'Bäckerei', kočegarka 'Heizraum einer Dampfkesselanlage', storožka 'Wächterhäuschen', kuznica 'Schmiede' (42sq.)

b) denominale nomina loci, bei denen das Basissubstantiv den Gegenstand bezeichnet, der produziert oder bearbeitet wird: lesopil'nja '(kleines) Sägewerk', degtjarnja 'Teerbrennerei', solodovnja 'Malztenne, Mälzerei' (43sq.)

c) denominale nomina loci, bei denen das Basissubstantiv den Gegenstand bezeichnet, der irgendwo aufbewahrt oder gelagert wird; als Subtyp treten hier Bezeichnungen für Läden auf, in denen der betreffende Gegenstand verkauft wird: moločnaja 'Milchhandlung', buločnaja 'Bäckerladen', pirožkovaja 'Imbißstube, in der Piroggen [gebacken und] verkauft werden', žitnica 'Kornkammer', kartofelexranilišče 'Kartoffelmiete', kofejnja 'Kaffeehaus' (44sq.)

9 Die (ungedruckte) Abhandlung von RENATE BELENTSCHIKOW, Untersuchungen zu den Nomina loci in der russischen Sprache der Gegenwart. -- Berlin, Humboldt-Univ., Diss. A 1982 war mir leider nicht zugänglich. Zu den nomina loci im Slavischen cf. BEARD 1995: 87sqq., 318sqq. Eine Auseinandersetzung mit den anregenden Thesen von R. BEARD würde den Rahmen dieser Einleitung sprengen. 
d) denominale nomina loci, bei denen das Basissubstantiv ein Lebewesen ist und die Ableitung seinen Lebensraum bezeichnet: korovnik 'Kuhstall', golubjatnik 'Taubenschlag', medveżatnik 'Bärenkäfig', slonovnik 'Elefantenkäfig, Elefantengehege', skvorečnja 'Starkasten', ovčarnja 'Schafstall', volovnja 'Ochsenstall', zverinec 'Tierschau in engen Käfigen' (45sq.)

e) denominale nomina loci, bei denen das Grundwort eine Pflanze bezeichnet und die Ableitung den Ort, wo diese Pflanze wächst: el'nik 'Fichtenwald', kedrovnik 'Zedernwald', pixtarnik 'Edeltannenwald', ol'šannik 'Erlenwald', sosnjak 'Kiefernwald', ivnjak 'Weidengehölz', kleverišče 'Kleewiese', goroxovišče '(gewesenes) Erbsenfeld' (49sq.)

Es liegt auf der Hand, daß damit die Differenzierungsmöglichkeiten noch keineswegs erschöpft sind; wir wollen das an der letzten Derivationsklasse illustrieren, der u.a. folgende Ableitungen zuzurechnen sind:

risovišče 'pole posle uborki risa' (NS) 'abgeerntetes Reisfeld' grušovnik 'gruševyj sad' (BASl) 'Birnengarten'

limonarij 'pitomnik, v kotorom vyraščivajut limony' (MAS) 'Pflanzstätte für Zitronenbäume'

Nichts spricht im Rahmen der syntagmatischen Methode dagegen, die Bedeutungskomponenten '(abgeerntetes) Feld', 'Garten' und 'Pflanzstätte', denen überdies unterschiedliche Wortbildungsformanten entsprechen, zu Bestandteilen der Wortbildungsbedeutung zu erklären und die entsprechende Derivationsklasse in drei Unterklassen aufzuteilen. Vom Standpunkt der paradigmatischen Wortbildungslehre dürften dagegen bereits die Derivationsklassen a) bis e) zu spezifisch sein: hier kommt alles auf das Vorhandensein von Wortbildungsparadigmen an, in denen mehrere nomina loci zueinander in formaler und inhaltlicher Opposition stehen. Es dürfte schwer sein, auf diese Weise die Relevanz der Oppositionen nachzuweisen, die mit den Derivationsklassen von VolOCKAJA gebildet werden können (von der Opposition '(abgeerntetes) Feld :: Garten :: Pflanzstätte' ganz zu schweigen): Bedeutungskomponenten wie 'arbeiten', 'wachsen' oder 'hergestellt werden' sind ja durch die Semantik des ableitendes Wortes vorgegeben und deshalb nicht substitutionsfähig.

Es ist allerdings nicht so, als ob die Wortbildungskategorie der nomina loci im Rahmen der paradigmatischen Methode keiner weiteren Differenzierung fähig wäre. In einer Reihe von desubstantivischen Wortbildungsparadigmen treten tatsächlich zwei formal und inhaltlich unterschiedliche nomina loci auf, von denen das eine eine Produktionsstätte, das andere einen Lagerraum bezeichnet:

degtjarnica 'Teerbüchse' (PAW) 'sosud (bočka, vedro, banka i t.p.) dlja degtja' (BAS2) vs. 
degtjarnja 'Teerbrennerei, Teersiederei' (PAW) 'mesto, pomeščenie, gde osuščestvljaetsja vygonka degtja ili propityvanie čego-l. degtem' (BAS2)

syrnica 'Käseglocke', 'stolovaja posuda dlja syra' (NS-2) vs.

syrovarnja 'Käserei' (PAW), 'predprijatie, izgotovljajuščee syr' (MAS), cf. auch das veraltete syrnja 'Käsehaus, Käsekammer, Käserei' (PAW)

xlebnica 'Brotkorb' (PAW) 'tarelka ili korzinka dlja xleba' (BAS1) vs. xlebnja 'Bäckerei, Brotbäckerei; Brotladen, -bude' (PAW) 'ustar. pomeščenie dlja vypečki xleba; pekarnja' (BASl)

Diese und ähnliche Oppositionen zwingen im Rahmen der paradigmatischen Wortbildung, im Bereich der desubstantivischen nomina loci von (mindestens) zwei Wortbildungskategorien auszugehen und die Bedeutungskomponente 'Produktion' sowie 'Lagerung' in der Inhaltsseite der entsprechenden Ableitungen der Wortbildungsbedeutung zuzurechnen (cf. die Gegenüberstellung von "dinamična" und "statična xarakteristika" bei VILAROVA 1995: 8).

Der Begriff des Wortbildungsparadigmas ist im Laufe der letzten Jahrzehnte immer stärker ins Zentrum der slavistischen Wortbildungsforschung gerückt; sein logisches Korrelat, das Prinzip der Relevanz, wird allerdings nur selten in seiner ganzen Tragweite erkannt. Wir wollen dies an einigen ausgewählten Wortbildungsarbeiten aus dem erwähnten Zeitraum illustrieren, die wir in der Reihenfolge ihres Erscheinens besprechen:

Bei RevZINA/REvZIN 1967: 1658sqq. ist die Unterscheidung des syntagmatischen und des paradigmatischen Zugangs zur Wortbildung bereits getroffen; letzterer liege immer dann vor, wenn man von nomina actionis, nomina agentis, nomina situativa etc. spreche. Diese Wortbildungskategorien, deren Gültigkeit für alle slavischen Sprachen (a priori?) feststehe, werden in der Nachfolge DokUliLs als onomasiologisch bezeichnet und einer Reihe von qualifikativen Merkmalen wie Intensität, Terminativität und Subjektivität gegenübergestellt. Während die onomasiologischen Wortbildungskategorien gleichsam durch die Struktur der Wirklichkeit selbst vorgegeben seien, komme in den qualifikativen Merkmalen die subjektive Seite des Wortbildungsprozesses zum Ausdruck: sie beträfen weniger den benannten Gegenstand selbst als den Gesichtspunkt, unter dem der Sprecher den Nominationsakt vollziehe. Als diagnostisches Mittel werden funktionale Oppositionen deshalb lediglich zur Ermittlung der qualitativen Merkmale herangezogen -- und auch dies nur am Rande (1660sq.). Diese Tendenz setzt sich in REvzinA 1969 fort, wo auf p. 25 in Bezug auf das qualitative Merkmal der Intensität folgendes Prinzip der neizbytočnost (Ökonomie) aufgestellt wird: 
Étot princip sostoit $v$ tom, čto esli ot kakoj-to osnovy imeetsja nejtral'noe obrazovanie s suffiksom dannogo polja, $v$ nem, kak pravilo, otsutstvuet proizvodnoe $s$ usilitel'nym suffiksom togo że polja.

Die Gültigkeit dieses Prinzips wäre im Rahmen der paradigmatischen Methode gerade ein Argument dafür, den Gegensatz zwischen intensivierenden und neutralen Suffixen im Bereich der nomina agentis als wortbildungsirrelevant einzustufen.

Auch in der Monographie von REvzina 1969 bildet die Gegenüberstellung von "deskriptiver" ("syntagmatischer") und "paradigmatischer" Wortbildungslehre den Ausgangspunkt (p. 4); als grundlegende Einheit des Wortbildungssystems sieht die Autorin das "Wortbildungsfeld" (slovoobrazovatel'noe pole) an, dessen Mitglieder durch eine gemeinsame, äußerst abstrakte Wortbildungsbedeutung zusammengehalten werden. Es wurde oben bereits bemerkt, daß die Wortbildungsfelder unter Rückgriff auf die Flexionsmorphologie bestimmt werden: die Beseeltheitskategorie grenzt die Ableitungen des slovoobrazovatel'noe pole dejatelja (also die nomina agentis; richtiger wäre freilich: nomina agentis oder patientis) von den Ableitungen der beiden übrigen Wortbildungsfelder $a b$; die Numeruskategorie unterscheidet wiederum die Ableitungen des slovoobrazovatel'noe pole dejstvija $i$ sostojanija, die in der Regel keine Pluralformen besitzen, von den Ableitungen des slovoobrazovatel'noe pole vešci. Immerhin sind hiermit semantische Oppositionen benannt, die zumindest in einem Teil der Flexionsformen der betreffenden Ableitungen eine formale Entsprechung besitzen; es ist jedoch nicht einzusehen, warum die viel auffälligeren formalen Oppositionen, mit deren Hilfe die drei erwähnten Wortbildungsfelder weiter untergliedert werden können, keine eigenen Wortbildungskategorien (oder "Wortbildungsfelder") konstituieren sollten. Die Diskussion des Problems der idiomatischen Bedeutungskomponenten im abgeleiteten Wort auf p. 48sqq. zeigt deutlich, daß Revzina trotz ihres paradigmatischen Ansatzes das Prinzip der Relevanz letztlich doch nicht anzuwenden weiß.

DúROVIĆ 1972: 196 postuliert für das Wortbildungssystem des Russischen die Existenz von "Mikrostrukturen (Paradigmen) mit einer festen Anzahl von Elementen (Positionen) und einem vorgegebenen Inventar von semantischen distinktiven Merkmalen. In einzelnen Fällen können bestimmte Positionen nicht realisiert bleiben, aber die Existenz von Wörtern, deren Bedeutung durch die Mikrostruktur nicht vorgegeben wäre, ist unmöglich" (meine Übersetzung). Das Prinzip der Relevanz wird nirgends ausdrücklich erwähnt, darf aber vielleicht vorausgesetzt werden, wenn die Rede von distinktiven Merkmalen ist. Leider hat DuROVIĆ die Existenz solcher Paradigmen mehr postuliert als tatsächlich aus dem Material abgeleitet; jedenfalls wird in seiner Arbeit nicht das gesamte Wortbildungsparadigma der Beispielsubstantive člen, aspirant, vuz, komsomol berücksichtigt, sondern nur der Ausschnitt, der das Beziehungsadjektiv, die Motion und das Kollektivum betrifft. 
Dieser Vorwurf trifft die bekannte Monographie von KLÁRA BUZÁSSYOVÁ über das slovakische Deverbativum nicht: in dieser Arbeit, die 1974 erschienen ist, wird eine Vielzahl von deverbalen Wortbildungsparadigmen analysiert; jede Ableitung wird einer bestimmten Wortbildungskategorie zugewiesen. Bei der semantischen Analyse dieser Wortbildungskategorien unterscheidet die Autorin zwei Arten von Merkmalen: die primären (kategorialen) und die sekundären (Begleit)merkmale. Die Grenze zwischen Wortbildung und Lexikologie wird p. $41(\S 3,60)$ "deduktiv" dadurch bestimmt, daß alles, was nach der Aufzählung der kategorialen und sekundären Merkmale einer Ableitung übrigbleibt, zur lexikalischen (idiomatischen) Bedeutung des entsprechenden Wortes gehört. Hinter den kategorialen Merkmalen, die auf p. 56 $(\S 5,21)$ aufgezählt werden, verbergen sich die bekannten, an der Verbalsyntax orientierten Wortbildungskategorien DoKULILscher Provenienz (nomina agentis, nomina instrumenti, nomina actionis etc.); die Begleitmerkmale (Beseeltheit, Konkretheit, Kollektivität, Intensität etc.) erinnern an die qualifikativen Merkmale RevZINAS. BUZÁSSYOVÁ folgt REvZINA auch darin, daß sie funktionale Oppositionen als diagnostisches Instrument nahezu ausschließlich bei den Begleitmerkmalen einsetzt; die primären, kategorialen Merkmale scheinen ihre Gültigkeit unabhängig davon zu besitzen, ob sich mit ihnen innerhalb ein und desselben Wortbildungsparadigmas Oppositionen bilden lassen oder nicht (p. 68, § 5,40; cf. p. 57sq., $\S 5,21$ zur Begründung der Einführung getrennter kategorialer Merkmale für Instrument und Material, Resultat und Überbleibsel). In diesem Zusammenhang verwundert es nicht, wenn die Autorin bei der Bestimmung der Wortbildungskategorien auch dem rein praktischen Kriterium der Übersichtlichkeit der Klassifikation eine gewisse Gültigkeit einräumt: sowohl die kategorialen als auch die sekundären Merkmale müssen so allgemein wie möglich sein, damit eine zu große Detailliertheit der Wortbildungsbedeutungen vermieden wird (p. 52, 5 5,01).

Auch bei SCHUPBACH 1975, der doch den paradigmatischen Aspekt der Worthildung in den Vordergrund der Betrachtung rücken will, sucht man vergeblich nach dem Kriterium der semantischen Relevanz von formalen Oppositionen innerhalb des Wortbildungsparadigmas; die Einführung der einzelnen Wortbildungskategorien (derivative types) orientiert sich in erster Linie an deren Fähigkeit, (weitere) Ableitungen zu bilden (p. 42sq., cf. p. 53sq. und p. 135sq.). Das Ergebnis ist eine recht heterogene Liste von derivative types, in der neben solchen klassischen Wortbildungskategorien wie expressive substantives oder substantives meaning "object" or "place" auch für die rein formal definierte Kategorie der Substantive auf -stv(o) sowie die lexikalische Gruppe der generic terms for plants and animals Platz ist.

Auf den Begriff des Wortbildungsparadigmas ist auch El.ENA ANDREEVNA ZEMSKAJA mehrfach eingegangen. In dem bekannten Aufsatz von 1978 (ZEMSKAJA 1978) wird festgestellt, daß die "Wortbildungskategorien, ähnlich wie die grammatischen Kategorien, einander nach Oppositionen gegenübergestellt werden können" (ZEMSKAJA 1978: 69). Diese Formulierung wird der konstitutiven Rolle, die formal ausgedrückten 
Bedeutungsoppositionen im Wortbildungsparadigma zukommt, nicht ganz gerecht; offen bleibt auch, ob die bereits auf anderem Wege ermittelten Wortbildungskategorien oder aber Mitglieder eines konkreten Wortbildungsparadigmas, denen diese Kategorien zugrundeliegen, einander gegenübergestellt werden sollen. So ist etwa, um ein von ZEMSKAJA (p. 70) nach R.S. MANUĊARJAN zitiertes und auch in dieser Einleitung behandeltes Beispiel aufzugreifen, die Gegenüberstellung von krylatyj und nosatyj nicht ausreichend, um die Relevanz des Merkmals [ \pm intensiv] im Bereich der desubstantivischen Wortbildung zu begründen, da ja diese Ableitungen verschiedenen Wortbildungsparadigmen angehören.

Im Vergleich zu ZEMSKAJA 1978 stellt die Diskussion des Begriffes Wortbildungsbedeutung (slovoobrazovatel'noe značenie) bei ZEMSKAJA 1992 einen gewissen Rückschritt dar. Es wird zwar eingeräumt, daß der Abstraktionsgrad von Wortbildungsbedeutungen nicht a priori festgesetzt werden kann, sondern von der Struktur des untersuchten Wortschatzausschnittes (Personen-, Handlungs- und Sachbezeichnungen) abhängt (33sq.); doch ein Verfahren, mit dem man diese Unterschiede aufzeigen kann, wird nicht genannt. Daß die Zusammenfassung von Ableitungen nach ihrer formalen und inhaltlichen Ähnlichkeit (p. 28, Punkt 2) nicht genügt, liegt auf der Hand. Bei der Diskussion des Status der Bedeutungskomponenten 'Fleisch von...' in Ableitungen wie konina, baranina bringt ZEMSKAJA ein weiteres Argument ins Spiel: die Komponente 'Fleisch von...' gehe trotz ihres konkreten Charakters in die Wortbildungsbedeutung der betreffenden Ableitungen ein, da sie über ein spezifisches Wortbildungsmittel [das Suffix -'atin(a)] verfüge. ${ }^{10}$ Dieses Kriterium kann offensichtlich keine allgemeine Gültigkeit beanspruchen: die bekannte Erscheinung der Affixhomonymie (oder -polysemie) bringt es ja mit sich, daß eine ganze Reihe traditioneller Wortbildungskategorien über keine spezialisierten Wortbildungsmittel verfügen (für den Bereich der Sachbezeichnungen des Russischen hat dies REVZINA 1969 ermittelt).

Einen anregenden Beitrag zur Entwicklung der paradigmatischen Wortbildungsmethode stellt die Monographie von THIERRY DEBATY-LUCA dar, der wir bereits die Formulierung des Prinzips der Relevanz entnommen haben: die Théorie fonctionnelle de la suffixation, die 1986 in Buchform erschienen ist (DEBATY-LUCA 1985, 1986). Der Autor dieser Arbeit, in der die paradigmatische Methode der Wortbildungslehre in ihrer reinsten Form vertreten ist, lehnt sich an den Funktionalismus von ANDRE MARTINET an; neuere (generative oder kognitive) Forschungsansätze werden beiseite-

10 Dieses Suffix ist eine Erweiterung von -in(a), womit nicht nur Fleischbezeichnungen, sondern auch Singulativa und Augmentativa gebildet werden. $\mathrm{Zu}$ der allmählichen Differenzierung dieser drei Kategorien durch die Weiterentwicklung von -in(a) zu -ink(a) und -atin(a) s. ZEMSKAJA 1975b. Es gibt aber immer noch Ableitungen auf - $\operatorname{atin}(a)$, die anderen semantischen Gruppen angehören, cf. osljatina 'prostoreč. o tupom i uprjamom čeloveke' (BASI). 
gelassen -- von der slavistischen Wortbildungslehre ganz zu schweigen. Diese Einseitigkeit hat -- zusammen mit vielen unnötigen terminologischen Neubildungen und einer ermüdenden Weitschweifigkeit -- dem durchaus bedenkenswerten Ansatz von DEBATY-LUCA die Beachtung versagt, die er eigentlich verdient hätte (cf. die negative Rezension von WANDRUSZKA 1989).

Fast gleichzeitig mit DEBATY-LUCA hat der niederländische Forscher JAAP VAN MARLE eine umfangreiche Monographie über die paradigmatische Dimension der morphologischen Kreativität veröffentlicht (VAN MARLE 1985). VAN MARLE untersucht die Faktoren, die die Verteilung konkurrierender Affixe sowohl in der Formenals auch in der Wortbildung des Niederländischen steuern, und gelangt dabei zur Formulierung einer Hypothese, der "domain hypothesis", die er allerdings mehr als heuristisches Prinzip denn als gültige Generalisierung verstanden wissen will (VAN MARLE 1985: 221-241, cf. VAN MARLE 1986). Es liegt auf der Hand, daß dieser Fragestellung ein abweichendes Verständnis von Paradigmatik zugrundeliegt: konkurrierende Affixe stehen zueinander in komplementärer Distribution und können deshalb einer Analyse nach funktionalen Oppositionen nicht unterzogen werden. Die Probleme, die uns in dieser Einleitung beschäftigen, werden bei VAN MARLEs Ansatz bereits als gelöst angesehen: die Feststellung, daß zwei oder mehrere Affixe bzw. Wortbildungstypen (von VAN MARLE als morphological categories bezeichnet) innerhalb einer Wortbildungskategorie zueinander in Konkurrenz stehen, setzt ja die Aufstellung von Wortbildungskategorien und damit eine Wortbildungsanalyse (sei sie nun syntagmatischer oder paradigmatischer Art) voraus. Neben dieser zentralen Fragestellung finden bei VAN MARLE auch Substitutionsbeziehungen zwischen den Affixen Berücksichtigung (VAN MARLE 1985: 85-87, 154sq., 258sqq.).

Die Gemeinsamkeiten und die Unterschiede zwischen den Flexions- und den Wortbildungsparadigmen sind schon mehrmals behandelt worden (KLOBUKOVA 1981; KUBRJAKOVA/SOBOLEVA 1979; KRASIL'NIKOVA 1989, die gegen den Begriff des Wortbildungsparadigmas polemisiert; JUSUPOVA 1980: 4sq.; BAUER 1997). Einen ausführlichen Vergleich zwischen der Formen- und der Wortbildung hat neuerdings MELČ́UK 1993-94 unternommen, der die Rolle der paradigmatischen Beziehungen (Oppositionen) bei der Aufstellung von Wortbildungskategorien (Derivatemen) in Frage stellt (Bd. 1, p. 288sq.) und den Terminus Paradigma ausschließlich der Formenbildung vorbehalten will (ibid. p. 356, Def. 1.45). Nach der Auffassung von MEL'ĆUK gewinnen die Grundeinheiten der Formenbildung, die Grammeme, nur im Rahmen einer (mindestens zweigliedrigen) grammatischen Kategorie ihre Konturen (cf. die Definitionen des "Grammems" p. 264 [Def. 1.31] und der "Kategorie" p. 261sq. [Def. I.29], bes. die Klarstellung unter Nr. 3 auf p. 262); Derivateme können dagegen auch isoliert auftreten, ohne an Eigenständigkeit einzubüßen. Eine ausführliche Auseinandersetzung mit der morphologischen Konzeption MEL'ĆUKs, in die zwangsläufig auch andere Aspekte seines terminologischen Systems einbezogen werden müßten, würde den Rahmen der vorliegenden Einführung sprengen; es sei 
lediglich angemerkt, daß die Möglichkeit einer Strukturierung von Derivatemen durch paradigmatische Oppositionsreihen auch von MEL ¿̇UK letzten Endes nicht geleugnet werden kann (p. 289). Die Wirksamkeit paradigmatischer Faktoren im Wortbildungsproze $\beta$ tritt in morphologischen Ersetzungsbildungen wie zudecken/aufdecken (VAN MARLE 1985: 258-292; BECKER 1993) und in diachronen Erscheinungen wie der Differenzierung der Suffixe -in(a)/-ink(a)/-atin(a) im Russischen (ZEMSKAJA 1975b) deutlich zutage.

Auf den ersten Blick möchte es nun scheinen, als hätten wir mit der paradigmatischen Methode endlich den archimedischen Punkt gefunden, von dem aus die Wortbildungslehre als eigene linguistische Teildisziplin in klarer Abgrenzung zur Lexikologie aufgebaut werden könnte -- eine Methode, die es erlaubt, die Grundeinheiten der Wortbildung, die Wortbildungskategorien, an deren eigenem Untersuchungsgegenstand abzulesen und nicht von außen an sie heranzutragen; diese These wird ja in der erwähnten Monographie von DEBATY-LUCA aufs vehementeste vertreten. Die Gewißheit, mit der paradigmatischen Methode endlich festen Boden unter den Füßen gefunden zu haben, hält jedoch einer genaueren Betrachtung nicht stand. Auch in diesem Bereich zeigt sich, daß die Sprache ein System ist, zwischen dessen Teileinheiten ein durchgängiger Zusammenhang besteht -- un système, où tout se tient: was man auf der einen Seite einnimmt, muß man auf der anderen Seite sofort wieder ausgeben.

Die Achillesferse der paradigmatischen Methode stellt die Annahme dar, daß den einzelnen, konkreten Wortbildungsparadigmen, von denen sie bei der Analyse ausgeht, ein und dasselbe Koordinatensystem von Oppositionen, d.h. ein und dasselbe abstrakte Wortbildungsparadigma zugrundeliegt. ( $\mathrm{Zu}$ der Unterscheidung von abstrakten und konkreten Wortbildungsparadigmen cf. den bereits besprochenen Aufsatz von ZEMSKAJA 1978: 73sq. sowie ZEMSKAJA 1992: 17-25. ZEMSKAJA gebraucht die Termini konkretnaja bzw. tipovaja slovoobrazovatel'naja paradigma.) Bei unseren Illustrationen der paradigmatischen Methode sind wir ja vorhin stillschweigend davon ausgegangen, daß die Verben mečtat', gasit, vytrezvit', prjast', plavit, ..., die Substantive glaz, rog, usy, ... bzw. degot, xleb, syr, kapusta, kartofel, ... in ihren Ableitungen jeweils dasselbe System von Wortbildungskategorien realisieren -- eine Annahme, die im Rahmen der paradigmatischen Methode mit mehr oder weniger plausiblen Argumenten verteidigt, aber im strengen Sinne des Wortes nicht begründet werden kann: ihre Begründung würde nämlich die Kenntnis gerade jener Wortbildungskategorien voraussetzen, die mit ihrer Hilfe ermittelt werden sollen. Die Frage, welche konkreten Wortbildungsparadigmen miteinander verglichen werden sollen, d.h. die Frage nach dem Geltungsbereich der abstrakten Wortbildungsparadigmen einer Sprache kann die paradigmatische Methode der Wortbildungslehre nur apriorisch entscheiden. Auch hier sind zwei extreme Lösungen denkbar: man kann jedes konkrete Wortbildungsparadigma zur Realisation eines eigenen abstrakten Wortbildungsparadigmas erklären, um auf diese Weise zu Wortbildungskategorien zu 
gelangen, die sehr nah am lexikalischen Material angesiedelt sind (man denke nur an die Opposition montažnik :: montažer :: montažist); oder man kann, umgekehrt, abstrakte Wortbildungsparadigmen von maximalem Umfang bilden und dabei jene funktionalen Oppositionen vernachlässigen, für die sich nur vereinzelte Belege finden lassen: die Wortbildungskategorien werden dadurch abstrakter, die Grenze zwischen der Wortbildungsbedeutung und der idiomatischen Bedeutung des abgeleiteten Wortes wird zugunsten der idiomatischen Bedeutung verschoben. (Die Forderung, eine Opposition müßte in allen konkreten Paradigmen vertreten sein, um als kategorienkonstitutiv zu gelten, kann im Bereich der Wortbildung wegen der relativ großen Variationsbreite der Paradigmen und der häufigen Fälle von Defektivität nicht aufrechterhalten werden.)

Was also für die syntagmatische Methode der Übergang vom Wortbildungspaar zur Wortbildungskategorie ist, das ist für die paradigmatische Methode der Übergang vom konkreten zum abstrakten Wortbildungsparadigma: der Sprung von einem Wortbildungsparadigma zum anderen -- von prjadilnja :: prjalka zu plavil'nja :: plavilnik -- ist genauso problematisch wie der Sprung vom einen Wortbildungspaar

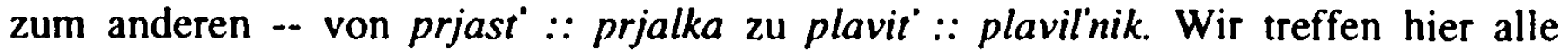
Unsicherheiten wieder, die wir schon bei der Bestimmung der Wortbildungskategorien im Rahmen der syntagmatischen Methode kennengelernt haben. Man kann den Geltungsbereich eines abstrakten Wortbildungsparadigmas nach rein praktischen Gesichtspunkten festlegen: weder zu groß noch zu klein; oder man greift auf andere Sprachbereiche zurück -- die Lexikologie, die Syntax oder auch die Wortartenlehre. Am verbreitetsten ist die Übernahme der grundlegenden Kategorie (des abstrakten Wortbildungsparadigmas) aus der Lexikologie: sie besteht in der Annahme, daß die Mitglieder eines Wortfeldes in ihren Ableitungen dasselbe abstrakte Wortbildungsparadigma realisieren. (Diese Annahme liegt einer Reihe von Dissertationen zugrunde, die unter der Anleitung von E. A. ZEMSKAJA entstanden sind; cf. die Aufzählung bei ZEMSKAJA 1992: 18, Anm. 2.) Aber auch eine Anlehnung an die Syntax ist möglich: man kann z.B. davon ausgehen, daß alle Verben eines bestimmten Valenztyps auch ihre Wortbildungskategorien gemeinsam haben (dieser Lösung nähert sich die bereits mehrfach zitierte Arbeit von BUZÁsSYOVA 1974). Von der Wortartenlehre geht schließlich DEBATY-LUCA 1986 aus, wenn er dafür plädiert, alle Wortbildungsparadigmen der Wörter einer bestimmten Wortart als Realisationen ein und desselben abstrakten Wortbildungsparadigmas ("suffixalen Systems") anzusehen. Solche Entscheidungen sind von Fall zu Fall mehr oder weniger plausibel; sie können jedoch -und das ist das Entscheidende -- mit den Mitteln der paradigmatischen Wortbildungslehre nicht begründet werden: um konkrete Wortbildungsparadigmen nach reinen Wortbildungskriterien zu abstrakten Wortbildungsparadigmen zusammenfassen zu können, müßten wir bereits über die Liste der Wortbildungskategorien des betreffenden Sprachsystems verfügen; und diese Kategorien sollen ja beim paradigmatischen Vorgehen erst durch funktionale Oppositionen innerhalb der Wortbildungsparadigmen ermittelt werden. 
Eine Parallele aus einer anderen Sprachebene mag diesen Gedanken verdeutlichen: im Bereich der Phonologie stellt die Annahme, daß dem An-, dem In- und dem Auslaut jeweils dasselbe Phonemsystem zugrundeliegt, eine je nach Sprache mehr oder weniger plausible, jedoch letzten Endes unbeweisbare Annahme dar, wie die Anhänger der sog. Prosodischen Schule betont haben: cf. etwa die Bemerkungen J.R. FIRTHS zu den Nasalen des Marathi (ein ähnliches Argument ließe sich unschwer für die Nasalvokale des Polnischen konstruieren) in FIRTH 1957: 51 (zur Prosodischen Schule allgemein cf. FISCHER-JøRGENSEN 1975: 59-63).

Vor uns liegt der typische Fall einer methodischen Antinomie. Sowohl die syntagmatische als auch die paradigmatische Methode der Wortbildung sind stark im Angriff, wenn sie auf die unbewiesenen Voraussetzungen der anderen Methode hinweisen; sie können sich jedoch selbst auf ihrem eigenen Terrain gegen den Vorwurf des Apriorismus nicht zur Wehr setzen. Ihr gegenseitiges Verhältnis sei durch folgendes Diagramm erläutert:

konkrete Wortbildungsparadigmen

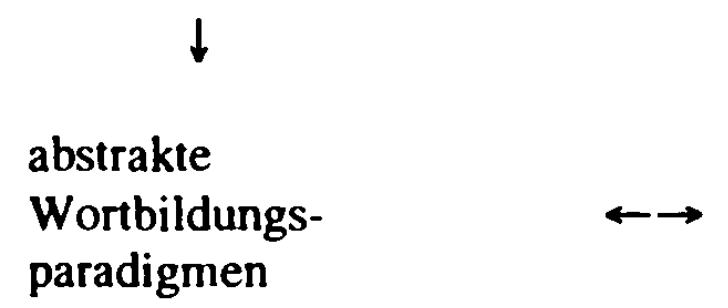

konkrete

Wortbildungspaare

Wortbildungskategorien ("abstrakte Wortbildungspaare")

Die Grundformel der syntagmatischen Methode lautet:

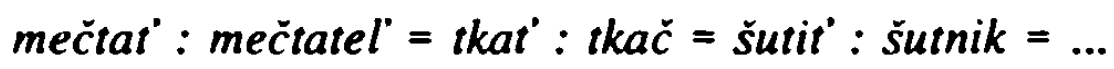

Die Grundformel der paradigmatischen Methode lautet:

$\frac{\text { plavil'ščik }}{\text { plavil'nik }}=\frac{\text { prjadil'ščik }}{\text { prjalka }}=\frac{\text { pajal'ščik }}{\text { pajal'nik }}=\ldots$

Die beiden senkrechten Pfeile zeigen, an welchen Punkten jede Methode ihre apriorischen Entscheidungen treffen muß: bei der syntagmatischen Methode ist dies der Übergang vom Wortbildungspaar zur Wortbildungskategorie (dem "abstrakten Wortbildungspaar"); bei der paradigmatischen Methode -- der Übergang vom konkreten zum abstrakten Wortbildungsparadigma. Die beiden waagrechten Pfeile stehen hingegen für Operationen, bei denen die beiden grundlegenden Einheiten des Wortbildungssystems -- das abstrakte Wortbildungsparadigma und die Wortbildungskategorie -- nach rein strukturimmanenten Kriterien abgeleitet werden: im Rahmen der 
paradigmatischen Methode können, wie bereits dargelegt, die Wortbildungskategorien mit Hilfe funktionaler Oppositionen nach dem Kriterium der Relevanz bestimmt werden; aber auch die syntagmatische Methode erlaubt eine entsprechende Operation: hat man erst einmal die Wortbildungskategorien festgelegt und ihnen alle Ableitungen eines bestimmten Wortschatzbereichs zugeordnet, so kann man dann in einem zweiten Schritt die konkreten Wortbildungsparadigmen auf Grund der Wortbildungskategorien, die in ihnen vorkommen, zu Einheiten höherer Abstraktionsstufen (abstrakten Wortbildungsparadigmen) zusammenfassen. Man erreicht auf diese Weise eine Einteilung der Wörter nach ihren Wortbildungseigenschaften, die nicht unmittelbar auf lexikalisch-semantische, syntaktische oder (flexions-)morphologische Kriterien zurückgreift.

Man sieht: jede der beiden Methoden liefert im Ergebnis jene Einheiten, die die Gegenmethode als ihren Ausgangspunkt voraussetzen muß. Es ist reizvoll, über ein rekursives und vielleicht auch selbstkorrigierendes Verfahren zu spekulieren, bei dem der Output der syntagmatischen Wortbildungslehre zum Input der paradigmatischen Wortbildungslehre gemacht wird und vice versa. Falls dieses Verfahren für einen bestimmten Wortschatzausschnitt unabhängig von den gewählten Ausgangsgrößen stets gegen dasselbe Inventar von Wortbildungskategorien bzw. abstrakten Wortbildungsparadigmen konvergiert, so wäre der höchste Grad an Objektivität erreicht, der von einer Wortbildungsanalyse zu erwarten ist. Bevor man freilich an diese Aufgabe herangehen kann, müßte das Instrumentarium der bislang vernachlässigten paradigmatischen Methode in mehreren Einzeluntersuchungen verfeinert werden. Jedenfalls darf man getrost die syntagmatische und die paradigmatische Methode einander gegenüberstellen, ohne der einen oder der anderen den Vorzug zu geben: dem Vorwurf des Eklektizismus setzt man sich dadurch keineswegs aus.

Wir schließen unsere Methodendiskussion mit einer kurzen Bemerkung, die über den Rahmen der Wortbildungslehre hinausgeht. Wie man unschwer einsieht, setzt sich der Gegensatz von syntagmatischer und paradigmatischer Methode auch in der Syntax fort: dem syntagmatischen Verfahren der Kasusgrammatik, die von einem a priori vorgegebenen System von Kasusrollen ausgeht, kann man hier das paradigmatische Verfahren der Valenztheorie gegenüberstellen, bei dem das Rektionsmodell (das "synraktische Paradigma") des Einzelverbs (oder einer Gruppe von Verben) am Anfang der Betrachtungen steht.

Die Gegenüberstellung der syntagmatischen und der paradigmatischen Methode der Wortbildungslehre erlaubt uns nun, die Fragestellung der vorliegenden Untersuchung zu formulieren. Wir wollen die paradigmatische Methode auf die Beschreibung eines Ausschnittes der desubstantivischen Wortbildung des Russischen anwenden -- auf Ableitungen russischer Substantive, deren Denotate konkrete Gegenstände wie Pfanne, Kanarienvogel oder Tomate sind. Wir haben für unsere Untersuchung Substantive gewählt, deren Denotate in eine der folgenden fünf Kategorien fallen: 
frukty (Obst), ovošči (Gemüse), pticy (Vögel), posuda (Geschirr und Besteck) sowie sredstva peredviženija (Fortbewegungsmittel). (Die Substantive, die solche Gegenstände bezeichnen, werden in der Sekundärliteratur meist mit dem leicht mißverständlichen Terminus konkrete Substantive bezeichnet; man kann dann die Substantive, die Gegenstände einer bestimmten Kategorie denotieren, zu einer Kategorie konkreter Substantive zusammenfassen; mehr dazu im nächsten Kapitel). Die Auswahl dieses Gegenstandsbereiches erlaubt es, auf die bislang in Zusammenhang mit der Wortbildung wenig behandelten Probleme der Kategorisierung einzugehen; er ist (und das ist vielleicht ganz gut so) für die Anwendung der paradigmatischen Methode keineswegs in höherem Maße geeignet als andere.

Unsere Arbeit geht demnach von der Annahme aus, daß die Substantive jeder einzelnen dieser fünf Kategorien in ihren Ableitungen dasselbe Inventar von Wortbildungskategorien realisieren, d.h. daß im Bereich der desubstantivischen Wortbildung des Russischen zwischen abstrakten Wortbildungsparadigmen und natürlichen Kategorien eine Entsprechung besteht. Dieser Grundannahme kommt dabei eine doppelte Funktion zu: sie ist einerseits das Postulat, das die paradigmatische Wortbildungsanalyse überhaupt erst in Bewegung setzt (Kapitel 1.3); andererseits eine Hypothese, die mit Hilfe der Ergebnisse gerade dieser Wortbildungsanalyse auf ihre Stichhaltigkeit hin zu überprüfen ist (Kapitel 4.6).

Schließlich sei noch kurz begründet, warum wir den Geltungsbereich unserer abstrakten Wortbildungsparadigmen nach den Prinzipien der natürlichen Kategorisierung und damit nach einem lexikalischen Kriterium zusammenstellen. Daß im Bereich der konkreten Substantive, die allenfalls eine rudimentäre Aktantenstruktur besitzen, eine syntaktische Einteilung wenig Sinn macht, liegt auf der Hand. Aber auch die Annahme, die gesamte Wortart Substantiv sei im Russischen (oder in irgendeiner anderen Sprache) am elegantesten durch ein einziges abstraktes Wortbildungsparadigma zu erfassen, scheint nicht besonders plausibel. Vergessen wir nicht, daß das Substantiv semantisch die heterogenste Wortart darstellt, da es Wörter aller übrigen Wortarten in sich aufnehmen kann (s. den anregenden Aufsatz von LÖNNGREN 1981a). Cf. dazu die Bemerkungen von ZEMSKAJA 1978: 72:

Naibol'šee različie nabljudaetsja meždu paradigmami slov raznyx častej reči. Odnako i v predelax odnoj časti reči imejutsja suščestvennye rasxoždenija meżdu paradigmami slov raznyx leksiko-semantičeskix grupp. Tak, naprimer, esli my vożmem $v$ kačestve proizvodjaščix imena suščestvitelnye so značeniem lica, konkretnogo predmeta $i$ otvlečennogo ponjatija, to uvidim, čto nabor proizvodnyx u nix budet različat'sja ves'ma sil'no.

$\mathrm{Zu}$ einem ähnlich negativen Ergebnis hinsichtlich der Homogenität der Kategorie der Substantive ist SCHUPBACH 1975: 186sqq. gelangt. Dies ist umso interessanter, als sein Ausgangspunkt in der Annahme der Einheitlichkeit der desubstantivischen 
Wortbildung bestand: nur so ist seine Entscheidung erklärbar, eine Zufallsauswahl von ca. 200 substantivischen Wortfamilien zu untersuchen. ${ }^{11}$ Mit der Bearbeitung von Substantiven bestimmter lexikalisch-semantischer Kategorien ordnen wir uns in eine Forschungstradition ein, die vor allem durch die Dissertationen der Schüler von E.A. ZEMSKAJA vertreten wird (cf. die Aufzählung bei ZEMSKAJA 1992: 18, Anm. 2).

Die Beschreibung des gewählten Gegenstandsbereiches soll in dieser Arbeit in folgenden Schritten vorgenommen werden:

Es wurde oben bereits ausgeführt, daß die paradigmatische Methode bei der Bestimmung ihrer Ausgangseinheit, des abstrakten Wortbildungsparadigmas, auf andere Sprachebenen zurückgreifen muß, und daß wir uns entschlossen haben, hierbei ein lexikalisch-semantisches Kriterium anzuwenden: wir gehen davon aus, daß die konkreten Substantive jeder einzelnen der aufgezählten Kategorien in ihren Ableitungen jeweils dasselbe abstrakte Wortbildungsparadigma realisieren. Kapitel 1 ist der Frage nach dem Bestand und dem inneren Aufbau solcher Kategorien wie Fortbewegungsmittel, Geschirr und Besteck oder Vögel gewidmet; es liegt auf der Hand, daß wir uns hier, wo es lediglich darum geht, den Geltungsbereich unserer abstrakten Wortbildungsparadigmen zu bestimmen, noch außerhalb des Bereiches der eigentlichen Wortbildungslehre befinden. Da sich jedoch die kategoriale Struktur der Substantive des Russischen auch in deren Ableitungen manifestiert, werden viele Begriffe, die im ersten Kapitel eingeführt werden, auch im weiteren Fortgang der Untersuchung benötigt.

Im Mittelpunkt von Kapitel 2 steht der Begriff des Wortbildungsparadigmas. Es wird ein Algorithmus zur Bestimmung des Wortbildungsparadigmas konkreter Substantive vorgeschlagen. Der zentrale Gedanke besteht darin, das Wortbildungsparadigma nicht positiv, sondern negativ abzugrenzen: den A usgangspunkt bilden nicht die Wörter, die von dem betreffenden Substantiv direkt, sondern diejenigen, die von ihm indirekt abgeleitet sind; die restlichen Wörter der Wortfamilie werden dann eo ipso zum Wortbildungsparadigma des Ausgangssubstantivs gerechnet. Dadurch werden die Schwierigkeiten, die die Aufstellung von Ableitungsbeziehungen besonders auf der ersten Ableitungsstufe bereitet, auf elegante Weise umgangen.

"Ob eine Auswahl, die sich auf einen bestimmten Ausschnitt des Alphabets ("from vremja through $2 n o j$ ") beschränkt und einige Substantive auf Grund der semantischen Komplexität ihrer Wortfamilien einfach aus dem Untersuchungskorpus ausschließt, noch einen Anspruch auf Zufälligkeit erheben kann, darf allerdings bezweifelt werden. 
In Kapitel 3 werden die allgemeinen Prinzipien der paradigmatischen Wortbildungsanalyse: das Vollständigkeits-, das Ökonomie- und das Natürlichkeitsprinzip vorgestellt und einige allgemeine Probleme der desubstantivischen Wortbildung behandelt. Die dialektische Theorie der Metapher, die in diesem Kapitel vorgestellt wird, erlaubt uns, in der herkömmlichen Gegenüberstellung von Modifikation und Mutation einen Gegensatz zwischen metaphorischen und metonymischen Ableitungen zu erkennen.

- Kapitel 4 ist schließlich der Analyse der konkreten Wortbildungsparadigmen der Substantive der Kategorien frukty, ovošči, pticy, posuda und sredstva peredviženija gewidmet. Jeder der vier ersten Kategorien wird ihr eigenes abstraktes Wortbildungsparadigma zugeordnet; im Fall der fünften Kategorie wird begründet, warum auf die (von theoretischem Standpunkt aus zulässige) Aufstellung eines eigenen abstrakten Wortbildungsparadigmas verzichtet wird. 


\section{WÖRTER FÜR SACHEN}

Im Zentrum dieses Kapitels stehen die Begriffe des konkreten Substantivs und der (natürlichen) Kategorie. Abschnitt 1.1 ist dem ersten dieser Begriffe, Abschnitt 1.2 dem zweiten gewidmet; in Abschnitt 1.3 wird dargelegt, mit welcher Methode die Substantive gewonnen wurden, die in dieser Arbeit auf ihre Wortbildungseigenschaften hin untersucht werden sollen. Wie bereits in der Einleitung erwähnt, handelt es sich dabei um Substantive, deren Denotate einer der folgenden fünf Kategorien angehören:

- frukty (Obst)

- ovošči (Gemüse)

- posuda (Küchengeschirr und Besteck)

- pticy (Vögel) und

- sredstva peredviženija (Fortbewegungsmittel).

\subsection{Konkrete Substantive: Wörter oder Sachen?}

Die aufgezählten Kategorien vermitteln eine erste Vorstellung davon, was hier mit dem Terminus konkretes Substantiv (Konkretum) gemeint ist: Substantive, mit denen man sich auf die materiellen (nicht unbedingt leblosen) Objekte beziehen kann, die uns im Alltag umgeben. Personenbezeichnungen, die meist ebenfalls zu den Konkreta gezählt werden, lassen wir beiseite, da sie sich in erster Linie auf bestimmte soziale Rollen und nur indirekt auf deren physische Träger beziehen. Damit umfassen die Konkreta sowohl agentivische (Tiere!) als auch inagentivische Substantive (nach der Einteilung bei TROST 1992).

Neben dem Terminus konkretes Substantiv (konkretnoe suščestvitel'noe) ist der Terminus gegenständliches Substantiv (predmetnoe suščestvitel'noe) in Gebrauch. Wenn hier dem ersteren der Vorzug gegeben wird, so allein deshalb, weil er das bequeme Univerbat Konkretum neben sich hat und sich mit der Einbeziehung von Tierbezeichnungen besser verträgt als der zweite. Beide Termini sind insoferm mißverständlich, als ja die Eigenschaften "konkret" bzw. "gegenständlich" nicht den Substantiven selbst, sondern ihren Denotaten zukommen. Die Gefahr einer Verwechslung von Wort und Sache ist, wie noch zu erläutern sein wird, bei der Untersuchung der konkreten Substantive besonders groß; wir haben uns trotzdem nicht entschließen können, an diesem Punkt vom überkommenen terminologischen Sprachgebrauch abzuweichen -- umso mehr, als dieser bereits in die allgemeinsprachigen Wörterbücher Eingang gefunden hat: die neueste Auflage des beliebten Wörterbuches von S.I.OżEGOV und N.JU.ŚVEDova (ŚVE) enthält unter dem Stichwort konkretnyj folgenden Hinweis: 
konkretnye imena suščestvitel'nye -- $v$ grammatike: suščest vitel'nye, nazyvajuščie ediničnye material'nye, veščestvennye realii, otdel'nye predmety, lica (napr., karandaš, dom, jabloko, inžener, čudak).

Als charakteristische sprachliche Eigenschaften konkreter Substantive gelten die Abwesenheit einer Aktantenstruktur sowie die Möglichkeit, durch Ostension definiert zu werden (FRUMKINA et al. 1991: 7 oder MostovaJA 1990: 143). Beides gilt nur bedingt: Artefakte z.B. vermitteln sehr wohl die Vorstellung einer bestimmten Situation mit den darin vorkommenden Teilnehmerrollen (man denke an tramvaj oder noz); und unzweideutige ostensive Definitionen sind allein unter der Voraussetzung möglich, daß sich die beiden Gesprächsteilnehmer über das gemeinte Abstraktionsniveau im voraus einig sind: dem Verweis auf einen Hund ist ja als solchem nicht zu entnehmen, ob er der Erklärung von taksa, sobaka oder životnoe dienen soll.

Im folgenden werden nicht alle konkreten Substantive des Russischen behandelt, sondern lediglich eine sehr begrenzte Auswahl von etwas mehr als 200 Wörtern, an deren Zugehörigkeit zu den Konkreta kein Zweifel besteht. Wir begnügen uns deshalb mit diesen kurzen Hinweisen, ohne die mühevolle, wenn nicht sogar unlösbare Aufgabe einer vollständigen Klassifizierung der russischen Substantive nach semantischen Gesichtspunkten in Angriff zu nehmen (cf. FrUMKINA et al. 1991: 12, Anm. 1).

Zwei Dimensionen sind für die Analyse der Inhaltsseite konkreter Substantive besonders wichtig:

a) sprachlich (lexikalisch) vs. enzyklopädisch: das sprachliche (lexikalische) Wissen umfaßt die Merkmale, die in die Definition eines Wortes eingehen; das enzyklopädische Wissen umfaßt die Eigenschăften der Gegenstände, auf die man sich mit dem betreffenden Wort beziehen kann. (Cf. VIEHWEGER 1977: 284-287, wo sprachliches Wissen als "systemhaft relevant gewordenes Sachwissen" [286] definiert wird.) Diese Unterscheidung führt die traditionelle Unterscheidung von (sprach)analytischen und (sprach)synthetischen Urteilen fort: wenn man sich z.B. dafür entschieden hat, russ. kaktus als 'tropičeskoe rastenie s mjasistymi stebljami, v koljučkax i bez list'ev' zu definieren (UŠA), so gehört das Merkmal 'bez list'ev' in die sprachliche Zone der Inhaltsseite von kaktus, und der Satz "U kaktusov koljučki vmesto list'ev" ist bereits auf Grund der Definition von kaktus wahr: ein Kaktus mit Blättern wäre in diesem Fall keine botanische Abnormität, sondern eine sprachliche Unmöglichkeit. Die Information, daß Kakteen nur in den Wüsten der Neuen Welt vorkommen, ist dagegen enzyklopädisch: über den Wahrheitsgehalt des Satzes "Kaktusy rastut tolko v Amerike" entscheidet nicht die russische Sprache, sondern der Augenschein. 
Die Unterscheidung zwischen sprachlichem (lexikalischem) und enzyklopädischem Wissen ist in den letzten Jahrzehnten in zunehmendem Maße unter Beschuß geraten; daß die Grenze zwischen den beiden Bereichen nur willkürlich gezogen werden kann, gehört zu den zentralen und inzwischen bis zum Überdruß wiederholten Glaubenssätzen der sog. kognitiven Sprachwissenschaft: charakteristische Äußerungen finden sich etwa bei HUDSON 1990: 274 oder auch GEERAERTS 1989: 588sq., eine eingehende Diskussion der Problematik bei HaIman 1980 und GeERAERTS 1985. Wir werden sehen, daß gerade konkrete Substantive gewisse Argumente dafür liefern, die Gültigkeit der Unterscheidung einer sprachlichen und einer enzyklopädischen Wissenskomponente in Frage zu stellen.

b) naiv vs. wissenschaftlich: naives Wissen ist das Wissen, mit dem wir im Alltag operieren; wissenschaftlich ist das Wissen, das den Anforderungen der neuzeitlichen Erkenntninistheorie in der Nachfolge von BACON und DESCARTES genügt und im modernen Wissenschaftsbetrieb institutionell verankert ist. Naives Wissen findet sich in allen Kulturen; das wissenschaftliche Wissen ist für die Kultur der Neuzeit charakteristisch, die von Westeuropa ihren Ausgang nahm und sich inzwischen über den ganzen Erdball verbreitet hat.

Diese beiden Begriffspaare werden sehr oft in einem Atemzug genannt (USA p. XXIV, § 7) oder sogar miteinander verschmolzen, indem man das sprachlich-naive Wissen dem enzyklopädisch-wissenschaftlichen gegenüberstellt (APRESJAN 1995, Index, s.v. énciklopedičeskij, naivnyj). Der Unterschied zwischen der sprachlichen und der enzyklopädischen Dimension unseres Wissens ist jedoch mit dem Gegensatz zwischen dem naiven und dem wissenschaftlichen Weltbild keineswegs deckungsgleich (cf. HARTENSTEIN 1981: 94-97 und 110-113): einerseits umfaßt ja das enzyklopädische Wissen eines durchschnittlichen Sprachbenutzers nicht nur wissenschaftlich überprüfte Tatsachen; andererseits muß jede Wissenschaft ihre Termini definieren, bevor sie an die Untersuchung der Gegenstände herangehen kann, auf die man sich mit ihnen beziehen kann, und besitzt somit auch eine (fach)sprachliche Dimension. Die Identifizierung von "enzyklopädisch" und "wissenschaftlich", die die Identifizierung von "naiv" und "sprachlich" automatisch nach sich zieht, wird durch die Tatsache begünstigt, daß enzyklopädische Nachschlagewerke in der westlichen Kultur nur die wissenschaftliche Beschreibung der Gegenstände enthalten und nicht die "current platitudes" (MAX BLACK 1962: 40), die man mit ihnen assoziiert; man darf sich jedoch von dieser durchaus kulturspezifischen Tatsache nicht irreleiten lassen. (Wir brauchen hier nicht auf die Frage einzugehen, auf welche Weise die Unterscheidung zwischen naivem und wissenschaftlichem Wissen auf traditionelle Kulturen ausgedehnt werden könnte; man müßte in diesem Fall wahrscheinlich von Fach- und Laienwissen sprechen.)

Bei der Behandlung der Semantik konkreter Substantive zeigt sich allerdings sehr bald, daß sprachliche (lexikalische) und enzyklopädische Wissenskomponenten in 
vielen Fällen nur auf künstliche Weise voneinander getrennt werden können; in einer modernen, technisierten Gesellschaft mit allgemeiner Schulpflicht dürfte auch der Gegensatz zwischen dem naiven und dem wissenschaftlichem Weltbild allmählich seine Schärfe verlieren. Wir wollen hier nur auf den ersten dieser beiden Aspekte eingehen.

Daß in der Inhaltsseite vieler konkreter Substantive sprachliches und enzyklopädisches Wissen auf unentwirrbare Weise miteinander verflochten sind, zeigt sich daran, daß man mit diesen Substantiven kaum echte (sprach)analytische Urteile bilden kann: der Unterschied zwischen den Aussagen, daß Kakteen keine Blätter aufweisen und daß sie ausschließlich in Amerika verbreitet sind, ist in dieser Hinsicht weitaus weniger deutlich, als oben zu Illustrationszwecken angenommen wurde. Angesichts dieser Schwierigkeit war man versucht, konkrete Substantive wie Eigennamen zu behandeln, d.h. ihnen eine rein sprachliche Inhaltsseite abzustreiten, um sie aus dem Gegenstandsbereich der lexikalischen Semantik ganz zu verbannen.'

Wir wollen hier einen weniger radikalen Weg einschlagen und an der Grenze zwischen lexikalisch relevanten Merkmalen und enzyklopädischen Tatsachen festhalten. Im Bereich der konkreten Substantive ist jedoch diese Grenze extrem beweglich: ein und dieselbe Wissenskomponente kann bald als definitorisches Merkmal, bald als Eigenschaft des Gegenstandes selbst aufgefaßt werden. ${ }^{2}$ Gerade in der Wortbildung zeigt sich dies mit besonderer Deutlichkeit: so kommt etwa dem Merkmal 'želtyj' von limon in der Ableitung limonnica 'babočka limonno-želtogo ili zelenovato-belogo cveta' sprachimmanenter Status zu (cf. ISAĆENKO 1976: 423sqq.; APRESJAN 1992: 53); heißt das aber, daß sich die Fügung sinij limon auf dieselbe Weise wie z.B. die Fügung požiloj rebenok verhält -- daß sie die semantischen Kompatibilitätsregeln des Russischen verletzt und nur eine metaphorische Interpretation zuläßt?

Diese Plastizität der sprachlichen Inhaltsseite ist nicht für alle konkreten Substantive in demselben Maße charakteristisch. Am deutlichsten tritt sie bei Tier- und Pflanzen-

' WierzBicka 1972: 22. Die Autorin hat freilich an diesem Punkt ihre Meinung inzwischen geändert, s. WIERZBICKA 1985: 163. Auch im Modell 'Smysl $\leftrightarrow$ text' werden die konkreten Substantive von dem Kernbereich sprachwissenschaftlicher Untersuchungsgegenstände ausdrücklich ausgenommen; cf. HARTENSTEIN 1981: 108-110 und die Polemik bei Frumkina 1984: 6sqq.

${ }^{2}$ Ein einprägsames Bild dafür findet sich bei WITTGENSTEIN 1984: 284sq.; cf. WITTGENSTEIN 1992: 140, 151. IORDANSKAJA/MEL'ČUK 1980: 200-202 stellen zwei Tests auf, mit denen man konventionalisierte enzyklopädische Wissenskomponenten, die sie Konnotationen nennen, von sprachlich-lexikalischen Merkmalen unterscheiden kann; APRESJAN 1992: 49 fügt einen dritten Test hinzu, bemerkt aber selbst: "Ni odin iz ètix testov ne javljaetsja absoljutno nadežnym". 
bezeichnungen hervor -- und hier wiederum im mittleren, der Willkür sprachlicher Kategorisierung am wenigsten ausgesetzten Bereich der Taxinomie; bei jenen Konkreta also, deren Umfang uns gewissermaßen von der Natur aufgedrängt wird, deren Begriffsinhalt jedoch nur durch Beobachtung und wissenschaftliche Forschung aufgedeckt werden kann. Bei Artefakten bildet die Funktion den begrifflichen Kern, um den sich die enzyklopädische Information gruppiert; aber auch hier können einzelne vom funktionalen Standpunkt aus irrelevante Merkmale definitorischen Status erhalten und sich etwa in Ableitungsbeziehungen niederschlagen (paroxod, čugunok). Personenbezeichnungen (Eigennamen natürlich ausgenommen), die wir hier aus dem Kreis der konkreten Substantive ausgeschlossen haben, weisen schließlich in ihrer sprachlichen Inhaltsseite den höchsten Grad an Stabilität auf. Man hat diesen Unterschieden durch die Gegenüberstellung von identifizierenden und prädizierenden Wörtern (identificirujuščie und predikatnye slova) bzw. natural und nominal kinds Rechnung zu tragen versucht. ${ }^{3}$ Selbstverständlich handelt es sich hierbei nicht um eine starre Dichotomie, sondern um ein Kontinuum, in dem sehr viele Zwischenstufen möglich sind (cf. KEIL 1989: 56); um jedoch zu eindeutigen Entscheidungen zu gelangen, werden wir in dieser Arbeit lediglich den Tier- und Pflanzenbezeichnungen bei der Aufstellung von Ableitungsbeziehungen (Kapitel 2.2.3) bzw. bei der Analyse der Wortbildungsparadigmen (Kapitel 3.1) einen gewissen Sonderstatus einräumen.

\subsection{Grundfragen der natürlichen Kategorisierung}

Man hat die semantische Struktur der identifizierenden Wöner (und damit auch der konkreten Substantive) als diskret und autonom charakterisiert: der Referenzbereich (Umfang) des einen wird von Veränderungen im Referenzbereich des anderen kaum beeinflußt (ARUTJUNOVA 1980: 173sq.). Das bedeutet natürlich nicht, daß sie vollkommen beziehungslos nebeneinanderstehen und den semantischen Raum gleichsam als amorphe Masse ausfüllen; ihre Kristallisationsform scheint jedoch weniger das Trier'sche Wortfeld mit der strengen Wechselbestimmtheit seiner Glieder als das komplexe Stufengefüge naiver Taxinomien und Partinomien zu sein. Über deren Aufbau ist in den letzten Jahrzehnten eine Fülle von Publikationen erschienen, deren gemeinsamer Ausgangspunkt die Einsicht ist, daß die sogenannte aristotelische Logik mit ihren starren Regeln der Biegsamkeit der Kategorien des Alltagsdenkens nicht

${ }^{3}$ S. ARUTJUNOVA 1976: 326-356, ARUTJUNOVA 1980 (identificirujuščie vs. predikatnye slova) und SCHWARTZ 1978, SCHWARTZ 1980 (nominal vs. natural kinds). Cf. die Gegenüberstellung von constructive und substantive definitions bei BOLINGER 1965. Von kognitivem Standpunkt aus werden die Unterschiede zwischen natural kinds und artifacts bei ATRAN 1987 und KEIL 1989 analysiert. Die philosophische Literatur zu diesen Fragen ist unüberschaubar; einen guten Einstieg bietet der Sammelband SCHWARTZ (ed.) 1977. 
gerecht werden kann. ${ }^{4} \mathrm{Da}$ zu diesem Fragenkreis inzwischen in vielen Sprachen einführende Darstellungen vorliegen (KLEIBER 1993, DUBOIS (ed.) 1991, TAYLOR 1989, MILLER 1991: 171-189; cf. auch die einflußreiche Arbeit von LAKOFF 1987), werden wir hier nur eine sehr knappe Übersicht geben, wobei der Akzent auf weniger bekannten Beiträgen aus der russischen Forschungstradition liegen soll.

Wie so viele andere Grundbegriffe, ist auch der Begriff der Kategorie nur sehr schwer zu explizieren; bereits eine so vage Definition wie bei RosCH et al. 1976: 383 "a number of objects which are considered equivalent" erweist sich als zu eng, da, wie noch zu zeigen sein wird, manche Kategorien nicht nur durch Äquivalenzrelationen zusammengehalten werden: Messer und Gabel gehören zu derselben Kategorie, weil sie einander ergänzen und nicht, weil sie gegeneinander ausgetauscht werden können. Die Kategorien des Alltagsdenkens werden im Gegensatz zu den bewußt gebildeten Kategorien der Wissenschaft bzw. der psychologischen Forschung als natürliche Kategorien bezeichnet. Der Unterschied zwischen den Gegenständen der Außenwelt, die zu einer Kategorie zusammengefaßt werden, und den Unterkategorien, die in ihr enthalten sind, braucht in den folgenden Ausführungen, in denen es meist um das Verhältnis zwischen einer Kategorie und ihren Unterkategorien geht, nicht gekennzeichnet zu werden; es ist ohnehin fraglich, ob diesem Unterschied im Bereich natürlicher Sprachen dieselbe Bedeutung wie in der Mathematik (Elementvs. Inklusionsbeziehungen) oder der traditionellen Logik (Spezies vs. Individuum) zukommt (cf. JACKENDOFF 1983: 88-91, 95-100; CulıLı 1990: 47). Für den Ausdruck Kategorie(n) konkreter Substantive gilt dasselbe, was oben über den Terminus konkretes Substantiv gesagt worden ist: strenggenommen sind es nicht die Substantive, sondern deren Denotate, die kategorisiert werden; die Kategorisierung der Denotate schlägt sich dann indirekt in der Kategorisierung der Substantive selbst nieder. Statt von Kategorien werden wir in den folgenden Ausführungen gelegentlich auch von Begriffen sprechen; der erste Terminus unterstreicht den extensionalen, der zweite den intensionalen Aspekt des Kategorisierungsprozesses.

\subsubsection{Horizontale und vertikale Profilierung natürlicher Kategorien}

Die Mitglieder der Kategorien unseres Alltagsdenkens haben nicht alle das gleiche Gewicht. Diese Ungleichmäßigkeit kann im psycholinguistischen Experiment mit zweierlei Methoden nachgewiesen werden (cf. ECKES 1991: 102-106):

a) direkte Urteilsmethoden: die Versuchspersonen geben dabei auf einer (meist fünf- oder siebenstufigen) Skala an, wie typisch einzelne Mitglieder einer Kategorie

"Die Bezeichnung "aristotelisch" wird freilich Aristoteles, in dessen Denken der Begriff der Kontinuität eine große Rolle spielt (LOVEJOY 1960: 55-58), nicht gerecht; zu deren Ursprüngen cf. KAKRIDIS 1993: 115, Anm. 5. 
für diese Kategorie als Ganzes sind. Die beurteilte Größe wird als Typikalität bezeichnet. Zur Illustration führen wir die Instruktion eines Experimentes an, das im deutschsprachigen Raum durchgeführt wurde:

In dieser Untersuchung geht es um die Frage, wie die Beziehung verschiedener Objekte zu der Kategorie gesehen wird, der sie angehören. Nehmen wir z.B. die Kategorie "HUND". Ein Deutscher Schäferhund wird den meisten von uns als ein typischeres Mitglied der Kategorie der Hunde erscheinen als etwa ein Pekinese oder ein Mops. Oder nehmen wir die Kategorie "BLUME". Die meisten von uns werden darin übereinstimmen, daß eine Rose ein sehr typisches Exemplar dieser Kategorie ist, während ein Kaktus eher als eine untypische Blume bezeichnet werden kann.

Auf den folgenden Seiten werden Sie gebeten zu beurteilen, wie typisch verschiedene Objekte für ihre jeweilige Kategorie sind. [...] Lassen Sie sich im folgenden nicht davon leiten, wie gut oder schlecht Ihnen das betreffende Objekt gefällt. Es geht ganz allein um Ihr persönliches Urteil, wie typisch die Objekte sind. (ECKES 1985: 194sq.)

Diese experimentelle Methode geht auf die amerikanische Psychologin E. RosCH zurück. Es zeigt sich, daß die Versuchspersonen diese Art der Aufgabenstellung verständlich finden und bei ihren Beurteilungen (zumindest, was die beiden Extrempunkte der Skala betrifft) zu recht einheitlichen Ergebnissen gelangen (RoSCH 1977: 22sq., zu der Frage der Stabilität von Typikalitätsurteilen aber auch ECKES 1991: 30 und die dort zitierte Literatur).

b) Produktionsmethoden: mit ihnen kann die Exemplar-Dominanz (MitgliederDominanz) oder die Kategorien-Dominanz eines Begriffes gemessen werden, d.h. die Stärke, mit der verschiedene untergeordnete bzw. übergeordnete Begriffe mit ihm assoziiert werden. Für die Ermittlung der Exemplar-Dominanz wird der Name einer übergeordneten Kategorie vorgegeben; die Versuchspersonen werden gebeten, in einem bestimmten Zeitraum (z.B. 30 Sekunden) möglichst viele Mitglieder der betreffenden Kategorie zu notieren. Sehr bekannt sind die Ergebnisse eines großangelegten Produktionsexperimentes, das BATTIG und MONTAGUE zur ExemplarDominanz in 56 verbalen Kategorien des Englischen durchgeführt haben (BATTIG/MONTAGUe 1969); ein deutsches Beispiel ist die Studie von MANNHAUPT 1983. Für die Ermittlung der Kategorien-Dominanz scheint es keine allgemein anerkannte experimentelle Methode zu geben; cf. die Zusammenfassung bei ECKES 1991: 105sq.

Zwischen der Typikalität und der Exemplar-Dominanz besteht ein statistisch nachweisbarer Zusammenhang -- weniger stark, als man anfänglich angenommen hatte, aber doch stark genug, um an der grundsätzlichen Dualität von Zentrum und Peripherie in der Struktur natürlicher Kategorien keinerlei Zweifel aufkommen zu lassen (ROSCH 1973: 132sq., ECKES 1991: 105). Diese Dualität wird man wahrscheinlich als 
eine kulturelle Universalie betrachten dürfen. ${ }^{5}$ Für die zentralen Mitglieder einer Kategorie hat sich der Terminus Prototyp eingebürgert, dessen Bedeutung freilich in dem nicht anders als inflationär zu bezeichnenden Sprachgebrauch der letzten Jahre immer mehr verschwimmt (diese Entwicklung wird bereits von MEDIN/SMITH 1981: 169 beklagt).

Die Herausbildung von Prototypen haben RosCH und ihre Mitarbeiter im Anschluß an WITTGENSTEINS Metapher von den Familienähnlichkeiten folgendermaßen erklärt: als besonders typisch werden jene Mitglieder einer Kategorie eingestuft, die die meisten gemeinsamen Merkmale mit den Mitgliedern ihrer eigenen Kategorie und die wenigsten gemeinsamen Merkmale mit Mitgliedern fremder Kategorien aufweisen (ROSCH/MERviS 1975; ROSCH 1977: 35-37). Gegen diese Erklärung wurde von GEERAERTS 1988 das Vorhandensein koextensionaler Kategorien mit unterschiedlicher Typikalitätsstruktur ins Feld geführt. GEERAERTS sieht in der prototypischen Struktur natürlicher Kategorien die Wirkung eines allgemeineren Prinzips, das zur Verbindung von structural stability mit flexible adaptability führt: die Unterscheidung zwischen (homogenem und daher festem) Kem und (heterogener und daher variabler) Peripherie gewährleistet, daß unsere Kategorien ohne großen kognitiven Aufwand den Erfordernissen einer sich verändernden Umwelt angepaßt werden können. Dieses Prinzip kann freilich nicht voraussagen, welche Mitglieder der Kategorie zu ihren Prototypen avancieren werden.

Auch auf der vertikalen Achse sind natürliche Kategorien einander nicht ebenbürtig: in einer hierarchischen Stufenfolge von Begriffen gibt es in der Regel einen, der größeres Gewicht als die übrigen besitzt und im spontanen Akt der Nomination (erste Nennung im Text, Antwort auf die Was-Frage) bevorzugt wird. Es handelt sich dabei meistens um Begriffe, die auf einer mittleren Abstraktionsebene, der sogenannten Basisebene (basic level), angesiedelt sind; bei biologischen Kategorien sind dies Bezeichnungen für einzelne Arten wie čerepaxa, koška, lev, lisica etc. RosCH et al. 1976 sind der Frage nachgegangen, wodurch sich die Begriffe der Basisebene von den Begriffen über- und untergeordneter Ebenen unterscheiden; sie gelangten dabei zu dem Ergebnis, daß die Kategorien der Basisebene die umfassendsten Kategorien sind, deren Mitglieder eine große Zahl gemeinsamer Eigenschaften aufweisen, zu ihrer Handhabung einheitliche motorische Programme erfordern und weitgehend deckungsgleiche Umrisse besitzen. Anders ausgedrückt: die Basisebene ist jene Kategorisierungsebene, auf der die Mitglieder einer Kategorie die meisten gemeinsamen Merkmale untereinander und die wenigsten gemeinsamen Merkmale mit Mitgliedern benachbarter Kategorien aufweisen, wodurch die totale Merkmals-Validität der Kategorien ihr Maximum erreicht (zur Berechnung der Merkmals-Validität s. Rosch et al. 1976: 384sq. und die kritische Diskussion bei ECKES 1991: 126-128).

${ }^{5} \mathrm{Cf}$. den Begriff der semiotischen Ungleichmäßigkeit (semiotičeskaja neravnomernost') bei LOTMAN 1992: 16sq. sowie DANES 1982 und GEERAERTS 1985. 
Bei übergeordneten Kategorien wird die Verallgemeinerung durch den entstehenden Informationsverlust wettgemacht; bei untergeordneten Kategorien bringt die zusätzliche Spezifikation keinen nennenswerten Informationsgewinn.

Sowohl die horizontale als auch die vertikale Profilierung natürlicher Kategorien haben eine Vielzahl weiterer Erklärungsversuche hervorgerufen, über die in den Überblicksdarstellungen von MEDIN/SMITH 1981, ECKES 1991, HOFFMANN 1986 und LAKOFF 1987 ausführlich referiert wird. Für die Fremdsprachenphilologien dürfte vor allem die Frage interessant sein, in welchem Maße Auswahl der Prototypen einer Kategorie und die Bestimmung der Basisebene von Kräften gesteuert werden, auf die wir als menschliche Wesen keinen Einfluß mehr haben (eine in sich bereits strukturierte Wirklichkeit; unbewußte kognitive Mechanismen), und in welchem Maße in sie auch subjektive, insbesondere kulturspezifische Faktoren eingehen. Je nachdem, wie die Akzente gesetzt werden, kann man unter diesem Gesichtspunkt zwischen objektivistischen und relativistischen Erklärungsmodellen unterscheiden. E. RoSCH schien in der Anfangsphase ihrer Arbeit eher der objektivistischen Interpretation der Typikalitäts- und Basisebeneneffekte zuzuneigen. ${ }^{6}$ Ihr Erklärungsmodell für die Herausbildung der Basisebene erinnert sehr stark an die Charakterisierung der Species durch den Klassifikationstheoretiker G.G. SIMPSON:

A group is nonarbitrary as to inclusion if all its members are continuous by an appropriate criterion, and nonarbitrary as to exclusion if it is discontinuous from any other group by the same criterion. It is arbitrary as to inclusion if it has internal discontinuities and as to exclusion if it has an external continuity. $|\ldots|$ Thus the point made by those who consider the species as objective and other taxa as subjective can be more clearly stated thus: the species (genetically defined) is the one taxon that is usually nonarbitrary both as to inclusion and as to exclusion (SIMPSON 1961: 115; das Kontinuitätskriterium, das der Autor an dieser Stelle aufstellt, ist allerdings nicht die Gemeinsamkeit von begrifflichen Merkmalen, sondern die Fähigkeit, miteinander Nachkommen zu zeugen).

Objektivistisch ist auch die Erklärung, die HofFMANN 1986: 72 für die Auswahl der Begriffe der Basisebene aufstellt, die von ihm Primärbegriffe genannt werden: es sind dies "die relativ allgemeinsten noch sensorisch repräsentierten Begriffe". Al-

'Der objektivistische Charakter von RosCHs Modellen wird etwa an Formulierungen wie RoSCH et al. 1976: 428 oder ROSCH 1977: 28sq. deutlich; cf. aber die differenzierte Darstellung in RosCH et al. 1976: 428-432. Zur Entwicklung von ROSCHs Auffassungen LAKOFF 1987: 42sq., zur Kritik FrUMKINA et al. 1991: 47sq., FRUMKINA/MIXEEV 1996: 55sq. Cf. die Gegenüberstellung von materialistic [physiological, referential, statistical] und functional /psychological] bei GEERAERTS 1988. 
lerdings nimmt derselbe Autor an einer späteren Stelle seines Buches eine verbesserte Begriffsbestimmung vor, die auch auf die funktionalen Eigenschaften der Gegenstände Rücksicht nimmt: "Die Existenz von Primärbegriffen ist das Resultat des glücklichen Zusammentreffens von einander begünstigenden perzeptiven und funktionalen Eigenschaften von Objektmengen. [...] Nur in der Tendenz sind die Primärbegriffe zugleich auch die relativ allgemeinsten noch sensorisch repräsentierten $\mathrm{Be}$ griffe. Funktionell notwendige Differenzierungen können durchaus dazu führen, daß auch sensorische Subbegriffe lernabhängig die Qualität von Primärbegriffen erwerben" (HOFFMANN 1986: 107sq.). Offensichtlich haben wir damit den Boden der rein objektivistischen Erklärungsversuche verlassen, da die funktionale Ausrichtung eines Gegenstandes in hohem Maße subjekt- und interessenbezogen ist: wo für den Bewohner einer russischen Großstadt die Charakterisierung eines bestimmten Lebewesens als ptica ausreicht, wird es ein erfahrener Jäger sofort als drozd, zjablik, ivolga etc. einordnen.?

Unsere Untersuchung, die sich ja im Rahmen einer einzigen Kultur- bzw. Sprachgemeinschaft bewegt, kann weder für die relativistische noch für die objektivistische Position Argumente liefern. Wir wollen deshalb hier die Frage, ob unsere Beobachtungen zur Struktur der Kategorien frukty, ovošči, pticy, posuda und sredstva peredviženija auch über die Grenzen des Russischen hinaus Gültigkeit beanspruchen dürfen, bewußt offenlassen und auf diese Kategorien weiterhin ausschließlich mit ihren objektsprachlichen Bezeichnungen referieren.

\subsubsection{Qualitative Aspekte der Struktur natürlicher Kategorien}

Im Forschungsansatz von E. RosCH, der bislang im Mittelpunkt unserer Ausführungen stand, wird die Struktur natürliçher Kategorien auf eine einzige, experimentell meßBbare Dimension reduziert. Außerdem behandelt er, wie WIERZBICKA 1985: 263sq. und FRUMKINA et al. 1991: 53sqq. zu recht bemängeln, perzeptuelle, biologische und funktionale Kategorien, ja sogar Konkreta und Abstrakta auf einheitliche Weise -obwohl es doch von vornherein wenig plausibel scheint, daß der innere Aufbau so unterschiedlicher Kategorien wie bird, sport und vehicle identisch ist, so daß etwa zwischen bird und robin dieselbe Beziehung wie zwischen sport und football, vehicle und car bestünde. $\mathrm{Zu}$ einer differenzierteren Sicht gelangt man, wenn man die qualitativen Aspekte der Kategorisierung heranzieht.

${ }^{7} \mathrm{Cf}$. den Bericht über die Basisbegriffe eines Flugzeugmechanikers in RosCH et al. 1976: 430sq. und den frühen Aufsatz von BROWN 1958, in dem der Autor das Prinzip der funktionalen Äquivalenz für die Wahl der Benennungsebene gegenüber Kleinkindern verantwortlich macht; zur Problematik allgemein auch DOUGHERTY 1981: 175-178, CRUSE 1977 und TVERSKY 1986. 
Ein fundamentales Einteilungskriterium liefert hier der Gegensatz zwischen metaphorischen und metonymischen Assoziationen, d.h. Ähnlichkeits- und Kontiguitätsassoziationen, wobei unter Kontiguität dreierlei zu verstehen ist: zeitliche Abfolge (Wochentage, Jahreszeiten), räumliche Nachbarschaft (Körperteile) und kausale Beziehungen der verschiedensten Art, die in der Regel durch einen Handlungsbegriff vermittelt werden (z.B. Teilnehmer, Ort und Requisiten eines Spiels; Computer, Bildschirm, Tastatur, Drucker; etc.). (Eine vertiefte Diskussion des Gegensatzes von Metonymie und Metapher wird in Kapitel 3.3 gegeben). Das Standardbeispiel für ein metaphorisch strukturiertes Kategoriensystem ist die Taxinomie (cf. den grundlegenden Aufsatz von KAY 1971); das Standardbeispiel für ein metonymisch strukturiertes Kategoriensystem ist die Partinomie (gelegentlich auch als meronomy bezeichnet, etwa bei CRUSE 1986; cf. allgemein BROWN 1976). In der Regel sind jedoch, wie noch zu zeigen sein wird, für die Strukturierung natürlicher Kategorien beide Gesichtspunkte von Bedeutung.

Dieselbe Dichotomie gilt für den Bereich der wissenschaftlichen Kategorisierung, wie SIMPSON 1963: 3sq. am Beispiel der Zoologie demonstriert: die Exemplare einer bestimmten Species stehen untereinander in einer Ähnlichkeitsbeziehung, da sie alle gewisse morphologische Eigenschaften teilen; darüber hinaus stehen sie aber auch zueinander in der metonymischen Beziehung der reproduktiven Isolation, die sie von anderen Arten absondert. Dasselbe gilt für übergeordnete Taxa: ihre Mitglieder weisen untereinander nicht nur morphologische Ähnlichkeiten auf, sondem sind auch durch die Herkunft von einem gemeinsamen Vorfahren (also wiederum durch eine indirekte metonymische Beziehung) verbunden. Gerade an diesem Beispiel sieht man auch, daß die beiden Klassifikationsgesichtspunkte auch dort, wo sie zu (teilweise) identischen Ergebnissen führen, nicht miteinander verwechselt werden dürfen: ein Gegner der Evolutionstheorie wird ein System der Tier- und Pflanzenwelt ohne jeden Bezug auf die Phylogenese aufbauen; für einen anderen Forscher ist vielleicht der Autbau einer biologischen Systematik nur auf der Grundlage dieser metonymischen Beziehungen denkbar (dieser letzte Standpunkt wird etwa von Ax 1988 vertreten).

Es sei betont, daß in einer Taxinomie nicht nur die Bei-, sondern auch die Über- und Unterordnungsbeziehungen eine metaphorische Komponente aufweisen. Dies zeigt sich sehr schön in der Bedeutung, die Wendungen wie eine Art... (im Deutschen ohne von!) in vielen Sprachen erhalten: von taxinomischen Operatoren (Terminus nach GINZBURG/KREJDLIN 1982) entwickeln sie sich zu Heckenausdrücken (hedges), bei denen es nicht mehr um die Kategorienzugehörigkeit eines Gegenstandes, sondern um bloße Ähnlichkeit geht (zur englischen Konstruktion kind of cf. CRUSE 1986: 137sq., zu sort of HOMES 1988). ARISTOTELES hat die Verwandtschaft zwischen den horizontalen und den vertikalen taxinomischen Beziehungen klar erkannt und in seinem bekannten vierteiligen Schema ausgedrückt, wonach eine Metapher durch Übertragung von der Gattung auf die Art, von der Art auf die Gattung, zwischen zwei verschiedenen Arten derselben Gattung oder kata to analogon zustandekomme 
(Poetik 1457b, 6-9). Dieser Punkt ist bereits in der Antike mißverstanden worden: in der Aufzählung der Poetik sah man die bloße Zusammenfassung von heterogenen Erscheinungen und schlug die ersten beiden Metaphemtypen zur Synekdoche -- eine einseitige Entscheidung, die sich als erstaunlich zählebig erwies. ${ }^{8}$

Man hat bemerkt, daß "das reiche Netz von Beziehungen, die den inneren Aufbau von Partinomien ausmachen, in Taxinomien keine natürliche Entsprechung besitzt" (TVERSKY 1990: 341; meine Übersetzung). Tatsächlich wirkt im Vergleich zu den vielfältigen Formen, die allein die Teil-Ganze:-Beziehung annehmen kann (MILLER 1991: 164sq.), die Ähnlichkeitsbeziehung recht eindimensional. Dieses Verhältnis kehrt sich jedoch für die Kategorienmitglieder selbst um: während in Partinomien jeder Teil auf jene Seite seines Begriffsinhaltes reduziert wird, die seine Zugehörigkeit zum Ganzen begründet, erlauben Taxinomien durch vertikale und horizontale Gegenüberstellung ihrer Mitglieder die Aktivierung sehr unterschiedlicher Vergleichsdimensionen; die Merkmalsanalyse ist deshalb das natürliche Korrelat der taxinomischen Kategorisierung. Zur weiteren Strukturierung von Taxinomien trägt der asymmetrische Charakter von Ähnlichkeitsbeziehungen bei (TVERSKY 1977).

Nicht nur das Ergebnis, sondern auch der Prozeß der Kategorisierung selbst ist vom Gegensatz zwischen Kontiguität und Ähnlichkeit geprägt. Je nachdem, ob dieser Prozeß vorwiegend von metonymischen oder von metaphorischen Beziehungen gesteuert wird, kann man von einem konkreten, situativen bzw. einem abstrakten, kategorialen Verhalten sprechen: bei ersterem werden die Gegenstände (bzw. Begriffe) nach ihrer gemeinsamen Zugehörigkeit zu einer bestimmten Situation, bei letzterem nach ihrer Zugehörigkeit zu einem taxinomischen Oberbegriff zusammengestellt. Ein Beispiel für die konkrete (situative) Kategorisierungsstrategie wäre die $\mathrm{Zu}$ sammenstellung von Messer, Glas, Salzfaß, Brotkorb auf Grund der Tatsache, daß all diese Gegenstände zu einem gedeckten Tisch dazugehören; ein Beispiel für die abstrakte (kategoriale) die Zusammenfassung von Beil, Säge, Spaten, Nadel, Dosenöffner zu einer Kategorie, die durch ein abstraktes funktionales Merkmal (Werkzeuge) zusammengehalten wird, ohne daß die einzelnen Gegenstände je gemeinsam Verwendung finden würden. ${ }^{9}$ Ein wesentlicher Unterschied zwischen den beiden

${ }^{8}$ In einer neueren russischen Untersuchung zur Metapher findet man sie bereits auf der ersten Seite: SKLJAREvSKAJA 1993: 5, ähnlich ZIOMEK 1990: 162 und ZYMNER 1993: 5, Anm. 9; cf. aber die GENERIC IS SPECIFIC Metaphor bei LAKOFF/TURNER 1989: 162-166.

${ }^{9}$ Die Termini abstrakt und konkret stammen von K. GolDSTEIN, die Termini kategorial und situativ von L.S. VYGOTSKIJ und A.R. LURIJA. Siehe GOLDSTEIN/SCHEERER 1941: 81-109 (cf. GELB/GOLDSTEIN 1925, WEIGL 1927) sowie LURIJA 1974: 58-105 und die differenziertere Darstellung des Denkens in Komplexen bei VYGOTSKIJ 1982: 118-184. Das Bild des Bastelns (bricolage) eignet sich zur 
Verhaltenstypen besteht darin, daß die abstrakte (kategoriale) Einstellung die konkrete (situative) einschließt, aber nicht umgekehrt: wer in der Lage ist, eine Kategorisierung nach abstrakten Gesichtspunkten vorzunehmen, wird bei Bedarf auch eine konkret-situative liefern können; Personen, die konkret-situative Kategorisierungen bevorzugen, ist dagegen der Übergang zu den abstrakt-kategorialen verschlossen. ${ }^{10}$ Es ist oft behauptet worden, daß Mitglieder traditioneller (d.h. vormoderner) Gesellschaften zum konkret-situativen Verhalten neigen; Verallgemeinerungen dieser Art bieten freilich viele Angriffsflächen. Festzustehen scheint jedenfalls, daß die formale Schulbildung westlichen Typs die abstrakte, kategoriale Einstellung entscheidend fördert."

Unter dem Einfluß der formalen Schulbildung ist man versucht, dem abstrakt-kategorialen Vorgehen und den taxinomischen Hierarchien, zu denen es führt, den Status einer Norm zuzubilligen. R.M. FrUMKINA und ihre Mitarbeiter (A.V. MIXEEV, A.D. Mostovaja, N.A. RuUmina) haben in einer Reihe von Experimenten seit dem Anfang der 80er Jahre die Struktur natürlicher Kategorien wie ovošči i frukty (Obst und Gemüse), bljuda (Gerichte), posuda i kuxonnaja utvar (Küchengeschirr und Besteck) untersucht und dabei festgestellt, daß das Operieren mit konkret-situativen Assoziationen auch im Kategorisierungsverhalten moderner Großstadtbewohner einen viel größeren Raum einnimmt, als man ihm bisher zubilligen wollte; die Ergebnisse dieser Experimente sind in FrUmkina et al. 1991 und FrUmKINA/MIXEEV 1996 zusammengefaßt.

Die Methode, die R.M. Frumkina und ihre Mitarbeiter angewand haben, ist die des freien Klassifikationsexperimentes. Bei dieser Methode, die von G. A. MILLER vorgeschlagen wurde (MILLER 1969; MILLER 1971), wird den Versuchspersonen eine gewisse Anzahl (ca. 50) Kärtchen gegeben, auf denen die zu untersuchenden Wörter (ein Wort pro Kärtchen) stehen. Die Anweisung lautet, diese Kärtchen nach der Bedeutungsähnlichkeit der Wörter zu Stapeln zusammenzufassen. Anzahl und Umfang der Stapel (d.h. der Kategorien; im folgenden meist als Klassen bezeichnet) sind frei, eine Zeitbeschränkung wird nicht vorgenommen.

Das Ergebnis kann man für jede Versuchsperson in Form einer quadratischen Matrix darstellen. Jede Zeile und jede Spalte dieser Matrix entspricht einem der untersuchten

Illustration des konkret-situativen Kategorisierungsverhaltens besonders gut, doch läßt sein Gebrauch bei LĖVI-STRAUSS 1962: 26sqq. die nötige Klarheit vermissen.

${ }^{10} \mathrm{Zu}$ diesem wichtigen Punkt s. GoldSTEIN/SCHEERER 1941: Isqq., 83sqq.; LURIJA 1974: 68 und die Diskussion bei FrUMKINA/MIXEEV 1996: 36sq.

"Cf. neben den bereits zitierten Arbeiten HALLPIKE 1979: 169-235 (von BERLIN 1992: 135sq. scharf kritisien) und TULVISTE 1991. 
Wörter. Eine (1) an der Kreuzung einer Zeile mit einer Spalte bedeutet, daß die Versuchsperson die entsprechenden Wörter in dieselbe Klasse, eine (0), daß sie sie verschiedenen Klassen zugeordnet hat. Aus der Addition aller Einzelmatrizen ergibt sich eine Matrix, bei der an der Kreuzung zweier Wörter die Anzahl der Versuchspersonen steht, in deren Klassifikation diese Wörter Mitglieder einer Klasse sind. Zieht man diese Zahl von der Gesamtzahl der Versuchspersonen ab, so erhält man die Anzahl der Versuchspersonen, die die betreffenden Wörter in verschiedene Klassen eingeordnet haben. Es ist naheliegend, in dieser letzten Größe eine Operationalisierung des Begriffs der Bedeutungsvers:hiedenheit zu erblicken, die damit als Abstand zwischen den Punkten eines semantischen Raumes interpretiert wird, zumal dieser Abstand die Bedingungen einer Metrik erfüllt (MILLER 1969: 171sqq.).

Die Ergebnisse eines freien Klassifikationsexperimentes können erst dann Gültigkeit beanspruchen, wenn es gelingt, ihnen eine plausible semantische Interpretation zuzuordnen -- wenn wir, um es mit den Worten MILLERs selbst auszudrücken, "aus der Messung des Abstandes zwischen Begriffen etwas über die Koordinaten ihres Universums erfahren können" (MILLER 1971: 569; meine Übersetzung). Diese Koordinaten stellt sich MILLER als ein System von Merkmalen vor, die nach dem Prinzip der Klasseninklusion unter den Elementen des Wortschatzes eine taxinomisch-hierarchische Ordnung konstituieren. Der semantische Abstand zwischen zwei Wörtern hängt im Rahmen dieses Modells von ihrer Ähnlichkeit ab, die als Anzahl ihrer gemeinsamen Merkmale verstanden wird: glue, der Leim, und varnish, der Firnis, stehen semantisch einander deshalb besonders nahe, weil es sich beide Male um zähflüssige, klebrige Substanzen handelt, die an der Luft schnell trocknen. Als geeignetes Verfahren zur Aufdeckung der Merkmalshierarchie, die einem bestimmten Wortschatzausschnitt zugrundeliegt, wird die Clusteranalyse nach JOHNSON 1967 empfohlen. Nur am Rande wird auf die Möglichkeit hingewiesen, daß der Zusammenfassung zu einer Kategorie auch andere Kriterien zugrundeliegen könnten: wenn etwa jack, der Wagenheber, und wheel, das Rad, miteinander verbunden werden, so geschieht das sicher nicht, weil ihre Inhaltsseiten besonders viele Merkmale gemeinsam hätten, sondern einfach deshalb, weil man den Wagenheber braucht, wenn der Reifen geplatzt ist und das Rad ausgewechselt werden muß (MILLER 1969: 187).

Die Gültigkeit der Anschauung, daß der Wortschatz natürlicher Sprachen über weite Strecken hinweg taxinomisch-hierarchisch strukturiert ist, ist keine aus den Klassifikationsentscheidungen der Versuchspersonen gezogene Schlußfolgerung, sondern eine Voraussetzung, die der Anlage des Experimentes selbst zugrundeliegt: bereits die Auswahl der von Miller untersuchten Wörter legte, wie zu Recht bemängelt wurde (FrUMKINA/MIXEEV 1985: 69sq.), eine taxinomische Kategorisierung nahe. Wendet man dieselbe Methode auf die Untersuchung natürlicher Kategorien wie posuda i kuxonnaja utvar' (Küchengeschirr und Besteck) oder ovošči i frukty (Obst und Gemüse) an, so gelangt man, wie R.M. FrUMKINA und ihre Mitarbeiter gezeigt haben, zu ganz anderen Ergebnissen. 
Das erste, was an den Klassifikationen der Versuchspersonen von R.M. FRUMKINA auffällt, ist die Vielfalt der Einteilungskriterien, wobei die Zusammenstellung nach gemeinsamen Merkmalen (Material oder Form), d.h. nach der bloßen Ähnlichkeit, keineswegs eine beherrschende Stellung einnimmt. Mindestens ebenso häufig ist Bildung von Kategorien nach funktionalen oder situativen Gesichtspunkten, wobei zwischen diesen beiden Spielarten keine scharfe Trennlinie gezogen werden kann: vilka 'Gabel', ložka 'Löffel' und nož 'Messer', stupka 'Mörser' und pestik 'Stößel', caška 'Tasse' und bljudce 'Untertasse' sind sowohl durch ihre Funktion als auch durch ihr gemeinsames Vorkommen in bestimmten Alltagssituationen miteinander verbunden. Ganz deutlich zeigt sich das Vorgehen nach funktional-situativen (d.h. metonymischen) Assoziationen, wenn die Vpn ihre Entscheidungen mit Ausdrücken begründen wie to, čto svjazano s poxodom 'das, was mit einem Ausflug verbunden ist', servirovka prazdničnogo stola 'das, womit man eine Festtafel deckt', predmety dlja ¿arenija 'Gegenstände zum Braten'. Ähnliche Beobachtungen lassen sich -- wenn auch nicht so ausgeprägt wie in der Kategorie posuda i kuxonnaja utvar' (Küchengeschirr und Besteck) -- auch bei den Kategorien bljuda (Gerichte) bzw. ovošči $i$ frukty (Obst und Gemüse) machen (FrUMKINA et al. 1991: 69-83, 94sqq.).

Die Heterogenität der Einteilungskriterien entsteht nicht erst durch die Überlagerung der individuellen Klassifikationen, sondern ist bereits in ihnen angelegt: nur in den seltensten Fällen (10 von 150 ) bemühen sich die Vpn, ihre Klassifikation nach einem einheitlichen Schema durchzuführen. Der Wechsel des Gesichtspunktes beim Übergang von der einen Klasse zur anderen stellt nicht die Ausnahme, sondern die Regel dar. So hat ein zwanzigjähriger Student in der Kategorie posuda i kuxonnaja utvar' (Küchengeschirr und Besteck) folgende Klassen gebildet: 'Gegenstände, die für den Genuß alkoholischer Getränke bestimmt sind'; "scharfe", "gefährliche" Gegenstände'; 'Gegenstände, die einen Hohlraum besitzen; in sie kann man gießen, legen, hineinschöpfen'; 'Gegenstände, die eine eigene Gruppe bilden, da sie zu keiner anderen Gruppe passen'; 'Gegenstände mit flacher Form'; 'löcherige" Gegenstände' (FrUMKINA et al. 1991: 89). Wie die Begründungen anderer Versuchspersonen zeigen, kann die gleichzeitige Anwendung mehrerer Kriterien sogar bei der Bildung einer einzigen Kategorie zur Geltung kommen.

Zum Abschluß dieses Unterkapitels wollen wir die Heterogenität natürlicher Kategorisierungskriterien am Beispiel einer Kategorisierung illustrieren, die ihre Entstehung nicht experimentellen Bedingungen verdankt, sondern mitten aus dem russischen Alltag gegriffen ist. Im UNIVERMAG MOSKVA stand im Oktober 1993 neben der Rolltreppe im Erdgeschoß folgende Aufschrift: 
5 Melkooptovaja torgovlja

4 Tovary dlja mužčin

Obuv', kostjumy, pal'to, plašči, bel’e, noski, galantereja, verxnij trikotaž, parfjumerija, tovary Južnoj Korei, Ispanii, Kipra, "Xolding-centr"

3 Tovary dlja ženščin

tkani

Obuv', palto, plašči, parfjumerija, bluzki, jubki, plat’ja, zoloto, mexa,

2 Verxnij trikotaż

Kożgalantereja, noski, pugovicy, bel'e, postel'noe bel'e, parfjumerija, tjul', kanctovary, plat ja, jubki, bluzki

1 Zerkala, suveniry, galantereja, parfjumerija, časy, zonty, platki, posuda, mebel', bytovaja texnika

Diese Aufzählung spricht für sich. Wir verweisen nur auf das Vorhandensein einer Reihe von Kategorien mit mehr oder weniger deutlich ausgeprägter situativer Komponente (kanctovary, mebel', posuda, postelnoe bel'e, tovary dlja mužčin, tovary dlja ženščin) und die unbekümmerte Verbindung verschiedener Kategorisierungsgesichtspunkte, die sich im dauernden Wechsel des Einteilungskriteriums (Material, Form, Funktion, Ursprungsland) und der Abstraktionsebene äußert. So werden obuv ${ }^{\circ}$ und noski, bel'e und postel'noe bel'e, zerkala und mebel' nebeneinandergestellt. Interessant ist auch die Andeutung übergeordneter Kategorien durch die (pluralische) Nennung eines ihrer zentralen Mitglieder: mit noski sind natürlich člki und kolgotki mitgemeint (in den Ständen selbst werden sogar perčatki und varežki verkauft!), unter zoloto findet man auch Silberschmuck etc.

\subsection{Zum Material der vorliegenden Untersuchung}

Unsere bisherigen Ausführungen haben gezeigt, daß natürliche Kategorien ein hohes $\mathrm{Maß}$ an innerer Strukturiertheit aufweisen. Die Schwierigkeit, bei der Beschreibung der Semantik konkreter Substantive die sprachliche von der außersprachlichen (enzyklopädischen) Dimension zu trennen (Kapitel 1.1), rechtfertigt unsere Hinwendung zur psychologischen Forschung (Kapitel 1.2); sie wirft jedoch zugleich die Frage auf, auf welche Weise die psychologischen Befunde in die sprachwissenschaftliche Analyse integriert werden können. Wir haben uns in dieser Arbeit dafür entschieden, bei der Auswahl des Materials der Vielfalt natürlicher Kategorien 
Rechnung zu tragen, bei dessen Analyse jedoch von allen Unterschieden zwischen einzelnen Kategorien bzw. Kategorienmitgliedern abzusehen.

Tatsächlich sind die Kategorien, die in dieser Arbeit untersucht werden, in ihrem inneren Aufbau recht unterschiedlich:

- die Kategorie pticy (Vögel) ist eine taxinomisch-biologische Kategorie von natural kinds, die untereinander starke perzeptuelle Ähnlichkeiten aufweisen.

Der taxinomische Charakter dieser Kategorie wird bereits aus den den Wörterbucherklärungen im MAS deutlich: in der Regel bestehen sie aus einem Eingangsteil, den man als verkürzte taxinomische Prädikation auffassen kann (zum Begriff der taxinomischen Prädikation s. ARUTJUNOVA 1980: 197-201), und einem Hauptteil, der enzyklopädischen Charakter trägt und gewissermaßen beliebig erweiterbar ist, z.B.

IVOLGA 'Lesnaja pevčaja ptica otrjada vorob'inyx, $s$ opereniem želtogo i černogo ili zelenovatogo creta'.

- der Zusammenhalt der Kategorie posuda (Küchengeschirr und Besteck) wird in erster Linie durch konkret-situative Beziehungen zwischen den einzelnen Mitgliedern gewährleistet.

- die Kategorie sredstva peredvižnija (Fortbewegungsmittel) ist rein funktional bestimmt. An dieser Stelle sei angemerkt, daß sich hinter funktionaler Gemeinsamkeit sowohl eine metonymische als auch eine metaphorische Beziehung verbergen kann, je nachdem, ob die fraglichen Gegenstände ihre Funktion in einer bestimmten Situation gemeinsam erfüllen oder nicht. Im ersten Fall kann man von funktional-situativen, im zweiten von kategorial-funktionalen Begriffen sprechen; danach wäre die Kategorie posuda als funktional-situativ, die Kategorie sredstva peredviženija als kategorial-funktional zu bezeichnen.

- bei den Kategorien frukty (Obst) und ovošči (Gemüse) treten schließlich alle drei Gesichtspunkte -- der taxinomisch-biologische, der funktional-situative und der kategorial-funktionale -- gemeinsam auf. So haben etwa die Versuchspersonen von R.M. FRUMKINA die Zusammenfassung von arbuz und dynja sowohl durch perzeptuelle Ähnlichkeit ("bol'šoe, vkusnoe, sočnoe") als auch durch gemeinsamen Ursprung ("baxčevye rastenija") begründet; bei kartofel", morkov', svekla, rediska, red'ka, repa, brjukva hön man Begründungen wie "ogorodnye", "[rastut] pod zemlej", "vyrastajut $v$ tot że god posle togo, kak ix posadili", aber auch "s-edobnye ovošči ili korni gomogennoj i plotnoj konsistencii", bei baklažany und kabački die Begründung "vmeste edjatsja" etc. (Frumkina et al. 1991: 100sq.). 
Ein Produktionsexperiment, das wir im September und Oktober 1993 in Moskau durchgeführt haben (s.u.), brachte erwartungsgemäß in allen fünf Kategorien ein hohes $\mathrm{Ma} \beta$ an horizontaler Profilierung (Exemplar-Dominanz) zutage: Substantive wie vorobej, tarelka, avtobus, jabloko und kapusta fehlten auf fast keinem Fragebogen, Substantive wie kokos, penka, artišok, drezina, čerpak wurden nur von vereinzelten Versuchspersonen genannt. Keine experimentellen Daten besitzen wir dagegen über die Stellung unserer Kategorien auf der vertikalen Achse der Kategorisierung. Vier davon (posuda, sredstva peredviženija, ovošči, frukty) stehen mit Sicherheit oberhalb des basic level; bei der fünften (pticy) sind die Verhältnisse komplizierter -- wahrscheinlich sind sowohl ptica als auch einige seiner Hyponyme (vor allem Hausvögel) auf dem basic level anzusiedeln. Die Aufgabenstellung unserer Arbeit war mit der Einbeziehung von Kategorien unterhalb des basic level, die selber keine oder nur wenige monolexematische Unterkategorien besitzen, nicht zu vereinbaren.

Nun liegt gewiß die Frage nahe, ob solche Unterschiede zwischen den natürlichen Kategorien von Unterschieden im Wortbildungsverhalten der entsprechenden konkreten Substantive begleitet werden. Soll die Wortbildungsanalyse eine zuverlässige Grundlage für die Beantwortung dieser Frage abgeben, so muß sie selbst von allen Unterschieden zwischen den einzelnen Kategorien bzw. ihren Mitgliedern absehen. Es ist verführerisch, die Wortbildungsparadigmen der funktionalen Kategorie sredstva peredviženija nach anderen Prinzipien zu analysieren als die der biologischen Kategorie pticy oder bei der Aufstellung von Wortbildungskategorien Ableitungen zentraler Mitglieder wie jabloko und vorobej stärker zu gewichten; in diesem Fall wäre jedoch die Ausgangshypothese (die Korrelation zwischen Wortbildungs- und Kategorienstruktur) als Voraussetzung in die Wortbildungsanalyse selbst eingegangen und könnte nicht mehr mit ihrer Hilfe überprüft werden. Wir werden deshalb in den folgenden Kapiteln alle Wortbildungsparadigmen nach denselben Prinzipien behandeln und jede Berufung auf die in diesem Kapitel diskutierte quantitative und qualitative Variation im Bereich der natürlichen Kategorisierung vermeiden.

Bei der Bestimmung der Substantive, die in jede der fünf genannten Kategorien fallen, haben wir uns für ein experimentelles Verfahren entschieden. Es sei wiederholt, daß dieser Entscheidung im Rahmen der paradigmatischen Methode keine prinzipielle Bedeutung zukommt: der Gebrauch von Wörterbüchern, die Introspektion oder ein Zufallsprinzip wären vom Standpunkt der Methode aus gleich legitim -auch wenn die Annahme, den Wortbildungsparadigmen einer zufälligen Auswahl von Substantiven liege dasselbe abstrakte Wortbildungsparadigma zugrunde, extrem unplausibel sein dürfte. Für unsere Wahl waren allein praktische Gesichtspunkte ausschlaggebend: die Wörterbücher waren für unseren Zweck wenig geeignet, da sie in ihren Definitionen nur von taxinomischen Kategorien Gebrauch machen: kapusta wird nicht als ovošc̆, sondern als ogorodnoe rastenie, lošad nicht als sredstvo peredviženija, sondern als krupnoe neparnokopytnoe životnoe eingeordnet etc. (cf. 
oben zu ivolga sowie WIERZBICKA 1985: 261-263); und auf die Introspektion kann man sich nur als Muttersprachler verlassen.

Wir haben deshalb im September und Oktober 1993 in Moskau ein Produktionsexperiment durchgeführt, dessen Verlauf und Ergebnisse in Anhang 1 wiedergegeben sind, und werden in den folgenden Kapiteln jene Substantive, die von unseren Versuchspersonen genannt wurden, auf ihre Wortbildungseigenschaften hin untersuchen. Außer den Ergebnissen dieses Experimentes standen uns folgende Quellen zur Verfügung:

- zu den Kategorien ovošči, frukty und posuda die Listen, die R.M. FRUMKINA und ihre Mitarbeiter ihren Experimenten zugrundegelegt haben (zu deren Zusammenstellung s. FrUmKINA et al. 1991: 63sq.);

- wertvolles Material enthält auch die Abhandlung von V.N. MASKADYNJA, Normativnye dannye dlja 50 substantivnyx kategorij. Na materiale russkogo jazyka, Kalinin [Tver'] 1987 (MASKADYNJA 1987), die jedoch leider unveröffentlicht geblieben ist. Sie enthält die Ergebnisse eines breit angelegten Experimentes, das an der Staatlichen Universität von Kalinin durchgeführt wurde. Die Ergebnisse zu den Kategorien, die den hier untersuchten Kategorien am nächsten standen, wurden mit freundlicher Genehmigung der Verfasserin in Anhang 2 abgedruckt und werden bei unserer Wortbildungsanalyse von Fall zu Fall herangezogen. 


\section{VOM ABLEITUNGSPAAR ZUM WORTBILDUNGSPARADIGMA}

In diesem Kapitel wird der Versuch unternommen, die beiden grundlegenden $\mathrm{Be}$ griffe der Ableitungsbeziehung und des Wortbildungsparadigmas auf explizite Weise $\mathrm{zu}$ definieren. Unser Vorgehen wird dabei streng korpusorientiert/analytisch und reduktionistisch/atomistisch sein:

a) Wir gehen von der methodischen Fiktion eines geschlossenen Wortschatzes aus, der uns in Form einer endlichen Menge von Wörtern gegeben ist. Diese Menge bildet das Korpus, das es -- mit all seinen Lïcken und Inkonsequenzen -- zu beschreiben gilt. Anders ausgedrückt: nicht der Prozeß der Wortbildung, sondern sein Ergebnis, die Wortgebildetheit, ist Gegenstand der Untersuchung.'

b) Bei der Aufstellung von Ableitungsbeziehungen wird eine doppelte Reduktion vorgenommen:

b1) das Wortbildungssystem wird auf einzelne Wort(bildungs)paare reduziert: die Entscheidung darüber, ob in einem Wortpaar eine Ableitungsbeziehung besteht oder nicht, muß unabhängig von allen anderen Wortpaaren der betreffenden Sprache, d.h. unabhängig von den in dieser Sprache bestehenden Wortbildungstypen getroffen werden; diese sollen ja erst aus den einzelnen Ableitungspaaren mit Hilfe der paradigmatischen (oder, falls wir uns für diesen Weg entschieden hätten, der syntagmatischen) Methode erschlossen werden.

b2) die Ableitungsbeziehung wird auf ihre zwei Komponenten reduziert: die formale und die inhaltliche Beziehung. Dies bedeutet, daß die Beziehungen auf der Ausdrucksseite und die Beziehungen auf der Inhaltsseite der Wörter vollkommen unabhängig voneinander untersucht werden müssen.

Diese zweite Reduktion ist eine zwingende Folgerung aus dem bekannten Kriterium von VINOKUR, wonach zur Aufstellung einer Ableitungsbeziehung zwischen zwei Wörtern sowohl eine formale als auch eine inhaltliche Verwandtschaft zwischen ihnen erforderlich ist ${ }^{2}$ : wenn man Verwandtschaft zwischen den Inhaltsseiten zweier Wörter auf Grund der Verwandtschaft zwischen ihren Ausdrucksseiten postuliert

' Nach der bekannten Unterscheidung von DoKULIL 1968: 205sq. Daß die Wortbildungslehre ihren Gegenstand sowohl unter einem synthetischen [dynamischen, generativen, prozessualen] als auch unter einem analytischen [statischen, taxinomischen, entitativen] Gesichtspunkt untersuchen muß, ist auch sonst oft betont worden (BREKI.E/KASTOVSKY 1977, VAN MARLE 1985: 81sqq.).

2 Vinokur 1959; cf. ZWANEnburg 1984: 131sq. (ohne Kenntnis von VINOKUR). 
(oder umgekehrt), dann verliert das Kriterium von VINOKUR seine Schärfe. IGOR' MEL'ČUK hat die unabhängige Beschreibung der Inhalts- und der Ausdrucksseite der Sprache zu einem eigenen methodischen Grundsatz erhoben:

\section{Principle of Autonomous Description of Signifieds}

The claim that one signified is simpler than another must be always made and substantiated quite independently of any formal (morphological) relations between the corresponding signifiers.

Cf. die noch allgemeinere Formulierung an einer anderen Stelle:

The signified and the signifier of a linguistic sign are fairly autonomous; there is no logical necessity for their structures to be parallel (although in a large number, perhaps even in the majority, of cases, they are).

Therefore, the signified and the signifier of a linguistic sign should be investigated and described as independently as possible. ${ }^{3}$

Man sieht unschwer ein, daß diese Grundentscheidungen die Reichweite unserer Untersuchung auf entscheidende Weise einschränken:

$\mathrm{Zu}$ a): Der Begriff der Wortbildungsproduktivität kann im Rahmen des analytischen Zugangs, der den Wortschatz der Sprache als ein geschlossenes System betrachtet, allenfalls im nachhinein eingeführt werden.

Zu b1): Daß zwischen slux 'Gehör' und slušat' 'hören', nicht jedoch zwischen um 'Verstand' und dumat' 'denken' eine Ableitungsbeziehung besteht, kann nur auf dem Hintergrund unserer Kenntnis des russischen Wortbildungssystems als Ganzen entschieden werden, die zwar die Alternation $x / \bar{s}$, jedoch keine Präfigierung mit $d$ möglich erscheinen läßt; die isolierte Betrachtung der betreffenden Wortpaare hilft hier nicht weiter, da die formale und die inhaltliche Verwandtschaft zwischen um und dumat nicht weniger eng als die zwischen slux und slušat' sein dürfte.

$\mathrm{Zu}$ b2): Im sprachlichen Zeichen sind Form und Inhalt unlösbar miteinander verbunden; deshalb lassen sich weder das Kriterium von VINOKUR noch das von MELČUK proklamierte "Principle of Autonomous Description of Signifieds" auf die Dauer aufrechterhalten. MEL'ČUK selbst zieht beim Vergleich der Ausdrucksseiten auch Erwägungen über "morphologische Operationen" heran, die zur höheren formalen Komplexität eines Zeichens beitragen können: so soll der Plural Väter im Deut-

${ }^{3}$ MEL'ČUK 1995: 444 und 426; in den Erstveröffentlichungen der Aufsätze wird dieses Prinzip nicht so deutlich akzentuiert, cf. jedoch MELČUK 1969: 134sq. = MELČUK 1995: 456. 
schen im Vergleich zum Kernmorphem /Vater/ nicht nur semantisch, sondern auch formal komplexer sein, da er aus der Ausdrucksseite von /Vater/ plus der morphologischen Operation der Umlautung besteht (MEL'ĊUK 1995: 428). Offensichtlich kann die Richtung dieser morphologischen Operation nicht unabhängig von Erwägungen inhaltlicher Art bestimmt werden. Aber auch bei der Analyse der Inhaltsseiten und der damit verbundenen Bestimmung von Ableitungsbeziehungen erweisen sich die erwähnten Prinzipien immer wieder als revisionsbedürftig: eine solche Revision stellt z.B. RAMMELMEYERs Vorschlag dar, neben der eigenlichen, lexikalischen Bedeutung eines Wortes eine spezielle Wortbildungsbedeutung anzusetzen, die aus seiner Ausdrucksseite erschlossen wird (RAMMELMEYER 1983: 220sqq.; cf. ZEMSKAJA 1973: 73-76).

Welche Konsequenzen sind aus diesen Schwierigkeiten zu ziehen? Soll man die reduktionistische, korpusorientierte Wortbildungsanalyse zugunsten eines holistischen, an der Intuition eines idealen Sprachbenutzers ausgerichteten Verfahrens aufgeben? Es läßt sich leicht zeigen, daß die Anwendung der holistisch-intuitiven Methode die Wortbildungslehre ebenfalls in unauflösliche Aporien verstrickt:

$\mathrm{Zu}$ a): Den Ausgangspunkt der synthetischen Wortbildungslehre bildet nicht die Asymmetrie zwischen der Ausdrucks- und der Inhaltsseite des abgeleiteten Wortes, sondern der Unterschied zwischen produktiven und unproduktiven Wortbildungsmustern. Das Problem der Abgrenzung von Systematik und Idiomatik wird jedoch damit nicht umgangen, sondern ledigich vom Einzelwort auf den Gesamtwortschatz verschoben: die Wörter, die die Wortbildungsregeln erfüllen, d.h. jederzeit neu produziert werden können, weisen keine idiomatischen Komponenten auf -- wie denn auch produktive Formanten im Allgemeinen semantisch kohärenter sind als unproduktive (ARONOFF 1976: 38sq., DRESSLER 1986: 540); und die Wörter, die gegen die Wortbildungsregeln verstoßen, müssen ohnehin im Lexikon als fest reproduzierbare Einheiten gespeichert sein und könnten deshalb mit den voll idiomatischen (d.h. nichtabgeleiteten) Wörtern auf eine Stufe gestellt werden. Die synthetische Wortbildungslehre ist auf die methodische Fiktion angewiesen, daß zwischen diesen beiden Gruppen eine scharfe Trennlinie gezogen werden kann.

Zu b): Der holistische Zugang läuft Gefahr, die Grenze zwischen Wortbildung und Etymologie zu verwischen, und damit die Wortbildungslehre auf den Stand zurückzuwerfen, aus dem sie VINOKUR herausgeführt hat.

Die Lösung ist deshalb nicht in der einseitigen Anwendung der einen oder der anderen Methode, sondern in deren dialektischer Verbindung zu suchen; wie beim Gegensatz von paradigmatischer und syntagmatischer Wortbildungslehre besteht unser Ziel in dieser Arbeit lediglich darin, durch eine klare, konsequente Herausarbeitung der einen Alternative die Voraussetzung für eine solche Verbindung zu schaffen. 


\subsection{Das Korpus: ein Metawörterbuch}

Die Frage nach der Auswahl eines geeigneten Untersuchungskorpus zerfällt in zwei Teilfragen:

a) Es sind die konkreten Substantive zu bestimmen, die zu einer der fünf untersuchten Kategorien gehören. Die Gesichtspunkte, die dabei zu berücksichtigen sind, und die Quellen, die uns zur Verfügung stehen, haben wir bereits in der Einleitung bzw. im ersten Kapitel vorgestellt;

b) Es sind jene Wörter auszuwählen, die als Ableitungen zu einem konkreten Substantiv in Frage kommen: es ist, mit anderen Worten, die Wortmenge zu bestimmen, aus der wir unsere Wortbildungsparadigmen zusammenstellen wollen. Diese zweite Frage soll in diesem Unterkapitel beantwortet werden.

Wie in der Einleitung bereits angedeutet, wollen wir in unserer Untersuchung der desubstantivischen Wortbildung nicht bei der Betrachtung einzelner Wortbildungsmittel ansetzen, sondern bei den semantischen Oppositionen, die innerhalb einer Wortfamilie auftreten. (Strenggenommen ist nicht die Wortfamilie als Ganzes, sondern nur das Wortbildungsparadigma unser Ausgangspunkt. Die genaue Definition des Wortbildungsparadigmas wird am Anfang des nächsten Unterkapitels geliefert.) Je mehr Ableitungen eine Wortfamilie enthält, desto mehr Oppositionen lassen sich mit ihnen bilden, und desto breiter ist die Basis, auf der unsere Analyse beruht. Wir sind deshalb darauf angewiesen, in unserem Korpus möglichst viele Wortfamilien mit mehr als nur einem oder zwei Mitgliedern aufzufinden. Da die Wortbildungsproduktivität substantivischer Wortstämme ohnehin hinter der Wortbildungsproduktivität verbaler (und vielleicht auch adjektivischer) Wortstämme deutlich zurückbleibt, erweisen sich selbst so umfangreiche Wortschatzkorpora wie ZAL (ca. 100000 Wörter) oder MAS (über 80000 Wörter) für unsere Zwecke als zu klein. BASı ist zwar größer, jedoch in seinem Wortbestand bereits jetzt veraltet. Die zweite Auflage dieses Wörterbuches (BAS2), in der die Entwicklungen im Wortschatz der 60er, 70er und $80 \mathrm{er}$ Jahre berücksichtigt sind, ist noch im Erscheinen begriffen.

Es scheint überhaupt ratsam, einer Wortbildungsuntersuchung mehr als nur ein Wörterbuch zu Grunde zu legen. Hier kommt uns der 1991 erschienene Svodnyj slovar' sovremennoj russkoj leksiki (SSS) zu Hilfe. Es handelt sich hierbei um ein Wörterbuch, das den Wortschatz von 14 russischen Wörterbüchern dieses Jahrhunderts (30er bis 80er Jahre) zusammenführt und auf diese Weise zu über 170000 Einträgen kommt (s. die Einleitung von R.P. RoGoŻNIKOVA, p. 3). In der rechten Spalte des Wörterbuches werden alle Wörterbücher genannt, in denen das betreffende Wort belegt ist. Unter den Wörterbüchern, die für den SSS unter diesem Aspekt ausgewertet wurden, befinden sich alle größeren einsprachigen Wörterbücher, von OŻE bis BASI, zwei Neologismenwörterbücher, die 13. Auflage des Rechtschreibewörterbuchs von S.G. BARXUDAROV et al., das orthoepische Wörterbuch von R.I. AVANESOV, zwei Fremdwörterbücher, ein Fachwörterbuch aus dem Bereich der 
Technik (Politexničeskij slovar') und zwei enzyklopädische Wörterbücher: der Sovetskij enciklopedičeskij slovar und die Bol'šaja sovetskaja énciklopedija (s. die Liste auf p. 4). Bedenkt man die Schwierigkeiten, mit denen die Abgrenzung des sprachlichen vom enzyklopädischen und des naiven vom wissenschaftlichen Wissen im Bereich der konkreten Substantive zu kämpfen hat (Kapitel 1.1), so ist die Einbeziehung enzyklopädischer Nachschlagewerke im SSS zweifellos positiv zu bewerten. Weniger einfach liegen die Dinge beim Politexničeskij slovar, dessen Wortgut ja ausschließlich der Fachsprache angehört. Da gerade im Bereich der Wortbildung zwischen Fach- und Gemeinsprache beträchtliche Unterschiede bestehen ${ }^{4}$, scheint die Einbeziehung von eng fachsprachlichen Wörtern in die Korpusanalyse nicht gerade sinnvoll zu sein. Wir werden deshalb alle Wörter, die einzig und allein im Politexničeskij slovar' belegt sind, aus dem Korpus ausschließen.

Es sei allerdings nicht verschwiegen, daß sich die Auswahl des SSS als Materialkorpus in mehrfacher Hinsicht als problematisch erwiesen hat:

a) Der SSS weist einen hohen Grad an Heterogenität auf, da er ja sehr unterschiedliche, zum Teil auch gewiß überholte (UŠA, BAS1) lexikographische Quellen dokumentiert. Neben einem Wort wie repišče 'Rübenfeld', das bereits in PAW als veraltet gekennzeichnet ist, finden wir Neologismen der 60er und 80er Jahre wie veloturist, oblepixouboročnyj und kartofelepečka; neben dem volkstümlichen višen'e oder orešen'e die Fachtermini ananasnye, orexovye, jablonevye etc. Diese Heterogenität ist die Kehrseite des lexikalischen Reichtums des SSS; sie wiegt vielleicht weniger schwer, wenn man bedenkt, daß die Annahme absoluter Homogenität bei jedem Korpus (wie auch bei jedem synchronischen Schnitt) eine Idealisierung bedeutet. (Das Adjektiv synchronisch kennzeichnet nicht den Untersuchungsgegenstand, sondern die Methode.) Aus den bereits oben dargelegten Gründen ist es im Fall der desubstantivischen Wortbildung ratsamer, bei dieser Idealisierung eher zu großzügig als zu restriktiv vorzugehen.

b) Eine Reihe weiterer Unzulänglichkeiten teilt der SSS mit den Wörterbüchern, die ihm zugrundeliegen. Dazu gehört in erster Linie die Tatsache, daß diese Wörterbüchern (allen voran BASl) veraltetes Wortgut mitschleppen, das jenseits der Grenzen der modernen russischen Standardsprache steht: "strannye slova, kotorye soveršenno neprivyčny i vosprinimajutsja kak to li nepravil'nye, to li neliteraturnye obrazovanija", wie es eine Muttersprachlerin nach Durchsicht dieser Arbeit ausdrückte. Andererseits erweist sich der SSS trotz seines Umfangs für eine Wort-

4 Die Formulierung bei BIRKEnMaIER/MOHL 1991: 57, wonach "die Bildung neuer Wörter [...] in den einzelnen Fachsprachen mit Hilfe der in der Gemeinsprache vorhandenen Wortbildungsverfahren" geschehe, greift unseres Erachtens zu kurz; man denke nur an die besondere Rolle, die lateinische und griechische Formanten im Bereich der Fachsprache spielen. 
bildungsuntersuchung immer wieder als unzureichend: gerade Ableitungen der gängigsten Wortbildungstypen werden in den Wörterbüchern meist nicht verzeichnet, da sich ihre Bedeutung dem Muttersprachler aus den Bestandteilen ohne weiteres erschließt. So findet sich etwa unter den Ableitungen der Substantive der Kategorie frukty (Obst) im SSS nur ein einziges Augmentativum (arbuzišče), obwohl solche Augmentativa auch von vielen anderen Kategoriemitgliedern gebildet werden können. Aber auch in einer anderen Hinsicht ist der SSS unvollständig: er verzeichnet nur Einzelwörter und keine Mehrwortbenennungen. Nicht selten treten jedoch in der Struktur der Wortfamilie gerade solche Mehrwortbenennungen als Zwischenglieder auf, z.B.

limon 'Zitrone' $\rightarrow$ limonnaja kislota 'Zitronensäure' $\rightarrow$ limonnokislyj 'Zitronensäure-' (cf. 'otnosjaščijsja k limonnoj kislote, soderžaščij limonnuju kislotu', BASI);

ščavel' 'Sauerampfer' $\rightarrow$ ščavelevaja kislota 'Oxalsäure' $\rightarrow$ šcavelevokislyj 'Oxalsäure-' (cf. 'v nazvanijax ximičeskix veščestv, imejuščix $v$ svoem sostave ščavelevuju kislotu', ibid.);

kon' 'Pferd' $\rightarrow$ konnaja gvardija 'Kavalleriegarde' $\rightarrow$ konnogvardeec 'der Gardist zu Pferde', konnogvardejskij 'von der Kavalleriegarde' (beides PAW).

Die methodische Ausrichtung unserer Arbeit verbietet es, solche Mängel durch gezielte Ergänzungen zu beheben: wir würden dann nicht mehr das Wortbildungssystem aus dem Korpus, sondern umgekehrt das Korpus aus dem Wortbildungssystem ableiten. Wir haben uns mit ihnen abzufinden -- zumal sie in jedem anderen russischen Wörterbuch als dem SSS in noch krasserer Form auftreten würden; ob sich durch die Benutzung eines Textkorpus größere Vollständigkeit und Homogenität der Wortbildungsparadigmen erreichen ließe, müssen künftige Untersuchungen zeigen.

Ein wichtiges Hilfsmittel stellt für unsere Arbeit das Wortbildungswörterbuch von A.N. TIXONOV dar (TIX) -- auch wenn wir in unseren Entscheidungen über die Struktur einzelner Wortfamilien oft von den dort vorgeschlagenen Lösungen abweichen werden. Dieses Wörterbuch ist mit ca. 145000 Wörtern nicht so umfangreich wie der SSS, mit dem es die wichtigsten Quellen gemeinsam hat (s. TIX p. 8sq.). Allerdings zieht TIX zur Auffüllung von Wortbildungsketten gelegentlich das Wörterbuch von DAL' heran, dessen Wortschatz ganz gewiß außerhalb der Grenzen dessen liegt, was man noch als zeitgenössische russische Literatursprache bezeichnen kann. Manche Wörter bei TIX sind in keinem anderen Wörterbuch belegt, sondern entstammen der Kartothek des Wörterbuchautors. Auch wenn wir gerne glauben, daß alle Wörter, die bei TIX verzeichnet sind, aus russischen Texten stammen (der Versuchung, strukturelle Leerstellen in Wortfamilien durch eigene Ableitungen zu beseitigen, ist bereits DAL' erlegen; sie dürfte auch für TIXONOV recht stark gewesen 
sein, wie die Ausführungen auf p. 9 erraten lassen), können wir solche Wörter in unsere Analyse nicht einbeziehen: da wir nämlich bei ihnen weder über ausreichenden Kontext noch über eine Wörterbucherklärung verfügen, müßten wir ihre Bedeutung aus ihrer Wortbildungsstruktur erschließen, was der hier eingeschlagenen Untersuchungsrichtung zuwiderlaufen würde. Schließlich kommt es immer wieder vor, daß bei TIX einzelne Wörter, die in den herkömmlichen Wörterbüchern verzeichnet sind, übersehen wurden (so z.B. das Beziehungsadjektiv oblepixovyj zu oblepixa [cf. MAS], obrešetočnyj zu obrešetka [cf. ORF], zarešečivat zu zarešetit' [cf. u.a. MAS] etc.; es fehlt auch z.B. das Substantiv strekać). Aus diesen Gründen haben wir es vorgezogen, dieser Arbeit nicht TIX, sondern SSS zu Grunde zu legen.

Unser Ausgangsmaterial besteht demnach aus allen im SSS verzeichneten Wörtern, die nicht ausschließlich im Politexničeskij slovar' belegt sind. Werfen wir nun einen Blick auf die Seiten des SSS, so stellen wir eine beträchtliche Vielfalt in der typographischen Gestaltung der Lemmata fest: viele Lemmata enthalten unter dem fettgedruckten Stichwort weitere, normal gedruckte Einträge, die in manchen Fällen durchnumeriert werden (kosjačók 1. kosjačók, 2. kosjačók), in anderen nicht (kotirovat kotírovát', kotirovát); gelegentlich enthalten auch die fettgedruckten Hauptlemmata mehrere Wörter (kotelř̌ik, kotël čik); an anderen Stellen weisen sie eine Numerierung auf (1. kotóko, 2. kotóko); bei manchen Einträgen wie kotlo... deuten drei vor- oder nachgestellte Punkte die Unvollständigkeit des Stichwortes an. Wir kommen deshalb nicht umhin, unsere Analysekorpus mit Hilfe weiterer Einschränkungen genauer abzugrenzen. Für die Wörter, die aus dem SSS zur Aufnahme in das Analysekorpus ausgewählt werden, wollen wir in diesem Unterkapitel den Ausdruck Lexem verwenden.

Als erstes ist das Problem der (morphologischen) Wortvarianten wie kotlovyj und kotlovoj, sivoronka und sizovoronka etc. abzuhandeln. Über die Variativität der Ausdrucksseite eines Wortes existiert in der Russistik eine umfangreiche Literatur, die neben solchen Titeln wie VINOGRADOV 1944, SMIRNICKIJ 1954, AXMANOVA 1957: 192-233 auch eine eigene Monographie (RoGoŻNikOVA 1966) umfaßt. Wir wollen uns auf eine Diskussion dieser Problematik nicht einlassen, sondern lehnen uns, was diesen Punkt betrifft, ganz an die Entscheidungen des SSS an. Dieses Wörterbuch verfährt so, daß eine, manchmal auch mehrere Varianten (zu deren Auswahl siehe die Einleitung Bd. 1, p. 9) als normativnye bzw. osnovnye angesehen und durch Fettdruck hervorgehoben werden; alle anderen Varianten werden eingerückt unter der Hauptvariante (den Hauptvarianten) aufgeführt. Falls die alphabetische Reihenfolge dadurch verletzt wird, erhalten auch die Nebenvarianten eigene Lemmata, von denen jedoch lediglich auf die Grundform verwiesen wird; typographisch treten diese Nebenlemmata in zweierlei Gestalt auf: a) ohne Fettdruck, eingerückt und von einem Gedankenstrich eingeleitet oder b) numeriert und fettgedruckt (dieser letzte Fall tritt dann auf, wenn die betreffende Wortvariante mit der Variante eines anderen Wortes zusammenfällt): 
kostroplita, kostraplita

kočedyk

kočadyk, kočadyg, kočetyk

katkij

kotkij

-- kotkij sm. katkij

-- kočetyk sm. kočedyk

2. garnitura $s m$. 1. garnitur

(Man beachte, daß die morphologischen Wortvarianten im Lemma nicht durchnumeriert werden.)

Als Fälle von Variativität nennt der SSS (Bd. 1, p. 9) Akzentschwankungen, "phonematische Varianten" und Genusunterschiede. Den ersten und den dritten Punkt können wir ohne weiteres übernehmen; der zweite ist nicht eindeutig -- die Beispiele, die dazu in der Einleitung des Wörterbuches aufgelistet werden, sind: mameljük, mamljük; zavódskij, zavodskój; udègéjskij, udèxéjskij; pézo, péso. Sie unterscheiden sich alle nur in einem Buchstaben (im zweiten Beispicl geht dieser Unterschied mit einem Akzentwechsel einher); im Wörterbuch selbst werden auch Fälle größerer Abweichungen als Wortvarianten eingestuft (man denke an das bereits aufgeführte sizovoronka/sivoronka). Man könnte deshalb versucht sein, diesen zweiten Punkt ganz auszuschließen und sizovoronka, sivoronka etc. als eigene Lexeme (d.h. letzten Endes auch als eigene Ableitungen von vorona) anzusehen -- wie z.B. čaševidnyj und čašeobraznyj zwei synonyme, jedoch verschiedene Ableitungen von čaša sind. Dies hätte die unangenehme Folge, daß manche Wortfamilien durch semantisch identische und morphologisch einander sehr ähnliche Ableitungen aufgebläht würden; da es sich dabei überdies oft um weniger gebräuchliche Wörter handelt, würde die Gestalt der Wortbildungsparadigmen durch ein solches Vorgehen unnötigerweise entstellt. Wir werden deshalb an diesem Punkt den (intuitiv meist einleuchtenden, nicht selten allerdings auch widersprüchlichen ${ }^{5}$ ) Regelungen des SSS folgen, hinter denen ja nichts anderes als die lexikographische Praxis der gebräuchlichsten russi-

${ }^{5}$ So erscheinen etwa kuličenok und kuličonok als zwei selbständige Lemmata im SSS, da BASI nur die erste, ORF dagegen nur die zweite Form dieses Wortes verzeichnet. Zu galčonok, skvorčonok, syčonok liefern die Wörterbücher überhaupt keine Variante; im Lemma von gračonok finden wir wieder die Variante gračenok, die aus BAS1 übernommen wurde. 
schen Wörterbücher steht, und nicht nur kotlovyj und kotlovoj, sondern auch Fälle wie sivoronka und sizovoronka zu einem Lexem vereinigen.

Damit umfaßt unser Korpus alle fettgedruckten Lemmata, von denen nicht lediglich (wie im Fall von 2. garnitura) auf ein anderes Lemma verwiesen wird. Wo ein Lemma mehrere fettgedruckte Wörter enthält (d.h. wo die Autoren des SSS einem Wort mehrere osnovnye varianty zuordnen), werden diese zu einem Lexem zusammengefaßt; dasselbe gilt für die Varianten, die ohne Numerierung und Fettdruck unter dem Hauptlemma aufgeführt werden. Nebenlemmata (d.h. eingerückte Lemmata ohne Fettdruck) bleiben unberücksichtigt.

Ein weiterer Punkt, der behandelt werden muß, ist die Variativität des Wortes auf der Inhaltsseite, d.h. die Polysemie und ihre Abgrenzung von der Homonymie. Polysemie wird im SSS, der ja kein tolkovyj slovar' sein will, überhaupt nicht angezeigt; Homonyme werden unter einem (fettgedruckten) Lemma für jede einzelne Bedeutungsvariante (normal gedruckt) aufgeführt und mit einer fortlaufenden Nummer versehen:

sip

1. $\operatorname{sip} s m$ (siplyj zvuk)

2. $\operatorname{sip} s m$ (ptica)

Falls die Homonyme in keinem der benutzten Wörterbücher zusammen auftreten, erhält jedes von ihnen ein eigenes fettgedrucktes Lemma, hinter dem die Chiffren der Wörterbücher stehen, aus denen es stammt; diese Lemmata werden ebenfalls durchnumeriert:

1. bugi $s$ sr nskl (tanec) NS

2. bugi [ $s m]$ (narod) BSÉ SÉS

Wir werden in beiden Fällen der üblichen Praxis (und den Intentionen der Autoren des SSS) folgen und mehrere Lexeme ansetzen, auch wenn ihnen (wie im ersten Fall) im SSS lediglich eine fettgedruckte Form entspricht; das typographische Abgrenzungskriterium gegenüber den Wortvarianten, die ja ebenfalls keinen Fettdruck aufweisen, bildet die Numerierung. Selbstverständlich stimmen die russischen Wörterbücher in der Bewertung einzelner Homonyme oft nicht überein; wie diese Divergenzen in der rechten Spalte des SSS kodiert werden, wird im Vorwort, Bd. 1, p. 8 erklärt.

In unserem Zusammenhang ist die Behandlung der Wortbildungshomonymie in unserem Korpus von besonderem Interesse. Darunter sind jene Fälle zu verstehen, in denen mit ein und demselben Formanten mehrere nichtsynonyme Ableitungen gebildet werden: 
ostrjak

1. ostrjak (ostroumnyj čelovek)

2. ostrjak (ostryj konec čego-l.)

Nach der Einteilung bei TIXONOV/PARDAEV 1989: 49sqq. ist dies die zweite Kategorie von Wortbildungshomonymen; auf die genaue Definition der Wortbildungshomonymie kommen wir in 3.5.2 zurück. Man kann diese Art von Vielfalt in der Wortfamilie grundsätzlich auf zweierlei Art und Weise deuten: als semantische Erweiterung einer zunächst monosemen Ableitung oder als das Ergebnis der semantischen Vielfalt des Formanten; im zweiten Fall wäre zu entscheiden, ob der Formant als homonym, polysem oder einfach vage (nichtspezifisch) anzusehen ist. Wir können diese Fragen bei der Bestimmung unseres Korpus gar nicht anschneiden -strenggenommen wissen wir ja noch nicht einmal, ob ein Wort abgeleitet ist oder nicht. Da solche Wortpaare (oder Tripel oder Quadrupel etc.) immer gewisse durch die Ableitungsbasis bedingte nichttriviale Bedeutungskomponenten gemeinsam haben und wir sie eben noch nicht von den nichtabgeleiteten Wörtern unterscheiden können, müssen sie nach dem herkömmlichen Verständnis von Polysemie und Homonymie nicht als homonym, sondern als polysem angesehen werden. Der SSS, oder, um gerechter zu sein: die russischen Wörterbücher, die ihm zugrundeliegen, verfahren leider an diesem Punkte nicht einheitlich. Neben einem Beispiel wie kotel'ščik, dessen semantische Vielfalt ('1. Master po izgotovleniju kotlov (vo 2. znač.). 2. Specialist po obsluživaniju i èkspluatacii parovyx kotlov', MAS) nicht aufgeschlüsselt ist, erscheint lopatka, das mit seinen beiden Bedeutungen ('Umen's. k lopata; neboľsaja lopata. $[\ldots] 2$. Odna iz dvux ploskix širokix treugol'noj formy kostej $\checkmark$ verxnej casti spiny', ibid.) als (Wortbildungs)homonym behandelt wird. Diese Inkonsequenzen sind natürlich störend; wir werden in 2.2.4 eine spezielle Regelung treffen, um sie zumindest teilweise zu beseitigen. Fürs erste wollen wir sie jedoch dulden, d.h. zwei homonyme Lexeme lopatka, und lopatka, jedoch nur ein Lexem kotel'ščik ansetzen.

Im Falle der morphologischen Varianten und der (Wortbildungs)homonymie mußte eine ausdrückliche Festlegung auf jeden Fall getroffen werden. Etwas anders verhält es sich bei den nun folgenden zwei Einschränkungen, mit denen wir die Abgrenzung des Analysekorpus abschließen wollen.

Die erste Einschränkung betrifft Binomina wie kotel-akkumuljator, kotel-paroobrazovatel' oder kotel-utilizator, die im SSS als separate Stichwörter aufgeführt sind. Um unser Material nicht mit Formen aufzublähen, die nur am Rande zur morphologischen Wortbildung gehören, werden wir Binomina im folgenden aus der Betrachtung ausschließen, d.h. wie syntaktische Verbindungen der in ihnen enthaltenen Lexeme behandeln. Das Argument dafür liefert uns die Flektierbarkeit des Erstgliedes, die freilich im Einzelfall nicht eindeutig auszumachen ist, da der Sprachgebrauch zu schwanken scheint (cf. OżEGOV 1955). Dasselbe Kriterium trifft gewissermaßen auch 
für Bildungen wie poltarelki, polložki, polbutylki zu: zusammen mit MEL'ČUK 1985: 280-288 halten wir diese Fälle nicht für Ableitungen, sondern für Syntagmen, die aus dem Lexem pol und dem jeweiligen Substantiv bestehen. Das entscheidende Argument dafür bietet die Möglichkeit, das Vorderglied solcher Verbindungen vom Hinterglied durch ein Adjektiv zu trennen: pol zelenoj butylki. Polubutylka und polbutylka (cf. MEL'ČUK 1985: 288) stellen dagegen echte Ableitungen von butylka dar, wie die Tatsache zeigt, daß polu zelenaja butylka nur als [(polu zelenaja) butylka], nicht jedoch als [polu (zelenaja butylka)] verstanden werden kann. ${ }^{6}$

Der SSS führt nicht nur selbständige Wörter an, sondern auch deren Bestandteile, sofern ihnen in den ausgewerteten Wörterbüchern ein eigenes Lemma gewidmet ist: zakonno..., kotlo..., lit..., ...lit. Auch diese Elemente schließen wir aus der Betrachtung aus; deren Unvollständigkeit wird stets durch drei (vor- bzw. nachgestellte) Punkte angedeutet, so daß sie auch nach rein typographischen Kriterien problemlos abzugrenzen sind.

Die Aussonderung dieser beiden Kategorien von Stichwörtern des SSS geschieht vorwiegend nach praktischen Gesichtspunkten. Sowohl die Binomina (einschließlich der mit pol-präfigierten Bildungen) als auch Wortbestandteile wie kotlo... könnten ohne Schwierigkeiten in unserem Wortbildungsmodell untergebracht werden: die Binomina wären auf dieselbe Weise wie die Komposita zu behandeln (zu Gemeinsamkeiten zwischen Binomina und Komposita s. STALLMANN 1979), und Komponenten wie kotlo... oder avto... stehen den Beziehungsadjektiven sehr nahe, die ja ebenfalls keine vollwertigen Nominationen darstellen. (In avto... oder velo... hat man sogar eine neue Klasse von unflektierbaren Adjektiven sehen wollen, die im Russischen meist etwas mißverständlich als analitičeskie prilagatel'nye bezeichnet werden. S. dazu Panov 1971, PANov 1968, IV: 105-134 und ZemSKaja 1992: 54sqq.).

Wir geben zum Abschluß dieses Unterkapitels einen knappen Überblick über die Regeln, die der Abgrenzung unseres Korpus zugrundeliegen. Wie bereits erwähnt, stellt dieses Korpus eine endliche Menge von Lexemen dar:

a) Als Lexeme gelten all jene Wörter, die im SSS ein fettgedrucktes Lemma (Hauptlemma) besitzen, sofern von diesem Lemma nicht lediglich auf ein anderes Lemma desselben Wörterbuchs verwiesen wird.

Anm. 1: Manche Lemmata sind im SSS durchnumeriert; bei den betreffenden Wörtern handelt sich um Homonyme. Jedes der so numerierten Homonyme wird als ein selbständiges Lexem angesehen, wobei wiederum die Einschränkung

${ }^{6}$ Die diesen Ableitungen von MELČUK 1985: 285sq. attestierte Defektivität des Deklinationsparadigmas gilt zumindest für polbutlylka nicht. cf. den Beleg rjadom $s$ nim uže ležit vtoraja oporožnennaja polbutylka aus BASI. 
gilt, daß das Lemma des betreffenden Homonyms mehr als nur einen Verweis auf ein anderes Lemma des SSS enthalten muß.

Anm. 2: Manche Lemmata enthalten mehrere fettgedruckte Wörter nebeneinander, z.B. koteľ̌ik, kotërčik. Es handelt sich dabei um Wortvarianten, die $\mathrm{zu}$ ein und demselben Lexem zusammengefaßt werden müssen.

Anm. 3: Nebenlemmata (d.h. eingerückte Lemmata ohne Fettdruck) bleiben unberücksichtigt.

b) Aus der Menge der Lexeme werden ausgeschlossen:

1. Binomina, bei denen beide Bestandteile flektiert werden; ferner Bildungen wie polbutylki, bei denen das Erstglied vom Zweitglied durch ein Adjektiv getrennt werden kann.

2. Wortbestandteile ohne autonomen Status (was im SSS stets durch drei vor- oder nachgestellte Punkte angedeutet ist);

3. Wörter, die nur im Politexničeskij slovar' belegt sind.

c) In die Menge der Lexeme werden zusätzlich jene Wörter aufgenommen, die im SSS eingerückt, ohne Fettdruck, aber mit eigener Numerierung unter dem jeweiligen Stichwort aufgeführt wurden. Nach den Konventionen des SSS handelt es sich dabei um Homonyme, die nicht zu einem einzigen Lexem zusammengefaßt werden dürfen.

Als Beispiel betrachten wir einen Auszug aus der linken Spalte des SSS, der Ableitungen von rešeto und rešetka enthält:

rešétina $s \check{z}$

1. rešétit' $g l$ nsv (pribivat' rešetnik)

rešétit'

rešetit', rešètit'

2. rešétit'

1. rešetit' $g l$ nsv (izrešečivat')

rešétit'

rešetit, rešétit

2. rešetit'

rešëtka $s \check{z}$

rešëtka, rešótka

rešétnik

1. res̉étnik $s m$ (čast’ krovli) 


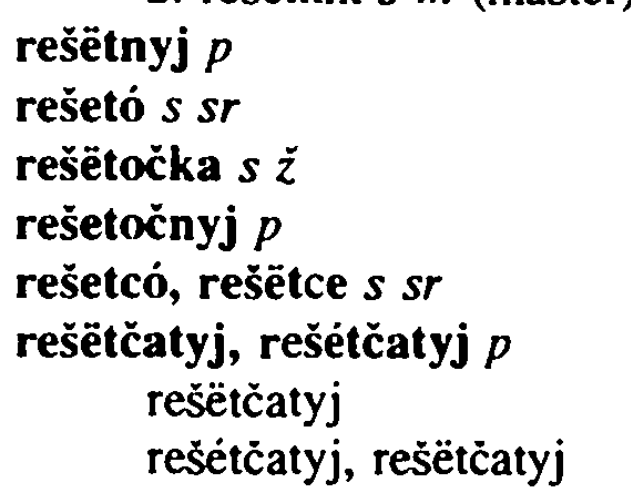

Die beiden vollständigen Lemmata von 2. rešétit' und 2. rešetit' lauten:

2. rešétit"

2. rešetit' sm. 1. rešetit'

sm. 1. rešétit'

Sie werden deshalb aus der Ausgangsliste gestrichen. Rešetit' und rešétit' (zu rešetit' 'pribivat' rešetnik'), rešetit' und rešétit' (zu rešetit' 'izrešečivat'), rešëtka und rešótka, rešetcó und rešëtce, rešëtčatyj und rešétčatyj sind morphologische Varianten ein und desselben Wortes; dagegen sind rešetit 'pribivat' rešetnik' und rešetit' 'izrešečivat" zwei verschiedene Wörter; dasselbe gilt für rešetnik 'čast' krovli' und rešetnik 'master'. Wir erhalten folgende Ausgangsliste (die Varianten stehen in eckigen Klammern):

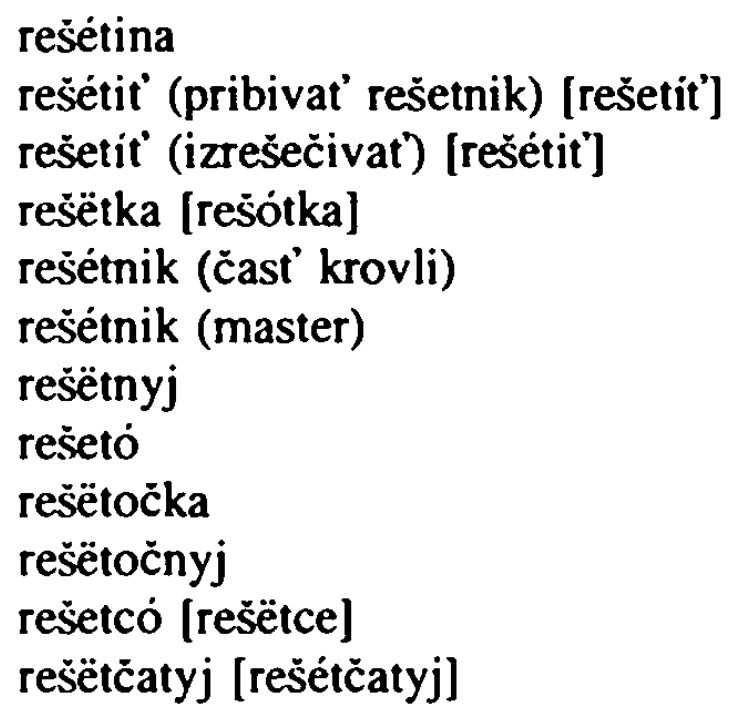

Das bei TIX verzeichnete rešëtčato-skladnoj fehlt im SSS und kommt deshalb für die weitere Wortbildungsanalyse nicht in Betracht. 


\subsection{Aufstellung der Wortbildungsparadigmen}

In diesem Unterkapitel wollen wir ein Regelsystem angeben, mit dem man auf eine möglichst eindeutige Weise das Wortbildungsparadigma eines konkreten Substantivs aus unserem Korpus bestimmen kann. Wir lehnen uns dabei an das Verfahren der Wortbildungsanalyse an, das TILMAN BERGER in seiner Dissertation Wortbildung und Akzent im Russischen (München 1986) vorgeschlagen hat; dessen zentraler Gedanke -- die gleichzeitige, jedoch getrennte Berücksichtigung der Inhalts- und der Ausdrucksseite der Wörter bei der Aufstellung von synchronen Ableitungsbeziehungen zwischen ihnen -- geht auf G. O. VINOKUR und I. A. MEL'ĊUK zurück (s. die Einleitung zu diesem Kapitel). Wir wollen den Beziehungen zwischen den Wörtern (Lexemen) unseres Korpus sowohl auf der Ausdrucksseite (formale Beziehungen) als auch auf der Inhaltsseite (inhaltliche Beziehungen) ein je eigenes Unterkapitel (2.2.1 und 2.2.2) widmen; der Rückgriff auf den dort eingeführten Begriffsapparat ermöglicht die Definition der Wortfamilie, die als Menge von Lexemen aufgefaßt wird (2.2.3). Im Rahmen der Wortfamilie wird nun die Lemmatisierung des SSS korrigiert, wodurch u.a. eine einheitliche Behandlung der Wortbildungshomonymie erreicht wird (2.2.4). Im letzten Unterkapitel (2.2.5) wird schließlich der Algorithmus erläutert, nach dem die Wortbildungsparadigmen der in dieser Arbeit untersuchten Substantive zu bestimmen sind.

\subsubsection{Formale Beziehungen zwischen Lexemen}

Entscheidende Impulse für die Untersuchung der Beziehungen zwischen den Ausdrucksseiten sprachlicher Zeichen gingen von MELČUK 1968 aus (eine überarbeitete Fassung dieses Aufsatzes liegt jetzt in MEL'CUK 1995: 425-441 vor). Die Übertragung des dort aufgestellten Modells auf die Wortbildung sieht sich jedoch vor folgendes Problem gestellt: MELČUK strebt eine Typologie der Beziehungen zwischen sprachlichen Zeichen an, die jeweils eine eindeutige Ausdrucks- und Inhaltsseite besitzen. Ein solches sprachliches Zeichen kann z.B. eine bestimmte (abstrakte) Wortform sein. ${ }^{7}$ Die Ableitungsheziehungen, die den Gegenstand der Wortbildung ausmachen, verbinden jedoch nicht einzelne Wortformen miteinander, sondern ganze Lexeme: nicht der Genitiv Singular gusenka ist vom Genitiv Singular gusja abgeleitet, sondern das Lexem gusenok vom Lexem gus'. Solchen Lexemen entsprechen bei flektierbaren Wortarten mehrere Wortformen mit z.T. voneinander stark abweichenden Ausdrucksseiten. Deshalb stellt sich die Frage, auf welche Weise diese Vielfalt

${ }^{7}$ Die Unterscheidung von konkreten Wortformen, abstrakten Wortformen und Lexemen setzen wir als gegeben voraus. Zu ihrer theoretischen Begründung s. KEMPGEN 1981. In den Fragen der Lemmatisierung, d.h. der Zusammenfassung von abstrakten Wortformen zu Lexemen, folgen wir dem SSS; einige Probleme, die sich im Bereich der Wortbildung aus der herkömmlichen Lemmatisierung ergeben, diskutien BERGER 1986: $121 \mathrm{sqq}$. Wir merken an, daß alle seine Beispiele (starsij, starejšij, opredelimyj, opredelennyj) im SSS als eigene Lemmata (Lexeme) erscheinen; cf. dort Bd. 1, p. 6sq. 
beim formalen Vergleich zweier Lexeme zu berücksichtigen ist: die formale Beziehung zwischen cyplenok 'Küken' und cypljatnik 'Hühnerhabicht' erscheint in einem ganz neuen Licht, wenn man statt des Nominativs Singular cyplenok den Genitiv Plural cypljat zugrundelegt.

Eine Explizierung des Begriffs der formalen Beziehung zwischen zwei Lexemen hat BERGER 1986 geliefert. Wir wollen BERGERs Modell zusammenfassend vorstellen, um anschließend einige Korrekturen und Ergänzungen daran anzubringen.

Als Ausdrucksseite der Wortformen sieht BERGER deren Schriftbild in der traditionellen kyrillischen Orthographie, d.h. keine Laut-, sondern eine Buchstabenfolge an. Diese Entscheidung erlaubt es, formale Beziehungen zwischen ihnen bzw. den Lexemen zu definieren, ohne auf die heikle Frage einer für das Russische geeigneten (morpho)phonologischen Transkription eingehen zu müssen.

Der entscheidende Schritt BERGERs besteht nun darin, neben der Grundform eines Lexems auch eine Basisform zu definieren. Die Grundform, die in etwa dem herkömmlichen Lexikoneintrag entspricht, dient in erster Linie dazu, auf das betreffende Lexem zu referieren; für die Beurteilung der formalen Beziehung zwischen zwei Lexemen ist die Basisform heranzuziehen (cf. p. 34sq.). BERGER gibt genaue Regeln an, nach denen sowohl die Grund- als auch die Basisform eines Lexems ermittelt werden kann; davon seien hier die Regeln zur Gewinnung der Basisform wiedergegeben, weil dieser Form auch in unserem Regelsystem eine Schlüsselrolle zukommt:

Die Basisform wird in normaler Orthographie geschrieben und endet mit einem Bindestrich. Sie trägt keinen Akzent. Im folgenden werden wir jeweils für die einzelnen Wortklassen festlegen, wie die Basisform gewonnen wird. [...]

1. Verben: Als Basisform wählen wir diejenige Buchstabenfolge, die in der 1. Ps. Sg. Prs. vor der Endung $-u$ bzw. $-j u$ steht, falls der Infinitiv auf $-t i,-\ddot{c}$ oder auf Konsonant $+-t^{\circ}$ endet. In allen anderen Fällen wählen wir die Buchstabenfolge, die im Infinitiv vor der Endung $-t$ ' steht. [...]
Beispiele:
nest $i$, nes'u...
Basisform: nes-
v'idet', v'ižu...
Basisform: vide-
umer'et', umr'u...
Basisform: umere-

Für die reflexiven Verben treffen wir die Konvention, daß wir das Postfix -sja nach dem Bindestrich in Klammern setzen.
Beispiele:
boj'at'sja, boj'us'...
Basisform: boja-(sja)
nest 'is', nes'us'...
Basisform: nes-(sja)

2. Adjektive: Als Basisform wählen wir diejenige Buchstabenfolge, die im Gen.Sg. mask. vor der Endung -ogo bzw. -ego steht. Hat das Adjektiv nur Kurzformen, so wählen wir die Form, die vor der Endung - $a$ der femininen Kurzform steht. 
Beispiele:

kras'ivyj, kras'ivogo...

$$
\text { r'ybij, r'yb'ego... }
$$$$
\text { d'olžen, dolžn'a... }
$$

Basisform: krasiv-

Basisform: $r y b$ -

Basisform: dolžn-

3. Substantive: Als Basisform wählen wir diejenige Buchstabenfolge, die im Gen. Sg. vor dem auslautenden Vokalbuchstaben steht. Falls kein Gen. Sg. existiert, wird dasselbe Verfahren mit dem Nom. Pl. durchgeführt. Bei den indeklinablen Substantiven, die keine grammatische Kategorie 'Kasus' aufweisen, wählen wir diejenige Buchstabenfolge, die vor dem auslautenden Vokalbuchstaben steht, wenn die einzige Wortform des Wortes auf Vokal endet, ansonsten ist die Basisform mit der einzigen Wortform identisch.

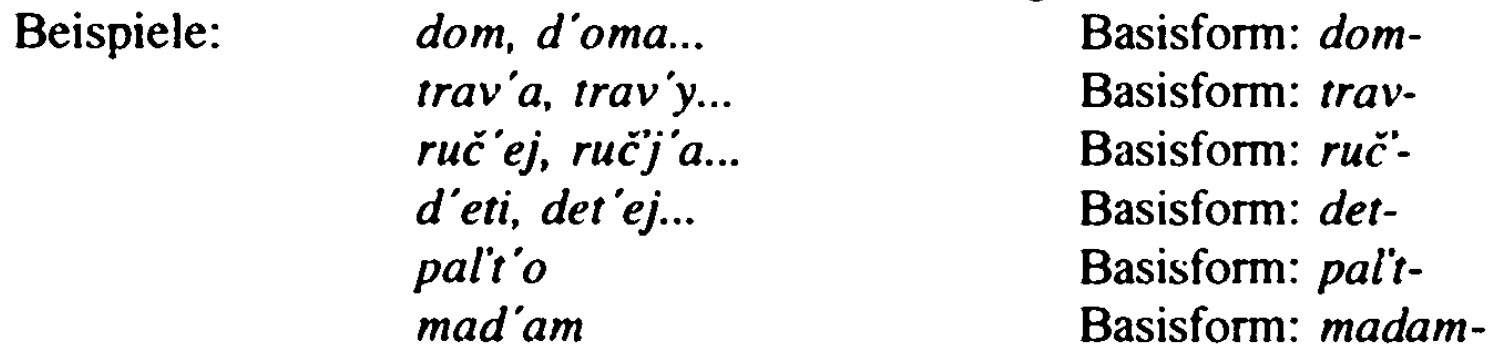

4. Zahlwörter und Pronomina: Wenn der Gen.Sg. (bzw. der Gen.Sg.mask., falls vorhanden) auf -ogo/-ego endet, wird wie bei den Adjektiven verfahren, ansonsten wie bei den Substantiven. Eine Schwierigkeit ergibt sich nur bei dem Wort on. Da der Gen.Sg. mask. eg'o lautet, ist die Basisform sozusagen 'leer'. Da das betreffende Wort aber für unsere weiteren Betrachtungen keine Rolle spielt, müssen wir keine besondere Regelung treffen.

Beispiele:
$k t o, k^{\prime}{ }^{\prime} o . .$.
Basisform: $k$ -
cej, $\ddot{c} \mathrm{eg}^{\circ} \mathrm{o} . .$.
Basisform: $\ddot{c}-$
pjat', pjat'i...
Basisform: pjat-
s'orok, sorok'a...
Basisform: sorok-
'oba, ob'oich...
Basisform: ob-

5. Unflektierbare Wörter: Hier ist die einzige Wortform zugleich Basisform (ohne das Akzentzeichen):

$\begin{array}{lll}\text { Beispiele: } & z \text { des } & \text { Basisform: zdes:- } \\ & z^{\prime} \text { avtra } & \text { Basisform: zavtra- } \\ & d a & \text { Basisform: da- }\end{array}$

(BERGER 1986: 128-131).

Für die Festlegung der formalen Beziehungen zwischen zwei Lexemen wird nun der Begriff der Varianten einer Basisform eingeführt und genau geregelt, welche Varianten von Basisformen zulässig sind und welche nicht. Diese Regelungen umfassen alle Varianten, die in der Flexion eines Wortes vorkommen (gusjat- ist also eine Variante der Basisform gusenk-), sowie die bekannten Erscheinungen der historischen Alternationen, des flüchtigen Vokals und der Stammverkürzungen (BERGER selbst meidet diese Termini): 
Eine Buchstabenfolge bezeichnen wir dann als Variante einer gegebenen Basisform, wenn eine der folgenden Bedingungen erfüllt ist:

a) Es gibt mindestens eine Wortform des durch die Basisform repräsentierten Wortes, in der diese Buchstabenfolge als Stamm auftritt.

b) Die fragliche Buchstabenfolge entsteht aus der Basisform oder aus einer Buchstabenfolge, die Bedingung a) erfüllt, durch eine der folgenden Operationen bzw. durch sukzessive Anwendung mehrerer solcher Operationen:

bl) durch Ersetzung des auslautenden Konsonanten durch einen anderen, wobei folgende Fälle zulässig sind:

$$
\begin{aligned}
& g, z d \leftarrow \dot{ } \\
& k, c, t \leftarrow \rightarrow \dot{c} \\
& t \leftarrow \hookrightarrow \check{s} \check{c} \\
& k \leftarrow \rightarrow c \\
& g \leftarrow \rightarrow z
\end{aligned}
$$

b2) durch Ersetzung der auslautenden Konsonantengruppe durch einen Konsonanten oder umgekehrt, wobei folgende Fälle zulässig sind:

$$
\begin{aligned}
& g, d \hookleftarrow z d \\
& \check{s} \check{c} \longleftrightarrow s t, s k \\
& p \leftrightarrow \rightarrow p l \\
& b \leftarrow \rightarrow b l \\
& m \hookleftarrow m l \\
& f \leftarrow \rightarrow f l \\
& v \leftarrow \rightarrow v l
\end{aligned}
$$

b3) durch Tilgung oder Hinzufügung eines Vokals vor dem letzten Konsonanten oder den beiden letzten Konsonanten

b4) durch Ersetzung eines Vokals innerhalb der zugrundegelegten Buchstabenfolge durch einen anderen Vokal.

c) Die fragliche Buchstabenfolge entsteht aus der Basisform oder aus einer Buchstabenfolge, die Bedingung a) erfüllt durch eine der folgenden Operationen:

cl) durch Tilgung des auslautenden Vokals

c2) durch Tilgung des auslautenden Konsonanten -n-

Beispiele (an erster Stelle steht die Basisform, an zweiter eine oder mehrere zulässige Varianten):

$\begin{array}{ll}\text { ljubi- } & \text { ljub- (a), ljubl- (a) } \\ \text { nosi- } & \text { nos- (a), noš- (a), naš (a, b4) } \\ \text { drug- } & \text { druz:- (a), druž- (b1) } \\ \text { knjaz- } & \text { knjag- (b1) } \\ \text { zeml- } & \text { zemel- (a), zem- (b2) } \\ \text { chodi- } & \text { chod- (a), chož- (a), chožd- (a, b2) } \\ \text { semg- } & \text { semuz- (b1, b3) } \\ \text { nes- } & \text { nos- (b4) } \\ \text { vydela- } & \text { vydel- (c1) }\end{array}$



pita- pišč- $(\mathrm{c} 1, \mathrm{~b} 1)$
buntova- buntu- (a), buntov- (c1)
bezdenežn- bezdenež- (c2) [von mir hinzugefügt, I.K.]

(BERGER 1986: 170sq.).

Auf dieser Grundlage werden schließlich drei Arten von formalen Beziehungen definiert:

a) Inklusionsbeziehung: Ein Wort $A$ ist formal in einem Wort $B$ 'enthalten', wenn die Basisform von A oder eine Variante dieser Basisform in der Basisform von $B$ enthalten ist;

b) Identitätsbeziehung: Zwischen zwei Wörtern $A$ und $B$ besteht eine Identitätsbeziehung, wenn $\mathrm{A}$ formal in $\mathrm{B}$ und $\mathrm{B}$ formal in $\mathrm{A}$ enthalten ist;

c) Intersektionsbeziehung: Zwischen zwei Wörtern A und B besteht eine Intersektionsbeziehung, wenn die Basisformen von $A$ und $B$ beide eine Buchstabenfolge $C$ umfassen, ohne da $B A$ in $B$ oder $B$ in $A$ formal enthalten ist (BERGER 1986: 172).

BERGER verwendet zur Illustration dieser Festlegungen Ableitungen aus dem Wortbildungsnest von buntovat; wir wollen jedoch hervorheben, daß formale Beziehungen auch zwischen Wörtern bestehen, zwischen denen überhaupt keine inhaltliche Beziehung vorliegt (wie auch umgekehrt inhaltliche Beziehungen keineswegs das Vorhandensein einer formalen Beziehung voraussetzen). Es besteht also etwa zwischen kniga und knjaz', mig und meždu eine Identitäts-, zwischen lob (Basisform lb-) und kolbasa eine Inklusions- und zwischen kaskada und laskovyj eine Intersektionsbeziehung. (Es sei auch kurz daran erinnert, daß die Akzentstelle bei der Ermittlung der formalen Beziehungen keine Rolle spielt -- die Basisform ist nicht akzentuiert; ferner gelten $e$ und $\ddot{e}$ als ein Buchstabe, so daß kotël-, die Variante der Basisform kotl-des Lexems kotël, z.B. in kotel'n-formal enthalten ist.)

Durch die Einführung der Basisform und ihrer Varianten gelingt es BERGER, bei der Beurteilung der formalen Beziehungen zwischen Lexemen die Variationsbreite ihrer Ausdrucksseiten zu berücksichtigen, ohne dabei die Genauigkeit der Analyse preiszugeben. Freilich sind die Regeln, nach denen die Varianten der Basisformen gewonnen werden, nach BERGERs eigenem Geständnis zu weit: "sie lassen noch wesentlich mehr Varianten zu, die aber für die Beschreibung der Wortbildung irrelevant sind, weil sie nicht tatsächlich belegt sind" (171sq.). Diese Bemerkung zeigt, daß jede Präzisierung des Begriffs der Varianten von Basisformen mit einer Preisgabe des Prinzips der getrennten Analyse von Inhalts- und Ausdrucksseite verbunden ist: welche Varianten tatsächlich belegt sind und welche nicht, kann man ja strenggenommen erst nach Bestimmung der Ableitungsbeziehungen feststellen, zu der die 
Ermittlung formaler Intersektions-, Identitäts- und Inklusionsbeziehungen zwischen den Lexemen die Voraussetzung bildet. Ein konsequent reduktionistisches Verfahren müßte auf den Begriff der Varianten von Basisformen ganz verzichten; ob dies praktisch durchführbar ist, darf man freilich bezweifeln.

Eine Reihe weiterer Einwände betreffen hingegen Punkte, an denen BERGERs Regelsystem durchaus verbesserungsfähig ist:

a) In der Regel bl) ist -- wahrscheinlich durch ein Versehen -- die Möglichkeit des Ersatzes eines auslautenden $s$ oder $x$ durch $\check{s}$ (und umgekehrt) ausgelassen worden, womit bei Ableitungspaaren wie gorox $\rightarrow$ gorošek, petux $\rightarrow$ petušinyj, uxo $\rightarrow$ ušanka, čerkes $\rightarrow$ čerkešenka, krasit $\rightarrow$ krašenie (man erinnere sich daran, daß das Partizip krašennyj, von dem man eine Variante der Basisform mit $\check{s}$ gewinnen könnte, nach Bergers Festlegung keine Wortform von krasit', sondern ein eigenes Wort ist und deshalb hier nicht herangezogen werden darf!) unnötige Schwierigkeiten entstehen. BERGER selbst leitet in einem späteren Kapitel veršok (mit verškovyj) von verx ab, bedient sich also der Möglichkeit, durch diese Alternation Varianten der Basisform zu bilden. (Verx und veršok darf man allerdings aus semantischen Gründen kaum als Ableitungspaar ansehen.)

b) Fatale Folgen hat das Fehlen einer expliziten Vereinbarung darüber, wie oft die Operationen zur Gewinnung der Varianten der Basisformen auf die Basisformen angewandt werden dürfen. BERGERS Formulierung legt, wenn man sie genau nimmt, folgendes nahe: auf die durch die Flexion gewonnenen Basisformen (Bedingung a) darf man eine oder mehrere der unter b) genannten Operationen oder aber (dieses oder ist exklusiv) höchstens ${ }^{8}$ eine der unter c) genannten Operationen anwenden. Diese Interpretation wird durch das von BERGER beigebrachte Beispiel pita $\rightarrow$ pišc̆, in dem zuerst $\mathrm{cl}$ ) (Tilgung des auslautenden Vokals) und danach bl) (Ersatz von $t$ durch $\check{s} \check{c}$ ) vorgenommen werden muß, widerlegt. Wenn man überhaupt keine Beschränkungen trifft, kommt man zu Varianten von Basisformen, neben welchen XLEBNIKOVs vnutrennee sklonenie als einfallsloses Seminaristenspiel erscheint: die Basisform von stena ist in šci enthalten, tarakan in turok, kaban in kobčik... Zu besonders kühnen Verwandlungen führt, wie man an unseren Beispielen sieht, die Möglichkeit, auslautendes $n$ zu tilgen -- von BERGER eingeführt, um gewisse Erscheinungen in der deadjektivischen Wortbildung (skudnyj und skudost', dušnyj und duxota) besser in den Griff zu bekommen (p. 169). Es ist unverständlich, warum dann diese Regel nicht auf Adjektive beschränkt wird, die ja als Kategorie bereits auf einem früheren Schritt der Wortbildungsuntersuchung (oder zumindest unabhängig von ihr) definiert werden können. Allerdings bliebe auch bei einer solchen Ein-

${ }^{8}$ Daß hier nicht mehr als eine Operation zulässig ist, muß man aus dem Fehlen einer expliziten Ermöglichung des Gegenteils schließen, wie sie unter b) gemacht worden ist. 
schränkung die lästige Folge, daß alle von Substantiven abgeleiteten Beziehungsadjektive auf $n$ zu diesen Substantiven in Identitätsbeziehung stehen, was die Ermittlung der Ableitungsrichtung (im engeren Sinne) durch das umständliche und nur unzureichend explizierte Verfahren der Seiten 180sqq. notwendig macht -- man wäre aber wenigstens von solch unangenehmen Nebeneffekten befreit wie die formale Inklusion von goršenja in allen Mitgliedern der Wortfamilie von goršok (das Ausgangswort eingeschlossen), von gusynja in den Ableitungen von gus' oder von kinut', dvinut', tronut in kidat', dvigat', trogat. ${ }^{\prime}$ Im Rahmen unserer Abhandlung kann auf die erwähnte Regel (c2) sehr wohl verzichtet werden. Außerdem legen wir fest, daß nicht mehr als zwei der auf p. 170sq. unter b1) bis c1) angeführten Operationen angewandt werden dürfen, um Varianten der Basisformen zu gewinnen, und daß dieselbe Regel nicht zweimal hintereinander angewandt werden darf (dabei soll nicht jede einzelne der unter b1) und b2) aufgeführten Operationen, sondern b1) und b2) zusammen -- neben b3), b4) und c1) -- als eine selbständige Regel gelten]. ${ }^{10}$

c) Noch eine weitere Einzelheit bedarf einer expliziten Festlegung: BERGER spricht von Vokalen und Konsonanten, übersieht dabei jedoch, daß er ja nicht mit lautlichen, sondern mit schriftlichen Einheiten operiert, auf die phonetische Termini nur bedingt angewandt werden dürfen: sollen das weiche Zeichen, das harte Zeichen und $i$ kratkoe als Vokale oder als Konsonanten gelten?" Ignorieren darf man das weiche Zeichen trotz vereinzelter Beispiele wie $z$ des ${ }^{\circ} \rightarrow$ zdešnij nicht, sonst besteht zwischen den Basisformen der Beziehungsadjektive auf $-i j$ und den Basisformen der entsprechenden Substantive eine (unerwünschte) Identitätsbeziehung, und die Basisform eines Adjektivs wie kuropačij (kuropač ${ }^{\circ}$ ) ist sogar in der Ableitungsbasis kuropatka enthalten. Wir setzen deshalb fest, daß die erwähnten Grapheme als Konsonanten zählen; wird zwischen dem weichen Zeichen und einem vorangehenden Konsonanten nach Regel b3) ein Vokal eingefügt, so wird das weiche Zeichen durch $j$ ersetzt, findet die Tilgung eines Vokals vor $j$ statt, so wird $j$ durch ' ersetzt, also:

${ }^{9}$ Der Vorteil, daß man durch die Tilgung von $-\boldsymbol{n}$ - das Wortbildungsverhältnis in stakan $\rightarrow$ stakašek, baran $\rightarrow$ barašek leichter in den Griff bekommt, fällt wegen der relativen Seltenheit dieser bereits von VINOKUR 1959 besprochenen Fälle kaum ins Gewicht.

${ }^{10}$ Sonst könnte man von kiosk-kiošč- und von diesem wieder kiot-ableiten (oder umgekehrt); oder es wäre (allerdings mit Anwendung dreier Umformungsregeln) gusenok in gusak enthalten (gusenk- $\rightarrow$ gusjat- [a] $\rightarrow$ gusat- [b3] $\rightarrow$ gusać[b1] $\rightarrow$ gusak-[b1]).

"In ZALIZNJAK 1967: 11 wird i kratkoe als Konsonant angesehen, das harte und das weiche Zeichen fallen in je eine eigene Kategorie. -- BERGERs Formulierung "auf einen Konsonanten (oder auf das 'weiche Zeichen' ')" auf p. 131 legt nahe, daß das weiche Zeichen nicht als Konsonant anzusehen ist. 
$r y b^{\circ}-\rightarrow$ rybij-, parodijn- $\rightarrow$ parod $n$ - (die generativen Potenzen, die unsere Regel im letzten Beispiel entfaltet, sind in manchen Fällen durchaus sinnvoll, cf. kopejk- $\rightarrow$ $k o p^{\prime} k$ - und die Basisform von kop'e, kop'-; im Wortpaar kop'e/kopejka ist natürlich die Zusammenfassung zu einer Wortfamilie durch semantische Erwägungen blockiert).

d) Schließlich tritt uns an dieser Stelle das bereits angeschnittene Problem der morphologischen Wortvarianten entgegen. Es führt dazu, daß ein Lexem mehrere Basisformen aufweisen kann. Für diesen Fall setzen wir fest, daß jede dieser Basisformen als Variante der anderen gilt (ohne daß dieser Ersatz den Status einer Regel wie a), b) oder c) hätte): um also zu entscheiden, daß noževoj/nožovyj in ponožovščina formal enthalten ist, kann man zunächst nožev-durch nožov-ersetzen und dann weiter vergleichen (theoretisch wäre nach diesem Ersatz noch die Anwendung zweier Regeln aus b) - c) zulässig; in unserem Fall brauchen keine weiteren Operationen vorgenommen zu werden). Bei den Verbalsubstantiven auf -ie, -'e sind sehr oft, jedoch nicht immer, Varianten auf - $e$, -ie zu verzeichnen. Die Auswahl der einen oder der anderen Form hat gewisse Folgen für die formalen Beziehungen innerhalb der Wortfamilie -- so ist z.B. wegen unseres Vorschlages, das weiche Zeichen als Konsonanten anzusehen, izrešečivanie in izrešečennyj, nicht jedoch podrešečen'e in podrešečennyj enthalten. Wir bestimmen deshalb, daß die Form auf $-e$ jeweils als Variante der Form auf -ie gilt (und umgekehrt), auch wenn nur die eine dieser beiden Formen im SSS verzeichnet ist.

Hier noch einmal die Zusammenfassung unserer Änderungs- bzw. Ergänzungsvorschläge zu den Bedingungen bei BERGER 1986: 170sq.:

a) Regel b1) wird um folgende Zeile ergänzt:

$$
s, x \leftarrow \dot{s}
$$

b) Regel c2) wird getilgt.

c) Das harte Zeichen, das weiche Zeichen und $i$ kratkoe gelten als Konsonanten. Wird vor einem weichen Zeichen ein Vokal nach Regel b3) eingefügt, so wird es durch $j$ ersetzt; wird vor einem $j$ nach dieser Regel ein Vokal getilgt, so wird es durch das weiche Zeichen ersetzt.

d) Zur Gewinnung der Variante einer Basisform dürfen höchstens zwei der unter bl) - cl) festgelegten Operationen angewandt werden, wobei es sich um zwei verschiedene Regeln handeln muß. Dabei werden alle unter b1) und b2) aufgeführten Operationen (neben b3, b4 und $\mathrm{cl}$ ) als eine einzige Regel angesehen. ${ }^{12}$

${ }^{12}$ Es darf also z.B. nicht bei der Gewinnung einer Variante zunächst st durch $\check{s} \check{c}$ (nach b2) und dann dieses $\check{s} \check{c}$ durch $t$ (nach bl) ersetzt werden. 
e) Falls ein Lexem mehrere Basisformen besitzt, so gilt jede von ihnen als Variante der übrigen. Bei deverbalen Substantiven ist unabhängig vom Zeugnis des SSS stets sowohl eine Basisform mit $i$ als auch eine mit weichem Zeichen anzusetzen (rešečeni/e]/rešečen'le]).

Diese Änderungsvorschläge sind auf unsere Bedürfnisse zugeschnitten und ganz gewiß nicht vollständig; bei einer Untersuchung der deverbalen Wortbildung wäre es z.B. wahrscheinlich notwendig, die Tilgung des Postfixes -sja aus den Basisformen der reflexiven Verben zuzulassen, da sonst z.B. razmax in razmaxat'sja, nicht jedoch razmaxat sja in razmax formal enthalten ist.

\subsubsection{Inhaltliche Beziehungen zwischen Lexemen}

Stellt man sich die Inhaltsseite eines Lexems als einen Komplex pragmatischer, semantischer und syntaktischer Eigenschaften vor (nach der bekannten Dreiteilung von C. W. MORRIS), so können die inhaltlichen Beziehungen zwischen Lexemen dreierlei Gestalt annehmen:

a) Die Lexeme unterscheiden sich in pragmatischer Hinsicht, wie z.B. rjabina 'Eberesche' und sein Deminutiv rjabinuška;

b) Die Lexeme unterscheiden sich in semantischer Hinsicht, wie z.B. fazan 'Fasan' und fazanina 'Fasanenfleisch';

c) Die Lexeme unterscheiden sich in syntaktischer Hinsicht, wie z.B. banan 'Banane' und bananovyj 'Bananen-'.

Wo sie in reiner Form auftreten, entsprechen diese drei Unterarten von Beziehungen der DokUl.lsschen Triade von Mutation, Modifikation und Transposition. ${ }^{13}$ Selbstverständlich können sich die Inhaltsseiten zweier Lexeme in zwei oder auch in allen drei Dimensionen voneinander unterscheiden. Bei den folgenden Ausführungen konzentrieren wir uns allerdings auf den semantischen Aspekt der inhaltlichen Beziehungen; auf die pragmatischen und die syntaktischen Aspekte der desubstantivischen Wortbildung werden wir in anderem Zusammenhang (Kap. 3.3 und 3.4) eingehen.

${ }^{13}$ Dokulil 1962: 46-49; DokUllL 1968: 209sq. Zur Mutation werden allerdings traditionellerweise auch viele Ableitungen gerechnet, die -- zumindest auf den ersten Blick -- keine pragmatische Komponente aufweisen (e.g. Kollektiva und Singulativa). 
Wir haben gesehen, daß zwischen den Ausdrucksseiten zweier Lexeme (ihren Basisformen) dreierlei formale Beziehungen bestehen können: Inklusions-, Intersektionsund Identitätsbeziehung. Legt man der Analyse der Inhaltsseite der Lexeme das Modell der Komponentenanalyse zugrunde, so können alle drei Beziehungsarten auch auf sie übertragen werden: die Inhaltsseite eines Lexems (sein Semem) stellt in diesem Fall eine (geordnete) Liste elementarer semantischer Merkmale (der Seme) dar ${ }^{14}$; zwischen zwei solchen Listen kann eine Inklusions- (das Semem A weist die gleiche Semkonstellation wie Semem B auf, das jedoch einige zusätzliche Seme enthält), eine Intersektions- (die Semkonstellationen von A und B überlappen sich) oder eine Identitätsbeziehung (Lexem $\mathrm{A}$ ist mit Lexem $\mathrm{B}$ synonym) bestehen.

Die Kombination der verschiedenen formalen und der inhaltlichen Beziehungsarten miteinander ergibt insgesamt neun verschiedene Arten von Beziehungen zwischen Lexemen, auf deren Grundlage man die Ableitungsbeziehung definieren kann; cf. das bekannte und mehrmals (etwa bei JACHNOW 1978: 11-17 oder LENNGREN 1978: 1724) übernommene Schema von MEL'ČUK 1968 (cf. MEL'ČUK 1967 und MEL'ĆUK 1969; alle drei Aufsätze sind jetzt in MELČUK 1995 nachgedruckt). Die eigentliche Crux besteht nicht in der Auszählung aller möglichen Fälle, sondern in der Zuordnung einzelner Lexeme zum einen oder anderen von ihnen, die ja theoretisch die vollständige Beschreibung des russischen Wortschatzes mit Hilfe elementarer semantischer Merkmale (mit Hilfe einer semantischen Metasprache) voraussetzt. Zum Glück ist die Wortbildungsforschung nicht auf die Realisierung solcher prinzipiell unabschließbaren Projekte angewiesen: sie hat es ja nicht mit primären Inhaltseinheiten der Sprache zu tun, sondern mit abgeleiteten; für den Nachweis einer inhaltlichen Beziehung zwischen gus und gusjatnik, banan und banannik, butylka und butylomoečnyj ist die vollständige semantische Analyse von gus; banan und butylka nicht notwendig -- es genügt die Möglichkeit, die Inhaltsseite des jeweils abgeleiteten Wortes durch einen Satz zu umschreiben, der das ableitende Wort enthält. Das bedeutet auch, daß wir für die Zwecke der Wortbildungsanalyse nicht auf eine semantische Metasprache zurückgreifen müssen, sondern das Russische als Metasprache seiner selbst verwenden können.

Unsere Definition der inhaltlichen Beziehung deckt sich deshalb mit der Definition der Motivationsbeziehung bei BERGER 1986: 165:

Wir sagen, daß zwischen zwei Wörtern eine 'Motivationsbeziehung' vorliegt, wenn die Bedeutung des einen von ihnen durch einen (russischen) Satz umschrieben werden kann, der das andere Wort enthält.

14 Der Einfachheit halber wird im folgenden stets von der Möglichkeit der Polysemie abgesehen. Auf die Konsequenzen, die die Polysemie der Basen für die Aufstellung der Wortbildungsparadigmen hat, gehen wir in Kap. 3.5.1 ein. 
Die dergestalt definierte inhaltliche Beziehung (oder Motivationsbeziehung) kann zwischen zwei Wörtern A und B in einer oder in beiden Richtungen bestehen. Falls A durch $B$, jedoch nicht $B$ durch $A$ umschrieben werden kann, liegt auf der semantischen Ebene eine (echte) Inklusionsbeziehung vor; falls dagegen sowohl A durch B als auch $B$ durch $A$ umschrieben werden kann, liegt entweder eine Identitäts- oder eine Intersektionsbeziehung vor. Semantische Intersektionsbeziehungen, bei denen eine Umschreibung in keiner der beiden Richtungen möglich ist, fallen nicht unter die gegebene Definition. -- Wie eine formale Beziehung zwischen zwei Lexemen keine inhaltliche voraussetzt, so setzt auch die inhaltliche Beziehung keine formale voraus, cf. die Wortpaare operacija und xirurg, glaz und videt, vrač und lečit etc.

Bei der Anwendung dieser Definition kann niemals jene Genauigkeit und Eindeutigkeit erreicht werden, die bei der Analyse der formalen Beziehungen zwischen den Lexemen (zumindest bei Heranziehung des Schriftbildes) möglich ist. Das Problem liegt in der Unterschiedlichkeit der Anforderungen, die man an die Bedeutungsumschreibungen der abgeleiteten Wörter stellen kann: zwischen der Wörterbucherklärung eines Wortes, die auf seine mögliche Wortbildungsstruktur keine Rücksicht zu nehmen braucht, und einer Umschreibung, die unter Preisgabe der Plausibilität ein Wort verwendet, dessen Ausdrucksseite in der Ausdrucksseite des Definiendums enthalten ist, besteht keine scharfe Grenze, sondern eine breite Übergangszone mit sehr vielen Zwischenstufen. Wir werden im folgenden bei der Bestimmung der Mitglieder einer Wortfamilie einen recht weiten, bei der Bestimmung des Wortbildungsparadigmas dagegen einen engeren Begriff von inhaltlicher Beziehung verwenden.

Die große Vielfalt der inhaltlichen Beziehungen zwischen ableitendem und abgeleitetem Wort hat die Wortbildungsforscher immer wieder zur Aufstellung von Typologien herausgefordert; s. ERMAKOVA/ZEMSKAJA 1991 und ULUXANOV 1992a, 1992b. Die Aufstellung allgemeinster Typen von inhaltlichen Beziehungen ("metaphorische", "hyponymische" Motivation etc.), die den Wortbildungskategorien auf ähnliche Weise übergeordnet wären wie die Wortbildungsarten (Suffigierung, Präfigierung etc.) den einzelnen Wortbildungsformanten, ist eine lohnende Aufgabe, der jedoch an dieser Stelle nicht weiter nachgegangen zu werden braucht. Nur auf einen Punkt wollen wir eingehen, weil er in den genannten Arbeiten nicht berücksichtigt wird und gerade im Bereich der konkreten Substantive von der allergrößten Bedeutung ist.

In Kap. 1.1 wurde auf die Unterschiede zwischen der Bedeutungsstruktur identifizierender und prädizierender Wörter hingewiesen (identificirujuščie vs. predikatnye slova, s. ARUTJUNOVA 1976: 326-356); identifizierende Wörter, wozu die natural kinds wie brusnika und aist, aber auch manche functional kinds gehören, haben, so wurde gesagt, eine extrem instabile sprachliche Inhaltsseite, indem sehr unterschiedliche Eigenschaften ihrer Denotate in die Liste ihrer definitorischen Merkmale aufge- 
nommen bzw. aus ihr wieder getilgt werden können. Wo sich eine dieser Eigenschaften in der Wortbildungsstruktur eines identifizierenden Wortes wiederspiegelt, bedeutet diese Instabilität seiner sprachlichen Inhaltsseite, daß es sehr leicht demotiviert (aber auch wieder remotiviert) werden kann. Die Wortbildungsstruktur hat deshalb die Tendenz, sogar in jenen Fällen erhalten zu bleiben, in denen die motivierende Eigenschaft gar nicht mehr in die Bedeutungsumschreibung des abgeleiteten Wortes aufgenommen werden kann, weil sie sich als sachlich falsch erwiesen hat. Wir geben dafür zwei Beispiele:

a) Die Vogelart der bananoedy 'Musophagidae' ernährt sich nach Ausweis der BSÉ keineswegs von Bananen: "pitajutsja [bananoedy] gl. obr. plodami, jagodami, semenami različnyx rastenij (nazv. "B." ne sootvetstvuet dejstvitel'nosti)", cf. "Nazvanie [bananoed] ne sootvetstvuet dejstvitel'nosti, t.k. bananami B[ananoedy] ne pitajutsja" (SËS). ${ }^{15}$

b) Zur Gerichtsbezeichnung jabločnik liefert BAS1 folgende Erklärung: 'zapekanka iz kartofelja s molokom i jajcami' und einen Beleg aus dem Jahr 1939: "[...] njan'ka nazyvaet [...] jabločnikom -- obyknovennuju kartofel'nuju zapekanku, ot kotoroj daže ne paxlo jablokom"16.

Wie diese Belege zeigen, ist die Beziehung zwischen bananoed und hanan bzw. jabločnik und jabloko für die Sprachbenutzer durchaus lebendig, auch wenn sie durch die Semantik des jeweils abgleleiteten Wortes nicht gedeckt wird. Es ist charakteristisch, daß identifizierende Wörter von Sprache zu Sprache sehr unterschiedliche Wortbildungsstrukturen aufweisen können: während der Lehrer auf russisch učitel' und der Weber $t k a c ̌$ heißt, sind die russichen Entsprechungen von pestruška, pestrjuga und pestrušnik, an denen sofort die formale Beziehung zu pestryj 'bunt' auffallt, 'Bachforelle', 'Rüsselstör' und 'Pfennigkraut'. Dies hängt damit zusammen, daß man sich einen Lehrer, der nicht lehrt (lehren kann), und einen Weber, der nicht webt (weben kann), ebensowenig vorstellen kann wie eine verheiratete Witwe; dagegen liegt im Begriff einer Forelle, der die schwarzen und rötlichen Flecken an den Seiten fehlen, kein logischer Widerspruch beschlossen. Anders ausgedrückt: eine Welt, in der die Forellen (etwa aufgrund von Umwelteinflüssen) ihre charakteristische Fär-

is Einen ähnlichen Fall stellt percejad dar. Cf. die Bemerkungen zu trjasoguzka bei ARUTJUNOVA 1980: 187.

${ }^{16}$ Von etymologischem Standpunkt aus wäre als Ableitungsmotiv wohl zemnoe jabloko heranzuziehen. 
bung eingebüßt haben, ist durchaus vorstellbar; eine Welt, in der die Eltern von Waisenkindern leben, ist es nicht. ${ }^{17}$

Am stabilsten scheint die Wortbildungsstruktur der identifizierenden Wörter dort zu sein, wo sie deren Stellung innerhalb einer Kategorie zum Ausdruck bringt; so ist sveklovica 'Zuckerrübe' ein Hyponym von svekla 'Rübe' -- ein Merkmal, das aus seiner Definition wohl kaum gestrichen werden kann. (Gänzlich unmöglich ist auch in diesèm Falle die Verschiebung der Grenze zwischen sprachlichem und enzyklopädischem Wissen freilich nicht, wie die Gedankenspiele PUTNAMs über die Hunde zeigen, die sich plötzlich nicht als Tiere, sondern als marsgesteuerte Automaten erweisen könnten.)

Diese Überlegungen legen nahe, zumindest bei den natural kinds für die Aufstellung inhaltlicher Beziehungen einen weniger strengen Maßstab als bei prädizierenden Wörtern anzulegen; es ist ja kaum ein Zufall, daß als Beispiele für die Schwierigkeiten des Ableitungsbegriffs immer wieder Tier- und Pflanzenbezeichnungen genannt werden -- so z.B. das notorische Wort zemljanika seit VINOKUR 1959: 422. ${ }^{18}$

\subsubsection{Zusammenfassung der Lexeme zu Wortfamilien}

Aufgrund ihrer formalen und inhaltlichen Beziehungen sollen nun die Lexeme zu Familien (in der Russistik: Wortbildungsnester, slovoobrazovatel'nye gnezda) zusammengefaßt werden. Den allgemeinen Leitfaden dieser Operation liefert das bereits mehrfach erwähnte Kriterium von ViNOKUR: zwei Wörter, zwischen denen sowohl eine inhaltliche als auch eine formale Beziehung (im oben definierten Sinne) besteht, sollen stets zu ein und derselben Wortfamilie gehören.

Im folgenden soll der Begriff der Wortfamilie mit Hilfe der Algebra der Relationen (nach ERNE 1982: 44-69) expliziert werden. Die mathematische Darstellungsweise hilft dabei, das Problem schärfer ins Auge zu fassen und gewisse unerwünschte Folgen, die sich aus der Anwendung des Kriteriums von Vinokur ergeben könnten, durch ergänzende Festlegungen auszuschließen.

Unseren Ausgangspunkt bildet das bereits abgegrenzte Materialkorpus, das man als eine (endliche) Menge von Lexemen auffassen kann. Wie BERGER 1986 wollen auch

17 Die Etymologie, die das Wort Forelle ebenfalls auf die Eigenschaft 'bunt, gesprenkelt' zurückführt, ist für unsere Argumentation an dieser Stelle belanglos.

${ }^{18}$ Cf. Rammelmeyer 1983: 220sqq. und Ermakova/Zemskaja 1991: $111 \mathrm{sq.}$ Mit den Beerenbezeichnungen des Englischen leitet auch ARONOFF 1976 seine Diskussion des Morphembegriffs ein (10sq.). 
wir die zusammengesetzten Lexeme aus dieser Ausgangsmenge ausschließen; die Notwendigkeit dieser Einschränkung wird im folgenden noch einsichtig werden. Die Definition der zusammengesetzten Lexeme lautet:

Ein Wort $\mathbf{A}$ ist dann 'zusammengesetzt', wenn es zwei Wörter $\mathbf{B}$ und $\mathbf{C}$ gibt, zwischen denen weder eine formale noch eine inhaltliche Beziehung besteht, und wenn zwischen $A$ und $B$ und zwischen $A$ und $C$ sowohl eine Motivationsbeziehung als auch eine formale Beziehung besteht. ${ }^{19}$

Die Definition der Wortfamilien stellt, wenn man die zusammengesetzten Wörter unberücksichtigt läßt, in mathematischem Sinne eine Partition (Zerlegung) der Menge $L$ aller nichtzusammengesetzten Wörter dar, d.h. ein Mengensystem $\Phi$ auf L mit den Eigenschaften
(1) $\varnothing \notin \Phi$
(2) $U \Phi=\mathrm{L}$
(3) Für alle $Y, Z \in \Phi$ gilt $Y=Z$ oder $Y \cap Z=\varnothing$ (ERNÉ 1982: 16).

Diese Festlegungen bedeuten, daß jedes Lexem einer Wortfamilie angehört (2), kein Lexem mehr als einer Wortfamilie angehört (3) (zusammengesetzte Wörter wurden ja ausgeschlossen!) und keine Wortfamilie leer bleibt (1).

Zwischen der Menge aller Partitionen von $L$ und der Menge aller Äquivalenzrelationen auf $\mathrm{L}$ existiert eine Bijektion (ERNE 1982: 51, FRIEDRICHSDORF/PRESTEL 1985: 16). D.h. jeder Partition von L kann genau eine Äquivalenzrelation auf L zugeordnet werden (und umgekehrt). Die eingangs gestellte Aufgabe kann also folgendermaßen umformuliert werden: man definiere die Äquivalenzrelation Q, die der Partition $\Phi$ der Menge $L$ in Wortfamilien entspricht, d.h. die Äquivalenzrelation, die der Beziehung "A gehört derselben Wortfamilie an wie B" entspricht.

Eine Äquivalenzrelation $\mathrm{Q}$ auf einer Menge $\mathrm{X}$ ist durch folgende Eigenschaften definiert:

(1) Reflexivität: $x Q x$

(2) Transitivität: aus $x Q y$ und $y Q z$ folgt $x Q z$

(3) Symmetrie: aus $x Q y$ folgt $y Q x$

19 Berger 1986: 174sq. Nur am Rande sei darauf hingewiesen, daß die Definition der zusammengesetzten Wörter bei BERGER (p. 174sq.) in Fällen wie učitel'sko-učeničeskij, razborno-sbornyj, očno-zaočnyj (alle Beispiele aus ZEMSKAJA 1991: 45) versagt. Glücklicherweise wird die Gültigkeit der Definition der Wortfamilie durch diesen Fehler nicht eingeschränkt. 
für alle $x, y, z \in X^{20}$.

Die der Äquivalenzrelation Q entsprechende Partition wird als

$$
\mu(Q):=\{Q y: y \in L\}
$$

definiert, wobei $Q y=\{x: x Q y \mid$ ist.

Bei der Zusammenfassung der Lexeme zu Familien sind die in den vorhergehenden Unterkapiteln behandelten Relationen auf $L$ z.u berücksichtigen: die allgemeine formale Beziehung $\mathrm{N}$ und die inhaltliche Beziehung (nach BERGER: Motivationsbeziehung) $M$. Sie sind beide trivialerweise reflexiv, jedoch nicht unbedingt symmetrisch: die formale Identität und die formale Intersektion sind symmetrisch, die (echte) formale Inklusion ist es jedoch nicht; und die inhaltliche Beziehung ist nur dann symmetrisch, wenn man sich entschließt, auch relativ abwegige Umschreibungen (gruša durch gruševidnyj und dgl.) als gültige Bedeutungsangaben zuzulassen. Die Symmetrie ist jedoch in beiden Fällen, wie der Gebrauch des Wortes "Beziehung" schon andeutet, relativ leicht herzustellen. Wir vereinbaren dazu einfach, daß mit $(A, B) \in N$ stets auch $(B, A) \in N$ und mit $(A, B) \in M$ auch $(B, A) \in M$ gilt.

Schwieriger steht es um die Frage der Transitivität: daß zwischen rom und promežutočnyj sowie zwischen promežutočnyj und żutkij eine formale Beziehung besteht, bedeutet natürlich nicht, daß eine solche auch zwischen rom und zuutkij anzusetzen wäre. Für die fehlende Transitivität inhaltlicher Beziehung können die Paare virus/mikroskop und mikroskop/steklo als Illustration dienen (cf. die Definitionen dieser Wörter in MAS und OŽE).

Für die Bildung der gesuchten Äquivalenzrelation $Q$ ist von der Schnittmenge $\mathrm{N} \cap \mathrm{M}$ auszugehen. Dies ist die Menge aller Paare, zwischen deren Mitgliedern sowohl eine formale als auch eine inhaltliche Beziehung besteht. Man sieht leicht, daß mit der Reflexivität und Symmetrie von $N$ und $M$ auch die von $N \cap M$ gegeben ist. Damit erfüllt $N \cap M$ die erste und die dritte Bedingung für eine Äquivalenzrelation. $N \cap M$ ist jedoch nicht transitiv.

Um die gesuchte Äquivalenzrelation $Q$ zu konstruieren, müssen wir also die Relation $\mathbf{N} \cap \mathbf{M}$ in geeigneter Weise erweitem. Die kleinste transitive Relation, die $\mathbf{N} \cap \mathbf{M}$ enthält -- die sogenannte transitive Hülle von $\mathrm{N} \cap \mathrm{M}$, in Zeichen: $(\mathrm{N} \cap \mathrm{M})^{\rightarrow}$-. erhalten wir durch folgendes Konstruktionsprinzip:

${ }^{20}$ Da eine Relation $Q$ auf $X$ letztlich nichts anderes als eine Untermenge des kartesischen Produktes $X \times X$ darstellt, sind die Schreibweisen $x Q y$ und $(x, y) \in Q$ äquivalent. 
$(N \cap M) \rightarrow:=\bigcup\left\{(N \cap M)^{n}: n \in\right\}$

(ERNE 1982: 56; zur Interpretation von $(\mathrm{N} \cap \mathrm{M})^{\mathrm{n}} \mathrm{s}$. ibid. pp. 10 und 46). Wie man unschwer beweisen kann, ist $(N \cap M) \rightarrow$ ebenfalls reflexiv und symmetrisch und damit eine Äquivalenzrelation $\mathrm{Q}$, die man der Zerlegung von $\mathrm{L}$ in Wortfamilien zugrundelegen kann.

Eine alternative Lösung würde darin bestehen, die Äquivalenzrelation, die die Mitglieder einer Wortfamilie miteinander verbindet und von allen anderen Lexemen trennt, nicht etwa als transitive Hülle der Schnittmenge von $\mathbf{N}$ und $\mathbf{M}(\mathbf{N} \cap \mathbf{M})^{\overrightarrow{ }}$, sondern als Schnittmenge der transitiven Hüllen von $N$ und $M\left(N^{\rightarrow} \cap M^{\rightarrow}\right)$ zu bestimmen, d.h. mit der Transitivität genauso zu verfahren, wie wir es bei der Symmetrie getan haben. In diesem Fall müßten jedoch alle indirekten formalen (bzw. inhaltlichen) Beziehungen zwischen Wörtern, die zufällig auch in einer inhaltlichen (bzw. formalen) Beziehung zueinander stehen, bei der Aufstellung der Wortfamilien mitberücksichtigt werden, was kaum sinnvoll erscheint.

In Worten ausgedrückt bedeutet die Bildung der transitiven Hülle folgendes: falls $A$ und $B$ derselben Wortfamilie angehören (d.h. falls zwischen ihnen sowohl eine formale als auch eine inhaltliche Beziehung besteht) und falls $B$ und $C$ derselben Wortfamilie angehören, so sollen auch $\mathrm{A}$ und $\mathrm{C}$ derselben Wortfamilie angehören (cf. BERGER 1986: 175). Tripel wie rom/promežutočnyj/žutkij oder virus/mikroskop/steklo werden nicht zu Wortfamilien zusammengefaßt, weil im allgemeinen mindestens bei einem der ihnen zugrundeliegenden Paare (in den Beispielen: bei beiden) die inhaltliche bzw. die formale Beziehung fehlt, so daß es erst gar nicht zu den Elementen von $\mathbf{N} \cap \mathrm{M}$ gerechnet wird.

Eine echte Schwierigkeit würde jedoch bei den zusammengesetzten Wörtern entstehen:

(steklo, stekloduv) $\in \mathrm{N}$, (steklo, stekloduv) $\in \mathrm{M}$

(stekloduv, dut') $\in \mathrm{N}$, (stekloduv, dut') $\in \mathrm{M}$

also auch

(steklo, stekloduv) $\in \mathrm{N} \cap \mathrm{M}$

(stekloduv, dut') $\in \mathrm{N} \cap \mathrm{M}$

und damit auch

(steklo, dut $) \in(\mathrm{N} \cap \mathrm{M})^{\rightarrow}$. 
Um dieses unerwünschte Ergebnis zu vermeiden, haben wir die zusammengesetzten Wörter zunächst aus der Zusammenstellung der Wortfamilien ausgeschlossen; síe können nun in sie wieder eingegliedert werden, indem man vereinbart, daß das zusammengesetzte Wort allen Wortfamilien angehört, die einen seiner Bestandteile enthalten. Ziel dieser Regelung ist es, der bereits von ROZWADOWSKI 1904 beobachteten funktionalen Äquivalenz von affixalen Ableitungen und Komposita (cf. sein Beispiel poln. wiatrak -- dt. Windmühle) durch deren gleichberechtigte Aufnahme in das Wortbildungsparadigma des jeweiligen Substantivs Rechnung zu tragen. Falls also zwischen einem Lexem A aus der Menge der nichtzusammengesetzten Lexeme und einem Lexem B aus der Menge der zusammengesetzten Lexeme sowohl eine inhaltliche Beziehung als auch eine allgemeine formale Beziehung besteht, so wird Lexem B in die Wortfamilie aufgenommen, der auch Lexem $A$ angehört. Die Zerlegung unserer Ausgangsmenge verliert hiermit die Eigenschaft der Disjunktivität, da sich die Wortfamilien im Falle der Komposita überschneiden. Im Rahmen unserer Fragestellung bleiben solche Überlappungen zwischen den Wortfamilien ohne Konsequenzen.

Bei der Auslegung der inhaltlichen Beziehungen, die erforderlich sind, um zwei Wörter zu ein und derselben Wortfamilie zusammenzufassen, wollen wir möglichst großzügig vorgehen. Die Bandbreite dieser Beziehungen reicht von der Identität der denotativen Bedeutung (lisa, lisica) bis zur gemeinsamen Zugehörigkeit zu einer übergeordneten Kategorie bzw. einer mehr oder weniger stark ausgeprägten Ähnlichkeit (čaša, čaška; bljudo, bljudce; pirog, pirožnoe). Die Forderung, daß das eine Wort mit Hilfe des anderen umschrieben werden kann, stellt ein bloßes diagnostisches Mittel dar; als solches ist sie nur dann brauchbar, wenn man von den so entstandenen Definitionen (Wortbildungsdefinitionen) weder sprachliche Natürlichkeit noch inhaltliche Genauigkeit verlangt. Die Definition muß möglich scheinen, aber nicht mehr: sie braucht kein gutes Russisch zu sein und darf bei Tier- und Pflanzenbezeichnungen (natural kinds) auch Merkmale enthalten, denen in den meisten Kontexten keine begriffskonstitutive Rolle zukommt und die man deshalb eher dem enzyklopädischen als dem sprachlichen Wissen über den betreffenden Gegenstand zuordnen möchte. Abgeleitete natural kinds, bei denen die Motivationsbeziehung weder intuitiv einleuchtet (wie etwa bei kuvšinka ${ }^{21}$ oder nožekljuv) noch aus den Wörterbuchdefinitionen bzw. dem betreffenden Artikel der BSE zu ersehen ist (wie etwa bei ložečnica ${ }^{22}$ ), werden mit ihren mutmaßlichen Basiswörtern nicht zu einer

"Kuvšinka wird aber in USA über čašečka definiert: 'vodjanoe rastenie iz semejstva kuvsinkovyx s okruglymi plavajuščimi list’jami i krupnymi belymi cvetami $v$ vide čašečki, nazyvaemoe takže beloj vodjanoj liliej', cf. kuvšin 'glinjanyj vysokij sosud, suživajuščijsja kverxu, s ručkoj'.

${ }^{22}$ Cf. BSĖ s.v.: "Lložečnica] arktičeskaja (Clochlearia] arctica) -- soćnoe rastenie $s$ belymi cvetkami i dlinnočereščatymi jajcevidnymi ili serdcevidnymi, kak 
Wortfamilie zusammengefaßt (also auch nicht als motiviert angesehen). Als Beispiele mögen die Lexeme sitovnik oder sitnik (das letztere in der Bedeutung 'lugovaja sornaja trava, po vnešnemu vidu napominajuščaja zlaki') dienen. Die Funktion abgeleiteter Benennungen von natural kinds besteht in erster Linie darin, das Gelingen der Referenz zu ermöglichen; ob sich der in ihnen ausgedrückte Begriffsinhalt mit den Eigenschaften des Denotats wirklich deckt oder nicht, fällt dagegen, wenn einmal der Gebrauch eine Benennung gefestigt hat, kaum ins Gewicht. ${ }^{23}$

Nicht nur die Definition der inhaltlichen, auch die Definition der formalen Beziehungen führt jedoch bei der Zusammenfassung von Lexemen zu Wortfamilien in eine Grauzone. Als problematisch erweist sich hier unsere (von BERGER übernommene) Festlegung, daß zur Zusammenfassung zweier Wörter zu einer Wortfamilie auf der formalen Seite nicht unbedingt eine Inklusions- bzw. Identitätsbeziehung erforderlich ist, sondern daß Intersektionsbeziehungen ausreichen; dabei bleibt offen, wie weit diese Intersektionsbeziehungen zwischen den Basisformen der betreffenden Wörter gehen müssen: genügt etwa eine gemeinsame Buchstabenfolge von zwei Buchstaben? (Jede denkbare Regelung müßte hier das Verhältnis der gemeinsamen Buchstabenfolge zur Länge des Gesamtwortes berücksichtigen; allerdings kann dieses auch sehr kleine Werte annehmen, wie man aus dem Beispiel vazodiljatatory, vazokonstriktory, vazomotory ersieht -- von dem Problem der Abbreviaturen, die in unserem Korpus freilich nicht besonders stark vertreten sind, ganz zu schweigen.) Jedenfalls ist es ein Leichtes, in Wörterbüchern des Russischen Definitionen zu finden, deren Bestandteile zum jeweiligen Stichwort in einer formalen Intersektionsbeziehung stehen:

golen’: čast' nogi (u životnyx -- zadnej konečnosti) ot kolena do stopy. (ŠVE)

golova: čast' tela čeloveka (ili životnogo), sostojaščaja iz čerepnoj korobki $i$ lica (u životnogo mordy). (id.)

edok: odin čelovek kak edinica pri raspredelenii, rasxodovanii piščevyx produktov. (MAS).

Das reduktionistische Prinzip der getrennten Analyse von Ausdrucks- und Inhaltsseite stößt hier wieder an seine Grenzen; es wäre vergeblich, diese Mängel durch zusätzliche Regelungen beheben zu wollen.

by ložkovidnymi (otsjuda nazvanie) prikomevymi list jami".

${ }^{23}$ Diese Einsicht liegt auch der Benennungstheorie WUNDTS zugrunde, die von ROZWADOWSKI auf die Wortbildung übertragen wurde. Wir können diese interessanten Zusammenhänge an dieser Stelle nicht weiterverfolgen. $\mathrm{Cf}$. auch CASSIRER 1988: 251sq. SOwie KRONASSER 1968: 103. 


\subsubsection{Zusammenfassung und Aufspaltung von Lexemen}

Wir sind bislang dem Problem der Wortbildungshomonymie innerhalb der Wortfamilie ausgewichen, indem wir einfach die Entscheidungen des SSS (und damit die Entscheidungen der geläufigsten einsprachigen Wörterbücher des Russischen) übernahmen. Dieser Zustand ist mit einer semantischen Analyse, die von den Oppositionen innerhalb des Wortbildungsparadigmas ausgeht, nicht zu vereinbaren.

Die Definition der Wortfamilien ist so angelegt, daß echte Homonyme nie zu ein und derselben Wortfamilie zusammengefaßt werden können. Anders stehen die Dinge bei den sogenannten Wortbildungshomonymen: da es immer ein Lexem gibt, zu dem sie beide in inhaltlicher und formaler Beziehung stehen (wenn nicht sogar zwischen ihnen selbst eine inhaltliche Beziehung besteht), gehören sie ein und derselben Wortfamilie und unter Umständen auch ein und demselben Wortbildungsparadigma an. Auf diese Weise enthalten viele Paradigmen Lexeme, zwischen denen es keinen formalen, sondern nur einen inhaltlichen Unterschied gibt, wie das bereits erwähnte lopatka, und lopatka, in dem Wortbildungsparadigma von lopata; solche Lexeme wollen wir jetzt zu einem einzigen Lexem zusammenfassen. Dafür spricht auch die Tatsache, daß es, wie wir bereits gesehen haben, neben ihnen eine ganze Reihe Ableitungen gibt, deren Mehrdeutigkeit keine Aufspaltung in zwei Lexeme zur Folge hatte. Nach welchen Kriterien hier die Entscheidung der russischen Wörterbücher erfolgt, die sich im SSS niedergeschlagen hat, ist nicht eindeutig auszumachen.

In einer anderen Gruppe von Fällen liegt die umgekehrte Situation vor: Wörter, zwischen denen nicht nur ein semantischer, sondern auch ein formaler Unterschied besteht, werden im SSS zu einem einzigen Lexem zusammengefaßt. So führt etwa -um ein besonders krasses Beispiel zu nennen -- BASI das bereits erwähnte jabloćnik als polysemes Lexem mit zwei Bedeutungen an: 'Apfelverkäufer' und 'Kartoffelauflauf'. Einen formalen Niederschlag findet dieser Bedeutungsunterschied im Akkusativ, der für das beseelte Substantiv jabločnik, 'Apfelverkäufer' mit dem Genitiv zusammenfällt, für die Gerichtsbezeichnung jabločnik. 'Kartoffelauflauf' jedoch nicht. Die Opposition zwischen jabločnik, und jabločnik, erfüllt also das Kriterium der Relevanz (pertinence), da der semantische Unterschied zwischen diesen beiden Ableitungen (falls man auch jabloćnik. als Ableitung von jabloko ansehen will) zumindest in einigen Kontexten von einem formalen Unterschied begleitet wird. Diese Opposition geht verloren, wenn man hier der Entscheidung von BAS1 bzw. SSS folgt und die beiden Wörter zu einem Lexem zusammenfaßt.

Es bedarf kaum einer Erwähnung, daß der SSS auch in solchen Fällen die Inkonsequenzen seiner Quellen widerspiegelt: so wird etwa der Plural banki 'Schröpfköpfe' als eigenes Lexem angeführt, die Plurale ložki und tarelki (beides Bezeichnungen für Musikinstrumente) jedoch nicht; dabei ist der formale Unterschied zwischen ložka und lozki, tarelka und tarelki deutlicher als der zwischen banka und banki ausge- 
prägt, da letzteres Wort kaum als echtes plurale tantum angesehen werden darf. (Die Pluralform im SSS ist dadurch zu erklären, daß das Wort aus der BSE übernommen wurde.)

Für die Aufspaltung solcher Lexeme reicht der Begriff der formalen Identität, wie er in 2.2.1 im Anschluß an BERGER eingeführt worden ist, nicht aus: formale Identität besteht ja nicht nur zwischen lopatka, und lopatka, banka und banki, sondern auch zwischen jabloćnik, und jabločnik, ložka und ložki, tarelka und tarelki etc. Um hier eine Trennlinie zu ziehen, wird es notwendig sein, die formalen Beziehungen zwischen den Lexemen, die bislang allein auf der syntagmatischen Achse definiert wurden, auf die paradigmatische Achse der Sprache zu übertragen. Wir führen dazu die Begriffe der paradigmatischen formalen Inklusion und der paradigmatischen formalen Identität ein:

Zwei Lexeme A und B stehen zueinander in einer paradigmatischen (formalen) Inklusionsbeziehung, wenn jeder Wortform von A eine korrelative Wortform von B an die Seite gestellt werden kann, die mit ihr formal absolut identisch ist; man kann in diesem Fall sagen, A sei in B paradigmatisch enthalten. Falls sowohl A in B als auch $B$ in $A$ paradigmatisch enthalten ist, so besteht zwischen $A$ und $B$ eine paradigmatische (formale) Identitätsbeziehung.

Absolute formale Identität bedeutet, daß die beiden Wortformen nicht nur in allen Buchstaben ( $e$ und $\ddot{e}$ sollen dabei als verschiedene Buchstaben gelten), sondern auch in der Akzentstelle übereinstimmen. Zwischen den Wortformen múka und muká, nebo und nëbo besteht also keine absolute formale Identität, auch wenn die Basisformen der Lexeme, zu denen diese Wortformen gehören, im Sinne BERGERS formal identisch sind: die absolute formale Identität ist eine Beziehung zwischen Wortformen, die formale Identität eine Beziehung zwischen Lexemen, die durch den Vergleich ihrer Basisform bzw. der Varianten dieser Basisform ermittelt wird.

Mit Hilfe der paradigmatischen formalen Identität können nun die notwendigen Anpassungen an der Lemmatisierung des SSS vorgenommen werden:

a) wenn zwischen zwei oder mehreren Lexemen einer Wortfamilie paradigmatische formale Identität besteht, so werden sie zu einem Lexem zusammengefaßt;

b) wenn ein Lexem Bedeutungsvarianten aufweist, zwischen denen keine paradigmatische formale Identität besteht, so wird jede dieser Bedeutungsvarianten als separates Lexem behandelt.

Ad a): Es werden z.B. lopatka, 'Dem. zu lopata' und lopatka, 'Schulterblatt' und die entsprechenden Beziehungsadjektive lopatočnyj, und lopatočnyj, banka, 'Einweckglas' und banka, 'Schröpfkopf' zusammengefaßt, jedoch nicht ikristyj 'soderžaščij 
ikru' und ikristyj 's vypuklymi ikrami', da diese Lexeme verschiedenen Wortfamilien angehören. Die Bedingung, daß sich die absolute formale Identität auf alle Wortformen erstrecken muß, verhindert außerdem, daß z.B. gusjatnica 'rabotnica' und gusjatnica 'posuda', die sich ja im Akkusativ Plural voneinander unterscheiden, zu einem Lexem zusammengefaßt werden. Man beachte, daß das Kriterium der Identität der Basisformen hier zu locker sein würde. Entsprechendes gilt für Ableitungen, die sich nur in der Akzentstelle voneinander unterscheiden, etwa ambárišč 'uvel. $k$ ambar' vs. ambarišč 'mesto, byvšee pod ambarom' (BASI): da die absolute formale Identität auch die Identität der Akzentstelle voraussetzt, werden sie nicht miteinander verschmolzen. Dies ist äußerst wichtig, da ja der formalen Opposition in diesem Fall distinktive Funktion zukommt. Auch wenn von zwei bedeutungsverwandten, aber nicht bedeutungsgleichen Lexemen das eine Akzentschwankungen aufweist, die beim anderen fehlen, können sie nicht miteinander verschmolzen werden: ostrotá/ostróta 'ostroumnoe vyraženie' kann nicht mit ostrotá 'Abstraktum zu ostryj' zu einem Lexem zusammengefaßt werden, da das erste Wort Wortformen aufweist, die dem zweiten fehlen, z.B. den Genitiv Singular ostróty. Entsprechendes gilt schließlich für barašek 'jagnenok' und baraški 'belaja pena na grebnjax voln; nebol'šie kučevye oblaka' (MAS), da es in der Menge der Wortformen von baraški keine Entsprechung zu den Singularformen von barašek gibt.

Ad b): Nach dieser Regel werden jabločnik, und jabločnik, ložka und ložki, tarelka und tarelki in zwei Lexeme aufgespalten; außerdem Fälle wie katernyj, (Bez.-Adj. zu kater) und katernvj, (Matrose) granatovyj, (Bez.-Adj. zu granat) und granatovye. (Taxonym): zwar kann jeder Wortform von katernyj, bzw. granatovye: eine formal absolut identische korrelative Wortform von katernyj, bzw. granatovyj, an die Scite gestellt werden, das umgekehrte gilt jedoch nicht.

Regel b) ist nur als allgemeine Empfehlung, nicht als genaue Handlungsanweisung aufzufassen, da sich der Begriff der Bedeutungsvariante eines Lexems einer vollständigen Explikation widersetzt (cf. die Polemik gegen den verwandten Terminus der Bedeutungsnuance (ottenok značenija] bei APRESJAN 1974: 243sqq.). Schwierigkeiten bereiten in erster Linie die überaus vielfältigen semantischen Verschiebungen, die beim Übergang vom Singular zum Plural eintreten können (cf. die eingehende Analyse bei SOBOLEVA 1980: 128-216). In unserer Analyse nehmen wir die Aufspaltung eines Lexems nur in jenen Fällen vor, in denen bei einem der beiden Numeri eine deutliche Veränderung der lexikalischen Bedeutung stattgefunden hat, wenn also etwa tarelki als Bezeichnung für ein Musikinstrument, baraški als Bezeichnung für eine atmosphärische Erscheinung dient etc. Die Lexeme, deren Plural nur eine zusätzliche semantische Nuance aufweist, wie die Intensität bei boli, pereživanija oder die Ausdehnung (protjažennost) bei snega, tumany (in der Übersicht bei SOBOLEVA 1980: 135 ist dies der Zweig 01), werden dagegen nicht aufgespalten. 


\subsubsection{Definition des Wortbildungsparadigmas}

In diesem Kapitel stellen wir uns die Aufgabe, ein Verfahren anzugeben, das es erlaubt, den Bestand des Wortbildungsparadigmas eines unserer Ausgangssubstantive zu bestimmen, indem man seine Wortfamilie zum Ausgangspunkt nimmt. Dieses Verfahren ist auf unseren Gegenstand, die konkreten Substantive, abgestimmt; seine Anwendung auf Wortbildungsparadigmen von Verben oder Adjektiven würde zusätzliche Modifikationen verlangen, auf die wir in diesem Zusammenhang nicht einzugehen brauchen.

Eine erste Annäherung an den Begriff des Wortbildungsparadigmas wurde bereits in der Einleitung vorgenommen: wir verstehen darunter die Menge der Ableitungen eines Basiswortes, die auf der ersten Ableitungsstufe stehen, d.h. vom Basiswort nur um einen Ableitungsschritt entfernt sind. ${ }^{24}$ Unser Verfahren stellt einen Versuch dar, Wortbildungsparadigmen zu konstruieren, die dieser Definition genügen, ohne dafür den Begriff der Ableitungsbeziehung vollständig explizieren zu müssen, d.h. ohne vorher über alle Wortpaare einer Wortfamilie entscheiden zu müssen, ob zwischen ihren Mitgliedern eine Ableitungsbeziehung besteht oder nicht. (Man kann also sagen, daß der Begriff des Wortbildungsparadigmas in dieser Arbeit dem Begriff der Ableitungsbeziehung vorgeordnet ist). Zur Rechtfertigung eines solchen Verfahrens wollen wir uns zunächst die Schwierigkeiten vergegenwärtigen, die eine vollständige Explikation des Begriffs der Ableitungsbeziehung mit sich bringt. Wir betrachten dazu die Wortfamilien von jabloko und svekla.

Zwischen den Mitgliedern dieser Wortfamilien bestehen sehr unterschiedliche formale und inhaltliche Beziehungen. Was die formalen Beziehungen betrifft, so können sie wie in Unterkapitel 2.2.1 in (echte) Inklusion, Identität und Intersektion eingeteilt werden; in einigen Fällen von (syntagmatischer) formaler Identität erlaubt die Hinzuziehung des Flexionsparadigmas eine weitere Differenzierung -- je nachdem, ob

${ }^{24}$ Cf. die etwas weiter gefaßte Definition bei ZemSKAJA 1992: 17: "nabor proizvodnyx odnogo bazovogo slova, naxodjaščixsja na odnoj stupeni slovoobrazovanija". Das bedeutet etwa, daß vozžigatel'nica, samozažiganie, samozažigatel', zažigajušče, zažigatel'no, zažigatel'nost', zažigatel'nica, obžignyj, obžigovyj, obžigal'ščica, izvestkovoobžigatel'nyj, neobožžennost', otžigal'ščica, podžigatel'nica, podžigatel'stvo, podžigatel'skij, prožigatel'nica, prožigatel'skij, razžižka, musorosžiganie. samosožženec als Ableitungen vierter Stufe (nach TIX) von žec̆ das vierte (und letzte) Wortbildungsparadigma dieses Wortes bilden. Die Frage, ob Wortbildungsparadigmen höherer Ableitungsstufen noch den notwendigen semantischen und morphologischen Zusammenhalt aufweisen, um als Einheiten des Wortbildungssystems zu gelten, müßte Gegenstand einer eigenen Untersuchung werden. ZEMSKAJA selbst bezieht sich in der zitierten Arbeit ausschließlich auf die Wortbildungsparadigmen der ersten Ableitungsstufe. 
eine echte paradigmatische Inklusionsbeziehung vorliegt oder nicht. Man beachte jedoch, daß die paradigmatische Inklusionsbeziehung genau umgekehrt wie die syntagmatische behandelt werden muß: falls Lexem $A$ in Lexem $B$, nicht jedoch Lexem B in Lexem A paradigmatisch formal enthalten ist, so ist nicht $B$, sondern $A$ als das komplexere (weil kontextuell stärker gebundene) Lexem anzusehen.

Die Vielfalt der inhaltlichen Beziehungen wollen wir anhand der Definitionen illustrieren, die die vier wichtigsten einsprachigen russischen Wörterbücher (OŻE, MAS, BASI, UŚA) von den Mitgliedern der Wortfamilie von jabloko liefern. Es lassen sich hier folgende Fälle unterscheiden:

a) Es gibt eine Wörterbucherklärung des Lexems A, die das Lexem B enthält, und es gibt keine Wörterbucherklärung des Lexems B, die das Lexem A enthält. Man kann dies als Zeichen für eine echte Inklusionsbeziehung zwischen den Inhaltsseiten von $\mathrm{A}$ und $\mathrm{B}$ werten. Beispiele: $\mathrm{A}=$ jablokorezka, $\mathrm{B}=$ jabloko; $\mathrm{A}=$ jablonnik, $\mathrm{B}=$ jablonja.

b) Es gibt sowohl (mindestens) eine Wörterbucherklärung von $\mathrm{A}$, die $\mathrm{B}$ enthält, als auch (mindestens) eine Wörterbucherklärung von $\mathrm{B}$, die $\mathrm{A}$ enthält. Diesem Verhalten der Wörterbücher kann auf der Inhaltsseite der beiden Wörter sowohl eine Intersektions- als auch eine Identitätsbeziehung entsprechen. Wir geben ein Beispiel für eine inhaltliche Intersektionsbeziehung: jablonja und jabloko, die in USA folgendermaßen definiert werden: jabloko 'plod jabloni, obyčno šarovidnoj formy' vs. jablonja 'fruktovoe derevo iz sem. rozovyx, prinosjaščee jabloki' (zirkulär!), cf. MAS: jabloko 'plod jabloni' vs. jablonja 'plodovoe derevo sem. rozocvetnyx'.

c) Es gibt weder eine Wörterbucherklärung von A, die B enthält, noch eine Wörterbucherklärung von $B$, die $A$ enthält. In diesem Fall wird zwischen den Inhaltsseiten beider Wörter meist eine Intersektionsbeziehung bestehen. Beispiele: jablokorezka und jablonja, jablonnik und jabloko etc.

Die verschiedenen Beziehungstypen, die sich aus der Kombination der inhaltlichen mit den formalen Beziehungstypen ergeben, mag folgende Tabelle illustrieren: 


\begin{tabular}{||l|l|l|l|}
\hline & A $\subset$ B & B $\subset$ A & A = B \\
\hline $\begin{array}{l}\text { a1) (nur B durch A de- } \\
\text { finiert) }\end{array}$ & a & b & c \\
\hline $\begin{array}{l}\text { a2) (nur A durch B de- } \\
\text { finiert) }\end{array}$ & d & e & $\mathrm{f}$ \\
\hline $\begin{array}{l}\text { b) (B durch A und A } \\
\text { durch B definiert) }\end{array}$ & $\mathrm{g}$ & $\mathrm{h}$ & $\mathrm{i}$ \\
\hline $\begin{array}{l}\text { c) (weder B durch A } \\
\text { noch A durch B defi- } \\
\text { niert) }\end{array}$ & $\mathrm{j}$ & $\mathrm{k}$ & $\mathrm{l}$ \\
\hline
\end{tabular}

Dabei sollen $\mathrm{A} \subset \mathrm{B}$ und $\mathrm{B} \subset \mathrm{A}$ für die formale Inklusion (in der einen bzw. der anderen Richtung) stehen; die formale Identität und die formale Intersektion, die ja beides symmetrische Beziehungen sind und deshalb nicht die Bestimmung einer Ableitungsrichtung erlauben, werden zusammen betrachtet und mit $\mathrm{A}=\mathrm{B}$ gekennzeichnet. Auf der inhaltlichen Seite ist ebenfalls die antisymmetrische Beziehung a) in zwei Unterbeziehungen aufzuspalten (a1 und a2, je nachdem, ob in den Wörterbüchern $B$ durch $A$ oder $A$ durch $B$ definiert wird); zum Beziehungstyp b) rechnen wir auch jene Fälle, in denen die Wörterbücher eine Bedeutungsgleichheit zwischen zwei Ableitungen postulieren.

Es folgt eine Darstellung aller Beziehungen unter den Mitgliedern der Wortfamilie von jabloko in Form einer Matrix. Wir geben zunächst eine Liste der Mitglieder der Wortfamilie mit ihren Bedeutungen. In dieser Liste sind hinter jedem Wort in geschweiften Klammern jene Wörter der Wortfamilie aufgeführt, die in seinen Wörterbuchdefinitionen in OŽE, MAS, BASı und USA enthalten sind (bei jablonevye, das als eigenständiges Lemma in keinem dieser Wörterbücher belegt ist, wurde der Artikel in der BSE herangezogen). Jablonevyj und jablonnyj werden von den Wörterbüchern gleichgesetzt; deshalb ist sowohl jablonnyj hinter jablonevyj als auch jablonevyj hinter jablonnyj aufgeführt. Außerdem ist jabloko hinter jablonja und jablonja hinter jabloko aufgeführt, da, wie oben bereits festgestellt worden ist, sowohl eine Wörterbucherklärung von jabloko mit Hilfe von jablonja als auch eine von jablonja mit Hilfe von jabloko belegt ist (daß beide Erklärungen in demselben Wörterbuch [UŠA] vorkommen, ist in diesem Zusammenhang ohne Belang). Jabločnik 'Kartoffelauflauf' ist nach Regel b) von Unterkapitel 2.2.4 von jabločnik, 'Apfelverkäufer' getrennt worden und wird aus der Wortfamilie gestrichen, da seine inhaltliche Beziehung zu den übrigen Mitgliedern mehr als zweifelhaft ist und wir bei Artefak- 
ten, anders als bei natural kinds, keine ad-hoc-Definitionen zulassen wollen. (In unserem Material ist dies der einzige Fall, in dem eine Veränderung des Bestandes der Wortfamilie nach der Aufspaltung eines Lexems notwendig wird.)

1 jabloko: \{jablonja\} 'Apfel'

2 jablonevye: \{jablonja, jabloko\} 'Maloideae, Pomoideae, Pyroideae' 'Apfelartige' (BSE்) (Unterfamilie)

3 jablonevyj: (jablonja, jablonnyj\} 'Apfelbaum-'

4 jablonnik: \{jablonja\} 'Apfelgarten' ('mesto, gde rastut jabloni', BAS1)

5 jablonnyj: \{jablonja, jablonevyj\} 'Apfelbaum-'

6 jablonovka: \{jabloko\} 'schäumendes Getränk, das aus dem Saft von gekochten Äpfeln hergestellt wird' (BAS1) 'Apfelwein, Cider' (PAW)

7 jablon'ka: (jablonja) 'Dem. zu jablonja'

8 jablonja: \{jabloko\} 'Apfelbaum'

9 jabločko: |jabloko\} 'Dem. zu jabloko' 'Mittelpunkt der Zielscheibe' 'eine Art Matrosentanz'

10 jabločnik: \{jabloko\} 'Apfelverkäufer'

11 jabločnica: \{jabloko, jabločnik\} 'Apfelverkäuferin'

12 jabločnyj: |jabloko\} 'Apfel-'

13 jablokorezka: (jabloko\} 'Apfelschneidemaschine'

Es folgt die Matrix, in der die formalen und inhaltlichen Beziehungen zwischen den Mitgliedern der Wortfamilie dargestellt sind. Die Nummern in der ersten Spalte repräsentieren jeweils das Wort $A$, die Nummern in der ersten Zeile das Wort B; die Buchstaben drücken die Beziehung zwischen A und B aus. So steht z.B. im Kästchen, das der Spalte 3 und der Zeile 6 entspricht, der Buchstabe j, weil jablonevyj (3 $=$ Wort A) zu jablonovka $(6=$ Wort $\mathrm{B})$ in einer formalen Inklusionsbeziehung steht $(A \subset B)$ und keines der beiden Wörter in der Wörterbucherklärung des anderen enthalten ist. Die Matrix ist bezüglich der Diagonale symmetrisch, wobei folgende Entsprechungen gelten: $a / e, g / h, d / b, j / k, c / f, i / i, l / l$. 


\begin{tabular}{|c|c|c|c|c|c|c|c|c|c|c|c|c|c|}
\hline & 1 & 2 & 3 & 4 & 5 & 6 & 7 & 8 & 9 & 10 & 11 & 12 & 13 \\
\hline 1 & & c & 1 & 1 & 1 & c & 1 & $\mathrm{i}$ & $\mathbf{a}$ & $\mathbf{a}$ & $\mathbf{a}$ & $\mathbf{a}$ & $\mathbf{a}$ \\
\hline 2 & $\mathrm{f}$ & & 1 & 1 & 1 & $\mathbf{j}$ & 1 & e & 1 & 1 & 1 & 1 & 1 \\
\hline 3 & 1 & 1 & & 1 & $\mathbf{i}$ & $\mathbf{j}$ & 1 & $\mathbf{e}$ & 1 & 1 & 1 & 1 & 1 \\
\hline 4 & 1 & 1 & 1 & & $\mathrm{k}$ & 1 & 1 & e & 1 & 1 & 1 & 1 & 1 \\
\hline 5 & 1 & 1 & $\mathrm{i}$ & $\mathrm{j}$ & & 1 & 1 & $\mathrm{e}$ & 1 & 1 & 1 & 1 & 1 \\
\hline 6 & $\mathrm{f}$ & $k$ & k & 1 & 1 & & 1 & k & 1 & 1 & 1 & 1 & 1 \\
\hline 7 & 1 & 1 & 1 & 1 & 1 & 1 & & e & 1 & 1 & 1 & 1 & 1 \\
\hline 8 & $\mathrm{i}$ & $\mathbf{a}$ & $\mathbf{a}$ & $\mathbf{a}$ & $\mathbf{a}$ & $\mathbf{j}$ & $\mathbf{a}$ & & 1 & 1 & 1 & 1 & 1 \\
\hline 9 & $\mathrm{e}$ & 1 & 1 & 1 & 1 & 1 & 1 & 1 & & 1 & 1 & 1 & 1 \\
\hline 10 & e & 1 & 1 & 1 & 1 & 1 & 1 & 1 & 1 & & c & $\mathrm{k}$ & 1 \\
\hline 11 & e & 1 & 1 & 1 & 1 & 1 & 1 & 1 & 1 & $\mathrm{f}$ & & $\mathrm{k}$ & 1 \\
\hline 12 & e & 1 & 1 & 1 & 1 & 1 & 1 & 1 & 1 & $\mathbf{j}$ & $\mathbf{j}$ & & 1 \\
\hline 13 & e & 1 & 1 & 1 & 1 & 1 & 1 & 1 & 1 & 1 & 1 & 1 & \\
\hline
\end{tabular}

Falls man die paradigmatische Achse berücksichtigt, so kann man die formale Beziehung zwischen jablonevyj und jablonevye als gerichtet ansehen: jablonevye steht zu jablonevyj in der Beziehung der echten paradigmatischen Inklusion. In diesem Fall ist an der Schnittstelle von 2 und 3 nicht $l$, sondern $j / k$ zu setzen.

Als zweites Beispiel führen wir die Matrix der Wortfamilie von svekla an:

\section{I svekla: || 'Rübe'}

2 sveklovica: \{svekla\} 'Zuckerrübe'

3 sveklovičnica: \{sveklovica) 'Arbeiterin, die beim Anbau und bei der Ernte der Zuckerrübe beschäftigt ist'

4 sveklovičnyj: \{svekla, sveklovica, svekol'nyj\} 'Zuckerrüben'

5 sveklovišče: \{sveklovičnyj\} 'Zuckerrübenfeld' (sowohl BASı als auch MAS definieren es als 'sveklovičnoe pole', mit demselben Beispiel)

6 sveklovod: \{sveklovodstvo\}'Spezialist für Zuckerrübenbau' (so BIE, aber nach den Definitionen der russischen Wörterbücher: 'Spezialist für Rübenanbau')

7 sveklovodstvo: \{svekla\} '(Zucker)rübenanbau'

8 sveklovodčeskij: \{svekla, sveklovodstvo\} '(Zucker)rübenanbau-'

9 sveklokombajn: \{svekla\} 'Rübenemtemaschine, Rübenkombine' 
10 sveklosaxarnyj: \{svekla, sveklovica\} 'Zuckerrüben-'

11 sveklosovxoz: \{svekla, sveklovica\} 'Sowjetgut, in dem Rüben angebaut werden'

12 svekolka: \{\}$^{25}$ 'Dem. zu svekla'

13 svekol'nik: \{svekla, svekol'nyj\} 'Rübensuppe' 'Rübenkraut, Blätter der Rübe'

14 svekol'nyj: \{svekla, sveklovičnyj\} 'Rüben'

15 podsvekol'nik: | $\mid$ '(Amarantus retroflexus) bogiger Fuchsschwanz'

Die Wortfamilie enthält eine Reihe von Komposita, die sich ganz wie sveklokombajn verhalten:

a) ihre Erklärungsmenge enthält nur das Wort svekla und

b) sie stehen in einem formalen Intersektionsverhältnis zu allen übrigen Mitgliedem der Kategorie außer svekla, zu dem sie in einem Inklusionsverhältnis stehen.

Es handelt sich um folgende Wörter: sveklokopatel, sveklomojka, sveklopogruzčik, sveklopod-emnik, sveklopriemočnyj, sveklorezka, sveklosemena, sveklosejuščj, sveklosejanie, sveklouborka, sveklouboročnyj, svekloutomlenie. Um die Tabellen nicht allzu unübersichtlich werden zu lassen, haben wir diese Wörter nicht in die Aufstellung aufgenommen. Ihre Zeile bzw. Spalte in der Matrix wäre mit der von sveklokombajn identisch.

${ }^{25}$ Dieses Wort ist nach Ausweis des SSS nur im Orfoèpičeskij slovar belegt, der keine Definitionen enthält. Wir wollen hier strikt den Wörterbüchern folgen und werden deshalb nicht die naheliegende Korrektur zu \{svekla\} vornehmen. 


\begin{tabular}{|c|c|c|c|c|c|c|c|c|c|c|c|c|c|c|c|}
\hline & 1 & 2 & 3 & 4 & $s$ & 6 & 7 & 8 & 9 & 10 & 11 & 12 & 13 & 14 & is \\
\hline 1 & & $\mathbf{a}$ & $\mathbf{j}$ & $\mathbf{a}$ & $\mathrm{j}$ & $\mathrm{j}$ & $\mathbf{a}$ & $\mathbf{a}$ & $\mathbf{a}$ & $\mathbf{a}$ & $\mathbf{a}$ & $\mathrm{j}$ & $\mathbf{a}$ & $\mathbf{a}$ & $\mathrm{j}$ \\
\hline 2 & e & & $\mathbf{a}$ & $\mathbf{a}$ & 1 & 1 & 1 & 1 & 1 & c & c & 1 & 1 & 1 & 1 \\
\hline 3 & $\mathrm{k}$ & $\mathbf{e}$ & & $\mathbf{k}$ & 1 & 1 & 1 & 1 & 1 & 1 & 1 & 1 & 1 & 1 & 1 \\
\hline 4 & e & e & $\mathrm{j}$ & & c & 1 & 1 & 1 & 1 & 1 & 1 & 1 & 1 & $\mathrm{i}$ & 1 \\
\hline 5 & $k$ & 1 & 1 & $\mathbf{f}$ & & 1 & 1 & 1 & 1 & 1 & 1 & 1 & 1 & 1 & 1 \\
\hline 6 & $\mathbf{k}$ & 1 & 1 & 1 & 1 & & $\mathrm{~d}$ & $\mathrm{j}$ & 1 & 1 & 1 & 1 & 1 & 1 & 1 \\
\hline 7 & $\mathrm{e}$ & 1 & 1 & 1 & 1 & b & & $\mathrm{c}$ & 1 & 1 & 1 & 1 & 1 & 1 & 1 \\
\hline 8 & e & 1 & 1 & 1 & 1 & k & $\mathbf{f}$ & & 1 & 1 & 1 & 1 & 1 & 1 & 1 \\
\hline 9 & e & 1 & 1 & 1 & 1 & 1 & 1 & 1 & & 1 & 1 & 1 & 1 & 1 & 1 \\
\hline 10 & $\mathbf{e}$ & f & 1 & 1 & 1 & 1 & 1 & 1 & 1 & & 1 & 1 & 1 & 1 & 1 \\
\hline 11 & $\mathbf{e}$ & $\mathrm{f}$ & 1 & 1 & 1 & 1 & 1 & 1 & 1 & 1 & & 1 & 1 & 1 & 1 \\
\hline 12 & $\mathrm{k}$ & 1 & 1 & 1 & 1 & 1 & 1 & 1 & 1 & 1 & 1 & & 1 & 1 & 1 \\
\hline 13 & $\mathrm{e}$ & 1 & 1 & 1 & 1 & 1 & 1 & 1 & 1 & 1 & 1 & 1 & & $\mathbf{e}$ & $\mathrm{j}$ \\
\hline 14 & e & 1 & 1 & $\mathrm{i}$ & I & 1 & 1 & 1 & 1 & 1 & 1 & 1 & $\mathbf{a}$ & & $\mathrm{j}$ \\
\hline 15 & $k$ & 1 & 1 & 1 & 1 & 1 & 1 & 1 & 1 & 1 & 1 & 1 & $\mathrm{k}$ & $\mathrm{k}$ & \\
\hline
\end{tabular}

Jeder der Buchstaben a) bis l) steht für eine bestimmte Relation über der Menge W der Mitglieder der Wortfamilie; dabei sind jeweils die Relationen a und $\mathrm{e}, \mathrm{g}$ und $\mathrm{h}$, $d$ und $b, j$ und $k, c$ und $f$ zueinander konvers; $i$ und $l$ sind symmetrisch, d.h. die Konversen ihrer selbst. Bei der Definition einer Ableitungsbeziehung geht es letzten Endes darum, aus diesen Relationen eine weitere Relation, eben die Ableitungsrelation, zu konstruieren. ( $\mathrm{Da}$ zweistellige Relationen mathematisch betrachtet nichts anderes als Untermengen des kartesischen Produktes $W \times W$, d.h. Mengen geordneter Paare sind, stellt sich diese Konstruktion als Durchführung der elementaren mengentheoretischen Operationen dar: A $\cap$ B, A $\cup$ B, A $\backslash$ B, Komplement von B). Wir betonen den konstruktiven Aspekt dieses Vorgehens: mit der Definition der Ableitungsbeziehung überschreitet man zwar keine absolute Grenze zwischen "sprachlichen Tatsachen" und "linguistischen Konstrukten" (eine solche Auffassung verbietet sich schon allein aus dem Grunde, daß die Wörterbucherklärungen, auf denen die oben dargestellten inhaltlichen Beziehungen beruhen, selber Ergebnisse einer wenn auch oft nur impliziten sprachwissenschaftlichen Interpretation sind), wohl aber die Grenze zwischen dem lexikographischen Material und seiner Inter- 
pretation im Lichte der Wortbildungslehre. Es ist wichtig, sich klarzumachen, daß jede Definition von Ableitungsbeziehungen dadurch, daß sie einige der Beziehungstypen a) bis I) in den Vordergrund stellt, einige nur ersatzweise heranzieht und andere schließlich ganz ausklammert, eine Interpretation des lexikologischen Ausgangsmaterials darstellt, die nur in bezug auf ein bestimmtes Untersuchungsziel als richtig oder falsch angesehen werden darf.

Welche Anforderungen werden nun im allgemeinen an die Ableitungsrelation $\mathrm{T} \subseteq \mathrm{W} \times \mathrm{W}$ gestellt?

a) Sie sollte eine Halbordnung sein, d.h. reflexiv (s.o.), transitiv (s.o.) und antisymmetrisch (aus $x T y$ und $y T x$ sollte $x=y$ für alle $x, y \in W$ folgen) (ERNÉ 1982: 46).

b) Die Menge W sollte T-zusammenhängend sein. Das bedeutet, daß nicht einzelne Wörter oder ganze Wortgruppen aus der Wortfamilie herausfallen dürfen (ERNE 1982: 57).

c) Die Menge $W$ sollte durch die Ableitungsrelation $T$ nach unten gerichtet sein. Eine Menge $W$ heißt durch $T$ nach unten gerichtet, falls es zu allen $x, y \in W$ ein z mit zTx und zTy gibt (ERNE 1982: 47). In einer Wortfamilie ist dies erfüllt, wenn es ein Wort gibt, von dem alle anderen Wörter der Wortfamilie (direkt oder indirekt) abgeleitet sind.

Wir wollen hier nur auf a) eingehen, d.h. auf die Forderung, daß die Ableitungsbeziehung eine Halbordnung sein soll, und die damit verbundenen Schwierigkeiten aufzeigen.

Die Reflexivität ist trivial, da sie durch einfache Setzung hergestellt werden kann (wir brauchen dazu nur zu bestimmen, daß jedes Wort als von sich selber abgeleitet gelten kann, d.h. die in den obigen Matrizen leergebliebenen Kästchen in der Diagonale in die Relation aufzunehmen). Auch die Forderung der Transitivität kann auf diese Weise erfüllt werden, solange man sich dabei im klaren bleibt, daß sie eher eine aus Gründen der linguistischen Modellierung an das Wortbildungssystem herangetragene Forderung als eine sprachliche Gegebenheit darstellt (cf. die Gegenbeispiele von JACENKO 1958: 19sq.).

Der Forderung der Antisymmetrie, d.h. der Gerichtetheit der Ableitungsbeziehungen, kann am schwierigsten Genüge geleistet werden. Betrachtet man unter diesem Gesichtspunkt die Relationen a) bis I), so kann man folgende Fälle unterscheiden:

a) Die Beziehung ist sowohl auf der Inhalts- als auch auf der Ausdrucksseite gerichtet, wobei beide Richtungen übereinstimmen. Dies ist der klassische Fall der Ableitung, bei der das ableitende Wort zum abgeleiteten sowohl formal als auch 
inhaltlich in einer echten, d.h. antisymmetrischen Inklusionsbeziehung steht [Relationen a) und e)], Beispiele: jabloko $\rightarrow$ jablokorezka, svekla $\rightarrow$ svekol'nik etc. Die Festlegung der Ableitungsrichtung bereitet in diesem Fall keine Schwierigkeiten.

b) Die Beziehung ist entweder nur auf der Inhaltsseite oder nur auf der Ausdrucksseite gerichtet:

b1) Auf der Inhaltsseite: Relationen c/f, wie in sveklovičnyj $\rightarrow$ sveklovišče, sveklovica $\rightarrow$ sveklosaxarnyj, jabloko $\rightarrow$ jablonovka.

b2) Auf der Ausdrucksseite: Relationen $\mathrm{g} / \mathrm{h}, \mathrm{j} / \mathrm{k}$, wie in lisa $\rightarrow$ lisica, jablonja $\rightarrow$ jablonovka etc.

Auch in diesem Fall läßt sich eine Ableitungsrichtung auf eindeutige, wenn auch z.T. nicht besonders einleuchtende Weise bestimmen.

c) Die Beziehung ist weder auf der Inhaltsseite noch auf der Ausdrucksseite gerichtet: Relationen $\mathrm{i}$ und 1, wie etwa in jabloko und jablonja, jablonnyj und jablonevyj, jablon'ka und jabločnik, sveklovišče und svekol'nik etc. Wie das erste Beispiel zeigt, kommt man in manchen dieser Wortpaare doch nicht umhin, eine Ableitungsrichtung zu definieren.

d) Die Beziehung ist auf beiden Seiten gerichtet, doch stimmen die Richtungen nicht überein, wie etwa in sveklovod und sveklovodstvo.

Offensichtlich erfordert die Festlegung der Ableitungsrichtung in den beiden letzten Kategorien die Hinzuziehung zusätzlicher Kriterien. Es ist hier nicht der Ort, die vielen unterschiedlichen Lösungen, die für diese Frage in der Wortbildungslehre schon vorgeschlagen wurden, zu referieren; wir verweisen auf den Überblick bei LOPATIN 1979. Auch BERGER hat in seiner bereits mehrfach zitierten Untersuchung versucht, durch die Aufstellung eines komplizierten Regelapparates in möglichst vielen Fällen eine Ableitungsrichtung zwischen den Wörtern der Wortfamilie festzulegen. Dieser Versuch ist leider, wie man bei der Anwendung der Regeln sehr bald feststellt, letztlich doch als gescheitert anzusehen; wir geben dafür drei Belege:

a) BERGER stellt auf p. 177 folgende Regel auf, die wegen ihrer Wichtigkeit hier in vollem Wortlaut zitiert werden soll:

Gegeben seien zwei Wörter A und B, die derselben 'Familie' angehören. Wenn A formal in B enthalten ist, sagen wir, daß B im weiteren Sinn von A abgeleitet ist. 
Das Bemerkenswerte -- und Gefährliche -- an dieser Regel ist, daß sie auf die Inhaltsseite der beiden Wörter keinerlei Bezug nimmt: eine formale Inklusionsbeziehung zwischen zwei Wörtern einer Wortfamilie genügt bereits, um zwischen ihnen auch eine Ableitungsbeziehung im weiteren Sinne zu postulieren. Zusammen mit BERGERs (von uns oben außer Kraft gesetzter) Regel, nach der zur Gewinnung der Variante einer Basisform auslautendes $n$ getilgt werden darf, führt das dazu, daß zwischen domina und domik eine Ableitungsbeziehung im weiteren Sinn besteht: domik ist von domina i. w. S. abgeleitet, da man aus der Basisform domin- die Variante domi- gewinnen kann, die in domik- enthalten ist, und das inhaltliche Verhältnis beider Wörter keine Rolle spielt. Da umgekehrt die Basisform von domik in der von domina nicht enthalten ist, fällt die Ableitungsbeziehung im weiteren Sinn mit der im engeren Sinn in diesem Fall zusammen (p. 180), d.h. domik ist von domina abzuleiten und damit von dom um zwei Ableitungsschritte entfernt.

Auch wenn man die Tilgung von $n$ nicht zuläßt, bleiben genug Ableitungspaare übrig, um Kopfzerbrechen zu bereiten: ponožovščik ist von nožovščik abgeleitet; gusenok und gusenyš von gusynja oder gusinyj; gusjačij von gusak oder gusek; etc.

b) Den Ausführungen p. 181sq. kann man entnehmen, daß zwischen pisat $\rightarrow$ perepisat ${ }^{\prime}$ einerseits und $x^{\prime}$ odit $^{\prime} \rightarrow$ perexodit $^{\prime}$ andererseits dieselben formalen und inhaltlichen Beziehungen bestehen. Was das konkret bedeuten soll, bleibt unklar; man kann jedoch p. 166 heranziehen, auf der die Äquivalenz zwischen Motivationsbeziehungen (d.h. inhaltlichen Beziehungen im Sinne BERGERs) folgendermaBen definiert ist:

Die Motivationsbeziehung zwischen den Wörtern A und B ist äquivalent der zwischen $C$ und $D$, wenn es eine Umschreibung der Bedeutung von A gibt, in der $B$ vorkommt und aus der man eine Umschreibung der Bedeutung von $C$ erhält, wenn man B in dem betreffenden Satz durch D ersetzt.

Es ist offensichtlich, daß eine solche Umschreibung für perepisat und perexodit (allein schon wegen des Aspektunterschiedes und der Notwendigkeit, perexodit unter Rückgriff auf ein determiniertes Fortbewegungsverb zu definieren) nicht gegeben werden kann. (Damit soll nicht geleugnet werden, daß es sich bei pere- in beiden Verben um "dasselbe Präfix" handelt, das in zwei verschiedenen Bedeutungsvarianten auftritt; die Frage, wie solche Bedeutungsvarianten ausgegliedert werden sollen und durch welches Modell ihrer Verwandtschaft am besten Rechnung getragen werden kann, ist jedoch äußerst kompliziert und darf deshalb keinesfalls in einem frühen Stadium der Wortbildungsanalyse als gelöst vorausgesetzt werden.) Ähnliche Probleme treten in sehr vielen Wortpaaren auf -- darunter auch in BERGERs nächstem Beispiel, sistema $\rightarrow$ sistematika und tema $\rightarrow$ tematika (man beachte die Definitionen der beiden Ableitungen im MAS). 
c) Auch auf der formalen Seite liegen die Dinge nicht besser. Nach BERGER besteht $z$ wischen perepis/perepisat einerseits und perexodit/perexod andererseits keine identische formale Beziehung. Die Entscheidung ist intuitiv einsichtig, da sich die beiden Ableitungen im Flexionsparadigma und durch die Palatalität/Nichtpalatalität des auslautenden Konsonanten unterscheiden, aber im Rahmen der aufgestellten Regeln kaum zu begründen: die formalen Unterschiede zwischen perexod und perepis' verschwinden, wenn man lediglich deren Basisformen perexodund perepis- betrachtet.

Weitaus großzügiger wird das Kriterium der formalen Parallelität dagegen im Fall von redkij/redet vs. červivyj/červivet' oder rybak/rybačit' vs. vyxod/vyxodit' (p. 186) ausgelegt. Hier wird eine Identität der formalen Beziehung postuliert, obwohl die Basisformen des ableitenden Wortes im jeweils ersten Wortpaar nach den Regeln der $\mathrm{p}$. 171 sq. verändert werden müssen, bevor man das Affix anfügen kann (bei redkij z.B. muß man auf den Komparativ zurückgreifen, um das $k$ loszuwerden; anschließend ist das $z$ wieder in $d$ umzuwandeln).

Die entscheidende Frage ist also hier, ob die Operationen, die man mit einer Basisform durchführt, bevor man den Formanten anfügen (bzw. ihre Inklusionsbeziehung zu einer anderen Basisform feststellen) kann, bei der Ermittlung der formalen Parallelität mitzählen oder nicht. In der Definition der Wortbildungsmuster, die an einer späteren Stelle der Arbeit (p. 195) gegeben wird, sind diese Operationen jedenfalls berücksichtigt worden, in den soeben zitierten Beispielen nicht.

Die erwähnten Schwierigkeiten, denen man leicht weitere an die Seite stellen kann, haben eine tiefere methodische Ursache: zur Festlegung der Ableitungsrichtung in einem Wortpaar, das unter die Kategorien c) und d) fällt, erweist es sich als notwendig, weitere Wortpaare heranzuziehen, die in formaler und/oder inhaltlicher Hinsicht mit ihm vergleichbar sind. Damit ist das reduktionistische Prinzip der getrennten Analyse der Wortpaare verletzt und ein Vorgriff auf die nächsthöhere Untersuchungsebene, die Ermittlung von Wortbildungstypen durch ein syntagmatisches oder ein paradigmatisches Verfahren, vollzogen: jeder Begriff von "Ähnlichkeit" oder "Parallelität" der formalen und/oder inhaltlichen Beziehung zwischen Wortpaaren setzt ja gewisse Vorstellungen von Wortbildungskategorien und Wortbildungsformanten voraus.

Aus diesen Gründen haben wir uns entschlossen, bei der Bestimmung des Wortbildungsparadigmas lediglich auf solche Wortpaare zurückzugreifen, bei denen die Ableitungsrichtung eindeutig feststeht. Wir gehen dabei von folgenden Beobachtungen aus:

a) Die meisten Wortfamilien der konkreten Substantive haben eine flache Struktur, bei der die höheren Ableitungsstufen relativ schwach besetzt sind; und 
b) Wortbildungspaare, die sich der Festlegung einer Ableitungsrichtung widersetzen, sind auf den höheren Ableitungsstufen denominaler Wortfamilien weniger stark vertreten als auf der ersten; bei Ableitungen abgeleiteter Wörter stimmen formale und inhaltliche Komplexität in der Regel überein. ${ }^{26}$

Diese Beobachtungen legen nahe, bei der Bestimmung des Wortbildungsparadigmas negativ vorzugehen: es sollen nicht jene Wörter bestimmt werden, die vom Grundwort unmittelbar abgeleitet worden sind und deshalb in sein Wortbildungsparadigma aufgenommen werden müssen, sondern umgekehrt jene Wörter, die von einem anderen Wort der Wortfamilie abgeleitet sind und deshalb aus dem Wortbildungsparadigma des Grundwortes auszuschließen sind. Den Sinn dieses Verfahrens kann man sich an folgendem Beispiel verdeutlichen:

\section{ljaguška $\rightarrow$ ljagušonok $\rightarrow$ ljagušonoček}

Es ist viel einfacher, ljagušonoček aus dem Wortbildungsparadigma von ljaguška auszuschließen, als ljagušonok darin aufzunehmen: ljagušonok ist in ljagušonoček sowohl formal als auch semantisch enthalten; die Aufstellung der Ableitungsbeziehung ljaguška $\rightarrow$ ljagušonok setzt dagegen eine spezielle Regel zur Tilgung des auslautenden $-k$ - in ljaguška voraus.

Bei den Wörtern, die auf diese Weise aus dem Wortbildungsparadigma des Grundwortes ausgeschlossen werden, machen wir eine bedeutsame Ausnahme: Adjektive dürfen als Zwischenglieder die Aufnahme der von ihnen direkt abgeleiteten Wörter in das Paradigma des Grundwortes nicht verhindern. Die Rechtfertigung für die Aufnahme von Ableitungen einer höheren Ableitungsstufe in das Wortbildungsparadigma eines Substantivs, falls das Zwischenglied ein (Beziehungs)adjektiv ist, besteht in der semantischen Neutralität der meisten dieser Adjektive, die ja nur das Vorhandensein einer erst durch den Sprachgebrauch mit bestimmtem Inhalt aufgefüllten Beziehung zwischen ihrer Ableitungsbasis und dem von ihnen determinierten Substantiv signalisieren. (Wir gehen im nächsten Kapitel auf die Problematik der Beziehungsadjektive näher ein; cf. AG-80, Bd. 1, p. 166, § 284, ScHUPBACH 1975: 67-70 sowie die Ausführungen bei ZEMSKAJA 1992: 36-39.) Da die Vorstellung einer solchen abstrakten Beziehung als kleinster gemeinsamer Nenner jeder Ableitung zugrundeliegt, scheint das adjektivische Mittelglied zur Semantik des abgeleiteten Wortes, das ihm ja formal durchaus näher steht, nichts beizutragen. (Es ist also nicht ganz genau, wenn man sagt, solche Ableitungen stünden dem ableitenden Substantiv semantisch näher als seinem Beziehungsadjektiv: dies können sie schon deshalb nicht, weil sich die reinen Beziehungsadjektive vom Substantiv semantisch gar nicht entfemt haben.) In den Wörterbüchern werden solche Ableitun-

${ }^{26}$ Im Rahmen generativer Wortbildungsmodelle wird dieser Tatsache durch den Mechanismus des level ordering Rechnung getragen. 
gen teils unter Rückgriff auf das Basissubstantiv, teils mit Hilfe des Adjektivs umschrieben. Unsere Regelung befreit uns von der Notwendigkeit, diese Inkonsequenz zu beseitigen, da sie für den Bestand der Wortbildungsparadigmen nunmehr keine Rolle spielt. Dagegen fallen die wenigen deadjektivischen Ableitungen, die ihrer unmittelbaren Ableitungsbasis semantisch tatsächlich näher stehen als dem zugrundeliegenden Substantiv und die wir nun auch in dessen Wortbildungsparadigma aufnehmen müssen, kaum ins Gewicht.

Ein gewisses Problem bilden jedoch Univerbate, da bei ihnen nicht das einfache Adjektiv, sondern eine Mehrwortbenennung als Zwischenglied auftritt (abrikosovaja nastojka $\rightarrow$ abrikosovka); sie könnten nur ausgeschlossen werden, wenn a) unser Korpus solche Mehrwortbenennungen enthielte und b) exakte Kriterien vorlägen, nach denen entschieden werden könnte, wann eine solche Mehrwortbenennung als Zwischenglied unentbehrlich ist und wann nicht (die Ausdrucksseite des Substantivs nastojka ist ja in der Ableitung nicht mehr vorhanden). Auch in diesem Bereich tritt das Phänomen auf, daß durch analogische Erweiterung eine bestimmte Ableitungsstufe in anderen Wortfamilien übersprungen wird: vergleiche mit dem vorhergehenden Beispiel jablonovka, das kein ?jablonovaja nastojka neben sich hat.

Und nun die Regeln, nach denen die Wortbildungsparadigmen der konkreten Substantive zu bestimmen sind:

! Man bestimme als erstes, welches Wort der Wortfamilie als primär (nichtabgeleitet) anzusehen ist.

Anmerkung 1: Dies ist die erste Entscheidung, die getroffen werden muß, und sie kann selbst nicht mehr begründet werden. Nichts spricht übrigens dagegen, abwechselnd verschiedene Wörter der Wortfamilie als primär anzusehen und ihre Wortbildungsparadigmen nach unserem Algorithmus zu konstruieren; man denke nur an jabloko und jablonja. (In diesen Fällen wird jedoch eine Sonderregel notwendig, die wir unten bei der Diskussion von vorona und voron einführen werden.)

Anmerkung 2: Wir behalten uns vor, in Einzelfällen mehrere Lexeme der Wortfamilie zu einem Lexem zusammenzufassen (als Varianten eines Lexems anzusehen), um dieses Lexem dann als primär anzusehen und sein Wortbildungsparadigma zu konstruieren. Diese Lösung wird in Fällen wie avtomobil/avto, taksomotor/taksi, skovorodka/skovoroda gewählt, um zu verhindern, daß das kürzere Wort als Zwischenglied andere Wörter aus dem Wortbildungsparadigma ausschließt.

? Man stelle fest, ob dieses Wort eines der konkreten Substantive ist, deren Wortbildungsparadigma konstruiert werden soll, oder nicht. 
Fall 1: Das primäre Wort der Wortfamilie ist das konkrete Substantiv, dessen Wortbildungsparadigma konstruiert werden soll. In diesem Fall geht man alle Wörter der Wortfamilie durch und behandelt sie nach folgendem Algorithmus (im folgenden sollen das primäre Wort durch $\mathrm{A}$, das untersuchte Wort durch $\mathrm{C}$ und eventuelle Zwischenglieder durch B symbolisiert werden):

? Gibt es ein anderes Wort der Wortfamilie (B), so daß folgende Bedingungen gelten:

a) $\mathrm{B}$ ist kein Adjektiv

und

b1) B steht zu C in der Beziehung der echten syntagmatischen formalen Inklusion ( $B$ ist in $C$ formal-syntagmatisch enthalten, $C$ ist nicht in $B$ formalsyntagmatisch enthalten)

oder

b2) C steht zu B in der Beziehung der echten paradigmatischen formalen Inklusion ( $\mathrm{C}$ ist in $\mathrm{B}$ formal-paradigmatisch enthalten, $\mathrm{B}$ ist nicht in $\mathrm{C}$ formalparadigmatisch enthalten)?

Fall 1.1: Ein solches Wort gibt es nicht. Dann ist $C$ in das Wortbildungsparadigma von $A$ aufzunehmen.

Fall 1.2: Es gibt eines (oder mehrere) solcher Wörter. Dann ist mit jedem von ihnen auf folgende Weise zu verfahren:

? Kann der Bedeutungsinhalt von $\mathrm{C}$ durch einen russischen Satz umschrieben werden, der $B$ enthält?

Fall 1.2.1: Ja, es gibt eine solche Paraphrase. Es ist dann weiter zu fragen:

? Kann auch der Bedeutungsinhalt von B durch einen russischen Satz umschrieben werden, der $C$ enthält?

Fall 1.2.1.1: Ja, es gibt eine solche Paraphrase. In diesem Fall ist das Wort C in das Wortbildungsparadigma von A aufzunehmen.

Fall 1.2.1.2: Eine solche Paraphrase ist nicht möglich. In diesem Fall ist das Wort $\mathrm{C}$ aus dem Wortbildungsparadigma von $\mathrm{A}$ auszuschließen.

Anmerkung 3: Was die Paraphrasen (Bedeutungsumschreibungen) angeht, so ist hier, wo es um die endgültige Bestimmung des Bestandes des Wortbildungsparadigmas geht, ein weitaus strengerer Maßstab anzulegen als bei der Zusammenfassung der Wörter zu Wortfamilien. Die Umschreibung soll nicht einfach denkbar, sondern naheliegend und in flüssigem Russisch formulierbar 
sein. Es versteht sich von selbst, daß man bei der Umschreibung von B durch $C$ nicht strengere Kriterien anwenden kann als bei der Umschreibung von $C$ durch B: falls man die Umschreibung von sveklovodstvo durch sveklovod für zulässig erklärt, so muß es a fortiori auch die von sveklovod durch sveklovodstvo sein.

Anmerkung 4: Die Paraphrasierung von $\mathrm{C}$ durch $\mathrm{B}$ wird auch dann als unmöglich angesehen, wenn $C$ mehrere Bedeutungen hat, von denen nur ein Teil durch B paraphrasiert werden kann. Sc führt etwa USA unter ložečnik zwei Bedeutungen an: 'to že, čto ložkar' [= rabočij, kustar', delajuščij derevjannye ložki]' und 'igrajuščij na ložkax (vo 2 znač. [= primitivnyj muzykal'nyj instrument vrode kastan'et])'. In der zweiten Bedeutung ist ložečnik durch ložki paraphrasierbar, in der ersten jedoch nicht; es wird deshalb nicht aus dem Wortbildungsparadigma von ložka ausgeschlossen.

Fall 1.2.2: Die Paraphrase von $C$ durch $B$ ist nicht möglich. Auch in diesem Fall ist $\mathrm{C}$ in das Wortbildungsparadigma von $\mathrm{A}$ aufzunehmen.

Fall 2: Das primäre Wort der Wortfamilie ist mit dem konkreten Substantiv, das untersucht werden soll, nicht identisch; d.h. das konkrete Substantiv ist als abgeleitet anzusehen. In diesen nicht besonders häufigen Fällen ist unser Prinzip umzukehren: es werden nur jene Wörter in das Wortbildungsparadigma des betreffenden Substantivs aufgenommen, in denen es formal und inhaltlich enthalten ist; unechte Inklusion (d.h. formale und/oder inhaltliche Identität) soll jedoch in diesem Fall kein Hindernis für die Aufnahme des Wortes in das Wortbildungsparadigma sein. Wir gehen also alle Wörter der Wortfamilie durch und fragen:

? Ist das konkrete Substantiv A in B formal enthalten?

Fall 2.1: Eine formale Inklusion liegt nicht vor. Dann besteht kein Grund, $B$ in das Wortbildungsparadigma von A aufzunehmen.

Fall 2.2: Eine formale Inklusion liegt vor. Dann fragen wir weiter:

? Kann B durch einen russischen Satz umschrieben werden, der A enthält?

Fall 2.2.1: Die Antwort ist positiv. Dann ist B in das Wortbildungsparadigma von A aufzunehmen.

Fall 2.2.2: Die Antwort ist negativ. Dann ist $B$ in das Wortbildungsparadigma von $A$ nicht aufzunehmen. 
Anmerkung 5: Es ist möglich, daß das nach Fall 2 konstruierte Wortbildungsparadigma eines abgeleiteten konkreten Substantivs auch Ableitungen nicht der ersten, sondern höherer Ableitungsstufen (von diesem Substantiv aus gezählt) enthält. In diesem Fall ist es als eine Art Unterfamilie anzusehen, auf die der Algorithmus von Fall 1 anzuwenden ist; die Entscheidung darüber muß allerdings intuitiv getroffen werden. S. weiter unten zu posuda.

Anmerkung 6: Die Anforderungen, die an die Umschreibungen gestellt werden, sind unter Fall 2 dieselben wie unter Fall 1.

\section{Beispiele:}

1. Das Wortbildungsparadigma von jabloko lautet:

jablonovka
jabločko
jabloćnik
jabločnica
jabločnyj
jablokorezka
jablonja

Ausgeschlossen werden jablonnik 'Apfel(baum)garten', weil jablonja in ihm formal und inhaltlich (echt) enthalten ist; desgleichen jablonevyj, jablonnyj, jablonevye. Jablonovka wird dagegen nicht ausgeschlossen: zwar ist jablonja in jablonovka formal enthalten, doch kann jablonovka durch jablonja nicht auf natürliche Weise definiert werden. Jabločnica wird aufgenommen, weil zwischen jabločnik und jabloćnica keine formale Inklusion, sondern Identität besteht.

Wenn man jablonja als nichtabgeleitet ansieht, erhält man folgendes Paradigma:

jablonevyj
jablonnyj
jablonevye
jablonnik
jablonka
jabloko

Jablonnyj ist in jablonnik formal-syntagmatisch enthalten, und wahrscheinlich kann man jablonnik durch jablonnyj umschreiben (das Gegenteil ist natürlich nicht möglich); trotzdem wird jablonnik aus dem Wortbildungsparadigma nicht ausgeschlossen, 
da jablonnyj ein (Beziehungs)adjektiv ist. Entsprechendes gilt für jablonevye, das in jablonevyj formal-paradigmatisch enthalten ist.

Die Feststellung einer Ableitungsrichtung zwischen jabloko und jablonja stößt auf Schwierigkeiten, die TIX etwa dadurch umgeht, daß er für jedes Wort eine eigene Wortfamilie aufstellt. Diese Lösung ist schon deshalb unannehmbar, weil sie jabloko und jablonja nicht anders als die zahlreichen Fälle einer rein lautlichen Übereinstimmung bedeutungsfremder Wörter behandelt. (Es ist unverständlich, daß TIX daneben stopa 'Stapel' und stopa 'großer Pokal, Humpen, Becher', skovoroda 'Pfanne' und skovorodit' 'nach Art des Schwalbenschwanzes zuhauen' [zu skovorodnja, skovorodnik, skovoroden' 'Schwalbenschwanz'] zu einer Wortfamilie zusammenfaßt.)

Eine Entscheidung in dieser Frage ist nur in Zusammenhang mit dem allgemeineren Problem der Behandlung der Baum-Frucht-Polysemie in unserer Kategorie möglich. Die Bedeutungsvariante 'Frucht' ist unseres Erachtens als primär anzusehen; von ihr sind die Pflanzenbezeichnungen abgeleitet. Dafür spricht die Existenz von Ableitungen, in denen die Polysemie von gruša oder banan in Richtung auf die Baumbezeichnung aufgelöst wird: gruševina 'Bimbaum' oder banannik 'Bananenbaum'. Es gibt sogar ein (freilich nicht monolexematisches) Wortbildungsmodell, das Frucht- in Baumbezeichnungen umwandelt: die Verbindung des Beziehungsadjektivs mit dem Substantiv derevo: gruševoe derevo, ajvovoe derevo, granatovoe derevo etc. Der umgekehrte Fall, bei dem nach der Pflanze die Frucht bezeichnet wird, tritt viel seltener auf; überdies weisen solche Bezeichnungen meist eine zusätzliche (partitive, singulative) Nuance auf, wie im Beispiel von luk $\rightarrow$ lukovica. All dies spricht dafür, auch im Fall von jabloko/jablonja die Fruchtbezeichnung als primär anzusehen.

2. Das Wortbildungsparadigma von svekla lautet:

sveklovica
sveklovišče
sveklovod
sveklovodstvo
sveklovodíeskıj
sveklokombajn
sveklosaxarnyj
sveklosovxoz
svekolka
svekol'nik
svekol'nyj
podsvekol'nik
sveklokopatel
sveklomojka
sveklopogruzc̈ik




sveklopod-emnik
sveklopriemočnyj
sveklorezka
sveklosemena
sveklosejuščij
sveklosejanie
sveklouborka
svekloutomlenie

Sveklovodstvo wird aus dem Paradigma nicht ausgeschlossen: zwar ist sveklovod in ihm formal enthalten und die Bedeutungsumschreibung 'special'nost' sveklovoda; to, čem zanimaetsja sveklovod' ohne weiteres denkbar; ebenso natürlich (wenn nicht sogar natürlicher) ist es jedoch, sveklovod durch sveklovodstvo zu erklären. Sveklovodčeskij steht zu sveklovodstvo, von dem es dem Inhalt nach abgeleitet ist, in einem formalen Intersektionsverhältnis und wird deshalb ebenfalls in das Wortbildungsparadigma von svekla aufgenommen. Ähnlich ist Fall von sveklovišče: trotz der (wahrscheinlich zu engen) Bedeutungserklärung in BASl und MAS 'sveklovičnoe pole' wird es nicht von sveklovica abgeleitet, da die Bedingung der formalen Inklusion nicht erfüllt ist.

3. Im letzten Beispiel wurde sveklouboroćnyj aus dem Wortbildungsparadigma von svekla ausgeschlossen, weil es als 'otnosjaščijsja k sveklouborke' zu definieren ist, während umgekehrt sveklouborka kaum als 'sveklouboročnaja dejatel'nost" umschrieben werden kann. Aus ähnlichen Gründen werden Adjektive wie avtosboroćnyj (avtosborka), avtodorożnyj (avtodoroga), avtozavodskoj (avtozavod), avtozapravočnyj (avtozapravka) aus dem Wortbildungsparadigma von avtomobil/avto ausgeschlossen. Anders liegen die Dinge bei fljagomoečnyj oder kartofelesortirovočnyj, wo das entsprechende Substantiv konkrete Bedeutung hat: fljagomojka ist als 'fljagomoečnaja mašina', kartofelesortirovka als 'kartofelesortirovočnaja mašina' zu definieren.

4. Solovejčik, kukušečka, kastrjulečka werden in das Wortbildungsparadigma von solovej, kukuška und kastrjulja aufgenommen, obwohl solovejko, kukušica und kastrjul'ka in ihnen formal enthalten sind; wir gehen nämlich in solchen Fällen stets von der prinzipiellen Umkehrbarkeit der Bedeutungsumschreibungen aus: falls man die Bedeutungsumschreibung von solovejčik durch solovejko als legitim ansieht, dann auch die von solovejko durch solovejčik; etc.

5. Butyl' ist in Ableitungen wie sobutyl'nik oder butylomeečnyj formal enthalten, gilt jedoch nicht als deren Ableitungsbasis, weil diese Wörter natürlich nicht unter Rückgriff auf butyl definiert werden können. Man beachte, daß in diesem und vielen ähnlichen Fällen die Beziehung der betreffenden Ableitung zum primären Wort der - ganzen Wortfamilie nicht in Betracht kommt: für die Einfügung von sobutyl'nik und sobutyl'ničat' in das Wortbildungsparadigma von butylka spielt es nach den aufge- 
stellten Regeln keine Rolle, daß diese Wörter am natürlichsten nicht durch butylka, sondern durch vypivat', pjanstvovat' definiert werden können. Ebensowenig verhindert die formale Intersektionsbeziehung zwischen jabloko und jablonovka die Aufnahme des letzteren in das Wortbildungsparadigma von jabloko.

6. Synonyme Ableitungen werden auch dort, wo zwischen ihnen echte Inklusionsbeziehungen bestehen, ohne Ausnahme in das Wortbildungsparadigma des Ausgangswortes aufgenommen: zwar ist golubjatnja in golubjatnik formal enthalten und 'to že, čto golubjatnja' eine gültige Bedeutungsumschreibung von golubjatnik, doch kann man auch umgekehrt golubjatnja mit 'to że, čto golubjatnik' erklären.

7. Žarovnja ist von żarit' abgeleitet und wird deshalb nach Fall 2 behandelt. Die Aufnahme der beiden Ableitungen zarovennyj und zaroven'ka in dessen Wortbildungsparadigma verläuft problemlos, da žarovnja in ihnen sowohl formal als auch inhaltlich enthalten ist.

8. Posuda ist (gegen TIX) mit sudno zu einer Wortfamilie zusammenzufassen (man erinnere sich an unsere Vereinbarung, bei der Zusammenfassung von Lexemen zu Wortfamilien bei der Inhaltsseite relativ großzügig vorzugehen); dann ist sudno als primär anzusehen, und bei der Konstruktion des Wortbildungsparadigmas von posuda muß man nach Fall 2 vorgehen. Dies führt dazu, daß neben posudina auch posudinka in das Wortbildungsparadigma aufgenommen werden muß, was angesichts der Verselbständigung von -inka als Singulativsuffix sicher nicht unplausibel ist. Man beachte auch, daß posudina in MAS über sosud definiert wird; US̈A definiert jedoch posudina als 'to že, čto posuda' und gibt uns damit die Möglichkeit, posudina überhaupt in das Wortbildungsparadigma von posuda aufzunehmen (bei der Konstruktion von Wortbildungsparadigmen wollen wir es ja mit den Definitionen viel genauer nehmen).

Wir wollen nicht verschweigen, daß es auch eine Anzahl von Fällen gibt, in denen unsere Regeln zu unbefriedigenden Ergebnissen führen:

1. Das Kriterium der formalen Inklusion führt dazu, daß zwar avtomobilistka wegen avtomobilist, nicht jedoch jabloćnica wegen jabločnik aus dem Wortbildungsparadigma des jeweiligen Grundwortes ausgeschlossen wird. Oder ist die inhaltliche Beziehung in diesen Fällen als symmetrisch anzusehen?

2. In der Wortfamilie von čaša bzw. caška kann man entweder das erste Wort oder das zweite als primär ansehen. Beide Entscheidungen sind legitim und sinnvoll: die formalen Verhältnisse sprechen zwar dafür, čaša als primär anzusehen, doch in der Struktur der Kategorie Geschirr und Besteck nimmt čaška heute sicher eine zentralere Stellung ein und dürfte deshalb als kognitiver Bezugspunkt für die Einordnung von čaša dienen. Geht man nun von čaška aus, so muß man außer čaša auch 
die Ableitungen čašnik und čašničat in sein Wortbildungsparadigma aufnehmen, da diese (ähnlich wie sobutyl'nik und sobutyl'ničat') nur zur Not unter Zuhilfenahme von čaša umschrieben werden können.

3. Bei den abgeleiteten Konkreta maslina und šipovnik, die nach Fall 2 behandelt werden müssen, werden Ableitungen wie masličnyj und šipovennyj aus dem Wortbildungsparadigma ausgeschlossen, da das Grundwort in ihnen nicht formal enthalten ist.

4. Voron und vorona müssen zu einer Wortfamilie zusammengefaßt werden. (Es gibt sogar Sprecher des Russischen, die vorona für das Weibchen eines voron halten.) Falls man nun das Wortbildungsparadigma von voron konstruiert, so ist voronenok und voron'e nach Fall 1.2.1.2 daraus zu tilgen, wobei vorona als Zwischenglied (Wort B) auftritt; Entsprechendes gilt für das Wortbildungsparadigma von vorona mit voron als $\mathrm{Zwischenglied.} \mathrm{Hier} \mathrm{ist} \mathrm{es} \mathrm{gerade} \mathrm{die} \mathrm{Tatsache,} \mathrm{daß} \mathrm{voron'e} \mathrm{und} \mathrm{voronenok}$ von beiden Wörtern abgeleitet sind, die dazu führt, daß sie aus beiden Wortbildungsparadigmen gestrichen werden. Wir können diesen Fehler leicht beheben, indem wir verlangen, daß im Fall einer solchen doppelten Streichung das entsprechende Wort in beide Wortbildungsparadigmen wieder aufzunehmen ist.

Trotz dieser unerwünschten Nebeneffekte erlauben uns unsere Regeln, in einer Vielzahl strittiger Fälle die Entscheidung über die Ableitungsrichtung auf eine einfache und elegante Weise zu umgehen. Zwar werden dadurch einige Lexeme, die man lieber auf einer höheren Stufe vom Grundwort abgeleitet sehen möchte, direkt an dieses angeschlossen: wir erhalten verzweigte Strukturen, wo auch eine Kettenstruktur ins Auge gefaßt werden könnte. Dies ist jedoch das kleinere Übel, da es sich ja bei solchen Zuordnungen um kein unzutreffendes, sondern lediglich un ein unvollständiges Ergebnis handelt: wegen der Transitivität der Ableitungsbeziehung gehen ja auch alle Ableitungen höherer Ableitungsstufen mittelbar auf das Grundwort zurück. 


\section{PARADIGMATISCHE WORTBILDUNGSANALYSE: THEORETISCHE FRAGEN}

Dieses Kapitel ist der Methode der paradigmatischen Wortbildungsanalyse gewidmet. Als erstes stellen wir drei allgemeine Anforderungen auf, denen die paradigmatische Wortbildungsanalyse genügen muß (3.1). Daraufhin begründen wir, warum wir die Wortbildungskategorie als Ganzes und nicht die semantischen Komponenten (Seme), in die man sie zerlegen könnte, als Grundeinheit unserer Analyse gewählt haben (3.2). Das zentrale Einteilungskriterium für den Bereich der desubstantivischen Wortbildung -- der Gegensatz zwischen metaphorischen und metonymischen Ableitungen -- wird in einem eigenen Abschnitt behandelt (3.3). Der nächste Abschnitt enthält einige Überlegungen zur Ermittlung der Wortbildungsbedeutung von Beziehungsadjektiven (3.4). Als letztes wollen wir darlegen, wie wir mit der Polysemie der ableitenden (3.5.1) bzw. der abgeleiteten (3.5.2) Wörter umgehen wollen.

\subsection{Analyseprinzipien}

Wie in der Einleitung bereits angedeutet, gehen wir bei unserer Analyse von den semantischen und formalen Oppositionen aus, die wir in den konkreten Wortbildungsparadigmen vorfinden, und versuchen, mit deren Hilfe jene Wortbildungskategorien zu ermitteln, die den einzelnen Ableitungen zugrundeliegen. Unsere Annahme ist dabei, daß alle konkreten Wortbildungsparadigmen einer bestimmten Gruppe von Wörtern Realisationen desselben abstrakten Wortbildungsparadigmas sind, indem alle Ableitungen, die in ihnen vorkommen, aus einem bestimmten Inventar von Wortbildungskategorien gewählt werden müssen. Die Aufstellung der einzelnen Wortbildungskategorien muß folgenden Prinzipien genügen:

A. Vollständigkeitsprinzip: Es müssen alle Wortbildungskategorien eingeführt werden, die zur Differenzierung nichtsynonymer Ableitungen eines Wortbildungsparadigmas erforderlich sind (cf. REVZIN 1967: 259).

Das Vollständigkeitsprinzip verlangt, daß nichtsynonyme Ableitungen desselben Wortbildungsparadigmas stets unterschiedlichen Wortbildungskategorien zugeordnet werden. Es darf nicht dadurch verwässert werden, daß man bestimmte Oppositionen aus semantischen Erwägungen heraus als wortbildungsirrelevant einstuft, wie es DEBATY-LUCA in seiner bereits erwähnten Arbeit durch die Einführung des Begriffs der "Diskretismen" tut (1986: 308sq., cf. 274-280). Strenggenommen dürfen wir also nur Ableitungen, bei denen absolute Synonymie vorliegt (tykvoobraznyj und tykvopodobnyj 'kürbisförmig' čaševidnyj und čašeobraznyj 'tassenförmig') zu ein und derselben Wortbildungskategorie zusammenfassen.

Das Vollständigkeitsprinzip soll für zwei Klassen von Ableitungen nicht gelten: a) für Komposita und b) für natural kinds, d.h. für Tier- und Pflanzenbezeichnungen. Ableitungen dieser beiden Klassen sollen, soweit dies möglich ist, mit den Wort- 
bildungskategorien, die sich aus anderen Oppositionen ergeben, voneinander abgegrenzt werden; die Einführung eigener Kategorien zu ihrer Differenzierung soll erlaubt, jedoch nicht zwingend vorgeschrieben sein. Oppositionen wie

kartofeleterka 'Kartoffelreibe' :: kartofelečistka 'Kartoffelschälmaschine' (zwei Komposita)

svekol'nik 'Rübenkraut, Blätter der Rübe' :: sveklosemena 'Rübensaatgut' (suffixale Ableitung -- Kompositum)

oder auch

orlan 'Haliaëtus' :: podorlik 'Aquila clanga' (zwei natural kinds)

limonka 'zitronenförmige Handgranate' :: limonnik 'Citrus medica' (Artefakt -natural kind)

brauchen demnach nicht immer zur Aufstellung einer eigenen Wortbildungskategorie für jedes der beiden Mitglieder zu führen: wir können (und werden) kartofeleterka und kartofelečistka, svekol'nik und sveklosemena, orlan und podorlik, limonka und limonnik derselben Wortbildungskategorie zuordnen, ohne das Vollständigkeitsprinzip zu verletzen.

Diese Ausnahme muß für jede der beiden Klassen, die sie betrifft, getrennt begründet werden:

Ad a): Bei den Komposita würde das Vollständigkeitsprinzip dazu führen, daß wir schließlich nicht die Struktur von Wortbildungsparadigmen, sondern die lexikalische Semantik der Zweitglieder beschreiben würden (man denke den Gegensatz zwischen 'Sowchose' und 'Farm' in pticesovxoz vs. pticeferma oder zwischen 'waschen' und 'schneiden' in sveklomojka vs. sveklorezka)'. Indem wir sie vom Vollständigkeitsprinzip ausnehmen, gleichzeitig jedoch in das Wortbildungsparadigma einordnen, gelingt es uns, sowohl der funktionalen Äquivalenz zwischen Komposita und affixalen Ableitungen als auch der semantischen Sonderstellung der Komposita Rechnung zu tragen.

' Unsere Arbeit würde dadurch demselben Vorwurf ausgesetzt, den ŻEMSKAJA 1992: 29sq. gegen JACHNOW 1980 erhebt. Wir weisen bei dieser Gelegenheit darauf hin, daß der paradigmatische Gesichtspunkt in JACHNOWs Arbeiten gänzlich ausgeklammert wird; cf. JACHNOW 1980: 33, wo die Diskussion der "Wortnestverhältnisse" mit der Begründung umgangen wird, daß sie zu "Fragen der Semantik der lexikalischen Basen" hinführe. Das Gegenteil ist der Fall: nur der Vergleich mit den anderen Mitgliedern der Wortfamilie erlaubt es, von der gemeinsamen lexikalischen Bedeutung der Ableitungsbasis zu abstrahieren. 
Ad b): Wir hatten bereits in 1.1 die Gelegenheit, darauf hinzuweisen, daß in der Inhaltsstruktur von natural kinds die enzyklopädische Komponente mit der lexikalischen sehr eng verwoben ist. Das bedeutet, daß die Inhaltsseite von Tier- und Pflanzenbezeichnungen eine Plastizität besitzt, die die Systematisierung nach semantischen Oppositionen von vornherein zum Scheitern verurteilt: die Eigenschaften, die etwa einen orlan von einem podorlik unterscheiden, bilden eine prinzipiell offene Liste; keine unter ihnen darf eine zentrale Stellung beanspruchen, und keine ist dergestalt im Wortbildungssystem verankert, daß sie in anderen Wortbildungsparadigmen wiederkehren würde. Es ist deshalb in den meisten Fällen witzlos, eigene Wortbildungskategorien zur Unterscheidung von natural kinds aufzustellen. Dies gilt allerdings nicht für jene Fälle, in denen ein natural kind durch eine zusätzliche semantische Komponente wie 'weiblich' oder 'jung' modifiziert wird: Ableitungen wie voronixa 'Rabenweibchen' und voronenok 'Rabenjunges' sind keine reinen natural kinds, sondern hybride Gebilde mit einer stabilen sprachlichen Bedeutungskomponente (cf. SCHWARTZ. 1980: 190); sie werden deshalb vom Vollständigkeitsprinzip nicht ausgenommen. Der hybride Charakter solcher Kombinationen eines natural kind mit einem nominal kind zeigt sich auch darin, daß sie zwar Hyponyme, aber keine Taxonyme des Ausgangsbegriffes sind (der erste Terminus geht bekanntlich auf J. LYONS zurück, den zweiten übernehmen wir von CRUSE 1986:137): der Umfang von orlica 'Adlerweibchen' ist im Umfang von orel 'Adler' enthalten; trotzdem stellt dieses Wort keine Art (vid) der Gattung (rod) der Adler (orly) dar wie etwa berkut 'Aquila regia' oder podorlik 'Aquila clanga'. Mit gewissen Vorbehalten kann man sagen, daß ein echtes Taxonym eines natural kind selber ein echtes natural kind sein muß (cf. CRUSE 1986: 140sq.).

B. Ökonomieprinzip: Je zwei Wortbildungskategorien müssen in mindestens einem konkreten Wortbildungsparadigma durch formal unterschiedliche Ableitungen vertreten sein.

Das Ökonomieprinzip verbietet z.B., dem semantischen Unterschied zwischen den Ableitungen

malinnik 'Liebhaber von Himbeeren'

und vinogradar' 'Winzer, Weinbauer'

dadurch Rechnung zu tragen, daß wir jede von ihnen einer eigenen Wortbildungskategorie zuschlagen: der semantische Gegensatz zwischen 'mögen' und 'anbauen' wird nämlich in unserem Korpus in keinem einzigen Wortbildungsparadigma durch formal unterschiedliche Ableitungen zum Ausdruck gebracht.

An diesem letzten Beispiel wird deutlich, daß jede neue Ableitung die Gewichte innerhalb des Wortbildungssystems (und damit auch die Grenze zwischen Wortbildung und Lexikologie) verschieben kann: der Wortbildungstyp der Personenbe- 
zeichnungen auf -nik mit der Wortbildungsbedeutung 'Liebhaber von...' ist besonders in der razgovornaja rec 'so produktiv, daß vinogradnik mit der Bedeutung 'Liebhaber von Weintrauben; jemand, der besonders gem Weintrauben ißt' von vinograd ohne weiteres abgeleitet werden könnte, wenn das Wort nicht bereits in der Bedeutung 'Weinberg' lexikalisiert wäre (cf. ZEMSKAJA 1992: 98). Fügt man vinogradnik 'Liebhaber von Weintrauben; jemand, der besonders gern Weintrauben ißt' dem Wortbildungsparadigma von vinograd hinzu, so verändert sich der Status der Opposition zwischen 'mögen' und 'anbauen', da sie nunmehr zumindest im Wortbildungsparadigma von vinograd als relevant anzusehen ist (vinogradnik :: vinogradar). Die paradigmatische Wortbildungsanalyse, die für verschiedene Korpora verschiedene Wortbildungskategorien liefert, statt diese apriorisch festzusetzen (s. die Einleitung), spiegelt an diesem Punkt nur die Beweglichkeit der Grenze zwischen Lexikologie und Wortbildung wider.

Die Kehrseite des Ökonomieprinzips ist, daß Wortbildungskategorien, deren Vertreter in keinem einzigen Wortbildungsparadigma zueinander in formaler Opposition stehen, ungeachtet aller semantischen Unterschiede miteinander verschmolzen werden müssen: so müßte man z.B. im abstrakten Wortbildungsparadigma der Kategorie pticy (Vögel) die traditionellen Kategorien der Kollektiva (voron'e, koršun'e), der Augmentativa (utišča) und der Pejorativa (kurčonka) zu einer Kategorie zusammenfassen, da in unserem Korpus kein einziges Wortbildungsparadigma zugleich ein Augmentativum, ein Kollektivum und ein Pejorativum aufweist.

Auch das Ökonomieprinzip muß eine Einschränkung erfahren: es soll nur für Ableitungen gelten, die derselben Wortart angehören. In der Praxis bedeutet dies, daß wir die substantivische, die adjektivische und, falls vorhanden, die verbale und adverbiale Zone unserer Wortbildungsparadigmen getrennt analysieren werden; wir nehmen also an, daß alle Ableitungen einer Wortbildungskategorie in ihrer Wortartzugehörigkeit übereinstimmen. Damit soll nicht geleugnet werden, daß es zwischen Ableitungen verschiedener Wortarten bedeutende semantische Übereinstimmungen geben kann: die Inhaltsseite eines Verbs wie štoporit' 'trudeln' steht dem Adverb štoporom 'spiralförmig, schraubenförmig' viel näher als Verben wie goršećničat 'das Töpferhandwerk treiben' oder sobutyl'ničat' 'zechen' (cf. petušit'sja 'hitzig sein, sich wie ein Hahn brüsten' und das ihm entsprechende Adverb petuškom); doch hieße es jede Verbindung zwischen Formen- und Wortbildung leugnen, wollte man dies zum Anlaß nehmen, wortartenübergreifende Wortbildungskategorien zu bilden.

C. Natürlichkeitsprinzip: Die aufgestellten Wortbildungskategorien müssen eine natürliche semantische Interpretation zulassen.

Das Natürlichkeitsprinzip verbietet es z.B., das Augmentativum arbuzišče (zu arbuz 'Wassermelone') mit anderen Ableitungen auf -išče wie repišče 'Rübenfeld' oder 
kartofljanišče '(abgeemtetes) Kartoffelfeld' zu einer Wortbildungskategorie zusammenzufassen -- auch wenn durch eine solche Entscheidung eine Verletzung des Ökonomieprinzips vermieden wird (arbuzišče ist in den Wortbildungsparadigmen der Kategorie ovošč [Gemüse] das einzige Augmentativum; es kommt nur in der Opposition arbuzik :: arbuzišče vor).

Wie man unschwer sieht, steht die Forderung nach Natürlichkeit der Beschreibung zu den rein formalen Forderungen nach Ökonomie und Vollständigkeit in einem gewissen Widerspruch; sie ist auch insofern von ihnen verschieden, als sie Abstufungen zuläßt (eine Wortbildungskategorie kann mehr oder weniger natürlich sein).

Man darf sich fragen, ob die Eigenschaften natürlicher Kategorien, wie sie in Kap. 1.2 skizziert worden sind, auf Wortbildungskategorien übertragen werden können. Besteht die Natürlichkeit von Wortbildungskategorien darin, daß sie mehr oder weniger zentrale Mitglieder besitzen und daß die Übergänge zwischen ihnen fließend sind? Gibt es auch bei ihnen so etwas wie eine Basisebene -- eine mittlere Abstraktionsstufe, auf der sie am leichtesten voneinander abgegrenzt werden können? Muß man vielleicht nach dem Vorbild von LAKOFF 1987 die Wortbildungskategorien als radiale Strukturen auffassen, in denen ein prototypischer Kern nach verschiedenen Richtungen hin erweitert wird? So reizvoll diese Perspektive hier scheinen mag, wir wollen doch darauf verzichten, das Prinzip der Natürlichkeit auf diese Weise zu dehnen. Unsere Wortbildungskategorien besitzen ja keine feste Ausdrucksseite, die sie zusammenhalten könnte; wird ihr Zusammenhalt auch auf der Inhaltsseite gelokkert, so gibt es nichts mehr, was deren Grenzen definieren könnte, und wir könnten durch sukzessive Modifikationen einer abstrakten Kernstruktur fast jede Ableitung mit jeder anderen zu ein und derselben Wortbildungskategorie verschmelzen.

Wir schließen dieses Kapitel mit der Bemerkung, daß die Prinzipien der Vollständigkeit, der Ökonomie und der Natürlichkeit lediglich die Anforderungen umreißen, denen das Ergebnis der Wortbildungsanalyse genügen muß, jedoch nichts über den Weg aussagen, der zu diesem Ergebnis führt; einen Algorithmus zur Aufstellung abstrakter Wortbildungsparadigmen kann man aus ihnen nicht ableiten.

\subsection{Wortbildungskategorien und Komponentenanalyse}

Wir sind bislang stets davon ausgegangen, daß die Wortbildungskategorien die letzten Einheiten der Inhaltsebene sind, mit denen das Wortbildungssystem einer Sprache operiert. Viele Oppositionen in den Wortbildungsparadigmen legen jedoch nahe, auch diese Einheiten in Teileinheiten zu zerlegen. Man betrachte dazu Paare wie

repišče 'Rübenfeld' :: repnjak 'Kraut, Blätter der Rübenpflanze' 
sveklovišče 'Zuckerrübenfeld' :: svekol'nik 'Rübensuppe'

goroxovišče '(abgeerntetes) Erbsenfeld' :: goroxovina 'der Erbsenstengel mit Blättem, das Erbsenstroh'.

Es bietet sich an, ein abstraktes Merkmal wie $[ \pm \operatorname{lok}(\mathrm{al})]$ zu postulieren, um den gemeinsamen semantischen Kern der Ableitungen der linken Spalte (alles Bezeichnungen für Felder, auf denen die betreffende Pflanze angebaut wurde) zum Ausdruck zu bringen und sie von den Ableitungen der rechten Spalte zu differenzieren. Entsprechend könnte man durch ein Merkmal wie [ \pm art(ifiziell)] die beiden Ableitungen kotlovan 'Baugrube' und kotlovina 'Kesselschlucht' voneinander abgrenzen; etc. Eine solche Notation würde zudem erlauben, die semantische Verwandtschaft zwischen Ableitungen verschiedener Wortbildungskategorien, die sonst unvermittelt nebeneinander stünden, direkt zum Ausdruck zu bríngen: kartofljanišče '(abgeerntetes) Kartoffelfeld' und kartofelexranilišče 'Kartoffelmiete', gusjatnik 'Gänsestall' und gusjatnica 'Gefäß zum Braten von Gänsen' würden z.B. alle das Merkmal [+loc] teilen, das Ableitungen wie kartofelevod 'Spezialist für Kartoffelanbau' und gusevod 'Gänsezüchter' fehlt.

Ganz neu wäre ja ein solches Verfahren im Bereich der Wortbildungslehre nicht; allerdings waren es bislang vorwiegend präfixale Ableitungen, die im Gefolge der berühmten Untersuchung von R. JAKOBSON zum Kasussystem des Russischen nach binären Merkmalen klassifiziert wurden (VAN SCHOONEVELD 1978, FIIIER 1975, GALLANT 1979). Am liebsten setzt man solche Merkmale für die Randbereiche des Wortbildungssystems an, die mit Hilfe der syntaktisch definierten Wortbildungskategorien nicht mehr erfaßt werden können: cf. etwa die Unterscheidung von kategorialen und sekundären Merkmalen bei BuZÁsSYOVÁ 1974: 51-71 oder den Begriff der qualifikativen Merkmale bei RevzinA 1969: 19sqq. Die Analyse'von Wortbildungsbedeutungen durch distinktive semantische Merkmale ist allerdings in letzter Zeit auf heftige Kritik gestoßen, cf. JANDA 1986: 27-32 und DEBATY-LUCA, der dieser Frage ein eigenes Kapitel seiner Arbeit gewidmet hat (DEBATY-LUCA 1986: 281-292).

Wir wollen die Argumente der Gegner der Komponentenanalyse hier nicht wiederholen, sondern an einem Beispiel aus unserem eigenen Material aufzeigen, warum die Darstellung der Inhaltsseite von Wortbildungskategorien mit Hilfe elementarer semantischer Merkmale nur einen scheinbaren Gewinn mit sich bringt. Man betrachte dazu die Wortbildungskategorie, der Ableitungen wie abrikosovka, klubnikovka, limonovka etc. angehören -- Bezeichnungen für hausgemachte Branntweine und Liqueure ('Aprikosenliqueur', 'Erdbeerbranntwein', 'Zitronenbranntwein'). Die Wortbildungsbedeutung dieser Ableitungen könnte man ansatzweise in folgende Elemente zerlegen: 
Als erstes bräuchte man ein Merkmal, um alle Ableitungen, denen eine metonymische Beziehung zugrundeliegt (limonarij 'Pflanzstätte für Zitronen'), von Ableitungen abzugrenzen, denen eine metaphorische Beziehung zugrundeliegt (limonka 'zitronenförmige Handgranate'); in der Kategorie der metonymischen Ableitungen müßte man dann durch ein eigenes Merkmal Personen- von Gegenstandsbezeichnungen voneinander unterscheiden (vinogradar' 'Winzer' vs. vinogradnik 'Weinberg'); aus den Gegenstandsbezeichnungen könnte man Bezeichnungen für Nahrungsmittel (limonad 'Limonade') ausgliedern, um schließlich durch ein letztes, sehr spezifisches Merkmal die Opposition zwischen limonad und limonovka zum Ausdruck zu bringen. Kürzt man diese vier Merkmale als $[ \pm \mathrm{mtn}, \pm$ hum, \pm ali, \pm vin] ab, so erhält man für limonovka die Kombination [+mtn, -hum, +ali, +vin], für limonad die Kombination [+mtn, -hum, +ali, -vin], für limonarij und vinogradnik die Kombination [+mtn, -hum, -ali, -vin] und für vinogradar die Kombination [+mtn, +hum, -ali, -vin].

Geht man nun andere Merkmalskombinationen durch, so überzeugt man sich sehr bald, daß die meisten unter ihnen nicht nur in den Wortbildungsparadigmen des Russischen nicht belegt, sondern auch sachlich unmöglich sind. Keine Ableitung kann zugleich [+hum, +ali] (Bezeichung für eine Person, die als Nahrungsmittel dient) oder [-ali, +vin] sein (ein Branntwein, der kein Nahrungsmittel ist). Implikationsbeziehungen dieser Art können durch keine auch noch so abstrakte Analyse vermieden werden; sie sind ein Anzeichen dafür, daß die Zerlegung der Wortbildungskategorien in elementare Einheiten nur scheinbar stattgefunden hat: ein Merkmal wie [+vin] enthält genauso viel semantische Information wie die entsprechende Wortbildungskategorie. Den Zusammenhang zwischen unseren Wortbildungsparadigmen hat man sich als ein Baumdiagramm vorzustellen, in dem jede Verzweigung ein eigenes Oppositionssystem eröffnet: Personenbezeichnungen können nach Geschlecht, Bezeichnungen für Räumlichkeiten nach den Tätigkeiten, die in ihnen stattfinden, Bezeichnungen für Nahrungsmittel nach Alkoholgehalt bzw. Art der Herstellung differenziert werden. Eine Übertragung der Gliederungsprinzipien von einem Zweig dieses Diagramms auf einen anderen ist selten möglich; die Einführung elementarer semantischer Merkmale in die Analyse der Wortbildungskategorien führt -- um die Termini von ZALIZNJAK 1967: 6-9 zu benutzen -- nicht zu einer universellen, sondern zu einer baumförmigen Klassifikation. Rein technisch ist es zwar immer möglich, eine baumförmige Klassifikation durch Uminterpretation der Merkmale in eine universelle umzuwandeln; das ergibt aber massive Verletzungen des Natürlichkeitsprinzips. Aber auch dort, wo einzelne Merkmale auf natürliche Weise von einer Ableitungsgruppe auf eine andere übertragen werden können, braucht das Ergebnis nicht unbedingt sinnvoll zu sein: nichts kann nämlich gewährleisten, daß eine Unterscheidung, die z.B. im Bereich der Personenbezeichnungen durch eine formale Opposition zum Ausdruck gebracht wird, dieselbe Relevanz auch im Bereich der Gegenstandsbezeichnungen aufweist. Eine Zerlegung in elementare semantische Merkmale hätte hier eine Überdifferenzierung der Wortbildungskategorien zur Folge. 
Wir schließen also, daß nicht elementare semantische Merkmale, sondern Wortbildungskategorien jene Einheiten sind, mit denen das Wortbildungssystem operiert; ihre semantische Zerlegung ist vom Standpunkt der Wortbildung aus irrelevant -- sie stellt bestenfalls ein (recht unhandliches) Mittel dar, um die Wortbildungskategorien aufgrund ihrer semantischen Verwandtschaft in übergreifende Gruppen einzuteilen. Diese letztere Aufgabe ist allerdings nicht sinnlos; in der einen oder anderen Form kehrt sie in vielen Wortbildungsarbeiten wieder. Wir werden deshalb im nächsten Kapitel das wichtigste Kriterium behandeln, nach dem im Bereich der desubstantivischen Ableitungen eine solche Einteilung vorgenommen werden kann: den Gegensatz zwischen den metaphorischen und den metonymischen Ableitungsbeziehungen.

\subsection{Metaphorische und metonymische Ableitungsbeziehungen}

Bei unserer Diskussion der Semantik konkreter Substantive haben wir auf die Rolle hingewiesen, die metaphorische und metonymische Assoziationen (Ähnlichkeits- und Kontiguitätsassoziationen) für die Strukturierung natürlicher Kategorien spielen. Wir wollen nun zeigen, daß im Bereich der desubstantivischen Wortbildung dieselben Mechanismen wirksam sind. Dazu müssen wir zunächst das Problem der Metapher und der Metonymie in abstrakterer Form behandeln und die rein psychologische Betrachtungsweise verlassen; wir wollen deshalb fortan statt von metaphorischen und metonymischen Assoziationen lieber von metaphorischen und metonymischen Beziehungen sprechen. ${ }^{2}$

Unseren Ausgangspunkt bildet die grundlegende Dualität von Standpunkt (Subjekt) und Gegenstand (Objekt); wir definieren nun:

- Aspekte ein und desselben Gegenstandes, der von verschiedenen Standpunkten aus betrachtet wird, stehen zueinander in metaphorischer Beziehung;

- verschiedene Gegenstände, die von ein und demselben Standpunkt aus betrachtet werden, stehen zueinander in metonymischer Beziehung.

${ }^{2}$ Die folgenden Überlegungen wurden bewußt ganz knapp gehalten; für eine ausführlichere Darstellung s. KAKRIDIS (im Druck). Eine umfassende Diskussion der Metapher, die sich ja in den letzten zwei Jahrzehnten zu einer Art Modethema entwickelt hat, würde den Rahmen dieser Arbeit sprengen; s. dazu die Sammelbände ORTONY (ed.) 1993, DiRVEN/PAPROTTE (eds.) 1985, JOHNSON (ed.) 1981. 
Beispiele:

1. Alle Projektionen eines festen Körpers (z.B. eines Würfels) stehen zueinander in metaphorischer Beziehung; die Teile, die an ihm gleichzeitig wahrgenommen werden können (Kanten, Seiten etc.) stehen zueinander in metonymischer Beziehung.

2. Vexierbilder wie das der Ente, die auch als Hase gesehen werden kann (nach WITTGENSTEIN 1984: 519-522); oder der Vase, deren Konturen auch als zwei menschliche Profile in Gegenüberstellung gesehen werden können (nach SCHÖN 1993): die Bilder, die sich bei jeder der beiden Sichtweisen ergeben, stehen zueinander in metaphorischer Beziehung; Kopf und Ohren des Hasen, Fuß und Rand der Vase etc. stehen zueinander in metonymischer Beziehung.

3. Soziale Systeme können in der Sprache der Biologie (als menschlicher Körper), der Mechanik (als Uhrwerk) oder der Schiffahrt (als Schiff) beschrieben werden; die Beschreibungen, die sich jeweils ergeben, stehen zueinander in metaphorischer Beziehung, sowohl als Ganze als auch in ihren Bestandteilen (z.B. Kopf :: Kapitän); die einzelnen Bestandteile jeder Beschreibung (Kapitän :: Mannschaft, Kopf :: Füße) stehen zueinander in metonymischer Beziehung.

4. Über- und Unterordnungsbeziehungen innerhalb einer Taxinomie erlauben ebenfalls eine metaphorische Deutung: ein und derselbe Gegenstand kann ja z.B. als Blockföte, Flöte, Blas- oder Musikinstrument bezeichnet werden; den Wechsel des Standpunktes kann man sich in diesem Fall als eine allmähliche Entfernung vom Gegenstand vorstellen, bei dem dessen Konturen verschwimmen. (Aristoteles war also durchaus berechtigt, unter den vier Hauptarten der Metapher Übertragungen von der Art auf die Gattung und vice versa aufzuführen; cf. Kap. 1.2.2 dieser Arbeit.)

Im Spannungsfeld von metonymischen und metaphorischen Beziehungen können vier Tropen angesiedelt werden: Metonymie (zu der im weiteren Sinne auch die Synekdoche gehört; zum Verhältnis beider Tropen S. BIRIX 1995:19-23), Metapher (die als Extremfall die Ironie einschließt), Vergleich und Symbol.

Bei der Metonymie tritt ein Gegenstand für einen anderen Gegenstand ein, weil zwischen ihnen eine metonymische Beziehung besteht: ein männlicher Schuh markiert den Eingang zu der Herrentoilette, "zehn Köpfe" steht für "zehn Menschen" etc.; da wir es bei metonymischen Beziehungen stets mit zwei verschiedenen Gegenständen zu tun haben, bereitet die Vorstellung einer Ersetzung (Substitution) keine Schwierigkeiten.

Weitaus größere Hindernisse stellt die Metapher dem Verständnis entgegen. Bei der Metapher haben wir es nicht mit verschiedenen, sondern mit einem einzigen Gegen- 
stand zu tun, der gleichzeitig von mehreren Gesichtspunkten aus betrachtet wird. ${ }^{3}$ Metaphern wie "Lebensfrühling" oder "Lebensabend" liegt die Intuition zugrunde, daß die Entwicklungsphasen biologischer Organismen, die Jahres- und die Tageszeiten nur drei verschiedene Seiten derselben Wirklichkeit sind -- eines bestimmten Rhythmus von Entstehung, Wachstum und Verfall, der für alle Seinsstufen gilt; ein König kann als Hirte seiner Völker bezeichnet werden, weil dies nur zwei verschiedene Ausdrucksweisen für dasselbe Geflecht von Herrschaftsbeziehungen sind. In ihrer einfachsten Form ergibt sich die Metapher aus dem Konflikt zwischen der Einnahme eines Standpunktes und der Einsicht, daß es sich hierbei nur um einen unter vielen möglichen Standpunkten handelt. In dem Maße, in dem diese anderen Standpunkte unspezifiziert bleiben, wird es schwierig, der metaphorischen Ausdrucksweise eine zweite ("wörtliche") an die Seite zu stellen; wir erhalten Metaphem, deren Bedeutung durch keine Paraphrase erschöpft werden kann.

Es liegt nahe, die Spannung, die sich aus dem Konflikt verschiedener Sichtweisen ergibt, dadurch aufzulösen, daß man die Verschiedenheit vom Subjekt ins Objekt verlagert. In diesem Fall hat man es nicht mehr mit einem, sondern mit zwei verschiedenen Gegenständen zu tun, die von einem abstrakten Standpunkt aus zueinander in Beziehung gesetzt werden. Bleibt man auf diese Weise zwischen Metapher und Metonymie auf halbem Wege stehen, so ergibt sich ein Vergleich: Abend, Herbst und Alter sind nicht mehr derselbe Zeitabschnitt, in verschiedenem Maßstab betrachtet; König und Hirte nicht dieselbe Figur in verschiedener Verkleidung; sondern der Abend ist dem Alter ähnlich, und der König erinnert an einen Hirten. Die entsprechende Verschiebung ergibt im Bereich der Rhetorik die Substitutionstheorie der Metapher und ihr Korollar -- die Auffassung von der Metapher als eines verkürzten Vergleiches. Dagegen ist oft eingeworfen worden, daß man mit größerer Berechtigung den Vergleich als eine erweiterte Metapher ansehen kann; dieser Einwurf wird durch die hier gegebenen Definitionen bestätigt.

Wo nicht die Spannkraft der Metapher in Richtung auf die Metonymie abgeschwächt, sondern umgekehrt eine Metonymie mit metaphorischen Obertönen bereichert wird, ergibt sich ein Symbol. ${ }^{+}$Das Standardbeispiel ist das Kreuz -- ursprünglich ein Gegenstand, der als Bestandteil der Passionsgeschichte zum Christentum in metonymischer Beziehung stand, ist es im Laufe der Jahrhunderte mit der christlichen Religion zu einer unauflöslichen Einheit verschmolzen.

3 Sehr passend hat deshalb W. BEDELL STANDFORD die Metapher als "stereoscope of ideas" bezeichnet (1936: 100-105, cf. BERGGREN 1962: 243).

"Nach der bekannten Definition von COLERIDGE: "It [the symbol] always partakes of the Reality which it renders intelligible; and while it enunciates the whole, abides itself as a living part in that Unity, of which it is the representative" (COLERIDGE 1972: 30). 
Folgendes Schema verdeutlicht die Beziehungen zwischen den vier Tropen:

\section{Metapher}

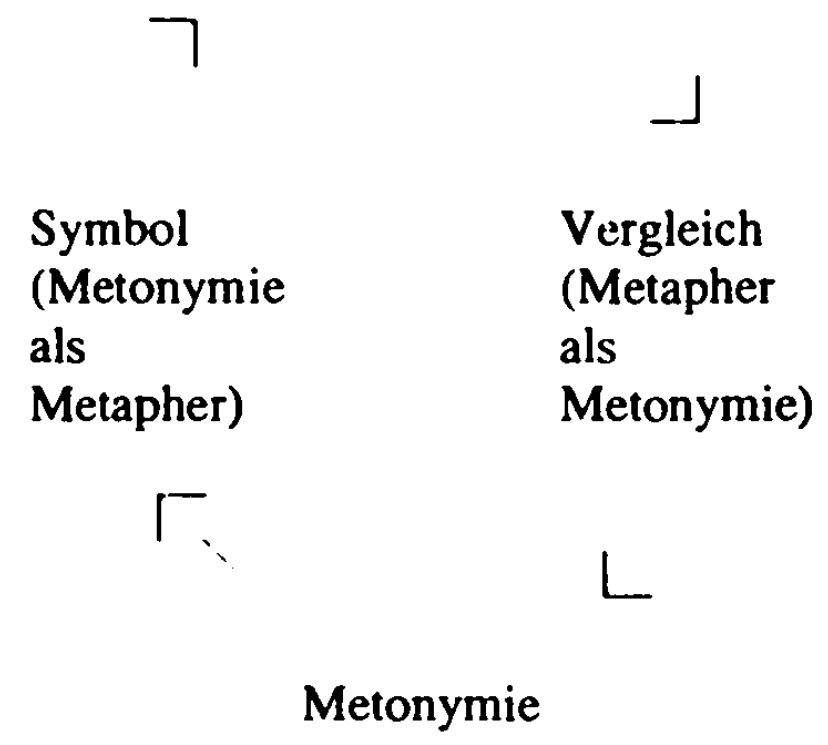

Wir wollen nun zeigen, wie das Zusammenspiel von Metapher und Metonymie im Bereich der desubstantivischen Wortbildung funktioniert. Das abgeleitete Wort benennt ja einen Gegenstand nicht direkt, sondern durch die Feststellung (ustanovlenie) einer bestimmten Beziehung, die ihn mit anderen Gegenständen der Wirklichkeit verbindet (VINOKUR 1959: 421). Die Frage liegt nahe, ob diese Beziehungen metonymischer oder metaphorischer Natur sind. Hier einige Beispiele für Ableitungen, denen eine metonymische Beziehung (Kontiguitätsbeziehung) zugrundeliegt:

toporišče 'Beil-, Axtstiel'

podokonnik 'Fensterbrett'

vinogradar' 'Winzer, Weinbauer'

poxmel'e 'der Zustand, der nach der Trunkenheit (xmel') eintritt: der Katzenjammer'

predgroz'e 'Zeitabschnitt vor einem Gewitter'

kartofelekombajn 'Kartoffelkombine'

sveklosovxoz 'Sowjetgut, in dem Rüben angebaut werden'

kartofelesortirovka 'Kartoffelsortiermaschine'

sveklorezka 'Rübenschneidemaschine'

kartofelemoečnaja mašina 'Kartoffelwaschmaschine'

sveklopriemoćnyj punkt 'Rübenabnahmeplatz'

Trotz aller formalen Verschiedenheit haben diese Ableitungen eine gemeinsame semantische Struktur: der zu benennende Gegenstand (das Nominandum) wird 
zunächst einer allgemeinen Kategorie (der onomasiologischen Basis) zugeordnet und dann mit Hilfe einer zusätzlichen Bestimmung (des onomasiologischen Merkmals) von den übrigen Mitgliedern dieser Kategorie abgegrenzt. Das onomasiologische Merkmal ist wiederum zweigliedrig: es besteht einerseits aus dem Gegenstand, zu dem das Nominandum in Beziehung gesetzt werden soll (dem onomasiologischen Motiv) und andererseits aus der Angabe dieser Beziehung selbst (der onomasiologischen Kopula), die in den angeführten Beispielen stets metonymisch ist (DoKULIL 1962: 29sqq.; DoKULIL 1968: 207sq.).

In Mehrwortbenennungen wie kartofeleuboročnyj kombajn und Komposita wie Kartoffelwaschmaschine sind alle drei Elemente -- die onomasiologische Basis, das onomasiologische Motiv und die onomasiologische Kopula -- durch eigene Kemmorpheme vertreten; in sveklorezka tritt für die onomasiologische Basis das Suffix $-k(a)$ ein; in sveklosovxoz oder vinogradar muß die onomasiologische Kopula erraten werden, in poxmel'e oder podokonnik wird sie durch das Präfix repräsentiert. ${ }^{5} \mathrm{Am}$ metonymischen Grundcharakter der onomasiologischen Kopula ändern diese Variationen der Ausdrucksseite nichts; den Ableitungen liegen folgende Beziehungsarten zugrunde:

- räumliche Kontiguität (podokonnik, toporišče)

- zeitliche Kontiguität (predgroz'e)

- situative Beziehungen (kartofelesortirovka, sveklosovxoz).

Man beachte, daß situative Beziehungen auch Gegenstände, die sich in Raum oder Zeit nicht berühren, miteinander verbinden können; gemeinsame Zugehörigkeit zu einem statischen Ganzen kann diese Brückenfunktion nicht übernehmen: so kann man z.B. die Scheinwerfer eines Autos mit Schiebedach nicht als ?Schiebedachscheinwerfer bezeichnen -- es sei denn, sie sind direkt am Schiebedach angebracht (Kontiguitätsbeziehung) oder mit dem Schiebedach durch ein situatives Band verknüpft (Scheinwerfer, die das Schiebedach beleuchten; Scheinwerfer, die aus alten Schiebedächern hergestellt wurden; etc.); die durch das konkrete Substantiv Auto vermittelte Beziehung reicht jedenfalls nicht aus. ${ }^{6}$

5 Kann das onomasiologische Motiv in der Ableitung fehlen, die onomasiologische Kopula dagegen durch ein Kernmorphem vertreten sein? Dieser Fall liegt in deverbalen Ableitungen wie pugalo 'Vogelscheuche' oder perevozčik 'Fährmann' vor.

${ }^{6}$ Entsprechende Beschränkungen gelten für die Metonymie in der Rhetorik; cf. die anregenden Bemerkungen in GrOUPE 1970: 109. Die integrative Funktion von Handlungsbegriffen kann auch experimentell nachgewiesen werden (HOFFMANN 1986: 139-141). 
Nun lassen sich auch Mehrwortbenennungen finden, die nach demselben dreiteiligen Schema (onomasiologische Basis/Kopula/Motiv) analysiert werden können, bei denen jedoch nicht eine Kontiguitäts-, sondern eine Ähnlichkeitsbeziehung (metaphorische Beziehung) die Funktion der onomasiologischen Kopula übernimmt:

perstnevidnyj xrjašč 'Schildknorpel'

klinovidnye pis'mena 'Keilschrift'

Auch bei Ableitungen, denen metaphorische Beziehungen zugrundeliegen, braucht die onomasiologische Kopula nicht durch ein Kernmorphem vertreten zu sein:

tareločnaja čečevica 'sort čečevicy s krupnymi semenami'

Schließlich kann die onomasiologische Basis durch ein Suffix ersetzt werden:

kotlovina 'Kesselschlucht'

Besonders Deminutiva werden im Russischen regelmäßig dazu benutzt, um Gegenstände zu benennen, die zum Gegenstand, den die Ableitungsbasis bezeichnet, in einer Ähnlichkeitsbeziehung stehen:

gorlyško 'Flaschenhals'

jastrebok 'Jagdflugzeug'

bljudce 'Untertasse, Tellerchen'

Wenn man will, kann man auch solche Ableitungen nach dem Muster von kartofeleuboročnyj kombajn und perstnevidnyj xrjašč analysieren: die onomasiologische Basis, durch das Deminutivsuffix vertreten, wäre allgemein 'Gegenstand', die onomasiologische Kopula die Ähnlichkeitsbeziehung und das onomasiologische Motiv der Gegenstand, den das ableitende Wort bezeichnet: 'Hals', 'Habicht', 'Teller, Schale'. Ja sogar echte Deminutiva lassen sich zur Not in dieses Schema pressen:

bljudečko (Dem. zu bljudo 'Untertasse') 'kleiner Gegenstand, der die Form einer Untertasse hat'

nožik (Dem. zu nož 'Messer') 'kleiner Gegenstand, der einem Messer ähnelt'

Von einem streng logischen Standpunkt aus sind solche Analysen vielleicht möglich, und sie werden immer wieder vorgeschlagen. ${ }^{7}$ Man kann jedoch kaum leugnen, daß

${ }^{7}$ Dies gilt auch für den Vorschlag von HANS MARCHAND, squireling als "dwarf of a squire", "the squire is a petty thing" oder "that miniature is a squire" zu analysieren (MARCHAND 1969: 43sq.; anders noch MARCHAND 1960: 174). Die 
sie das Sprachgefühl verletzen: je tiefer wir in den Kernbereich der metaphorischen Wortbildung vordringen, desto gezwungener wirkt die Anwendung des dreiteiligen Schemas von onomasiologischer Basis, onomasiologischer Kopula und onomasiologischem Motiv. Es ist auch unschwer einzusehen, warum: solange wir bei der semantischen Analyse von Ableitungen wie gorlyško oder nožik die onomasiologische Basis und das onomasiologische Motiv als zwei verschiedene Gegenstände ansehen, die von einem externen Standpunkt aus verknüpft werden, haben wir ja die Vorstellungswelt der metonymischen Wortbildung noch gar nicht verlassen. Um dem metaphorischen Charakter dieser Ableitungen gerecht zu werden, müssen wir eine Kehrtwendung vollziehen und nicht von zwei, sondern von einem einzigen Gegenstand ausgehen, der aus doppelter Perspektive betrachtet wird. So nimmt etwa ein Sprecher, der das Wort gorlyško 'Flaschenhals' gebraucht, zunächst einen bestimmten Standpunkt ein (die Flasche wird als menschlicher Körper gesehen), um ihn durch die Anfügung des Suffixes -yšk(o) in demselben Atemzug wieder anzufechten (die Flasche ist kein richtiger Körper). Daß metaphorische Ableitungen einen solchen Applikationsvorbehalt mit morphologischen Mitteln zum Ausdruck bringen können, wird besonders bei Gegenüberstellungen deutlich (die Hervorhebungen stammen in den folgenden Beispielen immer von mir):

Ne ljudi -- a kakie-to čelovekoobraznye traktory (E. ZAMJATIN, My).

Bumažnye cvety literaturščiny koe-kto prinimaet za podlinnye cvety literatury (Literaturnaja gazeta, zitien nach VINOGRADOVA 1984: 30).

Pisatel' Murav’ev vyšel na ploščadku vagona, otkryl naružnuju dver' i dolgo smotrel na pronosivšujusja mimo poezda zimu.

Ėto byla, požaluj, ne zima, a to, čto nazyvajut "zimkoj". -- pasmurnyj den', kogda poryvami nabegaet syroj veter, vot-vot naćnetsja ottepel' i poletjat s ottajavšix vetok pervye kapli (K. PAUSTOVSKIJ, Roždenie rasskaza).

Tysjačeokij -- kanet $v$ noč,

ne $v$ ètu seren'kuju nočku.

(V. XODASEVIĆ, Iz dnevnika)

Im ersten Beispiel (čelovekoobraznye traktory) liegt keine richtige Metapher, sondern ein expliziter Vergleich vor; Ableitungen dieser Art lassen sich, wie wir gesehen haben, ohne weiteres nach dem Muster metonymischer Ableitungen analysieren. Eine solche Analyse ist bei metaphorischen Ableitungen wie literaturščina und zimka

morphologischen Argumente gegen die Einstufung von evaluativen Suffixen als heads sind bei SCALISE 1988: 233-237 zusammengefaßt; cf. JO NAPOLI/REYNOLDS 1994: 169-175. 
nicht statthaft; sie ist hier aus denselben Gründen verführerisch und in demselben Maße falsch wie die Vergleichstheorie der Metapher.

Als morphologische Exponenten eines Applikationsvorbehaltes sind die Affixe in den metaphorischen Ableitungen den Anführungszeichen vergleichbar, mit denen sie gelegentlich in Kombination auftreten ${ }^{8}$ :

Byl u nas kazennokoštnyj student Operov, skromnyj, očen’ sposobnyj i userdnyj molodoj čelovek, kotoryj podaval vsegda ruku, kak dosku, ne sgibaja pal'cev i ne delaja eju nikakogo dviženija, tak čto šutniki-tovarišči inogda tak že podavali emu ruku i nazyvali èto podavat' ruku "doščečkoj". [...] Zvuk golosa Semenova [...] soveršenno uspokoil nas, i my potoropilis' vyjti vpered i podat' -- ja svoju ruku, Operov svoju doščečku [...] (L. N. TOLSTOJ, Junost').

Metaphorische Ableitungen fordem fermer zu einem Vergleich mit Heckenausdrücken (hedges) heraus; bereits LAKOFF 1972: 196 hat ja Suffixe wie -like oder -ish unter die Heckenausdrücke eingereiht (cf. JOHNSON/LAKOFF 1980: 123sq. sowie den Forschungsüberblick bei KOLDE 1989).

Am reinsten kommt das Wesen der metaphorischen Wortbildung in den expressiven Ableitungen zum Ausdruck: Deminutiva, aber auch Augmentativa, Pejorativa und Meliorativa sind Wortbildungsformen der Uneigentlichkeit, mit denen der Sprecher seine eigene Wortwahl in Frage stellen kann. Entgegen einer weitverbreiteten Ansicht (z.B. VOLEK 1987: 5-34; cf. die Kritik bei BARBARESI/DRESSLER 1994: 29-35, 201 206) ist erhöhte Emotivität kein wesenhafter Bestandteil der Wortbildungsbedeutung von Deminutiva; ihren emotiven Gehalt verdanken solche metaphorischen Ableitungen der Tatsache, daß das Vertrauen der Sprecher in die Macht des Wortes sehr oft dann erschüttert wird, wenn sie darin den Ausdruck des Gefühls vermissen, das sie gerade bewegt. Daß Deminutiva per se keine höhere Emotivität zukommt, machen auch psycholinguistische Experimente deutlich, bei denen die Vpn gebeten werden, Wörter nach bestimmten Parametern einzustufen, von denen man annimmt, daß sie zum emotiven Gewicht des betreffenden Wortes in Beziehung stehen (xorošo/ploxo, sil'no/slabo, żelatel'no/neželatel'no etc.). Der Vergleich der Beurteilung von Deminutiva und ihren Ausgangsbasen zeigt, daß letztere kaum weniger emotiv besetzt sind als erstere; lediglich nach den Parametern ironično/neironično und prenebrežitel'no/ne-

${ }^{8} \mathrm{Zu}$ den Funktionen der Anführungszeichen als Prädikationsmodifikator oder Abweichungssignal cf. KLOCKOW 1980: 222-239 (dem wir den Terminus Applikationsvorbehalt entnehmen). Von slavistischer Seite hat sich MAYENOWA 1967 mit der Doppelbödigkeit von Wörtern, die in Anführungszeichen stehen, auseinandergesetzt; cf. bes. p. 1321 ihre Bemerkungen zur Analogie zwischen Anführungszeichen und Metapher, die sich ohne weiteres auf die morphologische Wortbildung übertragen lassen. 
prenebrežitel'no erreichen die suffigierten Wörter deutlich höhere Werte: košečka kann ironischer, verächtlicher und sogar mißbilligender als koška klingen (nach MJAGKova 1983: 104, cf. die Interpretation pp. 103-115 sowie MJaGKova 1986):

\begin{tabular}{||l|l|l|l|l|l|l||}
\hline \multirow{2}{*}{$\begin{array}{l}\text { Zur Beurteilung } \\
\text { vorgelegtes Wort }\end{array}$} & \multicolumn{7}{|c|}{ Anzahl der Bewertungen (in \%) } \\
\cline { 2 - 8 } & 1 & 2 & 3 & 4 & 5 & 6 \\
\hline koška & $75 / 13$ & $40 / 15$ & $30 / 33$ & $23 / 50$ & $48 / 10$ & $47 / 13$ \\
\hline košečka & $78 / 12$ & $48 / 22$ & $53 / 30$ & $32 / 52$ & $53 / 23$ & $62 / 18$ \\
\hline
\end{tabular}

Bedeutung der Spalten:
1: xorošo/ploxo
2: silno/slabo
3: ironično/neironično
4: prenebrežitel'o/neprenebrežitel'no
5: želatel'no/neželatel'no
6: odobritel'no/neodobritel'no

Aber auch die Verkleinerung gehört zur Wortbildungsbedeutung des Deminutivs nicht wesenhaft dazu, sondern ergibt sich indirekt daraus, daß eine starke Abweichung des Denotats von der Größe der übrigen Exemplare der Kategorie, in die es eingeordnet werden soll, diese Einordnung problematisch erscheinen läßt; diesen Vorbehalt bringt das Deminutiv zum Ausdruck. Gelegentlich wird das Deminutiv sogar zur Intensivierung gebraucht, wie in folgenden Beispielen, die unerklärlich erscheinen, wenn man von der Grundbedeutung ausgeht 'kleiner/schwächer... als das durchschnittliche Mitglied der Kategorie, die das Ausgangswort bezeichnet' (cf. Pijamovataja 1961: 20 sowie Mandel'sttam 1903, 8: 318).

Delo pod večer, zimoj

I morozec znatnyj.

(NeKRASOV, General Toptygin)

A lampy

kak stanut

nu, ja doložu vam --

noč’ kopat',

plamečko!

(MAJAKOVSKIJ, Brodvej) 
Nicht Emotionalität oder Verkleinerung, sondern spielerische Distanzierung vom eigenen Sprachgebrauch macht das Wesen des Deminutivs aus (cf. das Merkmal [non-serious] bei BARBARESI/DRESSLER 1994: 144). Dies erklärt den Gebrauch von Deminutiva als Höflichkeitsformen und rückt sie in die Nachbarschaft der Ironie und des Wortspiels. A. AXMATOVA hat dies in einem Gedicht über PUSKIN mit sicherem Instinkt zum Ausdruck gebracht:

Kto znaet, čto takoe slava!
Kakoj cenoj kupil on pravo,
Vozmožnost' ili blagodat'
Nad vsem tak mudro i lukavo
Sutit', tainstvenno molčat'
I nogu nožkoj nazyvat'?...

(A. Axmatova, Puškin)

Der Gebrauch des Deminutivs wird hier mit dem Scherz (dem spielerischen Gebrauch der Sprache) und dem Schweigen (dem Verzweifeln an ihrer Macht) in Verbindung gebracht.

Neben den expressiven fallen auch die taxinomischen Ableitungen in den Bereich der metaphorischen Wortbildung. Darunter verstehen wir Ableitungen wie bljudce 'Untertasse', jastrebok 'Jagdflugzeug' oder lodočki 'eine Art Damenschuhe'. Ihre metaphorische Grundstruktur ist ohne weiteres einsichtig: ein bljudce ist eben kein richtiges bljudo ('Teller, Schale'); das Jagdflugzeug, das als jastrebok bezeichnet wird, kein richtiger jastreb ('Habicht'); die lodočki, die man am Fuß trägt, keine richtigen lodki ('Boote'). Auf Grund ihrer metaphorischen Wortbildungsbedeutung gehen die Deminutiva am leichtesten in taxinomische Ableitungen über, doch umfaßt diese Kategorie Ableitungen mit sehr heterogener Herkunft; wir geben eine -- keineswegs erschöpfende -- Liste:

- Suffixale Ableitungen auf -oid: romboid, zebroid, negroid, planetoid, metalloid (das heute durch die Bezeichnung nemetall ersetzt ist), affiksoid (das neben poluaffiks gebraucht wird).

- Wörter, die mit psevdo-, kvazi-, polu- sowie dem heute nicht mehr produktiven pa-präfigiert sind: psevdogibrid; kvazizvezda; poluvagon, polukočevnik, polukustarnik; pasynok, padćerica (in den letzten zwei Beispielen liegt keine reine Präfigierung vor).

- Schließlich können auch gewisse Kernmorpheme ihre lexikalische Bedeutung einbüßen und zu Exponenten einer taxinomisch-metaphorischen Wortbildungsbedeutung werden, so im Russischen bab'e leto 'Altweibersommer'; $\mathrm{cf}$. im amerikanischen 
Englisch: Indian summer 'Altweibersommer', und im Neugriechischen: $\phi, \rho \alpha \curlyvee \kappa \circ \sigma \tau \dot{\alpha} \phi$ U $\lambda \circ$ 'Stachelbeere', $\phi \rho \alpha \curlyvee \kappa \delta \sigma$ UKo 'Feigendistel' (zusammengesetzt aus $\sigma \tau \alpha \phi u ́ \lambda_{l}$ 'Weintraube' bzw. oúko 'Feige' und $\phi \rho \alpha \dot{\gamma}$ KOS 'Westeuropäer, Katholik').

Wir führen noch einige Beispiele für die verschiedenen Spielarten der taxinomischen Wortbildung an:

1. Übertragung von der Art auf die Gattung (von einer Unter- zu einer Oberkategorie): $\check{z} u k \rightarrow \check{z} u c ̌ o k, ~ m u x a \rightarrow$ muška. ${ }^{10}$

2. Übertragung von der Gattung auf die Art (von einer Ober- zu einer Unterkategorie): gorox $\rightarrow$ gorošek, pirog $\rightarrow$ pirožok, svekla $\rightarrow$ sveklovica.

3. Übertragung zwischen zwei Arten derselben Gattung (zwischen Unterkategorien derselben Oberkategorie): bljudo $\rightarrow$ bljudce, čaša $\rightarrow$ čaška, gagara $\rightarrow$ gagarka.

4. Übertragung zwischen Arten verschiedener Gattungen (zwischen Unterkategorien verschiedener Oberkategorien): boroda $\rightarrow$ borodka, noga $\rightarrow$ nožka, golova $\rightarrow$ golovka.

In dem Wortbildungsmodell von M. DoKuLIL wird dem Unterschied in der Wortbildungsstruktur metonymischer und metaphorischer Ableitungen durch die Kategorien der Mutation (für Ableitungen wie vinogradar) und der Modifikation (für Ableitungen wie zimka) Rechnung getragen (DoKULIL 1962: 46-49; DoKULIL 1968: 209sq.; UluXANOV 1996: 149; cf. Kap. 2.2.2 dieser Arbeit). Am nächsten sind dem Begriff der metaphorischen Wortbildung DRESSLER und BARBARESI in ihrer Analyse der Deminutiva des Italienischen und des Deutschen gekommen (BARBARESI/DRESSLER 1994); doch lassen auch sie die sog. lexikalisierten Deminutiva, d.h. den Bereich der metaphorisch-taxinomischen Wortbildung, beiseite (cf. ibid. p. 135-137). Einen guten Überblick über das Bedeutungsspektrum des Deminutivs in verschiedenen Sprachen der Welt liefert die breit angelegte Studie von JURAFSKY 1996 (Vorgängerversion: JURAFSKY 1993); dem Zusammenspiel von Metapher und Metonymie bei der Entwicklung eines Substantivs mit der Bedeutung 'Kind' zum Deminutivsuf-

9 "Actually the term was one of several -- Indian summer, Indian corn and Indian tea, for example -- invented by the earliest colonists to describe something which resembled the genuine article as they had known it in England but actually was not the same" (MORRIS 1962: 190, s.v. Indian giver).

10 Cf. SARnowski 1991: 33, der von Generalisierung des Deminutivs spricht. Wir haben bereits bemerkt, daß diese Generalisierung in keinem Gegensatz zur Metaphorisierung steht, sondern nur eine ihrer Unterarten ist. 
fix gehen HEINE/CLAUDI/HÜNNEMEIER 1991: 79-97 am Beispiel der afrikanischen Sprache Ewe nach. In der Slavistik hat NORBERT REITER den Begriff der Perspektive für die Analyse von Deminutiva und Augmentativa fruchtbar zu machen versucht, ohne jedoch die Brücke zur Metapher zu schlagen (REITER 1988). Wo man vom Begriff der Ähnlichkeitsassoziation ausging, hat man nur den Teilbereich der taxinomisch-metaphorischen Wortbildung gesehen (MILOSLAVSKIJ 1980: 50-53) und sich dessen formale Nähe zur expressiv-metaphorischen Wortbildung nur schlecht erklären können (ZEMSKAJA 1992: 161sq., SARNOWSKI 1991: 13sq.). LOPATIN 1975 verwendet den Terminus metaforičeskaja motivacija, meint damit allerdings nicht metaphorisch-taxinomische (und noch viel weniger expressive) Ableitungen, sondern die figurative Bedeutung metonymischer Ableitungen wie neboskreb und lizobljud.

\subsection{Die Behandlung der Beziehungsadjektive}

Den Terminus Beziehungsadjektiv werden wir im folgenden unterschiedslos für alle desubstantivischen Adjektive gebrauchen. Diesen Adjektiven liegt nämlich stets die Vorstellung einer wie auch immer gearteten Beziehung zwischen dem Grundwort und dem durch das Adjektiv bestimmten Substantiv zugrunde (cf. WARREN 1988); nur durch seine vollständige Demotivierung hört ein solches Adjektiv auf, Beziehungsadjektiv zu sein. Die Unterscheidung von Beziehungs- und Qualitätsadjektiven innerhalb der Kategorie der desubstantivischen Adjektive, wie sie allgemein üblich ist (etwa lesistyj im Gegensatz zu lesnoj), bedient sich teils morphologischer (Bildung von Steigerungsformen, Bildung von nomina qualitatis), teils syntaktischer Kriterien (Fähigkeit, in prädikativer Stellung aufzutreten); ob sie vom Wortbildungsstandpunkt aus sinnvoll ist, d.h. ob es keine Wortbildungskategorien gibt, die sowohl Qualitäts- als auch Beziehungsadjektive i.e.S. enthalten, kann erst nach Abschluß der Analyse beurteilt werden.

Die besondere Stellung des Beziehungsadjektivs im Wortbildungsparadigma ergibt sich daraus, daß es erst in Verbindung mit einem bestimmten Substantiv eine vollständige Benennung ergibt. Von den oben erwähnten onomasiologischen Bestandteilen des abgeleiteten Wortes: der onomasiologischen Basis und dem onomasiologischen Merkmal, ist die Basis im Adjektiv ausgespart; lediglich seine Flexionsendung verweist auf die so entstandene Leerstelle, schließt sie jedoch nicht, wie etwa der Vergleich des Adjektivs gorbatyj mit dem Substantiv gorbun zeigt (cf. die treffenden Bemerkungen bei HEINZ 1956: 266sq.). Das Beziehungsadjektiv vertritt also in den mit seiner Hilfe gebildeten Mehrwortbenennungen lediglich das onomasiologische Merkmal, das man sich wie immer aus onomasiologischem Motiv und onomasiologischer Kopula zusammengesetzt denken kann; in suffixalen Ableitungen wird das onomasiologische Merkmal auf das onomasiologische Motiv reduziert (kartofel'nyj sup), in Komposita ist darüber hinaus die onomasiologische Kopula 
durch ein Kernmorphem oder einen abgeleiteten Wortstamm (kartofeleuboročnyj kombajn) vertreten.

Auch Verbindungen desubstantivischer Adjektive mit Substantiven lassen sich danach einteilen, ob die zugrundeliegende Beziehung metaphorisch oder metonymisch ist (HEINZ 1956, 1957). Metaphorisch sind z.B. bokal'čatyj stakan, štoporoobraznyj rog, kuvšinnyj sapog, ložečnoe sverlo, tareločnaja čečevica; metonymisch sind fljažnyj čexol, goršečnyj zavod, goršečnoe pivo, sitnaja muka, podnosnyj master, rjumočnaja nožka, pestovaja golovka, tareločnaja podstavka (die zitierten Fügungen stammen alle aus den benutzten Wörterbüchern und sind heute zum größten Teil veraltet; ihre Bedeutung läßt sich aus der Bedeutung der Bestandteile leicht erschließen).

In vielen Fügungen tritt die im vorangehenden Kapitel behandelte Dialektik metaphorischer und metonymischer Beziehungen zutage, z.B.
petušinyj golos 'Stimme, die an einen Hahnenschrei erinnert' orlinyj nos 'Adlernase' sokolinye oči 'Falkenaugen'

Solche Verbindungen weisen sowohl eine metaphorische als auch eine metonymische Komponente auf, die man sich auf zwei Stufen verteilt denken kann:

$$
\text { orel --[mtn]-- kljuv --[mph]-- nos }
$$

Eine bestimmte Schnabelform wird durch Verweis auf den Vogel, der sie aufweist, identifiziert, um anschließend als Vergleichsobjekt für die menschliche Nase zu dienen.

Eine solche Zerlegung läßt das Verhältnis zwischen der metaphorischen und der metonymischen Komponente in orlinyj nos selbst metonymisch erscheinen. Die Leichtigkeit, mit der solche Verbindungen gebildet und verstanden werden, und ihre formale Einfachheit (sie weisen schließlich nicht zwei, sondern ein Suffix auf) suggerieren eine metaphorische Interpretation des Verhältnisses der beiden Komponenten: wir hätten es demnach mit einer einfachen Beziehung zu tun, die bei der semantischen Analyse bald ihre metaphorische, bald ihre metonymische Seite hervorkehrt. Beide Interpretationen sind legitim."

Die Spezifik der Beziehungsadjektive bringt es mit sich, daß sich das Problem der Bestimmung ihrer Wortbildungsbedeutung auf zweifache Weise stellt:

" Zu solchen Fügungen cf. allgemein BEARD 1995: 220-227. 
a) Abgrenzung auf der paradigmatischen Achse. Hierbei ist zu entscheiden, ob zwei (oder mehrere) Beziehungsadjektive eines Wortbildungsparadigmas zu ein und derselben oder aber zu verschiedenen Wortbildungskategorien gehören. Das Prinzip der Relevanz verlangt, daß eine Zuordnung zu verschiedenen Wortbildungskategorien nicht einfach nach einer mehr oder weniger deutlich empfundenen Bedeutungsverschiedenheit, sondern nach Oppositionen erfolgt, die durch Substitution in identischen Kontexten ermittelt werden. Im Falle des Beziehungsadjektivs besteht dieser Kontext nicht nur aus der Ableitungsbasis, sondern auch aus den Substantiven, die mit den abgeleiteten Adjektiven verbunden werden können. So genügt etwa die Opposition pyl'nyj :: pylevoj 'staubig :: Staub-' für sich genommen noch nicht, um die Zugehörigkeit der beiden Adjektive zu verschiedenen Wortbildungskategorien zu beweisen. Es muß darüber hinaus (mindestens) ein Substantiv genannt werden können, das mit beiden Adjektiven verbunden wird und die Bedeutungsopposition zwischen ihnen illustriert: pyl'nye očki :: pylevye očki ('staubige Brille :: Staubschutzbrille'), cf. pyl'nyj/pylevoj ventiljator, pyl'nye/pylevye časticy (alle Beispiele nach ZEMSKAJA 1991: 154; zum Verhältnis zwischen den Suffixen -ov- und - $n$ - cf. ZEMSKAJA 1965). Nur auf diese Weise kann ein Paar wie pyl'nyj/pylevoj von jenen Fällen unterschieden werden, in denen sich zwei Beziehungsadjektive in ihrer lexikalischen Kompatibilität unterscheiden und unter deren Einfluß auch eigene Bedeutungsnuancen entwickeln, jedoch nie in ein und demselben Kontext zueinander in Opposition treten.

b) Abgrenzung auf der syntagmatischen Achse. Hierbei geht es um die Frage, welchen Anteil das Beziehungsadjektiv und welchen Anteil das davon bestimmte Substantiv am Zustandekommen der Gesamtbedeutung einer Mehrwortbenennung besitzen. Es ist offensichtlich, daß manche Beziehungsadjektive den Mehrwortbenennungen, in die sie eingehen, viel stärkere Beschränkungen auferlegen als andere: durch ein Adjektiv wie butylkomoečnyj 'Flaschenspül-' ist eine spezifische metonymisch-situative Beziehung vorgegeben, ein Adjektiv wie štoporoobraznyj 'spiralförmig' kann dagegen nur metaphorische Beziehungen ausdrücken, bei denen die Form des Gegenstandes, den die Ableitungsbasis bezeichnet, als tertium comparationis gilt. Dagegen ist durch višnevyj noch nicht einmal der elementare Charakter der Beziehung zwischen den beiden Substantiven bestimmt: diese kann ja metaphorisch wie in višnevyj barxat 'kirschroter Samt' oder metonymisch wie in višnevyj sad 'Kirschgarten' sein. Allgemein gilt: je unterschiedlicher die Beziehungen sind, die ein bestimmtes Adjektiv in Verbindung mit verschiedenen Substantiven ausdrücken kann, desto niedriger muß der Beitrag dieses Adjektivs zur Semantik der Gesamtverbindung veranschlagt werden (es sei denn, man entschließt sich dazu, mehrere homonyme Adjektivformen anzusetzen; zur Frage der Wortbildungshomonymie kehren wir in Kap. 3.5.2 zurück).

Verläßliche Aussagen über die Wortbildungsbedeutung adjektivischer Ableitungen könnten nur auf der Grundlage eines umfangreichen Korpus vorgenommen werden; 
das Material der Wörterbücher, die in diesem Bereich zudem besonders unzuverlässig sind (siehe ZEMSKAJA 1977), reicht dazu keineswegs aus. Wir haben deshalb im folgenden die meisten Dubletten, die in diesem Bereich auftreten (apel'sinovyj und apel'sinnyj, abrikosovyj und abrikosnyj etc.) zu ein und derselben Wortbildungskategorie zusammengefaßt, d.h. als synonym behandelt. Stichproben mit einzelnen Fügungen ergeben meist, daß der Austausch des einen Adjektivs gegen das andere von Muttersprachlern entweder abgelehnt oder als semantisch irrelevant angesehen wird; diese Frage bedarf allerdings weiterer Untersuchungen.

\subsection{Wortbildung und Polysemie}

In den folgenden zwei Abschnitten sollen jene Probleme behandelt werden, die für die Wortbildungsanalyse mit der Polysemie (bzw. Homonymie, Vagheit) des ableitenden oder des abgeleiteten Wortes verbunden sind.

\subsubsection{Die Mehrdeutigkeit der ableitenden Wörter}

Wir verwenden hier Mehrdeutigkeit als Oberbegriff für folgende semantische Beziehungen:

a) Homonymie: banka 'stekljannyj ili metalličeskij sosud cilindričeskoj formy' 'Einmachglas', 'v reči morjakov, rybakov -- doska dlja sidenija grebcov $v$ lodke' 'Ruderbank', 'vozvyšennyj učastok morskogo dna; podvodnaja otmel' 'Sandbank'.

b) Polysemie: fljaga 'ploskij sosud, inogda opletennyj ili obšityj čem-l., prisposoblennyj dlja nošenija s soboj' 'Feldflasche', 'bol'šoj sosud s ručkami dlja perevozki żidkostej; bidon' 'Kanister'; bljudo 'stolovaja posuda v vide bol'šoj tarelki dlja podači kušanjja' 'flache Schüssel, Schale', 'otdel'noe kušan'e iz čisla sostavljajuščix obed, zavtrak, ili užin' 'Gericht, Gang'. Im ersten Beispiel beruht die Polysemie auf einer metaphorischen, im zweiten auf einer metonymischen Beziehung.

c) Vagheit: diese kommt grundsätzlich jedem Wort im Sprachgebrauch zu. Die Kriterien, mit denen sie von der Polysemie abgegrenzt werden kann, werden bei CRuSE 1986: 54sqq. und ZwICKY/SADOCK 1975 diskutiert. Auf der Ebene des Sprachgebrauchs entspricht der Vagheit ein Phänomen, das wir mit CRUSE 1986: 53 als highlighting (bzw. backgrounding) semantischer Merkmale bezeichnen wollen: in razbit banku wird das Material, in varen'e $v$ bankax die Funktion von banka in den Vordergund der Aufmerksamkeit gerückt.

Es ist bekannt, daß die Abgrenzung dieser Erscheinungen voneinander sowohl in theoretischer als auch in praktischer Hinsicht große Schwierigkeiten bereitet. Für uns ist in erster Linie die Frage interessant: in welchen Fällen sollen wir diesen ver- 
schiedenen semantischen Einheiten auch verschiedene Wortbildungsparadigmen zuordnen, in welchen Fällen nicht? Bei den Homonymen wird uns die Entscheidung bereits durch die Struktur des SSS (und der ihm zugrundeliegenden Wörterbücher) abgenommen: jedes Wort erhält sein eigenes Wortbildungsparadigma. Dagegen kommt bei Fällen von Vagheit bzw. highlighting eine Aufspaltung der Wortbildungsparadigmen nicht in Betracht: da das ableitende Wort prinzipiell in jeder Ableitung unter einem verschiedenen Blickwinkel betrachtet wird, würde eine solche Lösung hier zu einer vollständigen Atomisierung der Wortbildungsparadigmen führen.

Vor ein echtes Dilemma stellen uns dagegen ableitende Wörter, die polysem sind; die Entscheidung zwischen der Bewahrung der Einheit des Wortbildungsparadigmas und seiner Aufspaltung unter Aufteilung der Ableitungen auf die einzelnen Bedeutungsvarianten des ableitenden Wortes ist hier nicht einfach, und wir werden zeigen, daß sie nur nach Plausibilitätskriterien getroffen werden kann. Dazu bemerken wir, daß sich die Polysemie des ableitenden Wortes im abgeleiteten Wort auf zweierlei Weise niederschlagen kann: sie kann entweder erhalten oder aufgelöst werden. Was damit gemeint ist, kann am besten durch einige Beispiele gezeigt werden.

Zu fljaga verzeichnen die Wörterbücher die beiden Bedeutungen 'Feldflasche' und 'Kanister'. In fljagomojka 'Kanisterspülmaschine', fljagomoečnyj 'Kanisterspül-' ist die Mehrdeutigkeit von fljaga aufgelöst, da die Basis in sie nur in einer ihrer Bedeutungsvarianten eingeht. Im Adjektiv fljažnyj ist diese Mehrdeutigkeit dagegen erhalten, da durch dieses Wort ein Bezug sowohl auf Feldflaschen als auch auf Kanister ausgedrückt werden kann, wobei der situative und sprachliche Kontext (in erster Linie das Substantiv, mit dem fljažnyj verbunden wird) die Entscheidung für das eine oder das andere ermöglicht: man vergleiche in dieser Hinsicht fljažnyj čexol, fljažnaja probka ('Futteral, Korken einer Feldflasche') mit fljažnoe moloko ('Kanistermilch'; alle Beispiele aus dem MAS). Ähnlich liegen die Dinge bei kotel, wofür MAS folgende Definitionen bietet: 'metalličeskij sosud okrugloj formy dlja varki pišči ili kipjačenija vody' und 'zakrytyj sosud dlja polučenija para iz vody'12. Die Mehrdeutigkeit ist in kotlopunkt 'stolovaja na lesoseke, polevom stane i td.' 'Feldküche' zugunsten der ersten, in kotloturbinnyj 'Kesselturbinen-' zugunsten der zweiten Bedeutungsvariante aufgelöst; in kotlovyj 'Pril. k kotel ( $v$ l i 2 znač.)' ist sie dagegen erhalten.

Am Wortbildungsparadigma von kotel läßt sich übrigens zeigen, daß die Zuordnung einer Ableitung zu einer der Bedeutungsvarianten des ableitenden Wortes auch dann Schwierigkeiten bereiten kann, wenn die Ableitung selbst nicht polysem ist; in den

${ }^{12}$ Eine dritte Bedeutungsvariante 'voen. polnoe okruženie (bol'šix grupp vojsk)' spielt in der Wortbildung keine Rolle und findet deshalb hier keine weitere Berücksichtigung. 
metaphorischen Ableitungen kotlovan 'Baugrube' und kotlovina 'Kesselschlucht' ist es müßig zu fragen, ob Ähnlichkeit mit einem Koch- oder einem Dampfkessel gemeint ist, da sich Koch- und Dampfkessel in erster Linie (wenn auch nicht ausschließlich) durch die Funktion unterscheiden, während das tertium comparationis der genannten Ableitungen in der Form liegt. Es wird bei einem Wort wie kotlovan auch nicht möglich sein, aufgrund des Satzkontextes zu entscheiden, ob nun die gegebene Bodensenkung größere Ähnlichkeit mit einem Dampf- oder einem Kochkessel hat ${ }^{13}$. Würde man sich zu einer Aufspaltung des Wortbildungsparadigmas von kotel entscheiden, so müßte man nicht nur Fälle wie kotlovyj, sondern auch kotlovan und kotlovina sowohl dem Wortbildungsparadigma der Bedeutungsvariante 'Dampfkessel' als auch dem Wortbildungsparadigma der Bedeutungsvariante 'Kochkessel' zuordnen.

Wir wollen nun zeigen, daß die Entscheidung über die Aufspaltung eines Wortbildungsparadigmas zugleich eine Entscheidung über die Abgrenzung des Gegenstandes der Wortbildung ist; sie kann deshalb im Rahmen der Wortbildungsanalyse selbst nicht getroffen werden. Wir wollen unsere These wieder an einem Beispiel entwickeln.

Wie die meisten Nutzpflanzen hat auch kartofel' eine doppelte Bedeutung, indem es sowohl die gesamte Pflanze als auch deren Teil, die Knolle, bezeichnet: '1. ogorodnoe rastenie sem. paslenovyx, ovošč. [...] 2. sobir. podzemnye klubni ètogo rastenija, upotrebljaemye v pišču'. Im Wortbildungsparadigma dieses Substantivs finden wir das Kompositum kartofelexranilišč 'Kartoffelmiete' und die Ableitung kartofljanišče '(abgeerntetes) Kartoffelfeld'. Bei deren Behandlung stehen zwei logisch gleichberechtigte Wege offen:

a) Man tastet die Einheit des Wortbildungsparadigmas von kartofel nicht an und setzt auf Grund der Opposition zwischen kartofelexranilišče und kartofljanišče zwei verschiedene Wortbildungskategorien an, deren Inhalt etwa mit 'Lagervorrichtung für...' und 'Feld, auf dem ... gewachsen ist' wiederzugeben wäre. Die beiden Bedeutungen des ableitenden Wortes erscheinen dann als kombinatorische Varianten, deren Wahl von dem Charakter des Formanten abhängt: das Endprodukt wird gelagert, die Pflanze angebaut.

b) Man stellt für jede der beiden Bedeutungen von kartofel' ein eigenes Wortbildungsparadigma auf. Nichts spricht dann dagegen, kartofelexranilišče und kartofljanišče als Realisationen ein und derselben Wortbildungskategorie anzusehen, deren nunmehr recht abstrakter Inhalt mit 'Ort, wo sich ... befindet' umschrieben

${ }^{13} \mathrm{Zu}$ kotloobraznyj gibt MAS allerdings die Definition 'imejuščij formu kotla (v l znac̆.)'. 
werden könnte. Jetzt sind die beiden Bedeutungen 'Lagervorrichtung' und 'Feld' (bzw. die onomasiologischen Kopulae 'lagern' und 'anbauen') kombinatorische Varianten der Wortbildungskategorie, die in Abhängigkeit vom lexikalischen Charakter der Ableitungsbasis gewählt werden: handelt es sich um die Pflanze, so ist der Ort, an dem sie sich naturgemäß befindet, das Feld; ist die Rede von ihrer bereits geernteten Knolle, so kann nur ein Lagerraum gemeint sein.

Auch hier geht es letzten Endes um nichts anderes als um die Grenze, die Wortbildung und Lexikologie trennt: die erste Lösung verschiebt diese Grenze zugunsten der Wortbildung (die Wortbildungsbedeutungen werden konkreter, lexikalischer), die zweite zugunsten der Lexikologie. Eine Entscheidung für die eine oder die andere Lösung setzt voraus, daß man diese Grenze nach einem systemexternen Gesichtspunkt bereits gezogen hat. Dies ist immer dann der Fall, wenn etwa behauptet wird, bestimmte Bedeutungen seien für die Definition der Wortbildungskategorien nicht abstrakt genug: es dürfe zwar nomina loci, aber keine nomina agri geben. Man muß sich jedoch darüber im klaren sein, daß man mit solchen apriorischen Behauptungen keine empirischen Aussagen über das Wortbildungssystem einer bestimmten Sprache trifft, sondern nur den Gebrauch des Terminus 'Wortbildungskategorie' auf mehr oder weniger sinnvolle, aber prinzipiell willkürliche Weise festlegt.

Eine zweite Möglichkeit, eine Entscheidung in unserem Fall herbeizuführen, bestünde darin, die Ausdrucksseite zur Richtschnur zu nehmen: die erste Lösung wäre demnach deshalb vorzuziehen, weil ja der semantische Unterschied zwischen kartofelexranilišče und kartofljanišče nicht am Wortstamm von kartofel', sondem an dessen Erweiterungen zum Ausdruck komme. Dieses Kriterium scheint auf den ersten Blick unanfechtbar, ist jedoch ebenso problematisch wie das erste, da es das sprachliche Zeichen nicht durch seinen Wert (valeur), sondern durch seine Ausdrucksseite (und damit wieder von einem systemexternen Standpunkt aus) zu definieren versucht. Es ist offensichtlich, daß ein und dieselbe Wortbildungskategorie durch mehrere Wortbildungsmittel, ja sogar durch mehrere Wortbildungsarten ausgedrückt werden kann, und daß umgekehrt hinter ein und derselben Ausdrucksseite des Wortstammes oft eine Vieizahl von Bedeutungen steht: nicht umsonst hat man vom asymmetrischen Dualismus des sprachlichen Zeichens gesprochen (SERGEJ KARCEVSKIJ; cf. die Einleitung dieser Arbeit).

Das Dilemma, vor dem wir stehen, wird übrigens bei DEBATY-LUCA 1986: 173sqq. auf eine unzulässige Weise vereinfacht. DEBATY-LUCA stellt einen Gegensatz auf zwischen den wahren Oppositionen, in denen die lexikalische Bedeutung der Basis identisch bleibt und nur die Wortbildungskategorie wechselt, und den Pseudooppositionen, denen unterschiedliche Bedeutungsvarianten der Basis zugrundeliegen. Als ein Beispiel der letzteren dienen ihm die Paare der deverbalen Substantive auf -ment und -age im Französischen: abattage/abattement, éclaircissage/éclaircissement etc. Im Gegensatz zwischen diesen beiden Suffixe hat man oft den Ausdruck einer 
aspektuellen Opposition sehen wollen. Im Verbalabstraktum auf -ment erscheine die Handlung als ein abgeschlossenes Ganzes: es sei also als perfektiv (punktuell, terminativ) anzusehen; -age stelle dagegen die Handlung in ihrem Verlaufe dar und müsse deshalb als imperfektiv (durativ, iterativ) bezeichnet werden (p. 177-188) ${ }^{14}$ DEBATYLUCA bemerkt dazu auf p. 179 seiner Théorie fonctionnelle de la suffixation:

Nous sommes en présence de "pseudo-oppositions"; les bases des dérivés ein -age et celles des dérivés ein -ment ne sont pas absolument identiques. Suivant les cas, l'aspect durativ, itérativ ou concret est dans abattre 'faire tomber, tuer', [...], éclaircir 'rendre moins sombre, rendre moins dense', [...]; laspect ponctuel, terminatif ou abstrait réside dans abattre 'ôter les forces', éclaircir 'rendre clair pour l'esprit' [...]. En somme, on a coutume de projeter sur -age et -ment -- au sens presque psychologique du terme -- des qualités qui leur sont absolument extérieures.

Wer entscheidet jedoch, daß Bedeutungselemente wie 'durativ' oder 'abstrakt' der Wortbildung absolut fremd sind und nur in die Semantik der Ableitungsbasen eingehen?

Als Ergebnis unserer Überlegungen halten wir fest, daß bei polysemen ableitenden Wörtern sowohl die Aufspaltung des Wortbildungsparadigmas als auch die Bewahrung seiner Einheit legitime Entscheidungen sind, die vor Beginn der Wortbildungsanalyse getroffen werden müssen. Wir haben uns bemüht, jede der fünf untersuchten Kategorien nach einheitlichen Gesichtspunkten zu behandeln, und werden unsere Entscheidungen im nächsten Kapitel von Fall zu Fall begründen.

\subsubsection{Die Mehrdeutigkeit der Ableitungen}

Bei der Aufstellung der Wortbildungsparadigmen haben wir Wörter, die in allen Flexionsformen miteinander absolut identisch sind, im SSS jedoch in getrennten Lemmata angeführt werden, zu einem einzigen Lexem zusammengefaßt. Wegen ihrer Zugehörigkeit zu ein und derselben Wortfamilie weisen solche Wörter stets gewisse Bedeutungsüberschneidungen auf und sind deshalb vom Standpunkt der allgemeinen Lexikologie aus nicht als homonym, sondern als polysem anzusehen. In der Wortbildungslehre spricht man meist von (Wortbildungs)homonymie; dieser Begriff hat allerdings bislang sehr unterschiedliche Interpretationen erfahren (TiXONOV 1971, SOBOleva 1980, Tixonov/Pardaev 1989: 46sqq., AXMANOVA 1974). Wir wollen

${ }^{14}$ Auf die Diskussion dieser Termini, die sich ja z.T. auf die Aktionsart und nicht auf den Aspekt beziehen, brauchen wir uns an dieser Stelle nicht einzulassen. 
überlegen, wie er im Rahmen der bislang gemachten Ausführungen sinnvoll definiert werden kann.

Das Prinzip der Ökonomie verbietet uns, semantische Oppositionen, die durch keine formale Opposition zum Ausdruck gebracht werden, zur Grundlage der Aufspaltung von Ableitungen und ihrer Zuordnung zu verschiedenen Wortbildungskategorien $\mathrm{zu}$ machen. Falls jedoch die betreffende semantische Opposition in anderen Wortbildungsparadigmen von einer formalen Opposition begleitet wird, so sind wir berechtigt, Wortbildungskategorien aufzustellen, mit denen auch polyseme (oder homonyme -- auf den Unterschied kommt es hier nicht an) Wörter ein und desselben Wortbildungsparadigmas voneinander abgegrenzt werden können. So ermöglicht uns etwa der formale Gegensatz zwischen dem Deminutivum gorošek und dem Singulativum gorošina auch klubnička, malinka oder smorodinka, die nach Aussage der Wörterbücher sowohl Deminutiva als auch Singulativa sind, in je zwei verschiedene Ableitungen aufzuspalten.

Wir werden Paare abgeleiteter Wörter, deren Mitglieder auf diese Weise voneinander abgegrenzt wurden, schlechthin als Wortbildungshomonyme bezeichnen. Prinzipiell ließe sich auch in diesem Bereich ein Unterschied zwischen Polysemie und Homonymie treffen -- je nachdem, wie groß der Anteil der gemeinsamen semantischen Komponenten der Wortbildungskategorien ist, denen die beiden Ableitungen angehören; diese Unterscheidung ist jedoch für die Konstitution der Wortbildungskategorien selbst ohne Belang und wird deshalb hier nicht weiterverfolgt.

Unser Vorgehen entspricht also auch in diesem Fall der Grundvoraussetzung der paradigmatischen Wortbildungsanalyse: der Annahme, daß allen Ableitungen einer bestimmten Gruppe von Wörtern dasselbe abstrakte Wortbildungsparadigma (dasselbe System von Wortbildungskategorien) zugrundeliegt. Damit wird auch die Parallelität zwischen Wortbildungs- und Flexionsparadigmen gewahrt: auch im Bereich der Deklination bleibt ja der Synkretismus zweier Kasus in einem einzelnen Paradigma so lange unberücksichtigt, wie der Unterschied zwischen ihnen in anderen Paradigmen derselben Sprachstufe belegt ist. (Bei dieser Gelegenheit wollen wir darauf hinweisen, daß der Eindruck der Regelmäßigkeit, den die Flexionsparadigmen im Gegensatz zu den Wortbildungsparadigmen hervorrufen, zu einem nicht geringen Teil darauf zurückzuführen sein dürfte, daß im Bereich der Flexion die Synkretismen sowie die Defektivität vieler Paradigmen zugunsten eines weitgehend fiktiven Normalsystems ausgeklammert werden ${ }^{15}$.) Es darf allerdings nicht verschwiegen werden, daß die Aufspaltung der Wortbildungshomonyme nach unserem Kriterium von einem lexikologischen Standpunkt aus nicht ganz gerechtfertigt ist: sie entspricht den Polysemiekriterien, die bei ZWICKY/SADOCK 1975: 4-7 unter dem Titel appeals to semantic differentiae behandelt werden und als äußerst unzuverlässig gelten. Aus der

is Zum Begriff des Normalsystems s. HJELMSLEV 1935/37: 1,81sqq. 
Tatsache, daß eine semantische Opposition an bestimmten Stellen des lexikalischen Systems einer Sprache formal markiert ist, kann man nicht ohne weiteres schließen, daß wir es in den Fällen, in denen sie unausgedrückt bleibt, mit Polysemie und nicht mit Vagheit zu tun haben. Um dies an einem Beispiel zu verdeutlichen: im Bereich der Gewässerbezeichnungen des Russischen wird die semantische Opposition 'künstlich :: natürlich' durch das Paar kanal :: reka repräsentiert. Es wäre unzulässig, daraus den Schluß zu ziehen, vodopad sei ein polysemes Lexem mit den beiden Bedeutungen '1. künstlicher, 2. natürlicher Wasserfall'. Vielmehr ist vodopad im Hinblick auf diese semantische Opposition einfach vage (nichtspezifisch).

Es ist deshalb zu betonen, daß wir die nach dem hier aufgestellten Verfahren getrennten Wortbildungshomonyme nicht ohne weiteres als Fälle von lexikalischer Polysemie ansehen dürfen. Für die Wortbildung sind sie durch ihre Zugehörigkeit zu unterschiedlichen Wortbildungskategorien eindeutig als zwei verschiedene Einheiten gekennzeichnet; ob sie auch im lexikalischen System des Russischen als solche fungieren, ob also die Lexik die Möglichkeiten, die ihr die Wortbildung zur Verfügung stellt, an diesen Stellen voll ausnutzt, müßte getrennt untersucht werden. 


\section{PARADIGMATISCHE WORTBILDUNGSANALYSE: ANWENDUNGEN}

In diesem Kapitel wollen wir die Methode der paradigmatischen Wortbildungsanalyse auf fünf Gruppen denominaler Wortbildungsparadigmen anwenden. Wie wir bereits in Kapitel 1 erläutert haben, entsprechen die fünf Gruppen folgenden natürlichen Kategorien: frukty (Obst), ovošči (Gemüse), pticy (Vögel), posuda (Küchengeschirr und Besteck), sredstva peredviženija (Fortbewegungsmittel). Wir werden jeder der vier ersten Gruppen von Wortbildungsparadigmen ihr abstraktes Wortbildungsparadigma zuordnen, d.h. ein bestimmtes Inventar von Wortbildungskategorien; zu jeder Wortbildungskategorie wird eine umfassende, jedoch in der Regel nicht erschöpfende Liste von Ableitungen geliefert, in denen die entsprechende Kategorie realisiert ist.

\subsection{Analyse der Wortbildungsparadigmen der Kategorie Frukty (Obst)}

\subsubsection{Zum Bestand der Kategorie}

Als Grundlage unserer Untersuchung wählen wir die Ergebnisse des Produktionsexperiments, das wir im September/Oktober 1993 in Moskau durchgeführt haben; die Antworten auf den Stimulus frukty umfassen 35 verschiedene Lexeme (s. Anhang 1), darunter auch den Stimulus selbst (frukty) sowie die Bezeichnung einer beigeordneten Kategorie (ovošči).

Die Liste, die R. M. FrUMKINA und ihre Mitarbeiter ihren Untersuchungen zugrundegelegt haben (FRUMKINA/MIXEEV/MOSTOVAJA/RJUMINA 1991: 65), wollen wir nicht heranziehen: die genannten Autoren haben nämlich nicht nur Obstbezeichnungen, sondern Bezeichnungen für Mitglieder der übergeordneten Kategorie ("tematičeskaja gruppa") plody $i$ ovošči untersucht. Dabei hat sich herausgestellt, daß die genannte Kategorie in vier Teilkategorien zerfällt, die man als "jagody", "frukty", "ovošči" und "zelen" bezeichnen kann (ibid., pp. 96sqq.). Diese Vierteilung kann zwar leicht auf die Dichotomie von "frukty" vs. "ovošči" zurückgeführt werden: viele Bezeichnungen, die nach R. M. Frumkina et al. in die Teilkategorie "jagody" bzw. "zelen"" einzuordnen sind, wurden von unseren Versuchspersonen als Mitglieder der Kategorie "frukty" bzw. "ovošči" genannt. Trotzdem sind die Diskrepanzen zwischen unserer Liste und der Liste, die man durch die Vereinigung der Teilkategorien "jagody" und "frukty" bei FrUMKINA/MIXEEV/MOSTOVAJA/RJUMINA 1991: 97sq. gewinnen kann, zu groß, als daß man die beiden Listen zu einer einzigen verschmelzen könnte.

Die Versuchspersonen von V. N. MASKADYNJA haben mehrere Wörter genannt, die mit der Kategorie frukty durch eine Kontiguitätsassoziation verbunden sind: urožaj, vitaminy, jablonja; dazu gehören auch einige Wörter, die nicht Obstarten, sondern die aus ihnen gewonnenen Lebensmittel bezeichnen: urjuk, varen'e, kompot, sok. Auch diese Wörter wollen wir nicht in den Geltungsbereich unseres abstrakten Wortbildungsparadigmas einschließen. 


\subsubsection{Zum Bestand der Wortbildungsparadigmen}

Die überwältigende Mehrheit der Substantive in der Kategorie frukty (Obst) besitzt zwei Bedeutungen, indem durch sie nicht nur die Frucht, sondern auch die Pflanze bezeichnet wird. Die Mehrdeutigkeit der Basissubstantive kann, wie bereits besprochen, in den Ableitungen auf zweierlei Weise widergespiegelt werden:

a) die Mehrdeutigkeit der Basis bleibt in der Ableitung erhalten. Beispiele: gruška, das von BAS2 zu gruša sowohl in der Bedeutung 'Birne' als auch in der Bedeutung 'Birnbaum' gestellt wird; orexovyj in orexovaja skorlupa und orexovaja rošča.

b) die Mehrdeutigkeit der Basis wird in der Ableitung aufgelöst. Beispiele: gruševidnyj 'imejuščij vid, formu gruši (vo 2 znač.)'; vinogradnik 'učastok, zasažennyj vinogradom'.

Als erstes ist demnach zu fragen, ob eine bestimmte Ableitung die Mehrdeutigkeit der Basis reproduziert -- sei es direkt (wie in gruška) oder indirekt (wie in grušovnik '1. Garten, 2. Getränk'). Falls die Ableitung nicht mehrdeutig ist, ist zu entscheiden, welcher Bedeutungsvariante des Grundwortes man sie zuordnen will. Diese letzte Entscheidung kann allerdings nicht immer mit hinreichender Plausibilität getroffen werden. Der Situationszusammenhang zwischen Frucht und Pflanze ist nämlich so eng, daß eine Ableitung, die sich primär auf die Pflanze bezieht, in der Regel auch einen indirekten Bezug zur Frucht ausdrückt: so etwa eževičnik 'Brombeerdickicht', klubničnik 'Erdbeerbeete', mandarinnik 'Mandarinengarten, Mandarinenplantage'. Aus diesem Grund verzichten wir darauf, die Wortbildungsparadigmen der Substantive der Kategorie frukty (Obst) aufzuspalten.

\subsubsection{Das abstrakte Wortbildungsparadigma der Kategorie Frukty (Obst)}

Die substantivische Zone des Wortbildungsparadigmas umfaßt elf Wortbildungskategorien. Die expressive Wortbildung ist fast ausschließlich durch Deminutiva der ersten Ableitungsstufe vertreten:

' Zugunsten der Zuordnung von eževičnik, mandarinnik etc. zur Bedeutungsvariante 'Pflanze' spricht die Tatsache, daß in der Wortfamilie von jabloko die entsprechende Ableitung jablonja formal näher steht als jabloko: jablonnik 'Apfelgarten'. Absolute Beweiskraft kommt dem formalen Kriterium freilich nicht zu: jablonovka 'Apfelwein, Cider' steht formal jablonja näher als jabloko (cf. die Varianten jablonevyj/jablonovyj); auf der Inhaltsseite ist es freilich genau umgekehrt. 
a) Deminutiva (DEM): abrikosik, ananasik, apel'sinčik, arbuzik, čerešenka, dyn’ka, gruška, jabločko, klubnička, limončik, malinka, mandarinčik, persiček, rjabinka, rjabinuška, slivka, smorodinka, tykovka, višenka, zemljanička.

Die Wörterbücher verzeichnen ein einziges Augmentativum:

b) Augmentativa (AUG): arbuzišče.

Stark vertreten sind in den Wortbildungsparadigmen taxinomische Ableitungen -Bezeichnungen für Mitglieder derselben (grušovka) oder benachbarter Kategorien (Tiere: limonnica, seltener Artefakte: limonka). Die verbindende Assoziation ist in der Regel metaphorischer, gelegentlich aber auch metonymischer Natur (cf. Kap. 3.3).

c) Taxinomische Ableitungen (TAX): bananoed(y) 'Musophagidae', grušovka 'eine Art süßer, mehliger Äpfel, Birnapfel', jabločko 'Mittelpunkt der Zielscheibe' 'eine Art Matrosentanz', limonka 'eine Art Birnen, deren Form an eine Zitrone erinnert' 'prost. zitronenförmige Handgranate', limonnik 'bot. Schizandra', limonnica 'zool. Gonepteryx rhamni, ein Schmetterling der Familie der Kohlweißlinge mit zitronengelben Flügeln beim Männchen', malinovka 'Rotkehlchen', rjabinnik 'Misteldrossel, Krammetsvogel', ternosliv 'Schlehenpflaume' (zu tern und sliva), ternie 'jedes Stachelgewächs, Dorngesträuch', vinogradovnik 'Ampelopsis', višennik 'Cerasus fruticosa, Steppenkirsche', višnjak dass., višnesliva 'Schlehe, Schlehenpflaume'.

Die Fachsprache der Biologie besitzt einen eigenen Wortbildungstyp zur Bezeichnung übergeordneter Taxa:

d) Biologische Taxonyme (GEN): ananasnye 'Bromeliaceae', granatovye 'Punikazeen (Granatapfelgewächse)', jablonevye 'Maloideae, Pomoideae, Pyroideae' (zu jablonja), kryžovnikovye 'Grossulariaceae', slivovye 'Prunoideae, Amygdaloideae', tykvennye 'Cucurbitaceae, Kürbisgewächse', vinogradnye 'Vitaceae', vinogradovye 'Vitaceae'.

Im Bereich der metonymischen Wortbildung lassen sich Personen- und Gegenstandsbezeichnungen unterscheiden. Den Personenbezeichnungen liegen unterschiedliche Prädikate zugrunde: 'verkaufen', 'mögen', 'anbauen'. Kein Wortbildungsparadigma enthält jedoch zwei nichtsynonyme, formal verschiedene Ableitungen in diesem Bereich, so daß sich eine Differenzierung erübrigt.

e) Männliche bzw. neutrale Personenbezeichnungen (HOM): grušnik 'Verkäufer von gedämpften oder getrockneten Birnen', jabločnik 'Apfelverkäufer', malinnik 'Liebhaber von Himbeeren', vinogradar' 'Winzer, Weinbauer'. 
f) Weibliche Personenbezeichnungen (FEM): jabločnica 'Apfelverkäuferin', malinnica 'Liebhaberin von Himbeeren'.

Bei Paaren wie malinnik/malinnica, jabločnik/jabločnica sind strenggenommen zwei verschiedene Fälle denkbar:

-- Die zugrundeliegende Opposition ist äquipollent.

-- Die zugrundeliegende Opposition ist privativ. Die weiblichen Personenbezeichnungen stellen das markierte Glied der Opposition dar, die männlichen Personenbezeichnungen das unmarkierte, das bei Aufhebung der Opposition benutzt wird.

Wir vermuten, daß die Grenze zwischen diesen beiden Fällen weit weniger scharf ist, als es etwa bei ZEMSKAJA 1973: 249sq. dargestellt wird; außerdem ist in diesem Bereich der russischen Literatursprache der Gegenwart eine ausgeprägte Übergangstendenz äquipollenter Oppositionen in privative zu verzeichnen (cf. PANOV 1968, III: 191-213). In bestimmten Positionen (etwa in Verbindung mit dem Pluralmorphem) ist eine Neutralisierung auch bei Paaren wie moskvič/moskvicka oder jabločnik/jabločnica nicht auszuschließen, wobei stets die männliche Personenbezeichnung beide Oppositionsglieder vertritt. Das genaue Verhalten unserer Ableitungen in solchen Positionen müßte Gegenstand einer eigenen Untersuchung werden. Für unsere Zwecke genügt es anzumerken, daß der semantische Unterschied zwischen allgemeinen, geschlechtsneutralen und spezifisch männlichen Personenbezeichnungen in keinem Wortbildungsparadigma durch eine formale Opposition zum Ausdruck gebracht wird; es ist deshalb überflüssig, die Wortbildungskategorie HOM nach diesem Kriterium in Unterkategorien aufzuspalten.

Was die unbelebten Substantive in unseren Wortbildungsparadigmen betrifft, so verteilen sie sich auf mehrere Wortbildungskategorien. Zwei von ihnen haben ein deutliches semantisches Profil: Ableitungen mit lokaler Komponente (limonarij, smorodinnik etc.) und Ableitungen auf -ovka, mit denen (in der Regel hausgemachte) Branntweine bezeichnet werden: klubnikovka, rjabinovka etc.

g) Gegenstandsbezeichnungen mit lokaler Komponente (LOC): banannik 'Bananenbaum', eževičnik 'Brombeerdickicht', granatnik 'Granatbaum', gruševina 'Bimbaum', grušina 'Birnbaum', grušica 'Birnbaum', grušovnik 'Bimgarten', jablonnik 'A pfelgarten' (zu jablonja), jablonja 'Apfelbaum' (zu jabloko), klubničnik 'Erdbeerkraut, Erdbeerbeete', limonarij 'Pflanzstätte für Zitronen', malinnik 'Himbeergesträuch, -gebüsch', mandarinnik 'Mandarinengarten, Mandarinenplantage', mangifera 'Mangobaum, Mangifera', rjabinnik 'Ebereschenwäldchen, -gehölz', slivnjak 'Pflaumengarten', smorodinnik 'Johannisbeergesträuch, -hecke', ternovnik 'Schlehdom, Schlehe', tykvennik 'Kürbisgewächse' (nach dem Beleg in BAS1 auch 'Kürbisbeet' möglich; die 
Erklärung im BASI: 'Sobir. tykvennye rastenija'), vinogradnik 'Weinberg', višennik 'Kirschgarten', višnjak dass.

Die Beispiele zeigen, daß die lokale Grundbedeutung dieser Ableitungen in zwei Ausprägungen vorliegt: manche Ableitungen bezeichnen die Pflanze, an der die Frucht wächst: banannik, mangifera; andere wiederum bezeichnen ein Stück Boden, das mit der betreffenden Pflanze bewachsen ist: vinogradnik.

Was die Relevanz der semantischen Opposition zwischen 'Baum' und 'Boden' betrifft, so verhalten sich die Mitglieder unserer Kategorie nicht einheitlich. Bei Früchten, die an Bäumen wachsen, ist die Unterscheidung zwischen dem Baum selbst und dem Boden, auf dem er wächst, ohne weiteres sinnvoll. Im Wortbildungsparadigma von gruša finden wir dafür das Oppositionspaar gruševina vs. grušovnik (mit der veralteten, nur in BAS1 und PAW verzeichneten Bedeutung 'Birngarten'); cf. jablonja vs. jablonnik, die freilich nach unseren Regeln keine richtige Opposition bilden, weil sie auf verschiedenen Ableitungsstufen stehen. Anders liegen die Dinge bei Beeren, die in dichten Kolonien wachsen: hier ist eine Differenzierung zwischen den Pflanzen einer solchen Kolonie und dem Waldstück, das sie bedecken, nicht notwendig. Zwar wird in den Wörterbucherklärungen dem Gebüsch, das die betreffenden Pflanzen bilden (mit Ausdrücken wie 'kusty, kustiki, kustarnik, porosl', zarosli'), der Ort ('mesto'), an dem sie wachsen, gegenübergestellt:

brusničnik '1. Zarosli brusniki. 2. Mesto, porosšee brusnikoj' (MAS), višennik 'sad iz višnevyx derev'ev; zarosli višni' (BAS2), eževičnik 'zarosli eževiki' (MAS), 'mesto, sploš porosšee eževikoj' (UŠA), klubničnik '1. Mesto, zasažennoe klubnikoj. 2. Kust klubniki' (USA), smorodinnik 'kusty smorodiny, rastuščie vmeste, rjadom, $v$ bol'šom količestve; mesto, zanjatoe ètimi kustami' (UŠA). Cf. jagodnik '1. Mesto, gde rastut, vyraščivajutsja jagodnye rastenija. 2. Jagodnyj kust, jagodnoe rastenie' (MAS). -- Gelegentlich wird sogar eine noch differenziertere Aufgliederung der Bedeutung dieser Wörter vorgenommen, indem zusätzlich zwischen 'kust' und 'zarosli' unterschieden wird (so bei kizil'nik im USA).

Es ist jedoch sehr fraglich, ob sich hinter diesen Unterscheidungen echte Polysemie verbirgt oder ob es sich nicht vielmehr um Vagheit (oder sogar bloßes highlighting) handelt. Für letztere Annahme spricht die Inkonsequenz, die die Wörterbücher bei der Anführung der einen oder der anderen Bedeutungsvariante an den Tag legen, indem sie nicht nur einander widersprechen (so in dem angeführten Beispiel eževičnik; cf. černičnik), sondern auch sich selber. Jedenfalls wird -- anders als bei echten Fruchtbezeichungen wie gruša und jabloko -- die semantische Opposition zwischen 'Pflan$z^{\prime}$ ' und 'Boden' in keinem einzigen dieser Wortbildungsparadigmen durch eine formale Opposition zum Ausdruck gebracht. 
Diese Verhältnisse liefern ein Argument dafür, die Mitglieder der Kategorie frukty (im engeren, von FrUMKINA/MIXEEV/MOSTOVAJA/RJUMINA 1991: 96sqq. analysierten Sinn) und die Mitglieder der Kategorie jagody nicht in den Geltungsbereich desselben abstrakten Wortbildungsparadigmas einzuschließen. Es ist nicht möglich, im Rahmen eines einzigen abstrakten Wortbildungsparadigmas der Eigenart beider Kategorien gerecht zu werden: eine Aufspaltung der Wortbildungskategorie LOC in die Teilkategorien 'Feld' und 'Baum', wie sie durch die Opposition zwischen gruševina und grušovnik nahegelegt wird, findet in den Wortbildungsparadigmen der Beerenbezeichnungen keine Rechtfertigung.

h) Bezeichnungen für Fruchtbranntweine (LIQ): abrikosovka '(einheimischer) Aprikosenliqueur', grušovka 'Birnwein, Birnbranntwein', jablonovka 'schäumendes Getränk, das aus dem Saft von gekochten Äpfeln hergestellt wird; Apfelwein, Cider', klubnikovka 'Erdbeerbranntwein', limonovka 'Zitronenbranntwein', malinovka 'Himbeerbranntwein', rjabinovka 'Ebereschenwein', slivjanka, slivnjak, slivovica 'Pflaumenliqueur', ternovka 'Fruchtliqueur aus Schlehdornfrüchten oder Schlehenpflaumen', višnevka 'Kirschliqueur'.

Von einem genetischen Standpunkt aus ist anzumerken, daß die Ableitungen auf - ovka keine rein deadjektivischen Bildungen sind, sondern Univerbierungen der Mehrwortbenennungen abrikosovaja nastojka (oder nalivka), malinovaja nastojka (oder nalivka) etc. (cf. ISAĆENKO 1976: 176):

$$
\begin{aligned}
& \text { abrikos } \rightarrow \text { abrikosovaja nastojka } \rightarrow \text { abrikosovka } \\
& \text { malina } \rightarrow \text { malinovaja nastojka } \rightarrow \text { malinovka }
\end{aligned}
$$

Da das Zwischenglied hier substantivischen Charakter trägt, wären abrikosovka, malinovka etc. aus dem Wortbildungsparadigma von abrikos, malina etc. zu streichen. Um freilich abrikosovka von abrikosovaja nastojka abzuleiten, müßten wir: a) Mehrwortbenennungen in unser Korpus aufnehmen (s. dazu Kapitel 2.1) und b) die Ableitungsbeziehung in geeigneter Weise umdefinieren, so daß sie nicht nur Einzelwörter, sondern auch Syntagmen umfaßt. Es ist fraglich, ob eine solche Erweiterung des Wortbildungsbegriffs in diesem Fall wirklich sinnvoll ist: den Sprechern steht jederzeit die Möglichkeit offen, nach der Proportion abrikos ::abrikosovka, malina :: malinovka etc. neue Ableitungen zu bilden, ohne den Umweg über die Mehrwortbenennung einzuschlagen; -ovka wird in diesem Fall nicht als Verbindung von -ov- und $-k(a)$, sondern als ein einziges Suffix behandelt. Tatsächlich enthält unser Korpus eine Ableitung auf -ovka, die kein Beziehungsadjektiv auf -ovyj neben sich hat (klubnikovka) sowie eine andere, bei der sich die Umschreibung mit dem Beziehungsadjektiv aus semantischen Gründen verbietet (jablonovka).

Diese Herkunft aus Univerbierungen von Fügungen mit den Substantiven nastojka oder nalivka hat zur Folge, daß die Wortbildungsbedeutung der Kategorie LIQ sehr 
spezifisch ist. Eher allgemein ist dagegen die Wortbildungsbedeutung in den folgenden Ableitungen, die nichts weiter als das Vorhandensein einer metonymischen Beziehung zwischen zwei Gegenständen ausdrücken. Die Beziehungen selbst können unterschiedlichster Art sein: wir finden hier Bezeichnungen für chemische Substanzen (limonen); für Gegenstände, mit denen die Frucht bearbeitet (jablokorezka) oder transportiert werden kann (bananovoz); für Nahrungsmittel, die aus der Frucht hergestellt werden (abrikotin, limonad); und für Teile der Pflanze, die -- neben der Frucht selbst -- genutzt werden können (višnjak):

i) Allgemeine Gegenstandsbezeichnungen (RES): abrikotin 'Aprikosenliqueur' (die heute vorherrschende Bedeutung 'eine Art Torte mit farbiger Glasur' ist in den benutzten Wörterbüchern nicht verzeichnet), bananovoz 'Bananenfrachter', grušovnik 'Birnwein, eine Art Kwas aus Birnen', jablokorezka 'Apfelschneidemaschine', limonad 'Limonade', limonen 'das Limonen, 1-Methyl-4-isopropylzyklohexadien- $(1,8)$ ', černosliv 'getrocknete Pflaumen von großen und dunklen Pflaumensorten', tykvennik 'Kürbisbrei, Kürbisgrütze', vinogradarstvo 'Weinanbau, Wissenschaft vom Weinanbau', višennik 'Kirschholz', višnjak 'Kirschbaumruten'.

In unseren Wortbildungsparadigmen kommen schließlich zahlreiche Singulativa und ein einziges Kollektivum vor:

j) Singulativa (SIN): malinina 'Sing. zu malina', ternina 'Schlehdornfrucht', tykvina 'Kürbiskopf', vinogradina '(einzelne) Weinbeere', vinogradinka '(einzelne) Weinbeere' (so BAS-2, UŠA und BAS-1 noch als Deminutiv zum vorhergehenden; zur Entwicklung von -inka zu einem reinen Deminutivsuffix cf. ZEMSKAJA 1975b), zemljaničina 'Singulativum zu zemljanika'. -- Singulative Nebenbedeutungen haben auch zahlreiche Deminutiva wie z.B. zemljanička, die demnach zwei verschiedenen Wortbildungskategorien zuzuordnen sind (cf. hierzu 3.5.2).

Die Kategorie SIN bildet in gewissem Sinn das Gegenstück zu LOC (die in Ableitungen wie malinnik, eževičnik eine kollektive Nebenbedeutung aufweist): beide bilden Ableitungen, in denen die Polysemie der Ableitungsbasen aufgelöst wird; mit LOC werden Pflanzen-, mit SIN Fruchtbezeichnungen gebildet. Auch in SIN ist es weniger die abstrakte Kategorie der Quantität als die metonymische Beziehung zwischen dem Ganzen (der Pflanze) und dem Teil (der Frucht), die in den Ableitungen zum Ausdruck kommt.

k) Kollektivum (COL): višen’e.

Auch die adjektivische Zone ist in unseren Wortbildungsparadigmen durch zahlreiche Ableitungen vertreten. In Verbindung mit Substantiven drücken Adjektive, die von 
Mitgliedern der Kategorie frukty (Obst) abgeleitet sind, sehr unterschiedliche Beziehungen aus, von denen einige mit einer gewissen Regelmäßigkeit wiederkehren:

a) Hergestellt aus

- der Frucht: ajvovyj sok, vinogradovyj uksus, malinovaja nalivka.

- dem Holz: gruševaja doska, rjabinovoe udilišče.

b) Teil

- der Frucht: alyčovaja kostočka, apel'sinovye korki, vinogradovaja kist', limonnaja korka, tykvennoe semečko.

- des Baumes bzw. der Pflanze: alyčovaja vetka, ananasnye listja. ananasnye plody.

c) Bewachsen mit: ananasnaja plantacija, apel'sinovaja rošča, gruševyj sad, klubničnaja grjada.

d) Eigenschaft von: zemljaničnyj zapax, ananasnyj aromat, limonnyj cvet.

Selbstverständlich könnte man den hier aufgezählten Beziehungen weitere hinzufügen (nicht jede Fügung, die in den Wörterbüchern verzeichnet ist, läßt sich in ihnen zwanglos unterbringen), sie stärker differenzieren oder, umgekehrt, zu noch allgemeineren Beziehungstypen zusammenfassen. Im Rahmen einer paradigmatisch orientierten Wortbildungsanalyse sind jedoch nur jene inhaltlichen Einteilungen von Belang, die durch Oppositionen auf der Ausdrucksebene gerechtfertigt werden können. Wie verhalten sich unsere Wortbildungsparadigmen in dieser Hinsicht?

Eine Reihe von abgeleiteten Adjektiven kann sowohl metaphorische als auch metonymische Beziehungen zum Ausdruck bringen:

višnevyj sad vs. višnevyj barxat apel'sinovaja rošča vs. apel'sinovaja poloska zari

brusnicnoe varenie vs. brusnicnyj zakat

limonnaja vodka vs. limonnaja perčatka malinovaja alleja vs. malinovaja podkladka smorodinnyj kust vs. smorodinnye glaza

Schließlich gibt es Fügungen, in denen sowohl eine metaphorische als auch eine metonymische Komponente vorliegt: zemljaničnyj zapax, ananasnyj aromat, limonnyj cvet. $^{2}$

${ }^{2}$ Cf. HeInZ 1956: 24sq., BEARD 1993: 720 (wo übrigens diese "Entdeckung" SUSSEX 1974 zugeschrieben wird), BEARD 1995: 220-225. 
Wie in 3.4 dargelegt, steht die Breite der Beziehungen, die ein abgeleitetes Adjektiv eingehen kann, in umgekehrtem Verhältnis zu seinem semantischen Gehalt; entsprechend abstrakt muß die Wortbildungskategorie sein, der Adjektive wie malinovyj oder višnevyj angehören. Eine weitere Differenzierung ist unter ihnen nicht nötig.

Nun gibt es freilich eine Reihe von Wortbildungsparadigmen, in denen mehrere Beziehungsadjektive ohne klare semantische Abgrenzung miteinander konkurrieren. In den meisten Fällen handelt es sich um das Nebeneinander der Suffixe -ov(yj) und -n(yj): abrikosnyj und abrikosovyj, limonnyj und limonovyj etc. In kaum einem Falle decken sich die Musterfügungen, die die Wörterbücher zu dem einen dieser Adjektive liefern, mit denen seines Partners im Wortbildungsparadigma; daß die Wortwahl in diesem Bereich alles andere als frei ist, zeigen auch die Reaktionen der Muttersprachler, die Wendungen wie apel'sinnaja poloska zari oder limonovaja perčatka zugunsten von apel'sinovaja poloska zari bzw. limonnaja perčatka verwerfen (wobei sie in der Regel anmerken, daß diese Art von Fügung überhaupt ungewöhnlich ist). Es ist naheliegend, diese ungleiche Verteilung als reine Zufälligkeit des Sprachgebrauchs anzusehen (cf. ZEMSKAJA 1967: 95sq. und PAVLOV 1960: 68sq.), also nicht der langue, sondern der parole (oder allenfalls der Norm im Sinne COSERIUs) zuzurechnen. Diese Erklärung muß nur dort aufgegeben werden, wo der Austausch des einen Adjektivs durch das andere vor ein und demselben Substantiv zu einer Umdeutung der ausgedrückten Beziehung führt. Da jede Sprache die Tendenz hat, formale Varianten funktional auszunutzen, so daß die Ungleichheiten, die sich herausbilden, "zunehmend zur Polarisierung führen und [...] irgendwann in eine semantische Differenzierung einmünden" (REITER 1986: 145), wird es auch unter den Adjektiven auf $-n(y j)$ und $-o v(y j)$ einige Fälle dieser Art geben. Trotzdem gestattet unser beschränktes Ausgangsmaterial keine verläßliche Differenzierung dieser Beziehungsadjektive; es scheint sogar, daß diese Differenzierung im untersuchten Wortschatzbereich im allgemeinen ausbleibt (cf. ZEMSKAJA 1991: 153). Wir werden deshalb vorsichtshalber annehmen, daß sie alle prinzipiell in der Lage sind, sowohl metaphorische als auch metonymische Beziehungen auszudrücken (cf. AG-80, $\$ 617$ und $\S 628$ ), und sie einer einzigen Wortbildungskategorie zuordnen.

Viele Wortbildungsparadigmen enthalten neben solchen allgemeinen Beziehungsadjektiven weitere, die das semantische Verhältnis zwischen ihrem Basiswort und dem dazugehörigen Substantiv nach verschiedenen Richtungen hin spezifizieren:

a) Eindeutig metonymische Beziehungsadjektive. Es handelt sich um zusammengesetzte Adjektive mit verbaler Zweitkomponente, die eine bestimmte situative Beziehung ausdrückt. In unserem Material kommt lediglich vinogradouboročnyj vor.

b) Eindeutig metaphorische Beziehungsadjektive. Hier sind in erster Linie die Adjektive auf -vidnyj, -podobnyj, -obraznyj zu nennen, bei denen die Form der Frucht 
als Vergleichsgrundlage dient: gruševidnyj, grušeobraznyj, tykvoobraznyj, tykvopodobnyj. Der Bezug auf die Farbe kann durch Komposita ausgedrückt werden, in denen das Beziehungsadjektiv mit einem richtigen Farbadjektiv verbunden wird: limonno-želtyj, cf. brusnično-krasnyj. (So enthält BAS2 auch zu granatovyj die Bedeutungsangabe 'imejuščij cvet granata [1. Granat vo 2 znač.]; temno-krasnyj'; im einzigen Beleg, den das Wörterbuch dazu liefert, kommt jedoch das Adjektiv in Verbindung mit krasnyj vor: "[Illjuminator], kak blestjaščij zagadočnyj glaz, granatovo-krasnyj i prozračnyj".) Zusammengesetzte Adjektive wie das rein scherzhafte seroburo-malinovyj verlangen ebenfalls nach einer metaphorischen Deutung. Die Form der Blätter dient als tertium comparationis im fachsprachlichen rjabinolistnyj, das aus unerfindlichen Gründen in BASI (und ORF) aufgenommen wurde.

$\mathrm{Zu}$ den Adjektiven, die eine Ähnlichkeitsbeziehung ausdrücken, muß schließlich limonnokislyj gestellt werden, bei dem der Geschmack der Ptlanze im Vordergrund steht. Allerdings sind die Verhältnisse hier komplizierter, da diesem Adjektiv in Wirklichkeit nicht das Beziehungsadjektiv limonnyj, sondern die Mehrwortbenennung limonnaja kislota zugrundeliegt. Limonnokislyj ist also von dem Grundwort limon durch ein lexikalisiertes (wenn auch polylexematisches) substantivisches $Z$ wischenglied getrennt und dürfte deshalb strenggenommen gar nicht in dessen Wortbildungsparadigma aufgenommen werden. Um es jedoch guten Gewissens auszuschließen, müßten wir über eindeutige Kriterien zur Abgrenzung der einfachen Verbindungen zwischen Beziehungsadjektiv und Substantiv von den lexikalisierten Fügungen des Typs limonnaja kislota verfügen. Wie wir bereits oben bei der Behandlung der Univerbierungen bemerkt haben, ist die Aufstellung solcher Kriterien alles andere als trivial; wir ziehen es deshalb vor, den problematischen Begriff der Mehrwortbenennung (sostavnoe naimenovanie) in unseren Beschreibungsapparat erst gar nicht einzuführen und dafür eine gewisse Unsauberkeit in der Behandlung von limonnokislyj in Kauf zu nehmen. (Einen ähnlichen Fall stellt unter den Ableitungen der Kategorie ovošč (Gemüse) das Adjektiv ščavelevokislyj dar.)

Bei den metaphorischen Beziehungsadjektiven ist ein in vielen Wortbildungsparadigmen wiederkehrender Gegensatz zwischen Farb- und Formadjektiven festzustellen. Dieser Gegensatz tritt nicht nur auf der semantischen, sondem auch auf der formalen und pragmatischen Ebene auf:

- die Formadjektive verfügen über ein eigenes Inventar von Wortbildungsmitteln (-podobnyj, -vidnyj, -obraznyj, -čatyj), die Farbähnlichkeit wird durch die allgemeinen Suffixe -ov- und - $n$ - ausgedrückt;

- den Farbadjektiven kommt primär eine charakterisierende (limonnaja perčatka, smorodinnye glaza), den Formadjektiven eine nominativ-taxinomische Funktion zu (gruševidnaja ajva, limonovidnaja dynja); 
- metaphorische Fügungen, in denen die Farbe der Frucht als tertium comparationis dient, gehören meist dem literarischen Stil an. Sie werden von den Muttersprachlern als dichterisch empfunden, während die Fügungen mit den Formadjektiven eher zur Fachsprache tendieren: man vergleiche etwa in dieser Hinsicht brusničnyj zakat mit limonovidnaja dynja.

Diese Beobachtungen sprechen dafür, für die metaphorischen Formadjektive auf -vidnyj, -obraznyj und -podobnyj eine eigene Wortbildungskategorie aufzustellen. Damit enthält die adjektivische Zone unseres Wortbildungsparadigmas folgende Kategorien:

a) Allgemeine Beziehungsadjektive (adj): abrikosnyj, abrikosovyj, ajvovyj, ananasnyj, ananasovyj, apel'sinnyj, apel'sinovyj, arbuznyj, bananovyj, čerešnevyj, dynnyj, eževičnyj, eževikovyj, granatovyj, granatnyj, gruševyj, inžirnyj, jabločnyj, klubničnyj, kokosovyj, kryžovennyj, kryžovnikovyj, limonno-želtyj, limonnokislyj, limonnyj, limonovyj, sero-buro-malinovyj, malinno-zemljaničnyj, malinnyj, malinovyj, mandarinnyj, mandarinovyj, mangovyj, ovoščnoj, persikovyj, rjabinnyj, rjabinovyj, slivnyj, slivovyj, smorodinnyj, smorodinovyj, černosmorodinnyj, černosmorodinovyj, ternovyj, tykvennyj, vinogradouboročnyj, vinogradnyj, višnevyj, temnovišnevyj, visennyj, zemljaničnyj.

(Ableitungen wie malinno-zemljaničnyj oder černosmorodinovyj stehen natürlich nicht auf der ersten Ableitungsstufe; ersteres wird aufgenommen, weil adjektivische Zwischenglieder den Ausschluß eines Wortes aus dem Wortbildungsparadigma nicht rechtfertigen, letzteres, weil in unserem Modell kein Platz für Mehrwortbenennungen wie černaja smorodina als ableitende Wörter ist.)

b) Metaphorische Beziehungsadjektive, die sich auf die Form der Frucht beziehen (mph): gruševidnyj, grušeobraznyj, limonovidnyj, tykvopodobnyj, tykvoobraznyj. Das fachsprachliche rjabinolistnyj 'ebereschenblättrig' ist am besten auch in diese Kategorie einzuordnen, obwohl hier die Form der Blätter und nicht die Frucht den Vergleichsgegenstand abgibt.

Verben oder Adverbien sind in den Wortbildungsparadigmen der Kategorie frukty (Obst) nicht vertreten.

In welchem Maße werden nun die aufgestellten Wortbildungskategorien den Forderungen des Kap. 3.1 gerecht? Die Forderung der Ökonomie wird vor allem durch AUG und COL verletzt, die in unseren Wortbildungsparadigmen jeweils nur durch ein Exemplar vertreten sind und deshalb von vielen anderen Kategorien durch keine Opposition abgegrenzt werden können (bei AUG kann man nur eine Opposition zu DEM aufstellen, da das Wortbildungsparadigma von arbuz, in dem das Augmentativum arbuzišče vorkommt, keine weiteren Ableitungen in der substantivischen Zone 
enthält). Von den Oppositionen, die man mit den übrigen Wortbildungskategorien bilden kann, kann lediglich FEM -- GEN nicht belegt werden (die Opposition jabloćnica :: jablonevye ist wegen des Zwischenglieds jablonja problematisch). Selbstverständlich ist dies auf eine zufällige Lücke in unserem Korpus und nicht auf eine semantische oder formale Verwandtschaft zwischen diesen beiden Kategorien zurückzuführen.

Schlechter ist es um die Vollständigkeit unserer Beschreibung bestellt: die beiden Paare rjabinuška :: rjabinka und limonen :: limonad müßten strenggenommen zur Aufspaltung der Kategorien DEM und RES führen. Der Gewinn an Vollständigkeit, der sich daraus ergäbe, würde freilich durch die groben Verletzungen des Ökonomieprinzips durch die neuen, eingliedrigen Kategorien von rjabinuška und limonen mehr als aufgehoben; und im Fall von limonen darf man sich fragen, ob dieses Wort überhaupt in das Wortbildungsparadigma von limon hineingehört. Sonstige Mehrfachableitungen in einer Wortbildungskategorie sind entweder synonym (slivjanka/slivovica); oder aber sie betreffen Komposita (vinogradouboročnyj vs. vinogradnyj) bzw. natural kinds (zahlreiche Beispiele in der Kategorie TAX). Auf die spezielle Problematik der Kategorie LOC und der Beziehungsadjektive wurde bereits oben eingegangen.

Die meisten der aufgestellten Wortbildungskategorien genügen der Forderung der Natürlichkeit; einzig und allein die Restkategorie der Gegenstandsbezeichnungen (RES) stellt eine Ausnahme dar.

Am Ende dieses Kapitels wollen wir kurz auf die Erscheinung der sog. hyponymischen Motivation eingehen. Es handelt sich hierbei um Ableitungen, in denen das Basissubstantiv eine ihm übergeordnete semantische Kategorie vertritt. In unserem Material findet sich dafür unter anderem das Beispiel limonad, das im MAS als "sladkij proxladitel'nyj bezalkogol'nyj napitok s sokom limona ili drugix fruktov $i$ jagod" [unsere Unterstreichung] erklärt wird; ein Beispiel aus einer anderen Kategorie wäre ložkomojka, das von ložka 'Löffel' abgeleitet ist, aber praktisch dieselbe Bedeutung wie posudomojka 'Tellerwäscherin' hat. Das Phänomen der hyponymischen Motivation wurde von E.A. ZEMSKAJA ins Gespräch gebracht und in einer ganzen Reihe von Publikationen unter den verschiedenen Motivationstypen angeführt (siehe ZEMSKAJA 1981: 137, ZEMSKAJA 1984: 340sq., wo noch der Terminus periphere Motivation gebraucht wird, sowie ERMAKOVA/ZEMSKAJA 1991: 107). In KAKRIDIS 1993 wird der Versuch unternommen, das Zustandekommen hyponymisch motivierter Ableitungen unter Rückgriff auf die Prototypensemantik plausibel zu machen.

Konsequenterweise müßte man dem Begriff der hyponymischen Motivation den der hyperonymischen Motivation an die Seite stellen und Ableitungen wie gruśnik 'Verkäufer getrockneter Birnen' auf dieselbe Weise wie limonad analysieren. 
Im Rahmen einer paradigmatischen Wortbildungsanalyse können nur Bedeutungsunterschiede, die sich in einer (oder mehreren) formalen Opposition manifestieren, bei der Aufstellung der Wortbildungsbedeutung berücksichtigt werden. Dies ist aber sogar in einem so klar ausgeprägten Beispiel wie limonad nicht der Fall: limonad ist von limonovka nicht (nur) durch die Erweiterung im Geltungsbereich des Grundwortes, sondern durch die Merkmale 'einheimisch, oft hausgemacht' getrennt, auf die wir wegen solcher Paare wie abrikotin :: abrikosovka nicht verzichten können. Die Bedeutungskomponente 'drugie frukty i jagody', die in die lexikalische Bedeutung von limonad eingeht, ist kein Bestandteil der Wortbildungskategorie, der dieses Wort angehört.

\subsubsection{Ausgewählte Wortbildungsparadigmen}

GRUŠA 'Birnbaum; Birne; birnenförmiges Gerät, Ballon, z.B. Zerstäuber, Autohupe u.ä.'

DEM gruška 'Dem. zu gruša'

TAX grušovka 'eine Art süßer, mehliger Äpfel, Birnapfel'

HOM grušnik 'Verkäufer von gedämpften oder getrockneten Birnen'

LOC gruševina 'Birnbaum'

LOC grušina 'Birnbaum'

LOC grušica 'Birnbaum'

LOC grušovnik 'Birngarten'

LIQ grušovka 'Birnwein, Bimbranntwein'

RES grušovnik 'Bimenkwas'

mph gruševidnyj 'birnenförmig'

mph grušeobraznyj 'bimenförmig';

adj gruševyj 'Birnen-, Bimbaum-'

LIMON 'Zitronenbaum, Zitrone'

DEM limončik 'Dem. zu limon'

TAX limonka 'eine Art Birnen, deren Form an eine Zitrone erinnert' 'prost. zitronenförmige Handgranate' (BAS-1)

TAX limonnik 'bot. Schizandra'

TAX limonnica 'Gonepteryx rhamni, ein Schmetterling der Familie der Kohlweißlinge mit zitronengelben Flügeln beim Männchen'

LOC limonarij 'Pflanzstätte für Zitronen'

LIQ limonovka 'Zitronenbranntwein' 
RES limonad 'Limonade'

RES limonen 'das Limonen, 1-Methyl-4-isopropylzyklohexadien-(1,8)'

adj limonno-želtyj 'zitronengelb'

adj limonnokislyj 'Zitronensäure enthaltend'

adj limonnyj 'Zitronen-'

adj limonovyj 'Zitronen-'

mph limonovidnyj 'zitronenförmig'

TYKVA 'Kürbis'

DEM tykovka 'Dem. zu tykva'

GEN tykvennye 'Cucurbitaceae, Kürbisgewächse'

LOC tykvennik 'Kürbisgewächse'

RES tykvennik 'Kürbisbrei, Kürbisgrütze'

SIN tykvina 'Kürbiskopf'

adj tykvennyj 'Kürbis-'

mph tykvopodobnyj 'kürbisförmig'

mph tykvoobraznyj 'kürbisförmig'

VINOGRAD 'Weinrebe, Weinstock; Weintrauben'

TAX vinogradovnik 'Ampelopsis'

GEN vinogradnye 'Vitaceae'

GEN vinogradovye 'Vitaceae'

HOM vinogradar' 'Winzer, Weinbauer'

LOC vinogradnik 'Weinberg'

RES vinogradarstvo 'Weinanbau, Wissenschaft vom Weinanbau'

SIN vinogradina '(einzelne) Weinbeere'

SIN vinogradinka '(einzelne) Weinbeere'

adj vinogradouboročnyj 'Weinlese-'

adj vinogradnyj 'Wein-'

Als letztes führen wir die Wortbildungsparadigmen von frukt und ovošc an, die auf einer höheren Kategorisierungsstufe als die übrigen hier behandelten Substantive stehen: 


\section{FRUKT 'Obst'}

HOM fruktovščik 'Obstverkäufer'

HOM fruktovar 'Arbeiter, der das Kochen von Obst beaufsichtigt'

RES fruktovoz 'Kühlschiff zum Transport von Obst'

RES fruktoprovod 'Rohrleitung zum Transport von Früchten mit Hilfe von Wasser'

RES fruktoxranilišče 'Speziallager für Obst'

RES fruktoza 'Fruchtzucker'

RES fruktofuranozidaza 'chem. Verbindung'

RES suxofrukty 'Trockenfrüchte'

adj fruktovyj 'Obst-'

adj fruktovo-jagodnyj 'Obst- und Beeren-'

adj fruktopererabatyvajuščij 'obstverarbeitend'

\section{OVOŚC 'Gemüse'}

TAX plodoovošč 'Obst und Gemüse'

HOM ovoščevod 'Gemüseanbauer'

HOM ovoščnik 'Gemüseverkäufer'

FEM ovoščevodka 'Gemüseanbauerin'

RES ovoščesušilka 'Gemüsetrocknungsanlage'

RES ovoščerezka 'Gemüseschneidemaschine'

RES ovoščevodstvo 'Gemüseanbau'

RES ovoščexranilišče 'Speziallager für Gemüse'

RES ovoščebaza 'Ort, wo Gemüse gelagert und verarbeitet wird'

adj ovoščnoj 'Gemüse-'

kartofel'no-ovoščnoj 'Kartoffel- und Gemüse-'

kartofeleovoščnoj 'Kartoffel- und Gemüse-'

plodovo-ovoščnoj 'Früchte- und Gemüse-'

ovošče-baxčevoj 'Gemüse- und Melonen-'

ovoščesušil'nyj 'Gemüsetrocken-'

ovoščemoečnyj 'Gemüsewasch-'

ovoščevodčeskij 'Gemüseanbau-'

ovoščekartofelevodčeskij 'Gemüse- und Kartoffelanbau-'

ovoščekonservnyj 'Gemüsekonserven-'

ovoščemoločnyj 'Gemüse- und Milch-'

ovoščepererabatyvajuščij 'gemüseverarbeitend'

ovoščeprigotovitel'nyj 'Gemüsezubereitungs-'

ovoščerezatel'nyj 'Gemüseschneide-' 
Obwohl alle Ableitungen dieser zwei Wortbildungsparadigmen ohne weiteres in eine der aufgestellten Wortbildungskategorien eingeordnet werden können und -- falls man -vod und -var als Zweitglieder von Komposita und nicht als Suffixoide ansieht -- das Vollständigkeitsprinzip der Beschreibung durch ihre Eingliederung in das abstrakte Wortbildungsparadigma der Kategorie frukty (Obst) nicht verletzt wird, fallen doch einige deutliche Unterschiede ins Auge: DEM fehlt, TAX (bis auf das Kompositum plodoovošc) ebenfalls; dafür sind RES, HOM und die zusammengesetzten Adjektive in großer Anzahl vertreten. Als Gesamteindruck ergibt sich eine größere Nähe der Ableitungen dieser Wortbildungsparadigmen zum wissenschaftlichen und zum publizistischen Funktionalstil.

\subsection{Analyse der Wortbildungsparadigmen der Kategorie Ovošči (Gemüse)}

\subsubsection{Zum Bestand der Kategorie}

Der Geltungsbereich des abstrakten Wortbildungsparadigmas, das (re)konstruiert werden soll, ist durch die Substantive vorgegeben, die in unserem Produktionsexperiment als Mitglieder der Kategorie ovošči genannt wurden; zwei davon, arbuz und tykva, wurden sowohl in der Kategorie frukty als auch in der Kategorie ovošči aufgeführt ( 27 vs. 2 bzw. 1 vs. 16 Nennungen). Wir lassen die Mehrwortbenennungen zelenyj gorošek, kapusta kvašenaja und cvetnaja kapusta beiseite und sehen in Fällen wie morkov/morkovka, redis/rediska, kartofel'kartoška, repa/repka beide Wörter als gemeinsamen Ausgangspunkt des Wortbildungsparadigmas an (cf. Anm. 2 unseres Wortbildungsalgorithmus). Zelen', das zwar Hyponym von ovošci ist (cf. MAS s.v. ovošci), aber auf einer anderen Kategorisierungsstufe steht als die übrigen Substantive, soll getrennt analysiert werden. Die Ergebnisse des Experimentes von V. N. MASKADYNJA enthalten lediglich die zusätzlichen Substantive bazar, ogorod, pole, deren Nennung auf einer Kontiguitätsassoziation beruht; sie brauchen hier nicht berücksichtigt zu werden. Die Liste, die R. M. FrUMKINA und ihre Mitarbeiter ihren Experimenten zugrundegelegt haben (FRUMKINA/MIXEEV/MOSTOVAJA/RJUMINA 1991: 65) können wir nicht heranziehen; die Gründe dafür wurden bereits oben bei der Analyse der Wortbildungsparadigmen der Kategorie frukty (Obst) dargelegt.

\subsubsection{Zum Bestand der Wortbildungsparadigmen}

Auch in dieser Kategorie sind die Substantive polysem: mit ihnen kann sowohl auf die lebende Pflanze Bezug genommen werden, als auch auf jenen Teil, der gegessen wird. Die Gegenüberstellung ist jedoch weniger deutlich ausgeprägt als bei den Mitgliedern der Kategorie frukty (Obst) -- tatsächlich kann man sich fragen, ob es sich um echte Polysemie oder bloßes highlighting handelt --, und viele Ableitungen sind nur willkürlich der einen oder der anderen Bedeutungsvariante zuzuordnen. Wir werden deshalb von einer Aufspaltung der Wortbildungsparadigmen absehen. 


\subsubsection{Das abstrakte Wortbildungsparadigma der Kategorie Ovošči (Gemüse)}

Die substantivische Zone umfaßt folgende Wortbildungskategorien:

a) Deminutiva (DEM): arbuzik, brjukovka, buračok, česnočok, gorošek zu gorox [auch für die unreifen, süßen Erbsenkörner, die als Beilage oder zum Würzen verwendet werden; in dieser Bedeutung TAX], kapustka, kartošečka, kartofel'ka, lučok, ogurčik, perčik, pertrušečka, pomidorčik, redisočka, redečka, salatik, svekolka, tykovka, ukropec, ukropčik.

Viele dieser Ableitungen entwickeln auch eine singulative Bedeutung.

b) Augmentativa (AUG): arbuzišče, xrenovina (in der Bedeutung 'große Meerrettichwurzel').

c) Singulativa (SIN): bobok, bobina, bobinka, brjukvina, gorošina, gorošinka, kartošina, kartofelina, kartofelinka, lukovica, lukovina, lukovka, perčinka, pomidorina, pomidorka, repina, tykvina.

Mit diesen Ableitungen kann der Sprecher auf eine einzelne Knolle, Erbse etc. Bezug nehmen; die vermittelnde Assoziation ist jedoch stark metonymisch geprägt, indem von der Pflanze aus die Bezeichnung für einen ihrer Teile gebildet wird. In dieser Hinsicht stehen diese Bildungen den Ableitungen der Kategorie RES nahe, mit denen sie auch formale Ähnlichkeiten aufweisen (cf. goroxovina, xrenina).

d) Taxinomische Ableitungen (TAX): bobki 'Lorbeeren', bobovye 'Hülsenfruchtgewächse', zernobobovye 'Hülsenfruchtgewächse', brjukvennica 'Pieris napi, Schmetterling aus der Familie der Weißlinge (Pieridae), deren Raupen vornehmlich auf der Steckrübe und auf anderen Arten der Kreuzblütler leben', buračnik 'der Boretsch, das Gurkenkraut', česnočnik 'Blätter-, Knoblauchpilz', česnočnica 'Knoblauchkröte', goroxovik 'Caraganda arborescens, der sibirische Erbsen-, Schotenbaum', goroxovnik 'Caraganda arborescens, der sibirische Erbsenbaum', 'Lathyrus, die Platterbse', 'das Muschelblümchen, die Tolldocke', gorošek 'Wicke (bot.); Tupfen, Pünktchen (als Stoffmuster)', kapustnik 'Kohlraupe', kapustnica 'Kohlweißling', luk-batun oder batunluk 'Allium fistulosum', šnitt-luk oder luk-šnitt 'Allium schoenoprasum, Schnittlauch', šalot-luk oder luk-šalot 'Allium Ascalonicum, Aschlauch', morkovina 'Mohrrübenkraut', morkovnik 'Mohrrübenkraut', ogurečnik 'Gurkenkraut' 'eine Fischart aus der Familie der Osmeridae', perečnik 'Bauernsenf; Pfefferkraut', percejad 'Pfefferfresser, Tukan', perečnye 'Piperaceae', repnica 'Pieris rapae' zool., repnik 'Rapistrum, Rübenpflanze', surepa 'Rübsamen; Barbenkraut', surepica id., surepka id., repovnik id., repej 'Klette', repejnik 'Klette', rep'e 'Klette', rep’jak 'Klette', sveklovica 'Zuckerrübe', 
podsvekol'nik 'Amarantus retroflexus, bogiger Fuchsschwanz', tykvennye 'Cucurbitaceae, Kürbisgewächse'.

Dies ist eine sehr reiche und in sich nicht besonders einheitliche Kategorie. Neben Pflanzennamen, die die Mehrzahl der Ableitungen ausmachen, kommen auch Tiernamen vor; die vermittelnde Assoziation ist metaphorischer (česnočnica), gelegentlich auch metonymischer Natur (brjukvennica). Viele Wortbildungsparadigmen weisen mehrere Ableitungen in dieser Kategorie auf; da es jedoch allesamt natural kinds sind, braucht keine weitere Differenzierung vorgenommen zu werden.

e) Personenbezeichnungen (HOM): kartofelevod 'Spezialist für Kartoffelanbau', lukovod 'Spezialist für Zwiebelanbau', ogurečnik 'Gurkenmann', redečnik 'Freund von Rettich, Rettichhändler, Rettichpflanzer', sveklovod 'Spezialist für Rübenanbau'.

Eine Differenzierung nach Geschlecht oder nach fach- und gemeinsprachlichen Bildungen (lukovod vs. ogurečnik) erweist sich als überflüssig.

f) Gegenstandsbezeichnungen mit lokativer Komponente (LOC): goroxovišče 'das gewesene Erbsenfeld', kapustnik 'Kohlgarten, Kohlfeld', kartofljanišče 'gewesenes Kartoffelfeld', ogurečnik 'Gurkenbeet', repišče 'Rübenfeld', sveklovišče 'Zuckerrübenfeld', sveklosovxoz 'Sowjetgut, auf dem Rüben angebaut werden', tykvennik 'Kürbisgewächse', xrenovnik 'Meerrettichbeet'.

Einige dieser Ableitungen enthalten das Sem 'abgeerntet' (kartofljanišče, goroxovišče), das jedoch niemals in differenzierender Funktion auftritt. Wie in der Kategorie frukty (Obst) haben einige Ableitungen auf -nik eine kollektive Nebenbedeutung (tykvennik).

g) Gegenstandsbezeichnungen mit situativer Komponente (SIT): česnočnica 'hölzemer Mörser zum Zerstoßen des Knoblauchs', kapustnica 'Kohlschneidetag' 'Kübel zur Aufbewahrung von Kohl', kartofelezagotovka 'Kartoffelbeschaffung', kartofelekombajn 'Kartoffelkombine', kartofelekopalka 'Kartoffelroder', kartofelekopatel' 'Kartoffelroder', kartofelemojka 'Kartoffelwaschmaschine', kartofelepečka 'Kartoffelbackgerät', kartofelesażalka 'Kartoffelpflanzmaschine', kartofelesažatel' 'Kartoffelpflanzmaschine', kartofelesortirovka 'Kartoffelsortiermaschine', kartofeleterka 'Kartoffelreibe', kartofelexranilišče 'Kartoffelmiete', kartofelečistka 'Kartoffelschälmaschine', kartofelevodstvo 'Kartoffelanbau', lukovodstvo 'Zwiebelanbau', lukorezka 'Zwiebelschneidemaschine', perečnica 'Pfefferbüchse', salatnik 'Salatschüssel', salatnica 'Salatschüssel', sveklovodstvo 'Zuckerrübenbau', sveklokombajn 'Rübenerntemaschine, Rübenkombine', sveklokopatel' 'Rübenheber', sveklomojka 'Rübenwäsche, Rübenwaschmaschine', sveklopogruzčik 'Rübenlader', sveklopod-emnik 'Rübenheber', 
sveklorezka 'Rübenschneidemaschine', sveklosejanie 'Rübensaat', sveklouborka 'Rübenernte', svekloutomlenie 'Rübenmüdigkeit'.

In den Komposita zeigt das Zweitglied die zugrundeliegende Situation an. Doch gehören auch einige suffixale Ableitungen zu unserer Kategorie: man vergleiche in dieser Hinsicht lukorezka und česnočnica (beide Gegenstände dienen zum Zerkleinern der Nahrungsmittel), kapustnica (als Bezeichnung für einen Kübel, in dem man Kohl aufbewahrt) und kartofelexranilišče etc.

h) Gegenstandsbezeichnungen mit rein metonymischer Bedeutung (Teil-GanzesBeziehungen) (RES): goroxovina 'der Erbsenstengel mit Blättern, das Erbsenstroh', repnjak 'das Kraut, die Blätter der Rübenpflanze', sveklosemena 'Rübensaatgut', svekol'nik 'Rübenkraut, Blätter der Rübe', xrenina 'Meerrettichwurzel'.

i) Bezeichnungen für Gerichte und Getränke (ALI): goroxovica 'Erbsensuppe', gorošnica 'Erbsensuppe, Erbsenbrei', goroxovik '1, der Erbsenkuchen, Aschkuchen aus Erbsenmehl (in Gestalt eines abgestumpften Kegels); 2, eine Pirogge mit Erbsenfüllung', kapustnik 'Kohlpastete', kartofel'nik 'Kartoffelpirogge, Kartoffelpastete' 'Kartoffelspeise', morkovnik 'Mohrrübensuppe, Mohrrübenpastete', percovaja 'Pfefferbranntwein, Schnapsaufguß auf Pfeffer', percovka 'Pfefferbranntwein, Schnapsaufguß auf Pfeffer', svekol'nik 'Rübensuppe', tomatoprodukty 'Tomatenprodukte', tomat-pasta 'Tomatenpaste', tomat-pjure 'Tomatenpüree', tykvennik 'Kürbisbrei, Kürbisgrütze'.

Von diesen Kategorien verletzt nur eine, AUG, das Ökonomieprinzip; alle übrigen stehen in mindestens einem Wortbildungsparadigma zueinander in Opposition. Das Vollständigkeitsprinzip wird in den Kategorien DEM (ukropec vs. ukropčik) und ALI (goroxovica/gorośnica vs. goroxovik) verletzt; diese Ableitungen stehen jedoch einander so nahe, daß sie kaum die Aufstellung eigenen Kategorien rechtfertigen -zumal diese Kategorien unweigerlich die Forderung nach Beschreibungsökonomie verletzen würden. Alle aufgestellten Kategorien erlauben eine natürliche semantische Interpretation.

Die adjektivische Zone des Wortbildungsparadigmas soll hier -- anders als in der Kategorie frukty (Obst) -- nicht weiter aufgeteilt werden. Da rein metaphorische Adjektive auf -vidnyj, -podobnyj und -obraznyj nicht sehr zahlreich sind und sie auch als Komposita interpretiert werden können, werden sie zusammen mit allen anderen denominalen Adjektiven zur Kategorie adj zusammengefaßt. Eine interessante Opposition zwischen zwei suffixalen Ableitungen kommt im Wortbildungsparadigma von perec vor: percovyj, perečnyj 'Pfeffer-' vs. perčenyj 'gepfeffert'. Da die ornative Bedeutung von perčenyj jedoch auch bei percovyj und perečnyj als eine Möglichkeit unter anderen vorhanden ist (cf. die Erklärungen in USA: 's percem') und kein anderes Wortbildungsparadigma ein entsprechendes Adjektiv aufweist, ist die Opposi- 
tion zu schwach, um die Aufstellung einer eigenen Kategorie zu rechtfertigen. Aus der Fülle der Beziehungsadjektive, die von Substantiven der Kategorie ovošči abgeleitet werden, führen wir nur einige Beispiele an: arbuznyj, baklažannyj, fasolevyj, fasoleuboročnyj, goroxovyj, goroxovidnyj...

Das Wortbildungsparadigma von perec enthält auch die einzige verbale Ableitung von einem Substantiv der Kategorie ovošči (Gemüse): perčit:

4.2.4 Ausgewählte Wortbildungsparadigmen

KAPUSTA 'Kohl'

DEM kapustka 'Dem. zu kapusta'

ALI kapustnik 'Kohlpastete'

TAX kapustnik 'Kohlraupe'

TAX kapustnica 'Kohlweißling'

LOC kapustnik 'Kohlgarten, Kohlfeld'

SIT kapustnica 'Kohlschneidetag'; 'Kübel zur Aufbewahrung von Kohl'

adj kapustouboročnyj 'Kohlernte-'

adj kapustnyj 'Kohl-'

\section{LUK 'Zwiebel'}

DEM lučok 'Dem. zu luk'

SIN lukovica 'Knoblauch- oder Zwiebelknolle'

SIN lukovina 'Knoblauch- oder Zwiebelknolle'

SIN lukovka 'Knoblauch- oder Zwiebelknolle'

HOM lukovod 'Spezialist für Zwiebelanbau'

SIT lukovodstvo 'Zwiebelanbau'

SIT lukorezka 'Zwiebelschneidemaschine'

TAX luk-batun 'Allium fistulosum', auch in der Form batun-luk

TAX šnitt-luk 'Allium schoenoprasum, Schnittlauch', auch in der Form luk-šnitt

TAX salot-luk 'Allium Ascalonicum, Aschlauch', auch in der Form luk-šalot

adj lukovyj 'Zwiebel-'

adj lukosejuščij 'Zwiebelanbau-'

adj lukouboročnyj 'Zwiebelernte-' 
SVEKLA 'Rübe'

TAX sveklovica 'Zuckerrübe'

LOC sveklovišče 'Zuckerrübenfeld'

HOM sveklovod 'Spezialist für Rübenanbau'

SIT sveklovodstvo 'Zuckerrübenanbau'

SIT sveklokombajn 'Rübenemtemaschine, Rübenkombine'

SIT sveklokopatel' 'Rübenheber'

SIT sveklomojka 'Rübenwäsche, Rübenwaschmaschine'

SIT sveklopogruzčik 'Rübenlader'

SIT sveklopod-emnik 'Rübenheber'

SIT sveklorezka 'Rübenschneidemaschine'

RES sveklosemena 'Rübensaatgut'

SIT sveklosejanie 'Rübensaat'

LOC sveklosovxoz 'Sowjetgut, auf dem Rüben angebaut werden'

SIT sveklouborka 'Rübenernte'

SIT svekloutomlenie 'Rübenmüdigkeit'

DEM svekolka 'Dem. zu svekla'

ALI svekol'nik 'Rübensuppe'

RES svekol'nik 'Rübenkraut, Blätter der Rübe'

TAX podsvekol'nik 'Amarantus retroflexus, bogiger Fuchsschwanz'

adj sveklovodčeskij 'Zuckerrübenanbau-, für Zuckerrübenanbau'

adj svekol'nyj 'Rüben-'

adj sveklopriemočnyj 'Rübenabnahme-'

adj sveklosaxarnyj 'Zuckerrüben-'

adj sveklosejuščij 'Rüben anbauend'

adj sveklouboročnyj 'Rübenemte-'

Als letztes wollen wir das Wortbildungsparadigma von zelen vorstellen; es fällt insofern aus der Reihe, als in ihm die einzige Opposition zwischen einer männlichen (bzw. neutralen) und einer weiblichen Personenbezeichnung belegt ist -- was allerdings wieder auf die Unvollständigkeit unseres Korpus zurückzuführen sein wird. Man kann nämlich ohne weiteres weibliche Gegenstücke zu der Kategorie HOM bilden; die Opposition HOM -- FEM fehlt in den bisher beschriebenen Wortbildungsparadigmen vor allem deshalb, weil die Bedeutungsangaben der russischen Wörterbücher unsystematisch bzw. unvollständig sind: man vergleiche PAW, der z.B. zu kapustnik die Bedeutungen 'ein Freund von Kohl, der Kohlesser; der Kohlgärtner, -verkäufer' und zu kapustnica 'eine Freundin von Kohl, die Kohlesserin; die Kohlgärtnerin, -verkäuferin' anführt. 
ZELEN' 'Gemüse, Grünzeug'

HOM zelenščik 'Verkäfer von Grünzeug, Gemüse'

FEM zelenščica 'Fem. zu zelenščik'

adj zelennoj 'Gemüse-'

\subsection{Analyse der Wortbildungsparadigmen der Kategorie Pticy (Vögel)}

\subsubsection{Zum Bestand der Kategorie und der Wortbildungsparadigmen}

Für diese Kategorie steht uns neben den Ergebnissen unseres eigenen Experimentes lediglich die Liste von V. N. MASKADYNJA zur Verfügung. Letztere bietet folgende Ergänzungen: filin, burevestnik, gagara, krylo, kulik, peresmešnik, polet, orlan, staja. Davon sind die auf Kontiguitätsassoziation beruhenden Nennungen von krylo, polet und staja auszuschließen. Ferner lassen wir die Binomina (ptica-sekretar) und die Mehrwortbenennungen (volnistyj popugajčik) aus unseren Betrachtungen aus.

Die Wortbildungsparadigmen dieser Kategorie wurden in der (leider ungedruckten) Dissertation von N. G. JUSUPOVA untersucht. JUSUPOVA hat durch Auswertung eines Materialkorpus weitaus mehr Ableitungen gefunden, als in den Wörterbüchern des Russischen verzeichnet sind. Einige dieser Ableitungen werden im folgenden unserem Material hinzugefügt; bei der Aufstellung unserer Wortbildungskategorien beschränken wir uns jedoch auf Ableitungen, die im SSS belegt sind.

\subsubsection{Das abstrakte Wortbildungsparadigma der Kategorie Pticy (Vögel)}

In der substantivischen Zone finden sich folgende Wortbildungskategorien:

a) Deminutiva (DEM): capel'ka, čižik, cyplenoček, cypa, fazančik, galočka, gagarka, gluxarek, golubok, goluboček, golubčik, gusek, jastrebok, kanareečka, koršunok, kuličok, kukušica, kukušečka, kurka, kuročka, kuropatočka, lebedka, lebeduška, orlik, penočka, petušok, popugajčik, sinička, skvorka, skvoruška, snegirek, sokolik, solovejko, solovejčik, solovejuška, solovušek, solovuška, sovka, strižok, utočka, vorobus̉ek, vorobejčik, voronuška, voronka, żuravel'čik, żuravlik, żuravuška.

b) Pejorativa (PEJ): petušiška, kurčonka.

c) Augmentativum (AUG): utišča.

Sehr häufig werden Vogelbezeichnungen morphologisch abgewandeh, um neue Denotate zu bezeichnen -- sei es in derselben (orel $\rightarrow$ orlan) oder in einer anderen 
Kategorie (jastreb $\rightarrow$ jastrebok). Die vermittelnde Assoziation ist meist metaphorischer Natur; aber in kanareečnik 'Kanariengras' liegt eine metonymische Verbindung vor (cf. die russische Bedeutungserklärung 'travjanistoe rastenie sem. zlakov, semena kotorogo ispol'zujutsja kak korm dlja komnatnyx ptic [gl. obrazom kanareek]'). Schließlich verfügt die biologische Fachsprache über eine eigene Wortbildungskategorie, um terminologische Bezeichnungen für übergeordnete Taxa zu bilden.

d) Taxonyme (TAX): aistnik 'Geranium, Storchschnabel', galočka 'Markierungszeichen in Form eines fliegenden Vogels [ ]', grifon 'Greif (myth.)', gusek 'mehrere taxinomische Bedeutungen, darunter: Hohlkehle (am Gesims), Rinnleiste', gusjatnik 'Bezeichnungen für mehrere Pflanzen, u.a. Gänsekraut', jastrebok 'Typus eines Jagdflugzeugs', kanareečnik 'Kanariengras', kuročka 'Wasserhuhn, gemeines Teichhuhn', lebedka 'liegende Winde, Kurbelwelle', podorlik 'Aquila clanga', orlan 'Seeadler', orljak 'Alpengeier', pavlinoglazka 'Vanessa Io, Tagpfauenauge', sovka 'Zwergohreule', utkonos 'Ornithorhynchus, Schnabeltier', žuravel'nik 'Geranium, Kranich-, Storchschnabel', žuravec 'Brunnenschwengel; Hebebaum, Kran'.

e) Biologische Taxonyme (GEN): aistovye, aistoobraznye, čajkovye, djatlovye, djatloobraznye, drozdovye, fazanovye, gagarovye, golubinye, golubeobraznye, grifovye, gusinye, guseobraznye, jastrebinye, kukuškoobraznye, kurinye, pelikanovye, sinicevye, slavkovye, sokolinye, sokoloobraznye, trjasoguzkovye, utinye, vorobinye, voronovye (zu voron und vorona).

Eine Zwischenstellung zwischen den metonymischen und den metaphorischen Ableitungen nehmen die Bezeichnungen für Vogeljunge, Vogelweibchen und Vogelmännchen ein:

f) Vogeljunge (FIL): aistenok, [čižonok JuSUPOVA 1980: 54], drozdenok, fazanenok, filinenok, galčonok, [gagarenok JUSUPOVA 1980: 54], gluxarenok, golubenok, gračenok, gusenok, gusenyš, indjušonok, jastrebenok, koršunenok, kuličonok, kuličenok, kukušonok, kurčonok, kurenok, lebedenok, orlenok, perepelenok, pingvinenok, [rjabčonok JUSUPOVA 1980: 52], ščeglenok, skvorčonok, snegirenok, sokolenok, solov'enok, [soročonok JUSUPOVA 1980: 54], sovenok, strausenok, strižonok, syčonok, teterevenok, utenok, utenyš, vorob'enok, voronenok (zu voron und vorona), žavoronoćek, [žavorenok JUSUPOVA 1980: 52], žuravlenok.

g) Vogelweibchen (FEM): fazanixa, fazanka, gluxarka, golubka, golubuška, golubica, gusynja, guska, klestovka, [kuličixa JusuPOVA 1980: 42], orlica, pava, perepelka, perepelica, [rjabčixa JUSUPOVA 1980: 46], ščeglixa, ščeglovka, skvorčixa, skvorka, snegirixa, sokolixa, sokolica, sokolena, solovka, solovixa, teterka, tetera, vorobixa, vorobka, voronixa, żuravlixa. 
h) Vogelmännchen (MAS): indjuk, kur, gusak.

Schließlich finden wir ein breitgefächertes Inventar an rein metonymischen Ableitungen:

i) Vogelfleisch (CAR): cypljatina, fazanina, gluxarjatina, golubjatina, gusjatina, indjušatina, kurjatina, lebedjatina, lebjažina, [petušatina JuSUPOVA 1980: 61], utjatina, [żuravljatina JUSUPOVA 1980: 61].

j) Gefäß, in dem der Vogel zubereitet wird (VAS): gusjatnica, utjatnica.

k) Vogelkäfige, -gehege und dgl. (LOC): cypljatnik, fazanarij, golubjatnja, golubjatnik, gračovnik, gusjatnik, gusjatnja, gusarnja, indjušatnik, kurjatnik, kurnik (mit der isolierten Bedeutung 'Hühnerpastete' neben 'Hühnerstall'), kurjatnja, (popugajnik JUSUPOVA 1980: 64], siničnik, skvorečnik, skvorećnica, skvorečnja, strausjatnik.

1) (Männliche) Lebewesen, die in einer metonymisch-situativen Beziehung zum Vogel stehen: ihn jagen, (traditionell) züchten etc. (SAM): cypljatnik 'Hühnerhabicht', golubjatnik 'Taubenliebhaber; Taubenfalk, -habicht', golubec 'Taubenhabicht', gusjatnik 'Gänsehändler, -freund, -hirt; großer Hühnerhabicht', jastrebjatnik, kanareečnik, kurjatnik 'Hühnerhabicht', kuroed id., kurocap id., perepeljatnik 'Sperber, Finkenhabicht', ščegljatnik 'Liebhaber von Stieglitzen', skvorečnik 'Liebhaber von Staren', sokol'nik 'Falkenjäger, Falkner', sokol'ničij 'Oberfalkenmeister (als Hofamt)', sokoljatnik 'Liebhaber von Falken', teterevjatnik 'Birkhahnhabicht', utjatnik, utjatnica, vorobjatnik 'Wachtelfalk'.

m) Die weibliche Entsprechung zu j): cypljatnica, gusjatnica, kurjatnica, skvorećnica.

n) Personen, die sich auf die moderne, wissenschaftliche Vogelzucht spezialisiert haben (VOD): fazanovod, golubevod, gusevod, kurovod.

o) Moderne, wissenschaftliche Vogelzucht (ART): fazanovodstvo, golubevodstvo, gusevodstvo, indejkovodstvo, kurovodstvo, popugajstvo, utkovodstvo.

Metonymisch ist schließlich wohl auch die Kategorie der Kollektiva zu interpretieren; die beste Übersetzung der beiden einzigen Ableitungen dieser Kategorie ins Deutsche wäre "Rabenschwarm, Geierschwarm". Beide Ableitungen weisen eine ausgeprägt pejorative Nuance auf.

p) Kollektiva (KOL): vorone, koršune.

Die meisten der aufgestellten Wortbildungskategorien genügen der Forderung nach Vollständigkeit der Analyse: wo ein Wortbildungsparadigma mehrere Ableitungen 
enthält, die unter dieselbe Wortbildungskategorie fallen, so sind diese entweder synonym (gusenok, gusenyš; lebedjatina, lebjažina; sokolixa, sokolica; etc.) oder von der Forderung nach Vollständigkeit ausgenommen, weil in allen Oppositionen, die man mit ihnen bilden kann, mindestens ein Oppositionsglied ein natural kind oder ein Kompositum ist (orlan, orljak; golubec, golubjatnik; kurjatnik, kuroed, kurocap etc.). Nur bei den Deminutiva haben wir, wie auch in den übrigen Kategorien, darauf verzichtet, Mehrfachableitungen auf unterschiedliche Kategorien zu verteilen -- nicht aus prinzipiellen Erwägungen wie bei den Komposita und den natural kinds, sondern weil diese Aufgabe auf der Grundlage unseres Materials einfach nicht zu bewältigen ist.

Auch die Natürlichkeit unserer Wortbildungskategorien kann u.E. nicht in Frage gestellt werden. Problematisch ist lediglich die Beschreibungsökonomie: die drei Kategorien PEJ, AUG und COL sind weder gegeneinander noch gegen einer Reihe weiterer Kategorien durch Oppositionen abgesichert. Man könnte diesem Mangel abhelfen, indem man alle drei Kategorien zu einer einzigen zusammenfaßt. Angesichts der Tatsache, daß allen drei Wortbildungskategorien eine gewisse pejorative Nuance gemeinsam ist, ist diese Lösung weniger unnatürlich, als es auf den ersten Blick scheint.

In der adjektivischen Zone des Wortbildungsparadigmas kommen wir mit zwei Wortbildungskategorien aus: einer sehr allgemeinen (adj), deren Mitglieder wie immer in einzelnen Fügungen die unterschiedlichsten Beziehungen zum nachfolgenden Substantiv ausdrücken können (sokolinaja oxota, sokolinye oči, kurinyj bulon, golubinyj pomet, lebedinaja šeja), und einer, die die mit dem Suffixoid -vodčeskij gebildeten Adjektive umfaßt (art): golubevodčeskij, gusevodčeskij, indejkovodčeskij, kurovodčeskij.

Die verbale und die adverbiale Zone umfassen je nur eine Wortbildungskategorie -kein Wortbildungsparadigma enthält mehr als ein nichtsynonymes Verb oder Adverb:

a) Verben (ver): pavlinit'sja 'sich wie ein Pfau brüsten', petušitsja 'hitzig sein wie ein Hahn', popugajničat' 'wie ein Papagei nachplappem', sorocit' 'wie eine Elster plappern, schwatzen'.

b) Adverbien (adv): po-fazani 'nach Fasanenart', po-gusinomu 'nach Gänseart', gusem 'im Gänsemarsch', gus'kom id., po-jastrebinomu, po-koršuni, po-kuriči, po-kurinomu, po-lebedinomu, po-orlinomu, po-petušinomu, po-petuši, petuškom, popugajski, posokolinomu, po-soroči, po-utinomu, po-vorobinomu, po-voroni, po-voron'emu, pożavoronočì, po-žuravlinomu.

Es ist bemerkenswert, daß sowohl den Verben als auch den (äußerst zahlreichen) Adverbien der Wortbildungsparadigmen der Kategorie pticy (Vögel) ausnahmslos 
metaphorische Assoziationen zugrundeliegen. Man vergleiche damit die Wortbildungsparadigmen der Kategorie posuda (Küchengeschirr und Besteck), in denen die Verben metonymische Assoziationen der vielfältigsten Art (Kasusrollen!) zum Ausdruck bringen -- bis auf štoporit', das bezeichnenderweise ein Vergleichsadverb neben sich hat: štoporom. Die Analogie zwischen Vogel und Mensch, die in diesen Ableitungen zum Ausdruck kommt, ist in vielen Kulturen produktiv (zu der Welt der Vögel als einer "société humaine métaphorique" cf. die feinen Beobachtungen bei LÉvi-STRAuSS 1962: 270sq.).

Die metaphorische Grundbedeutung des Deminutivs bringt es mit sich, daß ihm bei der Ableitung dieser Adverbien oft der Vorzug gegeben wird: cf. guskom, petuškom etc. (cf. SARNOWSKI 1991: 12, § 4.5.1. Zur komparativen Bedeutung, die Adverbien auf -o unter dem Einfluß der Adverbien auf po-...-omu entwickeln, cf. PANOV 1968, III: 250sqq.).

In den Wortbildungsparadigmen der Kategorie pticy (Vögel) kommen schließlich auch zwei Interjektionen vor: uti-uti und cyp-cyp.

\subsubsection{Ausgewählte Wortbildungsparadigmen}

FAZAN 'Fasan'

DEM fazančik 'Dem. zu fazan'

FEM fazanixa 'Fasanenweibchen'

FEM fazanka 'Fasanenweibchen'

FIL fazanenok 'Fasanenjunges'

CAR fazanina 'Fasanenfleisch'

VOD fazanovod 'Fasanenzüchter'

ACT fazanovodstvo 'Fasanenzucht'

GEN fazanovye 'Fasanartige'

LOC fazanarij 'Fasanengehege, -zwinger'

adj fazanij 'Fasanen-'

adj fazanovyj 'Fasanen-'

adv po-fazani 'nach Fasanenart'

OREL 'Adler'

DEM orlik 'Dem. zu orel'

TAX podorlik 'Aquila clanga'

TAX orlan 'Seeadler' 
TAX orljak 'Alpengeier'

FEM orlica 'Adlerweibchen'

FIL orlenok 'Adlerjunges'

adj orlij 'Adler-'

adj orlinyj 'Adler-'

adj orlenyj 'Adler-'

adv po-orlinomu 'nach Adlerart'

UTKA, UTICA, UTJA 'Ente'

DEM utočka 'Dem. zu utka'

AUG utišča 'Aug. zu utka'

GEN utinye 'Entenvögel'

TAX utkonos 'Schnabeltier'

FIL utenok 'Entenjunges'

FIL utenys 'Entenjunges'

CAR utjatina 'Entenfleisch'

SAM utjatnik 'Entenhabicht'

MEF utjatnica 'Frau, die die Enten füttert'

VAS utjatnica 'Gefäß zum Braten von Enten'

ACT utkovodstvo 'Entenzucht'

adj utinyj 'Enten-'

adj utjačij 'Enten-'

adj utkonosyj 'Entenschnabel-'

adv po-utinomu 'nach Entenart'

int uti-uti 'Ruf, mit dem man Enten anlockt'

4.4 Analyse der Wortbildungsparadigmen der Kategorie Posuda (Küchengeschirr und Besteck)

\subsubsection{Zum Bestand der Kategorie}

Wir besitzen zu dieser Kategorie drei Listen von Mitgliedern: die Liste, die R. M. FrUMKINA und ihre Mitarbeiter benutzt haben (FrUMKINA/MiXEEV/MOSTOVAJA/RJUMINA 1991: 66), die Ergebnisse unseres Experimentes sowie die Ergebnisse des Experimentes von V. N. MASKADYNJA. Unsere Versuchspersonen haben folgende Kategorienmitglieder genannt, die in der Liste von FrUMKINA et al. fehlen: serviz, salatnik, skorovarka, supovnik, stolovaja ložka, serviz obedennyj, serviz čajnyj, servetka, podstavka, piala, čerpak, čan, amfora. Dazu kommen skovorodka (bei 
FRUMKINA: skovoroda), vazočka (bei FRUMKINA: vaza) und čugun (bei FRUMKINA: cugunok).

Von diesen Bezeichnungen kommen serviz čajnyj und serviz obedennyj wegen ihrer morphologischen Struktur (Mehrwortbenennungen) für unsere Analyse nicht in Betracht. Zwei weitere (supovnik, servetka) sind weder bei SSS noch bei TIX bezeugt, so daß über deren Wortbildungsparadigma keine Aussagen getroffen werden können. Bei skovoroda/skovorodka und čugun/čugunok fassen wir nach Anm. 2 unseres Wortbildungsalgorithmus beide Lexeme zu einem zusammen; vazočka wird neben vaza in unsere Ausgangsliste aufgenommen, hat allerdings ein leeres Wortbildungsparadigma.

V. N. MASKADYNJA benutzte als Stimulus nicht posuda, sondern die Umschreibung prinadleżnost dlja edy und erhielt eine Liste, die neben Geschirrbezeichnungen auch Wörter wie moloko, mjaso oder xolodil'nik enthielt. Die neuen Kategorienmitglieder sind: salfetka, čajnaja ložka, paločki (japonskie, kitajskie), povareška, lavrovyj list, moloko, mjaso, ovošc̆, paločki (buterbrodnye), pogreb, frukt, xolodil'nik, posuda. Wir können davon nur salfetka und posuda (unter Vorbehalt) in den Geltungsbereich unseres abstrakten Wortbildungsparadigmas einschließen.

Damit enthält unsere Ausgangsliste alle Substantive, die bei FRUMKINA/MIXEEv/MOSTOVAJA/RJUMINA 1991: 66 aufgeführt sind (cf. FRUMKINA/MIKHEJEV 1996: 115 sq., mit der richtigen Form suxarnica statt suxarnica für $\mathrm{Nr}$. 57), sowie folgende Erweiterungen: amfora, čan, čerpak, piala, podstavka, posuda, povareška, salatnik, salfetka, serviz, skorovarka, vazočka.

\subsubsection{Zum Bestand der Wortbildungsparadigmen}

Manche Wortbildungsparadigmen unserer Kategorie weisen Ableitungen auf, in die das Grundwort nicht in seiner Funktion bei der Nahrungszubereitung und -aufnahme eingeht: kotel'naja 'pomeščenie, $v$ kotorom ustanovleny parovye kotly' (MAS) (cf. kotlopunkt in NS-2: 'punkt kotlovogo pitanija (prigotovlenija gorjačej pišči v kotlax|') oder ponožovščina 'draka s primeneniem nożej' (cf. nožetočka 'prisposoblenie dlja točki kuxonnyx nożej [s pomoščju zatočnyx plastin]' in NS-2). Daneben existieren Ableitungen, auf die die Mehrdeutigkeit des Grundwortes vererbt wurde: kotlovoj (davlenie, dovol'stvie) oder noževoj (nadrez, rana). Wir werden die entsprechenden Wortbildungsparadigmen aufspalten, da Ableitungen wie die soeben zitierten nicht mehr auch nur im entferntesten mit der Situation der Nahrungszubereitung und -aufnahme verbunden sind. 
4.4.3 Das abstrakte Wortbildungsparadigma der Kategorie Posuda (Küchengeschirr und Besteck)

Die substantivische Zone weist folgende Wortbildungskategorien auf:

a) Deminutiva (DEM): banočka, bidončik, bljudečko, bokalčik, butyločka, čajniček, čašečka, fljažka, fljažečka, goršoček, kastrjul'ka, kofejniček, kotel'čik, kotelok, kovšik, kovšiček, kružečka, kuvšinec, ložečka, misočka, nožik, nožiček, pestik, podnosik, rešetco, rjumočka, rjumaška, salfetočka, sitce, škal'čik, skaločka, skovorodka, stakašek, stakančik, štofec, štofik, štofčik, stopočka, štoporik, stupka, sudoček, tareločka, teročka, vazočka, viločka, żarovenka.

b) Augmentativa (AUG): goršišče, čugunišče, kotlišče, kružišča, nožišče, stopar', stopak.

Der Bereich der taxinomischen Wortbildung ist reich vertreten; wir finden hier in erster Linie Ableitungen, die unter dieselbe Kategorie wie das Ausgangswort fallen (bljudo $\rightarrow$ bljudce, vaza $\rightarrow$ vazon), aber auch Bezeichnungen für Mitglieder anderer Kategorien -- Tiere (butylkonos), Pflanzen (kuvšinka), geographische Formationen (kotlovina) und Werkzeuge (nožovka). Auf der Ausdrucksseite gibt es wie immer zahlreiche Überlappungen mit der expressiven Wortbildung. In einer Reihe von Fällen hat ein ursprüngliches Deminutiv die Oberhand gewonnen und das Wort, von dem es abgeleitet wurde, in eine stilistisch und semantisch periphere Position abgedrängt: so ist butylka gebräuchlicher als butyl', čaška als čaša etc. Wie bereits in Kapitel 2 dargelegt, sind die formale und die inhaltliche Beziehung zwischen den beiden Wörtem hier gegenläufig: die Ausdrucksseiten von butyl' und čaša sind in den Ausdrucksseiten von butylka und časka enthalten, aber im inneren Aufbauf der Kategorie posuda nehmen butylka und čaška eine zentralere Stellung ein, so daß sie als Bezugspunkte für die Einordnung von butyl und čaša dienen. Unsere Entscheidung, sowohl čaša in das Wortbildungsparadigma von čaška aufzunehmen als auch umgekehrt, trägt dieser Unbestimmtheit Rechnung.

Die Opposition zwischen von Menschenhand hergestellten und in der Natur vorgefundenen Gegenständen kehrt in einer Reihe von Wortbildungsparadigmen wieder und findet in kotlovan vs. kotlovina formalen Ausdruck. Wir stellen deshalb zwei Wortbildungskategorien auf -- eine für Artefakte (ART) und eine für Naturgegenstände (NAT). Eine weitere Systematisierung des Materials ist nicht möglich, so daß Mehrfachbelegungen dieser Kategorien in mehreren Wortbildungsparadigmen vorkommen.

c) Taxinomische Ableitungen (Artefakte, ART): banočka 'Schnapsglas', banki 'Schröpfkopf', bljudo 'Schüssel' (zu bljudce), bljudce 'Untertasse' (zu bljudo), butyl' 'große Flasche, Korbflasche', pol(u)butylka 'halbe Flasche', [čaška 'Tasse, Schüssel, 
Nut'; zu čăa, das nicht zu unseren Ausgangssubstantiven gehört], čaša 'Kelch' (zu čaška), kotelok 'Napf, Feldkessel; steifer Hut, Glocke', kotlovan 'Baugrube', ložki 'Löffel (als Musikinstrument)', misa 'Suppenschüssel, Terrine', nożnicy 'Schere', noženki 'Dem. zu nožnicy'3, nožovka 'Fuchsschwanz, Bogensäge', èlektroskovoroda 'elektrische Pfanne', pol(u)štof '1/2 Eimer', stopa 'großer Pokal, Humpen, Becher', sudki 'Einsatz von Schüsseln (zum Holen des Essens)', tarel '(veraltet) Teller', tarelki 'Schallbecken', tareločka 'Zielscheibe', vazon 'dekorativer Blumentopf', vily 'Heugabel'.

d) Taxinomische Ableitungen (Naturgegenstände, NAT): bljudce 'Bodensenkung in der Steppe', butylkonos', čašečka 'Blütenkelch, Kniescheibe', kotelok 'Kopf', kotlovina 'Kesselschlucht', ložečka, podložka 'Herzgrube's, ložečnica 'Löffelkraut', nožekljuv 'Scheren, Verkehrtschnabel', pestik '(bot.) Stempel, Pistill', sitovina 'vermodertes, mürbes od. von Würmern zerfressenes Holz; morscher, fauler, ausgehöhlter Baum', viločka 'Ausschnitt, Vertiefung des Brustbeins (anat.)'.

In den übrigen Wortbildungskategorien ist die Beziehung zwischen dem ableitenden und dem abgeleiteten Wort metonymischer Natur:

e) Männliche bzw. unmarkierte Personenbezeichnungen (HOM): lizobljud 'Kriecher', bljudoliz id., lizobljudnik id. ${ }^{6}$, sobutyl'nik 'Zechbruder, Saufkumpan', butyločnik 'Flaschenhersteller', čašnik 'Mundschenk; Kellermeister', goršenja 'Töpfer', goršečnik id., kastrjul'nik 'Arbeiter, der die Töpfe wäscht; Tellerwäscher', kotel'nik 'Kesselschmied', kotel'ščik id., ložečnik 'Hersteller von Holzlöffeln', ložkar' id., misočnik 'Schüsselverfertiger', nožovščik 'Messerschmied', rešetnik 'Siebmacher'.

${ }^{3}$ Da nožnicy zu noženki nicht in einer echten Inklusionsbeziehung steht, kann es nach unseren Regeln aus dem Wortbildungsparadigma von nož nicht ausgeschlossen werden.

4 Zur metaphorischen Interpretation von butylkonos 'Entenwal, Dögling (Hyperoodon ampullatus)' cf. die Erklärung in der BSĖ2: "golova zakančivaetsja butylkoobrazno suživajuščimsja rylom, otkuda i proizošlo nazvanie 'B/utylkonos]'". Strenggenommen sind solche Ableitungen zweistufig, indem ihnen sowohl eine metonymische (Benennung des Tieres nach einer seiner Gliedmaßen) als auch eine metaphorische (Vergleich dieser Gliedmaße mit einem Artefakt) Assoziation zugrundeliegt; cf. nožekljuv. Führt man das Wort auf nesti zurück, wie es TIX tut, so muß man es natürlich metonymisch interpretieren.

s Cf. IOMDIN 1996: 371 zur strukturgleichen Bildung podmyška.

${ }^{6}$ Diese Wörter werden heute nur in übertragenem Sinn gebraucht. 
Manche dieser Ableitungen bezeichnen Personen, die den betreffenden Gegenstand herstellen (goršečnik), andere bezeichnen Personen, die ihn benutzen (sobutyl'nik) oder reinigen (kastrjul'nik). Die Opposition zwischen 'benutzen' und 'herstellen' ist nur ansatzweise belegt: in der scherzhaften und auch formal abweichenden Bildung sobutyl'nik, das zu butyločnik in Opposition steht. Ein weiteres Paar wäre ponožovščik $\because$ nožovščik, doch wird in ponožovščik 'Messerstecher' das Messer bestimmt nicht als Mitglied der Kategorie posuda empfunden.

f) Weibliche Personenbezeichnungen (FEM): goršečnica, kastrjul'nica, ložkomojka, nožovščica.

g) Lokative Gegenstandsbezeichnungen (LOC), d.h. Bezeichnungen für Gegenstände, die zur Aufnahme des Gegenstandes dienen, der vom ableitenden Wort denotiert, wird: podbljudnik 'Unterschale, Schüsselring', misočnik 'Untersatz zur Suppenschüssel', nožny 'Scheide', podstakannik 'Teeglaseinsatz'.

h) Situative Gegenstandsbezeichnungen (SIT): fljagomojka 'Kanisterspülmaschine', kotlopunkt 'Feldküche', ložkomojka 'Gefäß zum Waschen des Geschirrs', nožetočka 'Gerät zum Schärfen von Messern', rjumočnaja 'Branntweinschenke', sitnik 'Brot aus gebeuteltem Mehl', sitnyj id., štofnaja 'Branntweinschenke', vazopis' 'Vasenmalerei'.

Das ableitende und das abgeleitete Wort bezeichnen Gegenstände, die unter verschiedenen Funktionen in die Grundsituation eingehen. Wenn man die Kasusrollen affiziertes Objekt (AOB), effiziertes Objekt (EOB), Instrument (INS) und Ort (LOC) differenziert, so kommen folgende Konstellationen vor:

AOB - INS: fljagomojka, fljagoproparivatel', ložkomojka, nožetočka

INS - LOC: kotlopunkt, rjumočnaja, štofnaja

INS - EOB: sitnik, sitnyj

Schließlich bezeichnet eine Ableitung keinen Gegenstand, sondern eine Tätigkeit; das ableitende Wort bezeichnet das effizierte Objekt: vazopis'.

Es ist nun wichtig festzuhalten, daß diese Kasusrollen mit den Mitteln der paradigmatischen Wortbildungsanalyse nicht differenziert werden können: dazu bräuchten wir Wortbildungsparadigmen, in denen mehrere affixale Ableitungen mit unterschiedlichen Kasusrollenkonstellationen nebeneinander vorkommen. Solche Wortbildungsparadigmen sind in unserem Material nicht belegt.

i) Gegenstandsbezeichnungen ohne lokative oder situative Komponente (RES): bidonvil' 'Slum mit Hütten, die aus Blechkanistern gebaut sind', čašelistik 'Kelchblatt' 
(zu čaška und čaša; semantisch natürlich zu čašečka in der Bedeutung 'Blütenkelch'), noževišče 'Messerschaft', skovorodnik 'Pfannengabel'.

Dies ist eine inhomogene Restkategorie; noževišče und skovorodnik enthalten das gemeinsame Sem 'Griff', das jedoch nicht in die Wortbildungsbedeutung aufgenommen werden kann, da es im rein partitiven cašelistik und im stark idiosynkratischen Lehnwort bidonvil' fehlt.

Unser abstraktes Wortbildungsparadigma genügt der Forderung der Ökonomie: jede der aufgestellten Wortbildungskategorien steht zu jeder anderen in mindestens einem Wortbildungsparadigma in Opposition. Dies ist trivialerweise schon deshalb der Fall, weil im Wortbildungsparadigma von nož alle Kategorien vertreten sind; freilich bezeichnet dieses Substantiv einen Gegenstand, dessen Funktionen weit über die Kategorie posuda hinausreichen, und Ableitungen wie nožny oder nožovka lassen bestimmt nicht in erster Linie an die Situation der Nahrungszubereitung und -aufnahme denken; bei nožny liegt überdies hyponymische Motivation vor, die die Ableitungsbeziehung dieses Wortes zu nož weiterhin abschwächt: in der Kategorie der Substantive, die durch nožny geschützt werden, ist nož selbst kein zentrales Mitglied (cf. KAKRIDIS 1993). Läßt man nož beiseite, so lassen sich besonders unter den schwächer belegten Kategorien kaum Oppositionen ausfindig machen:

\begin{tabular}{||l|l|l|l|l|l||}
\hline & AUG & FEM & RES & LOC & SIT \\
\hline AUG & & belegt & $?$ & $?$ & belegt \\
\hline FEM & belegt & & $?$ & belegt & $?$ \\
\hline RES & $?$ & $?$ & & $?$ & $?$ \\
\hline LOC & $?$ & belegt & $?$ & & $?$ \\
\hline SIT & belegt & $?$ & $?$ & $?$ & \\
\hline
\end{tabular}

Wie die Tabelle zeigt, könnte man, wenn man das Tripel

$$
\text { noževišče }:: \text { nožny }:: \text { nožetočka }
$$

unberücksichtigt ließe, RES, LOC und SIT zu einer Wortbildungskategorie zusammenfassen. Diese Kategorie würde alle metonymischen Ableitungen enthalten, die keine Personenbezeichnungen sind, d.h. nur unbeseelte Substantive umfassen. Bei den Augmentativa und den weiblichen Personenbezeichnungen ist zu berücksichtigen, daß unser Korpus sicher nicht alle Bildungen umfaßt, die in diesem Bereich möglich sind; die Anzahl der Oppositionen in den einzelnen Wortbildungsparadigmen ließe sich wohl noch vergrößern. 
Weitaus schlechter ist es um die Vollständigkeit unserer Beschreibung bestellt: nichtsynonyme Mehrfachableitungen kommen in den Kategorien DEM, ART und NAT in großer Anzahl vor. DEM könnte man in zwei oder sogar drei (cf. das Tripel štofec $::$ štofik $::$ śtofčik) (Unter)kategorien aufspalten -- mit entsprechenden Einbußen bei der Ökonomie der Beschreibung und mit der Schwierigkeit, jedes Deminutiv einer der so entstandenen Kategorien zuordnen zu müssen. Eine solche Zuordnung scheint ohne Auswertung eines größeren Materialkorpus nicht möglich zu sein; allein nach der Gestalt des Suffixes darf man jedenfalls nicht vorgehen, wie z.B. vazočka zeigt, das kein vazka neben sich hat und wohl nicht mit kastrjulečka, sondern mit kastrjul'ka auf dieselbe Stufe zu stellen ist.

Einige der aufgestellten Kategorien verletzen schließlich die Forderung der Natürlichkeit: die Zusammenfassung von skovorodnik und bidonvil in RES bzw. podstakannik und nožny in LOC liefert die zwei extremsten, aber ganz gewiß nicht die einzigen Beispiele für solche Verletzungen.

Geht man die aufgezeigten Mängel einzeln durch, so scheinen sie leicht zu beheben zu sein. Man überzeugt sich jedoch sehr bald, daß jedes weitere Zugeständnis an eines der drei Analyseprinzipien (z.B. die Zusammenfassung von skovorodnik und noževišče zu einer separaten Wortbildungskategorie, die eine weitaus natürlichere semantische Interpretation als die Kategorie RES zuließe, etwa 'Griff von...') mit einer Verletzung eines der beiden anderen erkauft werden muß (im angeführten Beispiel ist dies das Prinzip der Ökonomie). Unser abstraktes Wortbildungsparadigma ist der erträglichste Kompromiß, den man erreichen kann; er zeigt, daß die Annahme, den konkreten Wortbildungsparadigmen der Kategorie posuda liege ein einziges abstraktes Wortbildungsparadigma zugrunde, keine besonders hohe Plausibilität für sich beanspruchen darf -- soweit man dies bei der allgemein geringen Wortbildungsproduktivität ihrer Mitglieder überhaupt entscheiden kann. Der Grund dafür ist am ehesten darin zu sehen, daß die fragliche Kategorie nicht nur durch Ähnlichkeits-, sondern auch durch Kontiguitätsassoziationen konstituiert wird; für die Konstruktion des abstrakten Wortbildungsparadigmas kommt jedoch nur die Parallelität zwischen den einzelnen konkreten Wortbildungsparadigmen in Betracht, nicht die Tatsache, daß einige dieser Wortbildungsparadigmen weitere Kategorienmitglieder enthalten (auch diese Fälle sind übrigens recht selten und durchaus nicht immer durch Kontiguitätsassoziationen vermittelt, wie der Vergleich von bljudce mit podbljudnik zeigt).

Bei der Betrachtung der adjektivischen Zone unserer Wortbildungsparadigmen stellen wir fest, daß die meisten Ableitungen je nach dem Substantiv, mit dem sie verbunden werden, die verschiedensten Beziehungsarten zum Ausdruck bringen können:

tareločnaja sušilka (cf. fljagomojka)

tareločnaja čečevica (cf. ložečnica)

sitnaja muka (cf. sitnik) 
ložečnoe proizvodstvo (cf. vazopis)

Wo es um den eindeutigen Ausdruck eines bestimmten situativen Zusammenhangs geht, werden Komposita gebildet: čašeobraznyj, fljagomoečnyj, podbljudnyj. Nur in einem einzigen Fall stehen zwei verschiedene suffixale Ableitungen zueinander in Opposition: tarel'čatyj (cf. bokal'čatyj) vs. tareločnyj. Allerdings zeigt die soeben zitierte Fügung tareločnaja čečevica, daß auch tareločnyj Ähnlichkeitsbeziehungen ausdrücken kann, so daß die Opposition auf semantischer Seite nicht besonders ausgeprägt ist. Da jedoch zum Ausdruck von Ähnlichkeitsbeziehungen im Russischen spezialisierte, quasisuffixale Wortbildungsmittel zur Verfügung stehen (-vidnyj, -obraznyj), wollen wir der Vollständigkeit Vorrang vor der Ökonomie geben und zwei Wortbildungskategorien aufstellen: mph für Beziehungsadjektive, die auf Ähnlichkeitsbeziehungen festgelegt sind, und adj für alle übrigen. Es sei übrigens angemerkt, daß die meisten Beziehungsadjektive der Kategorie posuda recht künstlich klingen und nur im technischen Bereich gebräuchlich sind.

a) adj: banočnyj, žestjanobanočnyj, bidonnyj, podbljudnyj, butyločnyj, butylomoečnyj, butyločnozelenyj, čašečnyj, fljažnyj, fljagomoečnyj, goršečnyj, kastrjul'nyj, kotel’nyj, kotlovyj, kružečnyj, kuvšinnyj, ložečnyj, misočnyj, noževoj, pestovyj, podnosnyj, posudnyj, posudoxozjajstvennyj, posudoštampovočnyj, posudomoečnyj, rešetnyj, rjumočnyj, salfetočnyj, serviznyj, sitnyj, škaličnyj, skovorodnyj, stakannyj, štofnyj, štopornyj, sudkovyj, tareločnyj, teročnyj, termosnyj, vazovyj, vertel'nyj, viločnyj, žarovennyj.

b) mph: bokal'čatyj, bokalovidnyj, butyločnozelenyj, [čaševidnyj, čašeobraznyj zu $\check{c}$ aša ], kotloobraznyj, nożevidnyj, nožeobraznyj, sitovidnyj, štoporoobraznyj, tarel'čatyj, tarelkoobraznyj.

In der verbalen Zone kommen folgende Ableitungen vor:

sobutyl'ničat' 'zechen'

ćašničat' 'zechen'

goršečničat' 'das Töpferhandwerk ausüben, Töpfe herstellen'

ložkarit' 'sich mit der Verfertigung hölzerner Löffel beschäftigen'

rešetit" 'durchsieben'

śtoporit' 'trudeln'

Wir stellen hier dieselbe Vielfalt wie bei SIT fest, brauchen aber nicht mehr als eine Wortbildungskategorie (ver) aufzustellen, da kein einziges Wortbildungsparadigma mehr als eine verbale Ableitung enthält.

Ein einziges Wortbildungsparadigma enthält ein (metaphorisches) Adverb: štoporom 'spiral-, schraubenförmig'. 
4.4.4 Ausgewählte Wortbildungsparadigmen

BUTYLKA 'Flasche'

DEM butyločka 'Dem. zu butylka'

NAT butylkonos 'Entenwal, Dögling'

ART butyl' 'große Flasche, Korbflasche'

ART pol(u)butylka 'halbe Flasche'

HOM sobutyl'nik 'Zecher'

FEM sobutyl'nica 'Zecherin'

HOM butyločnik 'Flaschenhersteller'

adj butyločnyj 'Flaschen-'

adj butylomoečnyj 'Flaschenspül-'

mph butyločnozelenyj 'flaschengrün'

ver sobutyl'ničat' 'zechen'

KOTEL 'Kessel'

DEM kotel'čik 'Dem. zu kotel'

DEM kotelok 'Dem. zu kotel'

AUG kotlišče 'Aug. zu kotel'

ART kotelok 'Napf, Feldkessel; steifer Hut, Glocke'

NAT kotelok 'Kopf'

ART kotlovan 'Baugrube'

NAT kotlovina 'Kesselschlucht'

HOM kotel'nik 'Kesselschmied'

HOM kotel'ščik 'Kesselschmied'

SIT kotlopunkt 'Feldküche'

mph kotloobraznyj 'kesselförmig'

adj kotel'nyj 'Kessel-'

adj kotlovyj 'Kessel-'

LOŽKA 'Löffel'

DEM ložečka 'Dem. zu ložka'

NAT ložečka 'Herzgrube'

NAT podložka 'Herzgrube'

NAT ložečnica 'Löffelkraut'

ART ložki 'Löffel (als Musikinstrument)'

HOM ložečnik 'jemand, der (Holz)löffel anfertigt' 
HOM ložkar' 'jemand, der (Holz)löffel anfertigt'

FEM ložkomojka 'Frau, die Löffel etc. spült'

SIT ložkomojka 'Ort, wo Löffel etc. gespült werden'

adj ložečnyj 'Löffel-'

ver ložkarit' 'sich mit der Herstellung von (Holz)löffeln beschäftigen'

NOŽ 'Messer'

DEM nožik 'Dem. zu noż'

DEM nožiček 'Dem. zu noż'

AUG nožišče 'Aug. zu nož'

ART nożnicy 'Schere'

ART noženki 'Dem. zu nožnicy'

ART nožovka 'Fuchsschwanz, Bogensäge'

NAT nożekljuv 'Scherenschnabel'

RES noževišče 'Messergriff'

LOC nožny 'Scheide'

HOM nožovščik 'Messerhersteller'

FEM nožovščica 'weibliche Entsprechung zu nožovščik'

SIT nožetočka 'Gerät zum Wetzen von Messern'

mph noževidnyj 'messerförmig'

mph nožeobraznyj 'messerförmig'

adj noževoj 'Messer-'

Wir führen schließlich noch das Wortbildungsparadigma des Substantivs posuda an, das auf einer höheren Kategorisierungsstufe steht, aber von einigen Informanten von MASKADYNJA unter prinadleżnost dlja edy genannt wurde:

POSUDA 'Geschirr'

SIN posudina 'einzelnes Geschirrstück'

SIN posudinka 'einzelnes Geschirrstück'

LOC posudnik 'Küchenschrank, Regal zur Aufbewahrung von Geschirr'

HOM posudnik 'Tellerwäscher'

FEM posudomojka 'Tellerwäscherin'

FEM posudomojščica 'Tellerwäscherin; Frau, die eine Geschirrspülmaschine bedient'7

${ }^{7}$ Cf. die Bedeutungserklärung in NS-2: 'mojščica posudy na predprijatii obščestvennogo pitanija; posudomojka' und den Beleg: "podxodim k posudomoečnoj mašine... P. stavit grjaznye stakany i tarelki na polki, a s drugogo konca mašiny oni 
SIT posudomojka 'Geschirrspülmaschine; Ort, an dem Geschirr gespült wird' adj posudnyj 'Geschirr-'

adj posudoxozjajstvennyj 'Geschirr- und Haushalts-'

adj posudoštampovočnyj 'Geschirrstanz-'

adj posudomoečnyj 'Geschirrspül-'

Das Wortbildungsparadigma weist bis auf die beiden Singulativa posudina und posudinka dieselben Wortbildungskategorien wie die Wortbildungsparadigmen der nächstniedrigeren Kategorisierungsstufe auf.

\subsection{Analyse der Wortbildungsparadigmen der Kategórie Sredstva peredviženija (Fortbewegungsmittel)}

Zu der Kategorie Fortbewegungsmittel liegen uns die Ergebnisse unseres eigenen Experimentes und des Experimentes von V. N. MASKADYNJA vor. Die Durchsicht dieser Listen fördert ein beträchtliches Ausmaß an semantischer und morphologischer Heterogenität zutage; wir finden hier

- einen größeren Anteil an abgeleiteten Substantiven als in den übrigen Kategorien: samolet, paroxod, èlektrička etc.;

- Fremdwörter, die -- anders als bei den bisher untersuchten Kategorien -- nicht nur periphere, sondern auch zentrale Kategorienmitglieder bezeichnen: avtobus, metro, velosiped etc.;

- abstrakte Substantive (beg, ezda, xod'ba);

- ein Adverb: peškom;

- Bezeichnungen für landwirtschaftliche Geräte (interessanterweise nur von den Versuchspersonen in Kalinin/Tver' genannt): traktor, kombajn; für Körperteile: noga; für Tiere: osel, kon', lošad', verbljud;

Einen zusätzlichen Heterogenitätsfaktor stellt die Tatsache dar, daß viele Fortbewegungsmittel für sportliche und/oder militärische Zwecke genutzt werden können; bei

vyxodjat čistymi". Das semantische (nicht jedoch das formale) Verhältnis zwischen posudomojka (als Gerätebezeichnung) und posudomojščica ist mit Paaren wie sterilizator -- sterilizatorščik, pitatel' -- pitatel'ščik zu vergleichen, in denen zweifache Suffigierung vorliegt (cf. PANOV 1968, III: 128sq.). 
manchen ist dies der vorwiegende oder gar ausschließliche Nutzungsbereich (lyži, xoduli, del'taplan, vertolet, parašjut).

Diese Vielfalt stellt der Wortbildungsanalyse beträchtliche Hindernisse in den Weg. Konkrete Substantive sind typischerweise nicht von anderen Wörtern abgeleitet; wir haben deshalb den Algorithmus, nach dem die Wortbildungsparadigmen konstruiert werden, in erster Linie für primäre Lexeme konzipiert. Bei abgeleiteten Lexemen führt das Verfahren manchmal zu unerwünschten Ergebnissen: so kann etwa vertodrom nicht in das Wortbildungsparadigma von vertolet aufgenommen werden, da vertolet nicht in ihm formal enthalten ist.

Charakteristisch für die Wortbildungsparadigmen der Substantive der Kategorie sredstva peredviženija (Fortbewegungsmittel) sind Komposita, in denen das Erstglied auf die zwei ersten Silben gekürzt wurde; im Wortbildungsparadigma von velosiped finden wir etwa

velotrenažer, veloèrgometr, velokoljaska, velorikša, velokamera, velosipedist, velosipedistka, veloturist, velofigurist, velofiguristka, velostadion, velostojanka, velotrassa, velotrek, velodrom, velobaza, velosekcija, velozavod, velogonki, velokross, veloprobeg, velobol, velotur, velosport, velostroenie.

Ähnlich verhalten sich avto... und moto... Solche Elemente können sogar kumuliert werden: motovelosport, avtomotoklub etc. Dieser Wortbildungstyp, der zwischen Komposition und Präfigierung steht und den agglutinativen Tendenzen in der Wortbildung der zeitgenössischen russischen Literatursprache entgegenkommt, wurde auch auf einheimisches Wortgut ausgedehnt (cf. das soeben zitierte vertodrom).

Die große Leichtigkeit, mit der solche Komposita gebildet werden, zeugt von der relativen Selbständigkeit des Erstgliedes; es stellt sich die Frage, ob wir es mit abgeleiteten Wörtern oder mit Syntagmen zu tun haben, die aus einem (unflektierbaren) Adjektiv und einem Substantiv bestehen. Entscheidet sich man für das letztere, so müßte man sie aus dem Wortbildungsparadigma ausschließen. Da wir Komposita von der Forderung nach Vollständigkeit der Beschreibung prinzipiell ausgenommen haben, bleibt deren Eingliederung in das Wortbildungsparadigma für den Bestand der Wortbildungskategorien ohne Folgen; wo sich jedoch das Verhältnis zwischen Komposition und Affigierung so sehr zugunsten der ersteren verschiebt, ist es nicht mehr sinnvoll, die Komposita in Wortbildungskategorien einzuordnen, die an affixalen Ableitungen entwickelt wurden.

Die größte Schwierigkeit stellt jedoch die semantische Heterogenität unserer Ausgangssubstantive dar; man betrachte dafür die substantivische Zone der Wortbildungsparadigmen der Tierbezeichnung verbljud, des Körperteils noga und eines modernen Fortbewegungsmittels, vertolet: 
VERBLJUD 'Kamel'

verbljudka 'Corispermum' (Pflanze, die u.a. den Kamelen als Nahrung dient) verbljužatnik dass. $_{1}$

verbljudki 'Raphidioptera' (Insektenordnung, bei deren Mitglieder der Hals an den Hals eines Kamels erinnert) verbljudovye 'Kamelartige' verbljudica 'Kamelweibchen' verbljužonok 'Kameljunges' verbljužatnik, 'Kameltreiber' verbljužatina 'Kamelfleisch' verbljužina 'Kamelfleisch; Kamelfell' verbljudovodstvo 'Kamelzucht'

NOGA 'Fuß'

nožen'ka 'dem. zu noga' nožonka 'dem. zu noga' nožka 'dem. zu noga' nožišča 'aug. zu noga'

nogavka 'ein um den Fuß eines Huhnes gebundenes oder genähtes Band; der Strumpf (bes. der Frauen); die Fußfetzen zu den Bastschuhen; der Strumpfschaft der Tschuwaschen- und Tscheremissenfrauen; eine oberhalb der Fußknöchel geschlungene rote Binde aus Kamelgarn'

nogovica 'Strumpfschaft, Gamasche'

pronožka 'Querleiste, Querriegel (als Verbindung der Füße eines Tisches, Stuhles etc.)'

iznože 'Fußende des Bettes; Fußschemel'

obnož, obnožka 'an den Hinterbeinen (gelben Höschen) der Bienen haftender Blütenstaub'

obnożki 'kurze Wolle von den Füßen der Schafe' Wilden)'

ponoži 'Fußketten, Fußschellen; Beinharnisch, Beinschienen; Beinspangen (der

podnožie 'alles, was zur Unterlage für die Füße dient; Fuß eines Berges, einer Säule; Piedestal, Sockel'

podnožka 'Trittbrett; Fußstoß (beim Balgen)'

podnožki 'der Trauteppich'

raznoga, raznožka 'Sperrholz, Spreizbein; Querleiste; Fußgestell, Stativ; Sägebock; Zirkel' 
VERTOLET 'Hubschrauber'

\author{
vertodrom 'Hubschrauberlandeplatz' \\ vertoletonosec 'Hubschrauberträger' \\ vertoletčik, 'Hubschraubertechniker' \\ vertoletčik, 'Pilot eines Hubschraubers' \\ vertoletčica, Fem. zu vertoletčik, \\ vertoletčica: Fem. zu vertoletčik. \\ vertoletostroitel' 'Hubschrauberbauer' \\ vertoletostroenie 'Hubschrauberbau'
}

Jedes der drei Wortbildungsparadigmen enthält Ableitungen, die für das betreffende Substantiv charakteristisch sind, aber aus pragmatischen Gründen von den übrigen beiden nicht abgeleitet werden können: im Wortbildungsparadigma von verbljud sind dies etwa verbljužonok oder verbljużatina; im Wortbildungsparadigma von noga Bezeichnungen für Kleidungsstücke und dgl., die zu diesem Körperteil in metonymischer Beziehung stehen (nogavka, nogovica, ponoži); im Wortbildungsparadigma von vertolet Ableitungen, die mit dem Bau (vertoletostroitel', vertoletostroenie) oder der militärischen Nutzung von Hubschraubern verbunden sind (vertoletonosec). Bei der Aufstellung von Wortbildungskategorien ist man vor folgende Alternative gestellt:

a) Man kann für jede dieser Ableitungen eine eigene Wortbildungskategorie mit spezifischer Bedeutung aufstellen -- in diesem Fall muß das Ökonomieprinzip verletzt werden: es wäre vergeblich, nach einem konkreten Wortbildungsparadigma zu suchen, in dem die Kategorie 'junges ...' zu der Kategorie 'Kleidungsstück, das an ... getragen wird' oder die Kategorie 'Fleisch von ...' zur Kategorie 'Konstrukteur von ...' in Opposition steht.

b) Man kann versuchen, die Ableitungen zu Wortbildungskategorien mit allgemeiner Bedeutung zusammenzufassen -- indem man etwa verbljuzatnik und vertoletčik (was noch relativ plausibel ist) oder verbljuzaatina und nogavka (aufgrund der gemeinsamen metonymischen Komponente) einer einzigen Kategorie zuordnet. Es ist offensichtlich, daß in diesem Fall das Natürlichkeitsprinzip verletzt wird.

Einen Ausweg aus diesem Dilemma gibt es nicht. Wir schließen daraus, daß die Annahme, den Wortbildungsparadigmen der Substantive der Kategorie sredstva peredviženija (Fortbewegungsmittel) liege ein gemeinsames abstraktes Wortbildungsparadigma zugrunde, fallengelassen werden muß. 


\subsection{Schlußbemerkungen}

Wir haben in dieser Arbeit die Methode der paradigmatischen Wortbildungsanalyse auf einen geschlossenen Wortschatzbereich angewandt. Ausgehend von den vier abstrakten Wortbildungsparadigmen, die wir aufgestellt haben, sind wir nun in der Lage, die in der Einleitung gestellten Fragen nach dem Allgemeinheitsgrad von Wortbildungskategorien bzw. dem Anteil der Wortbildungsbedeutung an der lexikalischen Bedeutung des abgeleiteten Wortes etwas genauer zu beantworten und unsere Antworten unter Rückgriff auf die aufgestellten Analyseprinzipien explizit zu begründen. Die Wortbildungskategorie läßt sich im Rahmen der paradigmatischen Methode von zwei Seiten her abgrenzen: sie darf höchstens all jene semantischen Komponenten enthalten, die allen Ableitungen, die unter sie fallen, gemeinsam sind; und sie muß mindestens jene semantischen Komponenten enthalten, die diese Ableitungen von ihren Nachbarableitungen im Wortbildungsparadigma unterscheiden. Mit Hilfe dieses Kriteriums läßt sich etwa sagen, daß die Bedetungskomponente 'zerstoßen' in česnočnica 'hölzerner Mörser zum Zerstoßen des Knoblauchs' oder die Bedeutungskomponenten 'Suppe' in svekol'nik bzw. 'Pastete' in kapustnik idiomatisch sind; die Bedeutungskomponenten 'Käfig, Gehege' und 'Gefäß zum Braten' in gusjatnica bzw. gusjatnja haben dagegen systematischen Charakter. Dieselbe Bedeutungskomponente kann übrigens in einigen Ableitungen zur Wortbildungsbedeutung gehören, in anderen nicht: man vergleiche in dieser Hinsicht abrikosovka, limonovka und abrikotin, limonad: im ersten Paar darf man sogar die Bedeutungskomponente 'alkoholisch' als Bestandteil der Wortbildungsbedeutung ansehen, da sie allen Ableitungen der betreffenden Wortbildungskategorie gemeinsam ist; im zweiten dagegen hat nicht nur sie, sondern auch die Bedeutungskomponente 'Getränk' idiomatischen Charakter, da die Wortbildungskategorie von abrikotin und limonad viele Substantive umfaßt, die keine Getränkebezeichnungen sind: tykvennik, višnjak etc.

Bei der Durchsicht unserer Wortbildungskategorien fällt auf, daß das Verhältnis zwischen idiomatischer Bedeutung und Wortbildungsbedeutung in ihnen recht unterschiedlich ausfallen kann: in Wortbildungskategorien wie FIL (aistenok, fazanenok etc.) oder CAR (fazanina, utjatina etc.) ist die Wortbildungsbedeutung klar umrissen, und die meisten Ableitungen weisen keine idiomatischen Bedeutungskomponenten auf; in Wortbildungskategorien wie RES (abrikotin, bananovoz etc.) oder ALI (goroxovica, kapustnik etc.) ist es genau umgekehrt: die idiomatischen Bedeutungskomponenten der einzelnen Ableitungen sind reich, die Wortbildungsbedeutung bleibt vage und unbestimmt. Bezeichnenderweise sind dies die Kategorien, die auch in formaler Hinsicht die größte Vielfalt aufweisen, während sich die Ableitungen von Kategorien, in denen die systematischen Bedeutungskomponenten gegenüber den idiomatischen überwiegen, um wenige Suffixe gruppieren. Demnach wäre nicht die Wortbildungskategorie, sondern der Wortbildungstyp als die grundlegende Einheit des Wortbildungssystems anzusehen. Dieses Ergebnis ist umso bemerkenswerter, als unsere Analyse ja gerade die entgegengesetzte Annahme einer strikten 
Trennung von Ausdruck und Inhalt im Bereich der Wortbildung zu ihrem methodischen Ausgangspunkt machte.

Eine nennenswerte Revision unserer Vorstellungen von der desubstantivischen Wortbildung hat die Anwendung der paradigmatischen Wortbildungsanalyse demnach nicht erbracht; ihre Ergebnisse fallen zum Teil sogar hinter unseren Erwartungen zurück, indem manche Wortbildungskategorien, die man kaum miteinander verschmelzen kann, mangels entsprechender Oppositionen auf der paradigmatischen Achse nicht voneinander differenziert werden können. In vielen Fällen liegt dies an der Unzulänglichkeit des Ausgangsmaterials (Korpuslücken; mangelhafte Bedeutungserklärungen in den einsprachigen Wörterbüchern des Russischen); zu einem nicht geringen Teil dürfte es aber auch darauf zurückzuführen sein, daß der Bereich der desubstantivischen Wortbildung im Vergleich mit der deverbalen oder deadjektivischen ein geringeres Ausmaß an paradigmatischer Strukturierung aufweist.

Doch sind es gerade die Schwierigkeiten, die die Anwendung der paradigmatischen Methode mit sich brachte, die zu den interessantesten Fragen Anlaß geben. Am glattesten ging die Wortbildungsanalyse in der taxinomisch-biologischen Kategorie pticy (Vögel) vonstatten; auch in den Kategorien frukty (Obst) und ovošči (Gemüse) stießen wir auf keine größeren Hindernisse. Die Aufstellung eines abstrakten Wortbildungsparadigmas für die funktional-situative Kategorie posuda (Küchengeschir und Besteck) ist recht problematisch; und in der rein funktionalen Kategorie sredstva peredviženija (Fortbewegungsmittel) waren die Forderungen der Ökonomie, der Vollständigkeit und der Natürlichkeit überhaupt nicht miteinander in Einklang zu bringen. Dieses Ergebnis läßt vermuten, daß bestimmte Kategorien konkreter Substantive in höherem Maße wortbildungsrelevant sind als andere; weitere Wortbildungsanalysen müssen zeigen, ob biologische Kategorien in dieser Hinsicht generell besser abschneiden als funktionale und ob über diese grundsätzliche Dichotomie hinaus auch andere Faktoren (z.B. die Kategorisierungsebene) eine Rolle spielen. 
Anhang 1: Produktionsnormen zu fünf Kategorien konkreter Substantive des Russischen (Exemplar-Dominanz)

Auf den folgenden Seiten wird ein Teil der Ergebnisse eines Produktionsexperimentes vorgestellt, das während eines zweimonatigen Moskauaufenthaltes im September/Oktober 1993 durchgeführt wurde.' Die Vpn waren überwiegend Studentinnen und Studenten der Russistik bzw. der Sprachwissenschaft, aber auch die Schulklasse eines humanistischen Gymnasiums sowie eine Gruppe von Mitarbeitern des Instituts für Kristallographie der Rußländischen Akademie der Wissenschaften, die sich sowohl durch ihr Alter als auch durch ihre Ausbildung von den übrigen Vpn stark abheben. In den meisten Gruppen hatten die weiblichen Versuchspersonen ein deutliches Übergewicht. Da uns die Ergebnisse hier lediglich zur Festsetzung des Mitgliederbestandes einer Kategorie sowie zu einer ersten Orientierung über die Entfernung einzelner Mitglieder von deren Zentrum dienen, fallen diese Mängel nicht so stark ins Gewicht. Die Arbeitsbedingungen in Moskau erlaubten es nicht, bei der Organisation und Durchführung des Experimentes die hohen Standards zu erreichen, die etwa MANNHAUPT 1983 einhält.

Die Versuchsdurchführung, bei der wir uns an MANNHAUPT 1983 anlehnten, fand folgendermaßen statt: es wurden Produktionsnormen zu zehn Kategorien von insgesamt 94 Vpn (56 Frauen und 38 Männern) erfaßt. Die Vpn gehörten vier verschiedenen Gruppen an, mit denen in je einer Sitzung gearbeitet wurde:

a) Die Teilnehmer einer Vorlesung am Moskovskij Gosudarstvennyj universitet -- in ihrer Mehrzahl Studierende des 3. Studienjahres der Russistik im Alter von 1824 Jahren sowie einige Personen höheren Alters (19 Frauen und 17 Männer);

b) Die Teilnehmer einer Übung an der Sprachwissenschaftlichen Fakultät des Rossijskij Gosudarstvennyj Gumanitarnyj Universitet -- 6 Studenten und 18 Studentinnen des 1. Studienjahres im Alter von 16-24 Jahren;

c) Die Schulklasse 11c des Gumanitarnyj licejj pri Rossijskom Gosudarstvennom Gumanitarnom Universitete (Schule Nr. 1276), die aus 7 Jungen und 10 Mädchen im Alter von 16-17 Jahren bestand;

d) 8 Mitarbeiter und 9 Mitarbeiterinnen des Institut Kristallografii RAN im Alter von 27-72 Jahren mit naturwissenschaftlicher Ausbildung.

Jede Vpn erhielt drei Blätter. Auf dem ersten wurden die Vpn gebeten, Alter, Beruf, Geschlecht und Bildungsgrad anzugeben. Darunter war die Versuchsinstruktion abgedruckt, deren russischer Text folgendermaßen lautet (das Original war natürlich nicht transliteriert):

' Für wertvolle Hilfe bei der Durchführung der Experimente danke ich R.M. Frumkina, A.V. MiXeEv, N.A. Tixomirova und V.M. Żivov. 
Predmet nastojaščego issledovanija -- ustanovit', $v$ kakom vide predstavleno $v$ našem soznanii značenie slov, kotorye my upotrebljaem $v$ povsednevnoj reči. $V$ častnosti, nas interesuet svjaz’ meždu slovom, oboznačajuščim celuju kategoriju predmetov, i slovami, oboznačajuščimi otdel'nye predmety ètoj kategorii.

Ja Vam budu čitat' slova i slovosočetanija, oboznačajuščie otdel'nye kategorii predmetov; ja prošu Vas posle ètogo zapisat' kak možno bol'še slov, vxodjaščix v dennuju kategoriju. Naprimer, esli ja Vam pročitaju slovo mebel', Vy možete napisat' $\mathrm{v}$ otvet slova stol, stul, škaf, bufet i td.; esli ja Vam pročitaju slovosočetanie naselennyj punkt, Vy možete napisat' $v$ otvet slova selo, gorod, derevrija i td. Ja pročtu každoe slovo ili slovosočetanie dva raza; potom u Vas budet 30 sekund, čtoby zapisat' Vaši otvety. V moem spiske vsego 10 kategorij; pered Vami dva razlinovannyx lista dlja Vašix otvetov. Prošu Vas zapisat' slova, vxodjaščie $v$ pervuju kategoriju, pod nomerom 1 ; slova, vxodjaščie vo vtoruju kategoriju, pod nomerom 2; i td.

Očen’ važno, čtoby Vy zapisyvali slova $v$ tom porjadke, $v$ kotorom oni Vam prixodjat $v$ golovu; prošu $V$ as rabotat' sosredotočenno i ne preryvat' èksperiment voprosami ili zamečanijami (posle èksperimenta ja gotov otvetit' na Vaši voprosy); esli Vam kakaja-nibud' kategorija neizvestna, to ostav'te ee mesto pustym i ždite, poka ja pročtu slovo, sootvetstvujuščee sledujuščej kategorii.

Die zwei folgenden Blätter waren liniert und im Abstand von fünf Zeilen durchnumeriert. Die Numerierung (1-10) zeigte an, an welchen Stellen die Kategoriennamen einzutragen waren.

Nachdem die Blätter verteilt waren, wurde die Instruktion vorgelesen. Daraufhin fand die eigentliche Erhebung statt, die folgende Kategorien umfaßte:

Odežda
Frukty
Muzykal'nye instrumenty
Sladosti
Pticy
Obuv'
Ovošči
Sredstva peredviženija
Napitki
Posuda

Da am Anfang der Experimentreihe nicht abzusehen war, wie viele Gruppen insgesamt zur Verfügung stehen würden, wurde diese Reihenfolge in den einzelnen 
Experimenten nicht variiert. Um die Positionseffekte etwas einzuschränken, wurden versucht, eng verwandte Kategorien (odežda - obuv', frukty - ovošči) in möglichst großer Entfernung voneinander anzubieten. Posuda wurde wegen seiner semantischen Eigenart, die Rückfragen provozieren konnte, an das Ende gesetzt.

Da die Räume, in denen das Experiment durchgeführt wurde, teilweise recht groß waren, wurde jeder Kategorienname zweimal hintereinander vorgelesen. Daraufhin erhielten die Vpn 30 Sekunden Zeit, um ihre Antworten aufzuschreiben. Jede Sitzung dauerte ca. 15 Minuten.

Bei der Auswertung der Ergebnisse wurde folgendermaßen vorgegangen: zunächst wurden die Teile der Fragebögen, die sich auf die hier untersuchten Kategorien bezogen, mit einem Textverarbeitungsprogramm in den Rechner eingegeben. Dabei wurden abweichende orthographische Fassungen, sofern sie sich nicht (serviz/servis, snegir'/snigir') oder kaum (trollejbus/trolejbus, patissony/patisony) in der Lautung unterschieden, nach den Normen der russischen Rechtschreibung vereinheitlicht und gelegentlich vorkommende Abkürzungen (trol. für trollejbus, tram. für tramvaj) aufgelöst. Auch die Zeichensetzung wurde vereinheitlicht: alle Kategorienmitglieder wurden voneinander durch Kommata getrennt (in den Fragebögen selber sind oft nur Leerzeichen vorhanden). Bei der anschließenden Zählung wurden Singular- und Pluralformen zusammengefaßt. Deminutiva (vaza/vazočka) und lexikalische Varianten (žuravel'žzuravl', grif/grifon) wurden jedoch getrennt ausgezählt.

Zur Schätzung der Zuverlässigkeit wurde (nach dem Vorbild von MANNHAUPT 1983) die Gesamtstichprobe in zwei Teilstichproben von je $47 \mathrm{Vpn}$ geteilt, in denen die einzelnen Teilgruppen a) bis d) gleich stark vertreten waren. Anschließend wurden die beiden Teilstichproben getrennt ausgewertet und für jede Kategorie der Korrelationskoeffizient zwischen den Häufikeiten der einzelnen Mitglieder errechnet. Dabei ergaben sich folgende Koeffizienten:

$\begin{array}{ll}\text { pticy } & 0,92 \\ \text { frukty } & 0,94 \\ \text { ovošči } & 0,97 \\ \text { posuda } & 0,99 \\ \text { sredstva perevidženija } & 0,96\end{array}$

Läßt man bei dieser Berechnung jene Kategorienmitglieder aus, die in jeder der beiden Gruppen höchstens zweimal genannt wurden (d.h. ermittelt man die Korrelationskoeffizienten nur über jene Kategorienmitglieder, die in mindestens einer Teilstichprobe mit einer Häufigkeit von drei oder mehr auftraten), so erhält man folgende Korrelationskoeffizienten: 
pticy

frukty

0,90

ovošči

posuda

sredstva peredviženija

Bei der nun folgenden Übersicht über die Ergebnisse ist folgendes zu beachten: die Buchstaben A, B, C, D entsprechen den oben aufgezählten Versuchsgruppen; unter dem Großbuchstaben ist die Anzahl der Gesamtnennungen, unter dem Kleinbuchstaben die der Erstnennungen des jeweiligen Kategorienmitgliedes aufgeführt. Die Kategorienmitglieder sind nach der absteigenden Anzahl der Gesamtnennungen geordnet. Aus technischen Gründen sind die Kategorienmitglieder, die gleich oft genannt wurden, ebenfalls nach absteigender alphabetischer Reihenfolge (des deutschen Alphabets) aufgeführt. Alle Wörter stehen im Nominativ Singular -- auch solche, die in den Fragebögen nur im Plural vorkamen, wie etwa ovošči oder nogi. Der Nominativ Plural steht nur dort, wo eine Singularform mit derselben Bedeutung nicht existiert.

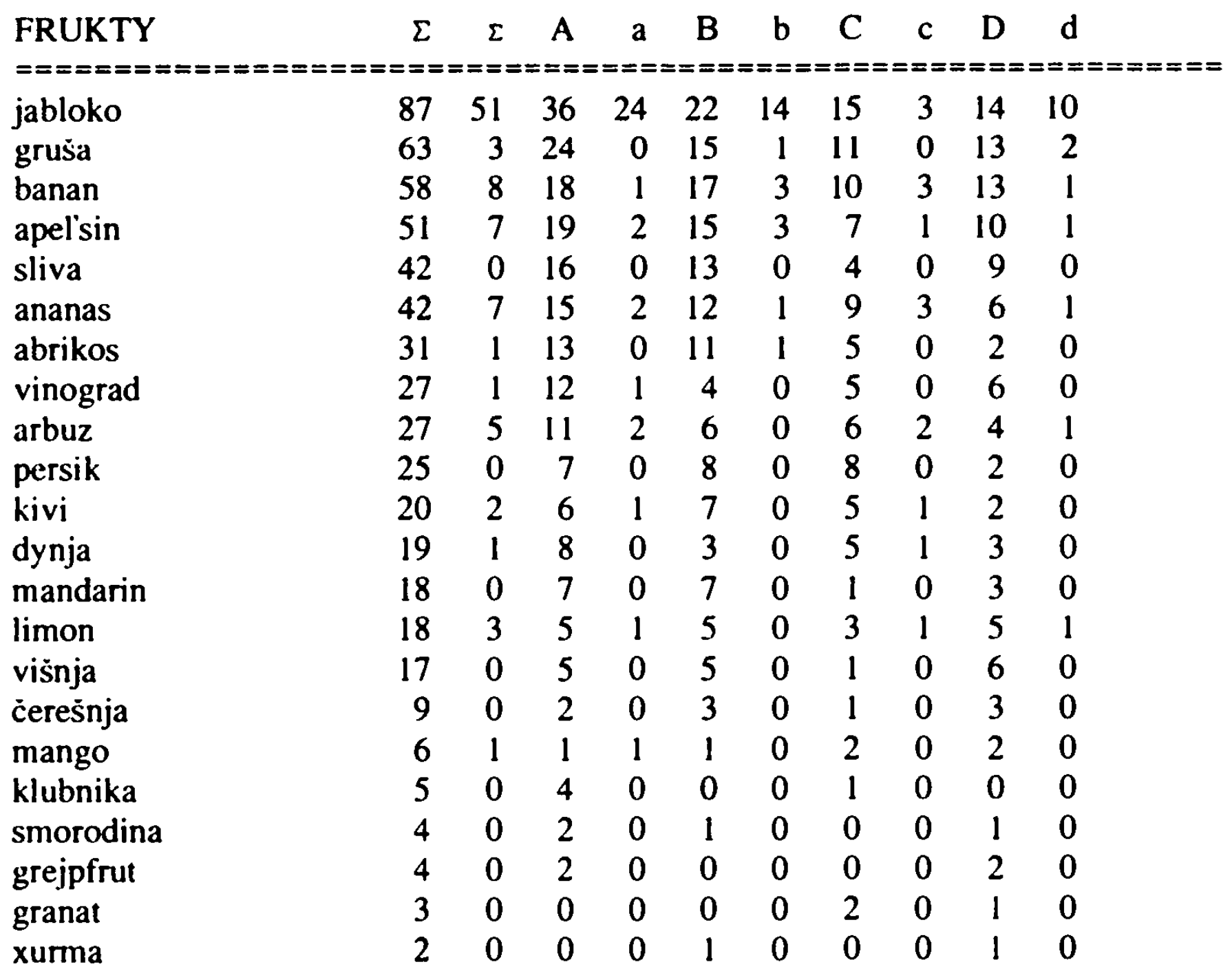




$\begin{array}{lllllllllll}\text { rjabina } & 2 & 0 & 0 & 0 & 1 & 0 & 0 & 0 & 1 & 0 \\ \text { malina } & 2 & 1 & 1 & 1 & 0 & 0 & 1 & 0 & 0 & 0 \\ \text { kryżovnik } & 2 & 0 & 1 & 0 & 0 & 0 & 0 & 0 & 1 & 0 \\ \text { fejxua } & 2 & 0 & 0 & 0 & 0 & 0 & 2 & 0 & 0 & 0 \\ \text { ajva } & 2 & 0 & 0 & 0 & 1 & 0 & 0 & 0 & 1 & 0 \\ \text { zemljanika } & 1 & 0 & 0 & 0 & 0 & 0 & 1 & 0 & 0 & 0 \\ \text { tykva } & 1 & 1 & 0 & 0 & 0 & 0 & 1 & 1 & 0 & 0 \\ \text { tern } & 1 & 0 & 0 & 0 & 0 & 0 & 0 & 0 & 1 & 0 \\ \text { ovošč } & 1 & 1 & 0 & 0 & 1 & 1 & 0 & 0 & 0 & 0 \\ \text { kokos } & 1 & 0 & 0 & 0 & 0 & 0 & 1 & 0 & 0 & 0 \\ \text { inžir } & 1 & 0 & 1 & 0 & 0 & 0 & 0 & 0 & 0 & 0 \\ \text { frukt } & 1 & 1 & 0 & 0 & 0 & 0 & 1 & 1 & 0 & 0 \\ \text { eževika } & 1 & 0 & 0 & 0 & 0 & 0 & 1 & 0 & 0 & 0 \\ \text { avokado } & 1 & 0 & 0 & 0 & 0 & 0 & 1 & 0 & 0 & 0\end{array}$

tokens

$\begin{array}{llllllllll}597 & 94 & 216 & 36 & 159 & 24 & 110 & 17 & 112 & 17\end{array}$

types

$\begin{array}{llllllllll}36 & 16 & 23 & 10 & 22 & 7 & 27 & 10 & 25 & 7\end{array}$

$\begin{array}{lllllllllll}\text { PTICY } & \Sigma & \Sigma & \mathrm{A} & \mathrm{a} & \mathrm{B} & \mathrm{b} & \mathrm{C} & \mathrm{c} & \mathrm{D} & \mathrm{d}\end{array}$

$\begin{array}{lrrrrrrrrrr}\text { vorobej } & 64 & 10 & 26 & 3 & 18 & 2 & 7 & 0 & 13 & 5 \\ \text { vorona }^{2} & 62 & 7 & 21 & 2 & 17 & 2 & 11 & 2 & 13 & 1 \\ \text { golub }_{\text {orel }} & 39 & 6 & 13 & 2 & 12 & 4 & 7 & 0 & 7 & 0 \\ \text { sinica } & 37 & 10 & 18 & 7 & 7 & 2 & 5 & 0 & 7 & 1 \\ \text { popugaj } & 33 & 2 & 12 & 2 & 7 & 0 & 5 & 0 & 9 & 0 \\ \text { utka } & 28 & 11 & 9 & 6 & 8 & 0 & 7 & 5 & 4 & 0 \\ \text { solovej } & 19 & 4 & 7 & 0 & 6 & 3 & 1 & 0 & 5 & 1 \\ \text { kurica } & 19 & 3 & 7 & 2 & 3 & 0 & 5 & 0 & 4 & 1 \\ \text { soroka } & 19 & 2 & 8 & 2 & 8 & 0 & 1 & 0 & 2 & 0 \\ \text { jastreb } & 17 & 0 & 9 & 0 & 2 & 0 & 3 & 0 & 3 & 0 \\ \text { galka } & 17 & 2 & 7 & 0 & 4 & 1 & 4 & 1 & 2 & 0 \\ \text { djatel } & 17 & 1 & 2 & 1 & 7 & 0 & 4 & 0 & 4 & 0 \\ \text { snegir' } & 15 & 2 & 7 & 1 & 3 & 0 & 3 & 1 & 2 & 0 \\ \text { kanarejka } & 13 & 1 & 6 & 0 & 3 & 0 & 2 & 0 & 2 & 1 \\ \text { żuravl' } & 13 & 1 & 5 & 1 & 3 & 0 & 2 & 0 & 3 & 0 \\ \text { straus } & 12 & 0 & 4 & 0 & 3 & 0 & 2 & 0 & 3 & 0 \\ \text { sokol } & 12 & 0 & 6 & 0 & 4 & 0 & 2 & 0 & 0 & 0 \\ \text { lebed' } & 12 & 2 & 4 & 0 & 4 & 2 & 2 & 0 & 2 & 0 \\ \text { gus' } & 12 & 3 & 3 & 0 & 2 & 0 & 2 & 1 & 5 & 2 \\ & 11 & 2 & 3 & 0 & 4 & 0 & 1 & 1 & 3 & 1\end{array}$

${ }^{2}$ Nebst der Pluralform vorony, die man auch zu voron stellen kann. 
grač

lastočka

drozd

pavlin

ivolga

voron

žavoronok

skvorec

grif

čajka

aist

zjablik

sojka

ščegol

indjuk

fazan

sova

petux

kukuška

koršun

berkut

pingvin

perepel

pelikan

kolibri

gluxar

drofa

al'batros

żuravel'

zimorodok

trjasoguzka

teterev

striż

perepelka

malinovka

kuropatka

indjuška

caplja

zarjanka

vyp'

udod

syc̈

sviristel $\begin{array}{llllllllll}11 & 2 & 1 & 0 & 3 & 1 & 3 & 0 & 4 & 1 \\ 10 & 4 & 5 & 1 & 3 & 2 & 0 & 0 & 2 & 1\end{array}$

$\begin{array}{llllllllll}10 & 3 & 3 & 0 & 2 & 0 & 4 & 2 & 1 & 1\end{array}$

$\begin{array}{llllllllll}9 & 1 & 6 & 1 & 2 & 0 & 0 & 0 & 1 & 0\end{array}$

$\begin{array}{llllllllll}8 & 2 & 4 & 1 & 2 & 1 & 2 & 0 & 0 & 0\end{array}$

$\begin{array}{llllllllll}7 & 1 & 3 & 1 & 4 & 0 & 0 & 0 & 0 & 0\end{array}$

$\begin{array}{llllllllll}6 & 1 & 3 & 0 & 0 & 0 & 2 & 0 & 1 & 1\end{array}$

$\begin{array}{llllllllll}6 & 1 & 0 & 0 & 3 & 1 & 1 & 0 & 2 & 0\end{array}$

$\begin{array}{llllllllll}6 & 0 & 4 & 0 & 0 & 0 & 1 & 0 & 1 & 0\end{array}$

$\begin{array}{llllllllll}6 & 1 & 3 & 0 & 2 & 1 & 1 & 0 & 0 & 0\end{array}$

$\begin{array}{llllllllll}6 & 1 & 1 & 0 & 2 & 1 & 3 & 0 & 0 & 0\end{array}$

$\begin{array}{llllllllll}5 & 1 & 1 & 0 & 1 & 0 & 2 & 1 & 1 & 0\end{array}$

$\begin{array}{llllllllll}5 & 0 & 3 & 0 & 1 & 0 & 0 & 0 & 1 & 0\end{array}$

$\begin{array}{llllllllll}5 & 1 & 0 & 0 & 1 & 1 & 2 & 0 & 2 & 0\end{array}$

$\begin{array}{llllllllll}5 & 0 & 2 & 0 & 3 & 0 & 0 & 0 & 0 & 0\end{array}$

$\begin{array}{llllllllll}5 & 0 & 3 & 0 & 1 & 0 & 1 & 0 & 0 & 0\end{array}$

$\begin{array}{llllllllll}4 & 0 & 0 & 0 & 3 & 0 & 0 & 0 & 1 & 0\end{array}$

$\begin{array}{llllllllll}4 & 0 & 3 & 0 & 1 & 0 & 0 & 0 & 0 & 0\end{array}$

$\begin{array}{llllllllll}4 & 0 & 2 & 0 & 1 & 0 & 0 & 0 & 1 & 0\end{array}$

$\begin{array}{llllllllll}4 & 0 & 2 & 0 & 1 & 0 & 0 & 0 & 1 & 0\end{array}$

$\begin{array}{llllllllll}4 & 0 & 2 & 0 & 0 & 0 & 1 & 0 & 1 & 0\end{array}$

$\begin{array}{llllllllll}3 & 0 & 0 & 0 & 1 & 0 & 2 & 0 & 0 & 0\end{array}$

$\begin{array}{llllllllll}3 & 0 & 0 & 0 & 3 & 0 & 0 & 0 & 0 & 0\end{array}$

$\begin{array}{llllllllll}3 & 0 & 2 & 0 & 0 & 0 & 1 & 0 & 0 & 0\end{array}$

$\begin{array}{llllllllll}3 & 0 & 1 & 0 & 1 & 0 & 1 & 0 & 0 & 0\end{array}$

$\begin{array}{llllllllll}3 & 0 & 1 & 0 & 1 & 0 & 0 & 0 & 1 & 0\end{array}$

$\begin{array}{llllllllll}3 & 0 & 2 & 0 & 1 & 0 & 0 & 0 & 0 & 0\end{array}$

$\begin{array}{llllllllll}3 & 0 & 2 & 0 & 0 & 0 & 1 & 0 & 0 & 0\end{array}$

$\begin{array}{llllllllll}2 & 0 & 0 & 0 & 1 & 0 & 1 & 0 & 0 & 0\end{array}$

$\begin{array}{llllllllll}2 & 1 & 1 & 1 & 0 & 0 & 1 & 0 & 0 & 0\end{array}$

$\begin{array}{llllllllll}2 & 0 & 0 & 0 & 1 & 0 & 1 & 0 & 0 & 0\end{array}$

$\begin{array}{llllllllll}2 & 0 & 1 & 0 & 0 & 0 & 0 & 0 & 1 & 0\end{array}$

$\begin{array}{llllllllll}2 & 0 & 1 & 0 & 1 & 0 & 0 & 0 & 0 & 0\end{array}$

$\begin{array}{llllllllll}2 & 0 & 0 & 0 & 2 & 0 & 0 & 0 & 0 & 0\end{array}$

$\begin{array}{llllllllll}2 & 0 & 1 & 0 & 0 & 0 & 0 & 0 & 1 & 0\end{array}$

$\begin{array}{llllllllll}2 & 1 & 0 & 0 & 1 & 0 & 1 & 1 & 0 & 0\end{array}$

$\begin{array}{llllllllll}2 & 0 & 1 & 0 & 1 & 0 & 0 & 0 & 0 & 0\end{array}$

$\begin{array}{llllllllll}2 & 1 & 2 & 1 & 0 & 0 & 0 & 0 & 0 & 0\end{array}$

$\begin{array}{llllllllll}1 & 0 & 0 & 0 & 1 & 0 & 0 & 0 & 0 & 0\end{array}$

$\begin{array}{llllllllll}1 & 0 & 0 & 0 & 0 & 0 & 0 & 0 & 1 & 0\end{array}$

$\begin{array}{llllllllll}1 & 0 & 1 & 0 & 0 & 0 & 0 & 0 & 0 & 0\end{array}$

$\begin{array}{llllllllll}1 & 0 & 0 & 0 & 1 & 0 & 0 & 0 & 0 & 0\end{array}$

$\begin{array}{llllllllll}1 & 0 & 0 & 0 & 0 & 0 & 0 & 0 & 1 & 0\end{array}$ 


\begin{tabular}{|c|c|c|c|c|c|c|c|c|c|c|}
\hline strekač & 1 & 0 & 0 & 0 & 0 & 0 & 1 & 0 & 0 & 0 \\
\hline stervjatnik & 1 & 0 & 1 & 0 & 0 & 0 & 0 & 0 & 0 & 0 \\
\hline slavka & 1 & 1 & 1 & 1 & 0 & 0 & 0 & 0 & 0 & 0 \\
\hline sinička & 1 & 0 & 0 & 0 & 1 & 0 & 0 & 0 & 0 & 0 \\
\hline ptica-sekretar' & 1 & 0 & 1 & 0 & 0 & 0 & 0 & 0 & 0 & 0 \\
\hline rjabčik & 1 & 0 & 1 & 0 & 0 & 0 & 0 & 0 & 0 & 0 \\
\hline volnistyj popugajčik & 1 & 1 & 0 & 0 & 0 & 0 & 1 & 1 & 0 & 0 \\
\hline penočka & 1 & 0 & 1 & 0 & 0 & 0 & 0 & 0 & 0 & 0 \\
\hline penka & 1 & 0 & 1 & 0 & 0 & 0 & 0 & 0 & 0 & 0 \\
\hline kozodoj & 1 & 0 & 1 & 0 & 0 & 0 & 0 & 0 & 0 & 0 \\
\hline klest & 1 & 1 & 0 & 0 & 0 & 0 & 1 & 1 & 0 & 0 \\
\hline kivi & 1 & 0 & 1 & 0 & 0 & 0 & 0 & 0 & 0 & 0 \\
\hline kakadu & 1 & 0 & 0 & 0 & 1 & 0 & 0 & 0 & 0 & 0 \\
\hline grifon & 1 & 0 & 1 & 0 & 0 & 0 & 0 & 0 & 0 & 0 \\
\hline flamingo & 1 & 0 & 1 & 0 & 0 & 0 & 0 & 0 & 0 & 0 \\
\hline cyplenok & 1 & 0 & 0 & 0 & 1 & 0 & 0 & 0 & 0 & 0 \\
\hline čibis & 1 & 0 & 0 & 0 & 0 & 0 & 0 & 0 & 1 & 0 \\
\hline čiž & 1 & 0 & 1 & 0 & 0 & 0 & 0 & 0 & 0 & 0 \\
\hline cesarka & 1 & 0 & 1 & 0 & 0 & 0 & 0 & 0 & 0 & 0 \\
\hline tokens & 666 & 94 & 256 & 36 & 180 & 24 & 111 & 17 & 119 & 17 \\
\hline types & 82 & 35 & 62 & 18 & 54 & 14 & 43 & 11 & 39 & 12 \\
\hline OVOŠĊI & $\Sigma$ & $\Sigma$ & A & $\mathbf{a}$ & B & b & $\mathrm{C}$ & c & $\mathrm{D}$ & d \\
\hline$======$ & $====$ & $===$ & $===$ & $===$ & $====$ & $====$ & $===$ & $===$ & $====$ & $===$ \\
\hline kapusta & 66 & 16 & 27 & 6 & 18 & 5 & 9 & 3 & 12 & 2 \\
\hline pomidor & 59 & 15 & 25 & 7 & 16 & 4 & 11 & 3 & 7 & 1 \\
\hline ogurec & 58 & 15 & 22 & 6 & 16 & 5 & 12 & 3 & 8 & 1 \\
\hline morkov' & 58 & 9 & 23 & 2 & 17 & 4 & 7 & 1 & 11 & 2 \\
\hline svekla & 46 & 4 & 19 & 0 & 13 & 2 & 5 & 0 & 9 & 2 \\
\hline kartofel' & 42 & 11 & 21 & 6 & 4 & 0 & 8 & 1 & 9 & 4 \\
\hline repa & 27 & 1 & 9 & 1 & 6 & 0 & 4 & 0 & 8 & 0 \\
\hline kartoška & 24 & 11 & 8 & 5 & 9 & 3 & 2 & 0 & 5 & 3 \\
\hline baklažan & 22 & 1 & 9 & 0 & 5 & 0 & 7 & 1 & 1 & 0 \\
\hline kabačok & 21 & 1 & 5 & 0 & 9 & 0 & 5 & 1 & 2 & 0 \\
\hline luk & 19 & 2 & 6 & 0 & 4 & 0 & 2 & 1 & 7 & 1 \\
\hline tykva & 16 & 2 & 7 & 0 & 5 & 1 & 1 & 1 & 3 & 0 \\
\hline perec & 15 & 1 & 4 & 0 & 3 & 0 & 6 & 0 & 2 & 1 \\
\hline redis & 11 & 0 & 5 & 0 & 3 & 0 & 2 & 0 & 1 & 0 \\
\hline rediska & 8 & 0 & 1 & 0 & 4 & 0 & 1 & 0 & 2 & 0 \\
\hline red'ka & 8 & 0 & 2 & 0 & 2 & 0 & 0 & 0 & 4 & 0 \\
\hline gorox & 7 & 0 & 1 & 0 & 3 & 0 & 2 & 0 & 1 & 0 \\
\hline
\end{tabular}




$\begin{array}{lllllllllll}\text { salat } & 6 & 0 & 4 & 0 & 0 & 0 & 0 & 0 & 2 & 0 \\ \text { patisson } & 6 & 0 & 2 & 0 & 3 & 0 & 1 & 0 & 0 & 0 \\ \text { česnok } & 6 & 0 & 2 & 0 & 1 & 0 & 0 & 0 & 3 & 0 \\ \text { brjukva } & 6 & 0 & 3 & 0 & 1 & 0 & 0 & 0 & 2 & 0 \\ \text { morkovka } & 4 & 2 & 2 & 2 & 0 & 0 & 2 & 0 & 0 & 0 \\ \text { fasol' } & 4 & 0 & 1 & 0 & 0 & 0 & 1 & 0 & 2 & 0 \\ \text { zelenyj gorošek } & 2 & 0 & 0 & 0 & 0 & 0 & 2 & 0 & 0 & 0 \\ \text { tomat } & 3 & 0 & 0 & 0 & 1 & 0 & 1 & 0 & 1 & 0 \\ \text { petruška } & 3 & 0 & 1 & 0 & 0 & 0 & 0 & 0 & 2 & 0 \\ \text { xren } & 2 & 0 & 2 & 0 & 0 & 0 & 0 & 0 & 0 & 0 \\ \text { ukrop } & 2 & 0 & 2 & 0 & 0 & 0 & 0 & 0 & 0 & 0 \\ \text { arbuz } & 2 & 2 & 1 & 1 & 0 & 0 & 1 & 1 & 0 & 0 \\ \text { zelen' } & 1 & 0 & 1 & 0 & 0 & 0 & 0 & 0 & 0 & 0 \\ \text { topinambur } & 1 & 0 & 0 & 0 & 0 & 0 & 1 & 0 & 0 & 0 \\ \text { spinat } & 1 & 0 & 0 & 0 & 1 & 0 & 0 & 0 & 0 & 0 \\ \text { repka } & 1 & 0 & 1 & 0 & 0 & 0 & 0 & 0 & 0 & 0 \\ \text { pasternak } & 1 & 0 & 0 & 0 & 0 & 0 & 0 & 0 & 1 & 0 \\ \text { luk repčatyj } & 1 & 0 & 1 & 0 & 0 & 0 & 0 & 0 & 0 & 0 \\ \text { kapusta kvašenaja } & 1 & 0 & 0 & 0 & 1 & 0 & 0 & 0 & 0 & 0 \\ \text { gorošek } & 1 & 0 & 1 & 0 & 0 & 0 & 0 & 0 & 0 & 0 \\ \text { dzukini } & 1 & 0 & 1 & 0 & 0 & 0 & 0 & 0 & 0 & 0 \\ \text { cvetnaja kapusta } & 1 & 0 & 0 & 0 & 1 & 0 & 0 & 0 & 0 & 0 \\ \text { burak } & 1 & 0 & 0 & 0 & 1 & 0 & 0 & 0 & 0 & 0 \\ \text { boby } & 1 & 0 & 0 & 0 & 0 & 0 & 0 & 0 & 1 & 0 \\ \text { artišok } & 1 & 1 & 0 & 0 & 0 & 0 & 1 & 1 & 0 & 0\end{array}$

tokens

$\begin{array}{llllllllll}566 & 94 & 219 & 36 & 147 & 24 & 94 & 17 & 106 & 17\end{array}$

types

$\begin{array}{llllllllll}42 & 16 & 32 & 9 & 25 & 7 & 24 & 11 & 25 & 9\end{array}$

SREDSTVA

$\begin{array}{lllllllllll}\text { PEREDVIŻENIJA } & \Sigma & \Sigma & \mathrm{A} & \mathrm{a} & \mathrm{B} & \mathrm{b} & \mathrm{C} & \mathrm{c} & \mathrm{D} & \mathrm{d}\end{array}$

avtobus

samolet

poezd

avtomobil'

velosiped

trollejbus

mašina

metro

tramvaj $\begin{array}{lll}66 & 13 & 25\end{array}$

$\begin{array}{llllllllll}63 & 6 & 25 & 3 & 17 & 1 & 12 & 2 & 9 & 0\end{array}$

$\begin{array}{llllllllll}50 & 2 & 17 & 0 & 13 & 1 & 10 & 0 & 10 & 1\end{array}$

$\begin{array}{llllllllll}42 & 27 & 14 & 7 & 8 & 6 & 9 & 6 & 11 & 8\end{array}$

$\begin{array}{llllllllll}41 & 6 & 16 & 3 & 11 & 0 & 8 & 1 & 6 & 2\end{array}$

$\begin{array}{llllllllll}40 & 4 & 15 & 2 & 11 & 1 & 5 & 1 & 9 & 0\end{array}$

$\begin{array}{llllllllll}39 & 18 & 18 & 9 & 8 & 5 & 8 & 2 & 5 & 2\end{array}$

$\begin{array}{llllllllll}38 & 4 & 16 & 3 & 12 & 0 & 6 & 1 & 4 & 0\end{array}$

$\begin{array}{llllllllll}35 & 2 & 13 & 0 & 10 & 0 & 5 & 0 & 7 & 2\end{array}$ 


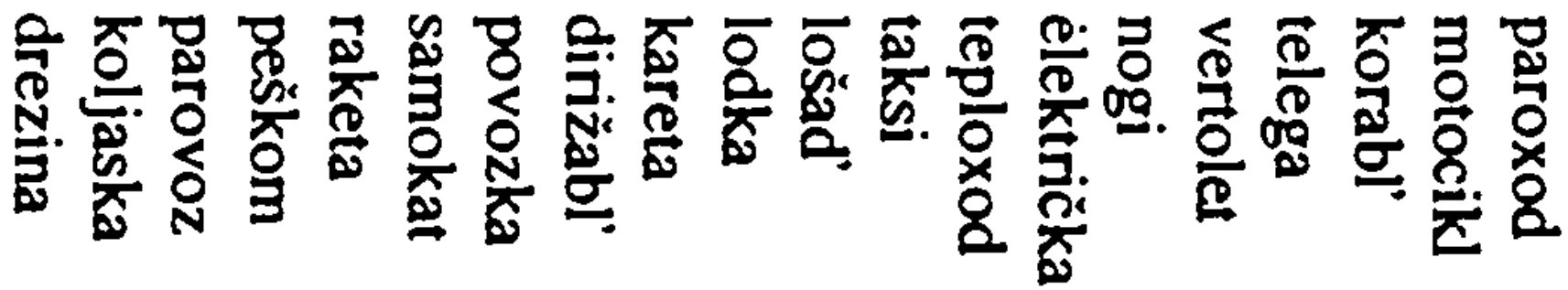

w w w w w OOO-OO-OOOTNOONOROMTO $-\sim N-1-0-A N-\omega A N A u+u a a$ $000-0000000-00-00000$ - - ON-OWw-NA-O UnN-A a nw $000000-0000-0000-0-0$ $0000-N 0000-0-0-M-M-$

$0000000000-000-00000$ - 00000000000000000000 


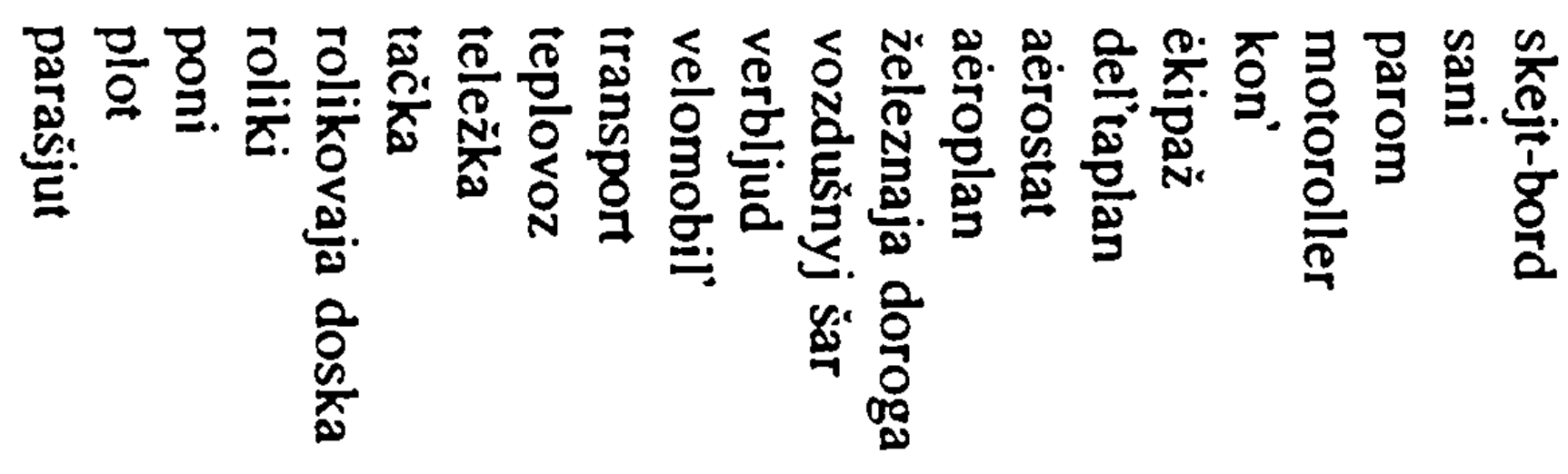

$$
\begin{aligned}
& 0000000-00000000000-0 \\
& -000--10-0000--1-0 n 00- \\
& 00000000-0000000000000 \\
& 000000000--10-0-000000 \\
& 0000000000000000000000 \\
& 000-000-0000-000-00-0- \\
& 0000000000000000000000 \\
& 0-100000000000-00 n 0-n 0 \\
& 00000000000000000000-0
\end{aligned}
$$


osel

moped

lyži

mašina (legkovaja)

lajner

kon'ki

kater

kanatnaja doroga

gorodskoj transport

funikuler

èlektropoezd

èkspress

džip

dvukolka

$\begin{array}{llllllllll}1 & 0 & 0 & 0 & 0 & 0 & 0 & 0 & 1 & 0 \\ 1 & 0 & 1 & 0 & 0 & 0 & 0 & 0 & 0 & 0 \\ 1 & 0 & 0 & 0 & 0 & 0 & 0 & 0 & 1 & 0 \\ 1 & 1 & 0 & 0 & 1 & 1 & 0 & 0 & 0 & 0 \\ 1 & 0 & 1 & 0 & 0 & 0 & 0 & 0 & 0 & 0 \\ 1 & 0 & 0 & 0 & 1 & 0 & 0 & 0 & 0 & 0 \\ 1 & 0 & 1 & 0 & 0 & 0 & 0 & 0 & 0 & 0 \\ 1 & 0 & 1 & 0 & 0 & 0 & 0 & 0 & 0 & 0 \\ 1 & 0 & 1 & 0 & 0 & 0 & 0 & 0 & 0 & 0 \\ 1 & 0 & 1 & 0 & 0 & 0 & 0 & 0 & 0 & 0 \\ 1 & 0 & 0 & 0 & 0 & 0 & 1 & 0 & 0 & 0 \\ 1 & 0 & 0 & 0 & 1 & 0 & 0 & 0 & 0 & 0 \\ 1 & 0 & 0 & 0 & 1 & 0 & 0 & 0 & 0 & 0 \\ 1 & 0 & 1 & 0 & 0 & 0 & 0 & 0 & 0 & 0\end{array}$

tokens

$\begin{array}{llllllllll}592 & 94 & 232 & 36 & 162 & 24 & 94 & 17 & 104 & 17\end{array}$

types

$\begin{array}{llllllllll}65 & 19 & 46 & 11 & 35 & 11 & 26 & 9 & 31 & 7\end{array}$

POSUDA

$\begin{array}{llllllllll}\Sigma & \Sigma & \mathrm{A} & \mathrm{a} & \mathrm{B} & \mathrm{b} & \mathrm{C} & \mathrm{C} & \mathrm{D} & \mathrm{d}\end{array}$

\begin{tabular}{|c|c|c|c|c|c|c|c|c|c|c|}
\hline tarelka & 92 & 49 & 34 & 16 & 24 & 16 & 17 & 10 & 17 & 7 \\
\hline$\check{c} a s ̌ k a^{3}$ & 78 & 16 & 28 & 7 & 19 & 3 & 15 & 2 & 16 & 4 \\
\hline bljudce & 52 & 1 & 16 & 0 & 15 & 0 & 12 & 0 & 9 & 1 \\
\hline kastrjulja & 51 & 12 & 17 & 7 & 15 & 2 & 8 & 1 & 11 & 2 \\
\hline ložka & 45 & 0 & 20 & 0 & 13 & 0 & 6 & 0 & 6 & 0 \\
\hline vilka & 39 & 0 & 16 & 0 & 11 & 0 & 7 & 0 & 5 & 0 \\
\hline čajnik & 29 & 3 & 13 & 2 & 7 & 0 & 6 & 1 & 3 & 0 \\
\hline noż & 24 & 0 & 11 & 0 & 7 & 0 & 3 & 0 & 3 & 0 \\
\hline stakan & 20 & 3 & 13 & 2 & 2 & 0 & 1 & 0 & 4 & 1 \\
\hline miska & 19 & 2 & 7 & 1 & 9 & 1 & 1 & 0 & 2 & 0 \\
\hline kružka & 14 & 1 & 8 & 0 & 5 & 1 & 0 & 0 & 1 & 0 \\
\hline skovoroda & 13 & 0 & 3 & 0 & 9 & 0 & 1 & 0 & 0 & 0 \\
\hline bljudo & 13 & 2 & 6 & 1 & 2 & 0 & 4 & 1 & 1 & 0 \\
\hline skovorodka & 11 & 0 & 3 & 0 & 3 & 0 & 2 & 0 & 3 & 0 \\
\hline kofejnik & 10 & 0 & 4 & 0 & 4 & 0 & 1 & 0 & 1 & 0 \\
\hline supnica & 9 & 1 & 2 & 0 & 4 & 0 & 1 & 1 & 2 & 0 \\
\hline saxarnica & 5 & 0 & 2 & 0 & 2 & 0 & 0 & 0 & 1 & 0 \\
\hline
\end{tabular}

${ }^{3}$ Die hohe Häufigkeit von čaška in dieser Kategorie ist dadurch zu erklären, daß der vorangehende Stimulus die Kategorie napitki war. Cf. die Ergebnisse von V.N. MASKADYNJA, in denen čaška erst an fünfter Stelle vorkommt. 


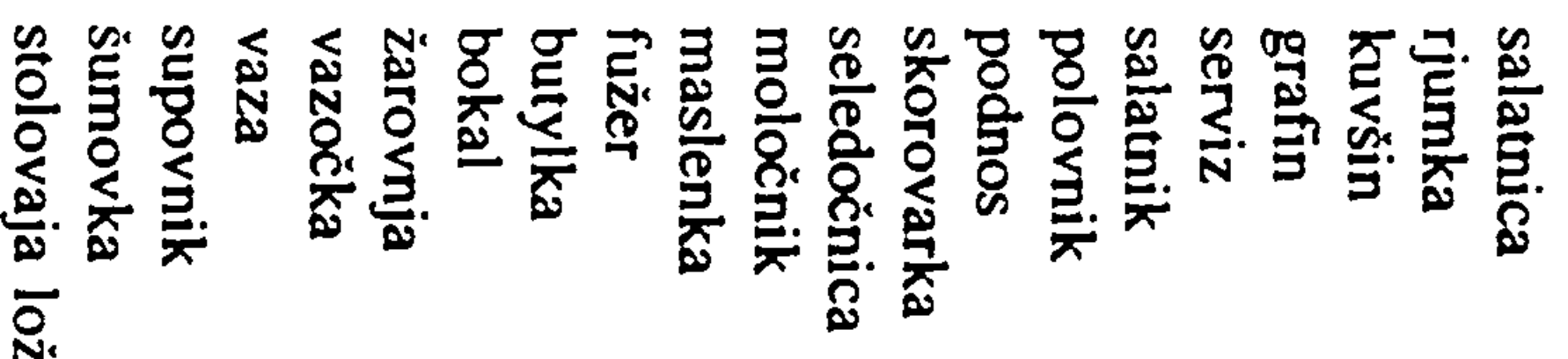

- - - - NNNNNNNWWWWA UU

$0000000000000000 N 0000$

-ORO-ORONORTONR-TNNWW

000000000000000000000

$0-000000010-1-n-10000$

$0000000000000000-0000$

$00000000000000-0-0-N N$

$0000000000000000-0000$

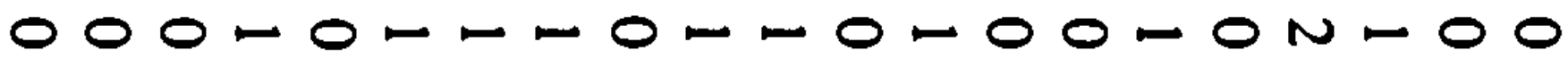

000000000000000000000 


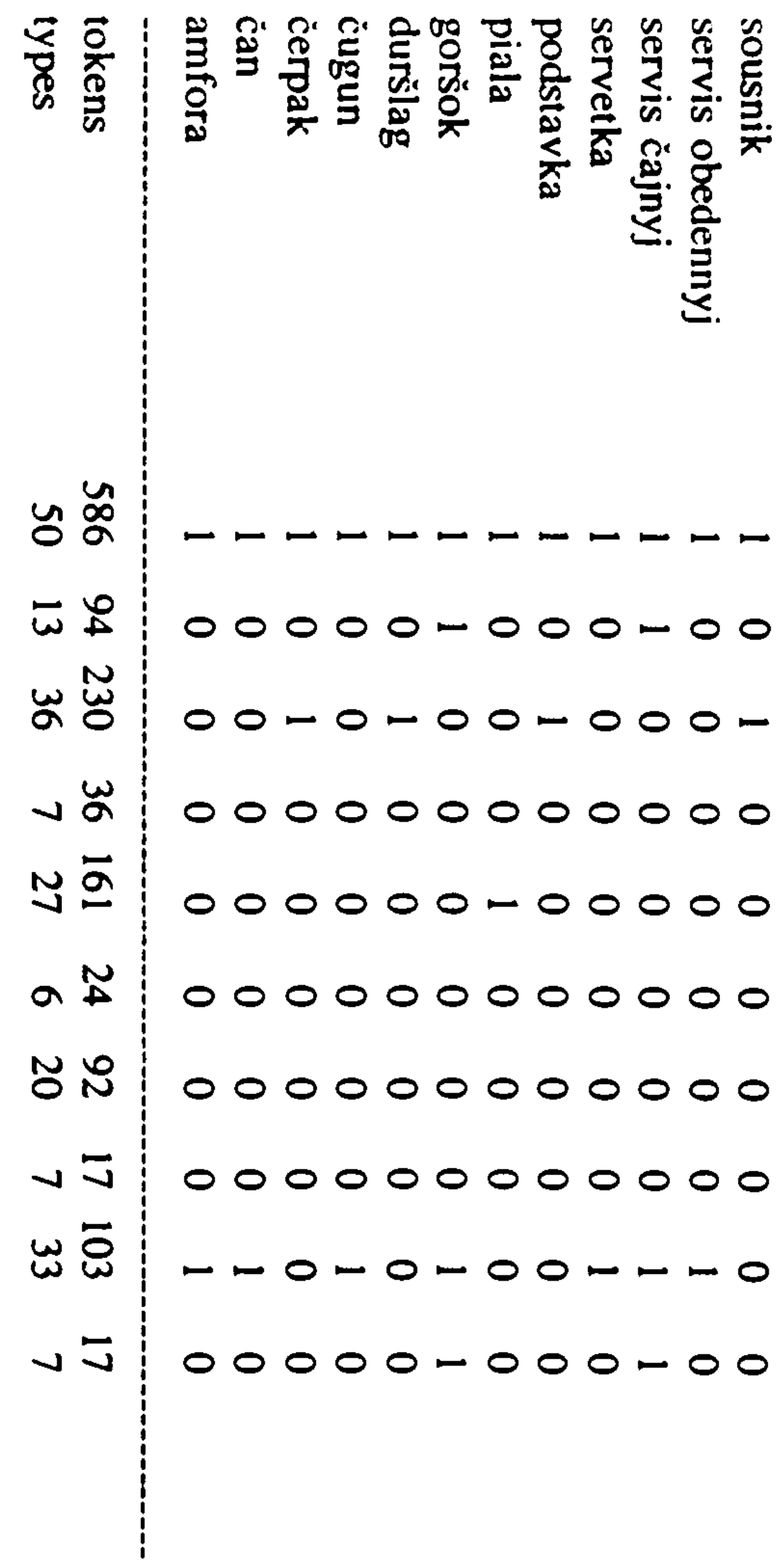


Die russische Psycholinguistin V.N. MASKADYNJA hat die Struktur 50 natürlicher Kategorien experimentell untersucht. Als Vpn dienten ihr 120 Studenten der Fakultät der Romanistik und Germanistik der Staatlichen Universität von Kalinin (Tver'); über den Anteil jedes Geschlechtes in der Gruppe der Vpn hat MASKADYNJA keine Angaben gemacht. Jede Vpn erhielt ein Heft, in dem auf fünf Seiten je zehn Kategorien aufgelistet waren. Die Reihenfolge der Kategorien in den Versuchsunterlagen sollte eine gegenseitige Beeinflussung nach Möglichkeit ausschließen, wurde jedoch offensichtlich nicht variiert. Das Experiment fand in zwei Schritten statt. Zunächst wurden die Vpn gebeten, zu jeder Kategorie einige Mitglieder zu notieren; eine Zeitbeschränkung wurde dabei nicht auferlegt. Anschließend zählten die Vpn Merkmale der Kategorie und der von ihnen genannten Mitglieder auf (MASKADYNJA 1987: 3).

Unter den 50 Kategorien, die MASKADYNJA untersucht hat, befindet sich an 24. Stelle die Kategorie ovošc̆, an 31. die Kategorie prinadležnost' dlja edy, an 33. die Kategorie ptica, an 38. die Kategorie sredstvo peredviženija und an 42. die Kategorie frukt. Die Ergebnisse sind (die Summe der tokens der Gesamtnennungen und der types der Erstnennungen wurde von uns hinzugefügt):

\begin{tabular}{|c|c|c|}
\hline OVOŠC & Gesamtnennungen & Erstnennungen \\
\hline ogurec & 75 & 28 \\
\hline pomidor & 71 & 20 \\
\hline morkov' & 64 & 13 \\
\hline kartofel' & 57 & 22 \\
\hline kapusta & 55 & 15 \\
\hline svekla & 51 & 12 \\
\hline luk & 24 & 2 \\
\hline kabačok & 19 & 0 \\
\hline baklažan & 18 & 1 \\
\hline redis, rediska & 15 & 2 \\
\hline repa & 12 & 0 \\
\hline tykva & 7 & 1 \\
\hline redka & 6 & 0 \\
\hline dynja & 5 & 2 \\
\hline perec & 5 & 0 \\
\hline arbuz & 4 & 0 \\
\hline brjukva & 2 & 1 \\
\hline gorox & 2 & 0 \\
\hline patisson & 2 & 0 \\
\hline česnok & 2 & 0 \\
\hline bazar & 1 & 1 \\
\hline ogorod & 1 & 0 \\
\hline
\end{tabular}




\begin{tabular}{lrr} 
pole & 1 & 0 \\
ukrop & 1 & 0 \\
\hline tokens & 500 & 120 \\
types & 24 & 13
\end{tabular}

\section{PRINADLEŽNOST \\ DLJA EDY}

Gesamtnennungen

Erstnennungen

\begin{tabular}{|c|c|c|}
\hline vilka & 117 & 35 \\
\hline tarelka & 115 & 31 \\
\hline ložka & 113 & 38 \\
\hline nož & 100 & 10 \\
\hline čaška & 40 & 4 \\
\hline stakan & 16 & 0 \\
\hline bljudce & 8 & 0 \\
\hline kružka & 7 & 0 \\
\hline kastrjulja & 6 & 1 \\
\hline miska & 5 & 0 \\
\hline salfetka & 5 & 0 \\
\hline čajnaja ložka & 3 & 0 \\
\hline paločki (japonskie, kitajskie) & 2 & 0 \\
\hline povareška & 2 & 0 \\
\hline stolovaja ložka & 2 & 0 \\
\hline čajnik & 2 & 0 \\
\hline bokal & 1 & 0 \\
\hline bljudo & 1 & 0 \\
\hline lavrovyj list & 1 & 0 \\
\hline moloko & 1 & 0 \\
\hline mjaso & 1 & 0 \\
\hline ovošč & 1 & 0 \\
\hline paločki (buterbrodnye) & 1 & 0 \\
\hline piala & 1 & 0 \\
\hline podnos & 1 & 1 \\
\hline posuda & 1 & 0 \\
\hline pogreb & 1 & 0 \\
\hline
\end{tabular}


frukt

xolodil'nik

120

tokens

556

types

$29^{1}$

120

7

\section{PTICA}

Gesamtnennungen

Erstnennungen

\begin{tabular}{|c|c|c|}
\hline vorobej & 66 & 13 \\
\hline vorona & 36 & 6 \\
\hline sinica & 36 & 6 \\
\hline orel & 32 & 12 \\
\hline golub' & 27 & 10 \\
\hline solovej & 25 & 10 \\
\hline lebed' & 18 & 0 \\
\hline soroka & 16 & 4 \\
\hline žuravl' & 15 & 1 \\
\hline lastočka & 15 & 2 \\
\hline sokol & 14 & 2 \\
\hline djatel & 13 & 1 \\
\hline snegir' & 13 & 2 \\
\hline grač & 13 & 1 \\
\hline galka & 12 & 6 \\
\hline jastreb & 12 & 2 \\
\hline čajka & 11 & 5 \\
\hline kurica & 10 & 5 \\
\hline popugaj & 10 & 0 \\
\hline drozd & 9 & 0 \\
\hline skvorec & 9 & 2 \\
\hline aist & 8 & 2 \\
\hline żavoronok & 8 & 1 \\
\hline kolibri & 8 & 0 \\
\hline straus & 8 & 0 \\
\hline kukuška & 6 & 1 \\
\hline striž & 5 & 1 \\
\hline utka & 5 & 3 \\
\hline caplja & 5 & 0 \\
\hline gus' & 4 & 2 \\
\hline SC & 4 & \\
\hline
\end{tabular}

' Bei MASKADYNJA irrtümlicherweise mit 30 angegeben. 


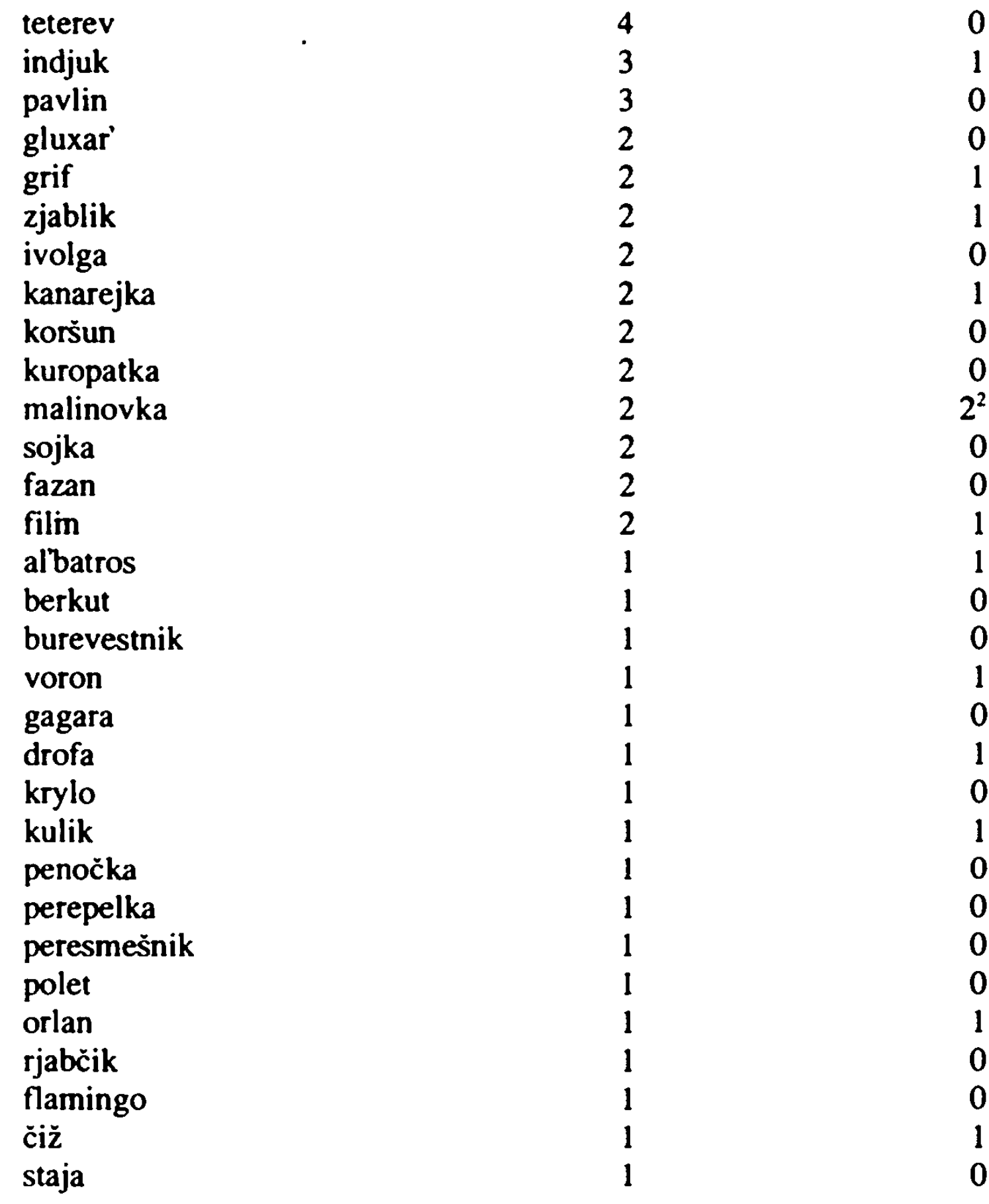

tokens

types

${ }^{2}$ Bei MASKadynja 22, was wohl Tippfehler für (2)2 ist, d.h. zwei Gesamtnennungen, die beide an erster Stelle im betreffenden Fragebogen stehen. 
SREDSTVO

PEREDVIŽENIJA Gesamtnennungen Erstnennungen

\begin{tabular}{|c|c|c|}
\hline velosiped & 71 & 25 \\
\hline samolet & 69 & 7 \\
\hline tramvaj & 52 & 17 \\
\hline avtobus & 50 & 15 \\
\hline mašina & 50 & 24 \\
\hline poezd & 49 & 2 \\
\hline avtomobil' & 38 & 23 \\
\hline trollejbus & 27 & 0 \\
\hline motocikl & 20 & 0 \\
\hline paroxod & 12 & 0 \\
\hline metro & 10 & 0 \\
\hline taksi & 10 & 0 \\
\hline teploxod & 8 & 0 \\
\hline nogi & 8 & 0 \\
\hline peškom & 6 & 1 \\
\hline samokat & 4 & 0 \\
\hline korabl’ & 4 & 0 \\
\hline kater & 3 & 0 \\
\hline lodka & 3 & 0 \\
\hline lošad’ & 3 & 0 \\
\hline telega & 3 & 0 \\
\hline èlektrička & 3 & 0 \\
\hline beg & 2 & 1 \\
\hline ezda & 2 & 0 \\
\hline koljaska & 2 & 0 \\
\hline traktor & 2 & 0 \\
\hline xodba & 2 & 1 \\
\hline aèrobus & 1 & 0 \\
\hline arba & 1 & 0 \\
\hline vertolet & 1 & 0 \\
\hline vozdušnyj śar & 1 & 0 \\
\hline diližans & 1 & 0 \\
\hline dirižabl’ & 1 & 1 \\
\hline drezina & 1 & 0 \\
\hline železnaja doroga & 1 & 0 \\
\hline kover-samolet & 1 & 0 \\
\hline kombajn & 1 & 0 \\
\hline kon'ki & 1 & 0 \\
\hline lyži & 1 & 0 \\
\hline
\end{tabular}


legkovaja mašina

$$
\text { meteor }
$$

vertolet

raketa

roliki

transport
120

$1 \quad 0$

$1 \quad 0$

$1 \quad 0$

$1 \quad 0$

100

531

$45^{4}-11$ tokens

types
Gesamtnennungen

111

97

52

43

42

33

33

26

14

12

12

11

9

6

5

5

4

3

2

2

I

1

1

1

1
Erstnennungen
84

9

0

4

4

7

1

1

1

1

0

0

2

1

2

0

1

1

0

0

0

0

0

0

0

${ }^{3}$ Hier fehlen offensichtlich drei Fragebögen.

4 Bei MASKADYNJA irrtümlicherweise mit 44 angegeben. 
196

mango

100

smorodina

$1 \quad 0$

sok

urožaj

$1 \quad 0$

jablonja

11

100

tokens

532

120

types

30

15 


\section{Bibliographie}

A. Wörterbücher und Enzyklopädien'

BASI Akademija nauk SSSR: Slovar' sovremennogo russkogo literaturnogo jazyka. Glavnaja redakcija: V. I. Černyšev (glavnyj redaktor), S. P. Obnorskij et al. Bd. 1 - 17. -- Moskau, Leningrad 1950 - 1965.

BAS2 Akademija nauk SSSR. Institut russkogo jazyka: Slovar' sovremennogo russkogo literaturnogo jazyka. Izdanie vtoroe, pererabotannoe $i$ dopolnennoe. Glavnyj redaktor K. S. Gorbačevič. Bd. 1 [A - B] - 4 [D]. -Moskau 1991 - 1993.

BSĖ Bol'šaja sovetskaja ènciklopedija. Glavnyj redaktor A. M. Proxorov. Trete izdanie. Bd. 1 - 30. -- Moskau 1970 - 1978.

BSĖ2 Boľšaja sovetskaja ènciklopedija. Glavnyj redaktor S. I. Vavilov. Vtoroe izdanie. Bd. 1 - 51. -- Moskau 1949 - 1958.

DAL' Tolkovyj slovar' živago velikoruskago jazyka Vladimira Dalja. Vtoroe izdanie, ispravlennoe i značitelno umnožennoe po rukopisi avtora. -- Bd. 1 - 4. -- Moskau, SPb. 1880 - 1882.

KĖDX Kratkaja ènciklopedija domašnego xozjajstva. Četvertoe izdanie. Redakcionnaja kollegija: A. P. Gorkin (glavnyj redaktor), I. A. Andreeva et al. -- Moskau 1993.

MAS Akademija nauk SSSR. Institut russkogo jazyka: Slovar' russkogo jazyka. Izdanie vtoroe, ispravlennoe i dopolnennoe. Glavnyj redaktor vtorogo izdanija: A. P. Evgen'eva. Bd. 1 - 4. -- Moskau 1981 - 1984.

MSÉ Malaja sovetskaja ènciklopedija. Glavnyj redaktor B. A. Vvedenskij. Trete izdanie. Bd. 1 - 10. -- Moskau 1958 - 1960.

NS Akademija nauk SSSR. Institut russkogo jazyka: Novye slova i značenija. Slovar'-spravočnik po materialam pressy i literatury 60-x godov. Pod redakciej N. Z. Kotelovoj i Ju. S. Sorokina. -- Moskau 1971.

' Diese Liste enthält nur jene Wörterbücher und Enzyklopädien, für die ein eigenes Kürzel eingeführt worden ist, da sie im Text besonders häufig zitiert werden. Alle anderen Wörterbücher werden nach Autorennamen und Erscheinungsjahr zitiert, z.B. KUZNECOVA/EFREMOVA 1986. 
Akademija nauk SSSR. Institut russkogo jazyka: Novye slova $\mathrm{i}$ značenija. Po materialam pressy i literatury $70-x$ godov. Pod redakciej N. Z. Kotelovoj. -- Moskau 1984.

ORF

Akademija nauk SSSR. Institut russkogo jazyka: Orfografičeskij slovar’ russkogo jazyka. Izdanie pjatoe, ispravlennoe i dopolnennoe. Pod redakciej S. G. Barxudarova, S. I. Ožegova i A. B. Sapiro. -- Moskau 1963.

OŽE S. I. Ožegov: Slovar’ russkogo jazyka. Izdanie četyrnadcatoe, stereotipnoe. Pod redakciej N. Ju. Svedovoj. -- Moskau 1983.

PAW I. Ja. Pawlowsky: Russisch-Deutsches Wörterbuch. Dritte, vollständig neu bearbeitete, berichtigte und vermehrte Auflage. Dritter Abdruck. -Riga, Leipzig 1923.

SĖS Sovetskij ènciklopedičeskij slovar'. Naučno-redakcionnyj sovet: A. M. Proxorov (predsedatel'), M. S. Giljarov et al. -- Moskau 1980.

SSS

Akademija nauk SSSR. Institut russkogo jazyka: Svodnyj slovar' sovremennoj russkoj leksiki. Pod redakciej R. P. Rogožnikovoj. Bd. 1 - 2. -Moskau 1991.

ŚVE S. I. Ožegov i N. Ju. Švedova: Tolkovyj slovar' russkogo jazyka. -Moskau 1992.

TIX A. N. Tixonov: Slovoobrazovatel'nyj slovar' russkogo jazyka. Bd. 1 - 2. -- Moskau 1985.

UŚA Tolkovyj slovar' russkogo jazyka. Sostavili G. O. Vinokur, B. A. Larin, S. I. Ožegov, B. V. Tomaševskij, D. N. Ušakov. Pod redakciej D. N. Ušakova. Bd. 1 - 4. -- Moskau 1935 - 1940.

VAS Russisches Etymologisches Wörterbuch. Von Max Vasmer. Bd. 1 - 3. -Heidelberg 1953 - 1958.

ZAL A. A. Zaliznjak: Grammatičeskij slovar' russkogo jazyka. Slovoizmenenie. -- Moskau 1977. 


\section{B. Sekundärliteratur}

AG-80: Akademija nauk SSSR. Institut russkogo jazyka. Russkaja grammatika. Tom 1: Fonetika, fonologija, udarenie, intonacija, slovoobrazovanie, morfologija. Tom 2: Sintaksis. Redakcionnaja kollegija: N. Ju. Śvedova et al. -- Moskau 1980.

Altmann, Gabriel (1971): Die phonologische Profilähnlichkeit -- ein Beitrag zur Typologie phonologischer Systeme der slavischen Sprachen, Phonetica 24: 922.

Altmann, Gabriel und Lehfeldt, Wemer (1980): Einführung in die quantitative Phonologie. -- Bochum [Quantitative Linguistics, vol. 7].

Al'tman, I.V. (1972): Osobennosti otglagol'nyx gnezd, Problemy strukturnoj lingvistiki 1971. Hsg. von S. K. Šaumjan. -- Moskau, pp. 245-257.

Apresjan, Jurij Derenikovič (1974): Leksičeskaja semantika. Sinonimičeskie sredstva jazyka. -- Moskau.

Apresjan, Jurij Derenikovič (ed.) (1991): Semiotika i informatika. Sbornik naučnyx statej. Vypusk 32. Materialy k Integral'nomu slovarju sovremennogo russkogo literaturnogo jazyka (obrazcy slovarnyx statej). -- Moskau.

Apresjan, Jurij Derenikovič (1992): Konnotacija kak čast' pragmatiki slova (leksikografičeskij aspekt), Russkij jazyk. Problemy grammatičeskoj semantiki $i$ ocenočnye faktory v jazyke. Vinogradovskie čtenija 19-20: 45-64.

Apresjan, Yuri D. (1992): Lexical Semantics. User's Guide to Contemporary Russian Vocabulary. -- Ann Arbor.

Apresjan, Jurij Derenikovič (1995): Izbrannye trudy. Tom I-II. -- Moskau.

Aronoff, Mark (1976): Word formation in the lexicon. -- Cambridge, Mass.

Arutjunova, N. D. (1980): K probleme funkcional'nyx tipov leksičeskogo značenija, in: N. D. Arutjunova, A. A. Ufimceva (Hsg.), Aspekty semantičeskix issledovanij. -- Moskau, pp. 156-249.

Arutjunova, Nina Davidovna (1976): Predloženie i ego smysl. Logiko-semantičeskie problemy. -- Moskau. 
Atran, Scott (1987): Ordinary Constraints on the Semantics of Living Kinds: A Commonsense Alternative to Recent Treatments of Natural-Object Terms, Mind and Language 2: 27-63.

Ax, Peter (1988): Systematik in der Biologie. Darstellung der stammesgeschichtlichen Ordnung in der lebenden Natur. -- Stuttgart.

Axmanova, Ol'ga Sergeevna (1957): Očerki po obščej i russkoj leksikologii. -Moskau.

Axmanova, Ol'ga Sergeevna (1974): Slovar' omonimov russkogo jazyka. -- Moskau.

Azarx, Julija Semenovna (1984): Slovoobrazovanie $i$ formoobrazovanie suščestvitel'nyx $v$ istorii russkogo jazyka. -- Moskau.

Barbaresi, Lavinia Merlini, Dressler, Wolfgang U. (1994): Morphopragmatics. Diminutives and Intensifiers in Italian, German, and Other Languages. -Berlin, New York.

Battig, William F. und Montague, William E. (1969): Category Norms for Verbal Items in 56 Categories: A Replication and Extension of the Connecticut Category Norms, Journal of Experimental Psychology. Monograph. Vol. 80, No. 3, Part 2.

Bauer, Laurie (1997): Derivational paradigms, Yearbook of morphology 1996, ed. by Geert Booij and Jaap van Marle. -- Dordrecht, Boston, London, pp. 243-256.

Beard, Robert (1990): The nature and origins of derivational polysemy, Lingua 81: $101-140$.

Beard, Robert (1993): Simultaneous Dual Derivation in Word Formation, Language 69: 716-741.

Beard, Robert (1995): Lexeme-Morpheme Base Morphology. -- State University of New York Press, Albany.

Becker, Thomas (1993): Morphologische Ersetzungsbildungen im Deutschen, Zeitschrift für Sprachwissenschaft 12: 185-217.

Belentschikow, Renate (1994): Komparativität und Qualität als funktional-semantische Felder im Russischen, Zeitschrift für Slawistik 39: 94-114.

Benveniste, Émile (1966): Problèmes de linguistique générale, 1. -- Paris. 
Berger, Tilman (1986): Wortbildung und Akzent im Russischen. -- München.

Berggren, Douglas (1962): The Use and Abuse of Metaphor, The Review of Metaphysics 16: 237-258, 450-472.

Berlin, Brent (1981): The Concept of Rank in Ethnobiological Classification: Some Evidence from Aguarana Folk Botany, in: Ronald W. Casson (ed.): Language, Culture and Cognition. Anthropological Perspectives. -- New York, pp. 92-113 [zuerst in American Ethnologist 3 (1976): 381-399].

Berlin, Brent (1992): Ethnobiological Classification. Principles of Categorization of Plants and Animals in Traditional Societies. -- Princeton.

Bielfeldt, Hans Holm (1982): Russisch-deutsches Wörterbuch. 14., durchgesehene Auflage. -- Berlin.

Birix, Aleksandr (1995): Metonimija v sovremennom russkom jazyke. -- München.

Birkenmaier, Willy und Mohl, Irene (1991): Russisch als Fachsprache. -- Tübingen.

Black, Max (1964): A Companion to Wittgenstein's 'Tractatus'. -- Cambridge.

Black, Max (1962): Models and Metaphors. Studies in Language and Philosophy. -Ithaca, London.

Bolinger, Dwight (1965): The Atomization of Meaning, Language 41: 555-573.

Brekle, Herbert E. und Kastovsky, Dieter (1977): Wortbildungsforschung: Entwicklung und Positionen, Perspektiven der Wortbildungsforschung. Beiträge zum Wuppertaler Wortbildungskolloquium vom 9.-10. Juli 1976. -- Bonn, pp. 7-19.

Brown, Cecil H. (1976): General principles of human anatomical partonomy and speculations on the growth of partonomic nomenclature, American Ethnologist 3: $400-424$.

Brown, R. W. (1958): How shall a thing be called? Psychological Review 65: 14-21.

Buzássyová, Klárá (1974): Sémantická štruktúra slovenských deverbativ. -- Bratislava.

Cassirer, Ernst (1988): Philosophie der symbolischen Formen. Erster Teil. Die Sprache. -- Darmstadt. 
Coleridge, Samuel Taylor (1972): The Collected Works of Samuel Taylor Coleridge. Vol. 6: Lay Sermons. Ed. by R. J. White. -- Cambridge.

Cruse, D. A. (1977): The pragmatics of lexical specificity, Journal of linguistics 15: 29-38.

Cruse, D. A. (1986): Lexical semantics. -- Cambridge.

Culioli, Antoine (1990): Pour une linguistique de l'énonciation. Opérations et représentations. Tome 1. -- Paris.

Daneš, František (1982): Zur Theorie des sprachlichen Zeichensystems, Grundlagen der Sprachkultur. Beiträge der Prager Linguistik zur Sprachtheorie und Sprachpflege. In Zusammenarbeit mit Karel Horálek und Jaroslav Kuchar herausgegeben von Jürgen Scharnhorst und Erika Ising. Teil 2. -- Berlin, pp. 132-173.

Danilenko, V. P. (1954): Obrazovanie kačestvennyx prilagatelnyx ot osnov imen suščestvitel'nyx pri pomošči suffiksov -at-, -čat- i -ast-, Trudy Instituta jazykoznanija AN SSSR 3 (1954): 120-129.

Debaty-Luca, Thierry (1985): Pour une analyse fonctionnelle des systèmes d'affixes, La linguistique 21: 221-237.

Debaty-Luca, Thierry (1986): Théorie fonctionnelle de la suffixation. (Appliquée principalement au français et au wallon du Centre). -- Paris.

Dirven, René und Paprotté, Wolf (eds.) (1985): The Ubiquity of Metaphor. Metaphor in Language and Thought. -- Amsterdam, Philadelphia.

Dokulil, Miloš (1962): Tvořeni slov v čeśtině. l. Teorie odvozováni slov. -- Prag.

Dokulil, Miloṡ (1964): Zum wechselseitigen Verhältnis zwischen Wortbildung und Syntax, Travaux linguistiques de Prague 1: 215-224.

Dokulil, Miloš (1968): Zur Theorie der Wortbildung, Wissenschaftliche Zeitschrift der Karl-Marx-Universität Leipzig. Gesellschafis- und Sprachwissenschaftliche Reihe 17: 203-211.

Doroševskij, V. [Doroszewski, W.] (1962): [Diskussionsbeitrag auf dem IV. Internationalen Slavistenkongreß], IV Meżdunarodnyj s-ezd slavistov. Materialy diskussii. Tom vtoroj. Problemy slavjanskogo jazykoznanija. -- Moskau, p. $128 \mathrm{sq}$. 
Dougherty, J. W. D. (1981): Salience and Relativity in Classification, Language, Culture, and Cognition, ed. by R. Casson. -- New York, pp. 163-180.

Dressler, Wolfgang U. (1986): Explanation in natural morphology, illustrated with comparative and agent-noun formation, Linguistics 24: 519-548.

Dubois, Danièle (ed.) (1991): Sémantique et cognition. Catégories, prototypes, typicalité. -- Paris.

Ďurovič, Ljubomir (1972): Otnositel'nye prilagatel'nye v slavjanskix jazykax (predvaritel'nye soobščenija), The Slavic Word. Proceedings of the International Slavistic Colloquium at UCLA. Edited by Dean S. Worth. -- The Hague, Paris, pp. 189-214.

Eckes, Thomas (1985): Zur internen Struktur semantischer Kategorien: Typikalitätsnormen auf der Basis von Ratings, Sprache und Kognition 1: 192-202.

Eckes, Thomas (1991): Psychologie der Begriffe. Strukturen des Wissens und Prozesse der Kategorisierung. -- Göttingen.

Eco, Umberto (1988): Sémiotique et philosophie du langage. Traduit de litalien par Myriem Bouzaher. - Paris [= Übersetzung von Semiotica e filosofia del linguaggio, Turin 1984].

Efremova, Tatjana Fedorovna (1996): Tolkovyj slovar' slovoobrazovatel'nyx edinic russkogo jazyka. -- Moskau.

Ermakova, Ol'ga Pavlovna (1984): Leksičeskie značenija proizvodnyx slov v russkom jazyke. -- Moskau.

Ermakova, Ol'ga Pavlovna und Zemskaja, Elena Andreevna (1991): K utočneniju otnošenij slovoobrazovatel'noj proizvodnosti, Russian Linguistics 15: 105-116.

Emé, Marcel (1982): Einführung in die Ordnungstheorie. -- Mannheim, Wien, Zürich.

Fanselow, Gisbert (1981): Zur Syntax und Semantik der Nominalkomposition. Ein Versuch praktischer Anwendung der Montague-Grammatik auf die Wortbildung des Deutschen. -- Tübingen.

Faust, Manfred (1978): Wortfeldstruktur und Wortverwendung, Wirkendes Wort 6: $365-401$. 
Firth, J. R. (1957): Phonological features of some Indian dialects, Papers in Linguistics. 1934-1951. -- London, pp. 47-53 [ursprünglich in Proceedings of the Second International Congress of Phonetic Sciences, 1935].

Fischer-Jørgensen, Eli (1975): Trends in phonological theory. A historical introduction. -- Kopenhagen.

Flier, Michael S. (1975): Remarks on Russian Verbal Prefixation, Slavic and East European Journal 19: 218-229.

Friedrichsdorf, Ulf und Prestel, Alexander (1985): Mengenlehre für den Mathematiker. -- Braunschweig, Wiesbaden.

Frumkina, Revekka Markovna (1985): Smysl i sxodstvo, Voprosy jazykoznanija 1985,2: 22-31.

Frumkina, Revekka Markovna und Mirkin, Boris Grigorevič: (1986): Semantika "konkretnoj" leksiki: Psixolingvističeskij podxod, Izvestija AN SSSR. Serija literatury $i$ jazyka 45,1: 12-22.

Frumkina, Revekka Markovna und Mixeev, Aleksej Vasil'evič (1985): "Svoboda" i "normativnost" $v$ èksperimentax po svobodnoj klassifikacii, Lingvističeskie $i$ psixolingvističeskie struktury reči. Hsg. von R. M. Frumkina et al. -- Moskau, pp. 66-77.

Frumkina, Revekka Markovna (1984): Cvet, smysl, sxodstvo. Aspekty psixolingvističeskogo analiza. -- Moskau.

Frumkina, Revekka Markovna, Mixeev, Aleksej Vasil'evič, Mostovaja, Anna Davidovna und Rjumina, Natalija Aleksandrovna (1991): Semantika i kategorizacija. -- Moskau.

Frumkina, Rebecca M. und Mikhejev, Alexei V. (1996): Meaning and Categorization. -- New York.

Gallant, James (1979): Russian Verbal Prefixation and Semantic Features: An Analysis of the Prefix VZ-. -- München.

Gelb, Adhémar und Goldstein, Kurt (1925): Psychologische Analysen himpathologischer Fälle. X. Über Farbennamenamnesie nebst Bemerkungen über das Wesen der amnestischen Aphasie überhaupt und die Beziehung zwischen Sprache und dem Verhalten zur Umwelt, Psychologische Forschung 6: 127-186. 
Geeraerts, Dirk (1985): Paradigm and paradox. Explorations into a paradigmatic theory of meaning and its epistemological background. -- Leuven.

Geeraerts, Dirk (1988): Where does Prototypicality Come From?, in: Brygida Rudzka-Ostyn (ed.): Topics in Cognitive Linguistics. -- Amsterdam, pp. 207229.

Geeraerts, Dirk (1989): Introduction: Prospects and problems of prototype theory, Linguistics 27: 587-612.

Gibbs, Raymond W. (1994): The poetics of mind. Figurative thought, language and understanding. -- Cambridge.

Ginzburg, E. L. und Krejdlin, G. E. (1982): Rodo-vidovye otnošenija v jazyke (taksonomičeskie operatory), Naučno-texničeskaja informacija. Serija 2, 1982, 8: 24-31.

Ginzburg, Efim Lejzerovič (1985): Konstrukcii polisemii v russkom jazyke. Taksonomija i metonimija. - Moskau.

Gipper, Helmut (1978): Denken ohne Sprache? 2. Auflage. -- Düsseldorf.

Glovinskaja, M. Ja. (1975): O zavisimosti morfemnoj členimosti slova ot stepeni ego sintagmatičeskoj frazeologizacii, Razvitie sovremennogo russkogo jazyka. 1972. Slovoobrazovanie. Ćlenimost' slova. -- Moskau, pp. 26-44.

Golanova, E. I. (1991): Puti i perspektivy izučenija slovoobrazovanija v funkcional'no-stilističeskom aspekte, Grammatičeskie issledovanija. Funkcional'no-stilističeskij aspekt. Morfologija. Slovoobrazovanie. Sintaksis. -- Moskau, pp. 93-102.

Goldstein, Kurt und Scheerer, Martin (1941): Abstract and Concrete Behavior: An Experimental Study With Special Tests, Psychological Monographs (Psychological Review, Suppl.) 53,2 [nach der Gesamtzählung: 239].

Grochowski, Maciej (1973a): Semantyczne pojęcie narzędzia -- próba interpretacji, Jezyk Polski 53 (1973): 16-22.

Grochowski, Maciej (1973b): Tekstowa realizacja semantycznego pojęcia narzędzia, Jezyk polski 53 (1973): 324-330.

Groupe M (1970): Rhétorique générale. - Paris. 
Haiman, John (1980): Dictionaries and Encyclopedias, Lingua 50: 329-357.

Hallpike, Christopher Roben (1979): The Foundations of Primitive Thought. -Oxford.

Hartenstein, Klaus (1981): Das erklärend-kombinatorische Wörterbuch im 'Smysl $\leftrightarrow$ Tekst'-Modell. -- München.

Havers, Wilhelm (1931): Handbuch der erklärenden Syntax. Ein Versuch zur Erforschung der Bedingungen und Triebkräfte in Syntax und Stilistik. -- Heidelberg.

Heine, Bernd, Ulrike Claudi and Friederike Hünnemeyer (1991): Grammaticalization. A Conceptual Framework. -- Chicago, London.

Heinz, Adam (1956): Uwagi nad funkcją znaczeniową przymiotnika odrzeczownikowego, Język Polski 36: 257-274.

Heinz, Adam (1957): Przymiotniki odrzeczownikowe w "Panu Tadeuszu", Jezyk Polski 37: 81-101.

Hjelmslev, Louis (1935/37): La catégorie des cas. Étude de grammaire générale. Bd. 1: Aarhus 1935, Bd. 2: Aarhus 1937. Ndr. München 1972.

Hjelmslev, Louis (1971): Prolegomènes à une théorie du langage. Nouvelle édition traduite du danois par Una Canger avec la collaboration d'Annick Wewer. -Paris.

Hockett, Charles F. (1954): Two Models of Grammatical Description, Word 10: 210 231.

Hoffmann, Joachim (1986): Die Welt der Begriffe. Psychologische Untersuchungen zur Organisation des menschlichen Wissens. -- Weinheim.

Holmes, Janet (1988): Sort of in New Zealand women's and men's speech, Studia Linguistica 42: 85-121.

Hudson, Richard (1990): Rez. zu Ronald W. Langacker, Foundations of Cognitive Grammar. Volume 1. Theoretical Prerequisites. - Stanford, CA 1987, in: Lingua 81: 272 - 284.

lomdin, Leonid L. (1996), Semantičeskaja nepolnota russkix imennyx paradigm, Die Welt der Slaven 41: 361-384. 
Iordanskaja, Lidija N. und Mel'čuk, Igor' A. (1980): Konnotacija v lingvističeskoj semantike, Wiener Slawistischer Almanach 6: 191-210.

Isačenko, Alexander V. (1976): Opera selecta. Russische Gegenwartssprache, russische Sprachgeschichte, Probleme der slavischen Sprachwissenschaft. -- München.

Jacenko, I. T. (1958): Formirovanie značenija slov s affiksal'noj proizvodnoj osnovoj (na materiale suščestvitel'nyx, obrazovannyx $v$ sovetskuju èpoxu), Russkij jazyk $v$ škole 19,5 (1958, September/Oktober): 17-22.

Jachnow, H. et al. (1980): Zur Erklärung und Modellierung diachroner Wortbildungsprozesse. -- München.

Jachnow, Helmut (1978): Wortbildung und ihre Modellierung anhand des serbokroatischen Verbalbereiches. -- Wiesbaden.

Jackendoff, Ray (1983): Semantics and Cognition. -- Cambridge, London.

Janda, Laura A. (1986): A Semantic Analysis of the Russian Verbal Prefixes za-, pere-, do-, and ot-. -- München.

Janko-Trinickaja, N. A. (1963): Zakonomernost' svjazej slovoobrazovatel'nogo i leksičeskogo značenij $v$ proizvodnyx slovax, Razvitie sovremennogo russkogo jazyka, ed. S. I. Ožegov, M. V. Panov. -- Moskau, pp. 83-97.

Johnson, Mark und Lakoff, George (1980): Metaphors we live by. -- Chicago, London.

Johnson, Mark (ed.) (1981): Philosophical Perspectives on Metaphor. -- Minneapolis.

Johnson, Stephen C. (1967): Hierarchical Clustering Schemes, Psychometrika 32: 241-254.

Jo Napoli, Donna, Reynolds, Bill (1994): Evaluative Affixes in Italian, Yearbook of Morphology 1994, ed. by Geert Booij and Jaap van Marle. -- Dordrecht, Boston, London, pp. 151-178.

Juravsky, Dan (1993): Universals in the Semantics of the Diminutive, Proceedings of the Nineteenth Annual Meeting of the Berkeley Linguistics Society. February 12-15, 1993. General Session and parasession on Semantic Typology and Semantic Universals. Ed. by Joshua S. Guenter, Barbara A. Kaiser, Cheryl C. Zoll. -- Ann Arbor, Michigan, pp. 423-436. 
Juravsky, Daniel (1996): Universal Tendencies in the Semantics of the Diminutive, Language 72: 533-578.

Jusupova, Nadja Gennad'evna (1980): Struktura slovoobrazovatel'nyx paradigm imen suščestvitel'nyx v sovremennom russkom jazyke. AKD. -- Moskau.

Kakridis, Ioannis (1993): Prototypensemantik und Wortbildungstheorie, Zeitschrifi für slavische Philologie 53: 113-128.

Kakridis,Yannis (im Druck): Metaphor vs. Metonymy: a Dialectical Perspective, erscheint in Quaderni di Semantica 1998, 2.

Kay, Paul (1971): Taxonomy and Semantic Contrast, Language 47: 866-887.

Keil, Frank C. (1989): Concepts, Kinds, and Cognitive Development. -- Cambridge, Mass., London.

Kempgen, Sebastian (1981): "Wortarten" als klassifikatorisches Problem der deskriptiven Grammatik. Historische und systematische Untersuchungen am Beispiel des Russischen. -- München.

Kipke, Uwe und Wille, Rudolf (1987): Formale Begriffsanalyse erläutert an einem Wortfeld, LDV-Forum 3: 31-36.

Kleiber, Georges (1993): Prototypensemantik. Eine Einführung. Übersetzt von Michael Schreiber. -- Tübingen [frz. Original: La sémantique du prototype. Catégories et sens lexical. -- Paris 1991].

Klobukova, L. P. (1981): Specifika paradigmatičeskix otnošenij v slovoobrazovanii (na materiale slovoobrazovatel'noj paradigmatiki russkix prilagatelnyx), Vestnik MGU. Serija 9. Filologija 1981,6: 19-27.

Klockow, Reinhard (1980): Linguistik der Gänsefüßchen. Untersuchung zum Gebrauch von Anführungszeichen im gegenwärtigen Deutsch. -- Frankfurt a. M.

Kolde, Gottfried (1989): Probleme der Beschreibung von sog. Heckenausdrücken im allgemeinen einsprachigen Wörterbuch, in: Franz Josef Hausmann, Oskar Reichmann, Herbert Ernst Wiegand, Ladislav Zgusta (Hsgg.): Wörterbücher. Dictionaries. Dictionnaires. Ein internationales Handbuch zur Lexikographie. Bd. 1. -- Berlin, New York, pp. 855-862. 
Krasil'nikova, E. V. (1989): K sootnošeniju slovoobrazovanija i slovoizmenenija, Problemy strukturnoj lingvistiki. 1985-1987. Otvetstvennyj redaktor V. P. Grigor'ev. -- Moskau, pp. 27-40.

Kronasser, Heinz (1968): Handbuch der Semasiologie. 2. Auflage. -- Heidelberg.

Kubrjakova, E. S. und Soboleva, P. A. (1979): O ponjatii paradigmy $v$ formoobrazovanii i slovoobrazovanii, Lingvistika i poètika. Ed. V. P. Grigor'ev. -Moskau, pp. 5-23.

Kuznecova, Ariadna Ivanovna und Efremova, Tatjana Fedorovna (1986): Slovar' morfem russkogo jazyka. - Moskau.

Labov, William (1976): Die Bedeutung von Wörtern und ihre Abgrenzbarkeit, Sprache im sozialen Kontext. Beschreibung und Erklärung struktureller und sozialer Bedeutung von Sprachvariation. Bd. 1. Herausgegeben von Norbert Dittmar und Bert-Olaf Rieck. -- Kronberg/Ts., pp. 223-254.

Lakoff, George (1972): Hedges: A Study in Meaning Criteria and the Logic of Fuzzy Concepts, Papers from the Eighth Regional Meeting. Chicago Linguistic Society. April 14-16, 1972. -- Chicago, pp. 183-228.

Lakoff, George (1987): Women, Fire, and Dangerous Things. What Categories Reveal about the Mind. -- Chicago, London.

Lévi-Strauss, Claude (1962): La pensée sauvage. -- Paris.

Lenngren, Lennart [Lönngren, Lennart] (1978): Russkie derivacionnye suffiksy. -Uppsala [= Studia Slavia Uppsaliensia 19].

Lönngren, Lennart (1981a): On Abstract Nouns in Russian and the Notion of Derivation, Scando-slavica 27: 187-197.

Lönngren, Lennart (1981b): On the Semantics of Russian Verbs Derived from Nouns, The Slavic Verb. An Anthology Presented to Hans Christian Sorensen. 16th December 1981. Hsg. von Per Jakobsen et al. -- Kopenhagen, pp. 87-95.

Lopatin, V. V. (1975): Metaforičeskaja motivacija v russkom slovoobrazovanii, Aktual'nye problemy russkogo slovoobrazovanija 1. -- Taškent, pp. 53-57.

Lopatin, Vladimir Vladimirovič (1979): K voprosu o kriterijax napravlenija slovoobrazovatelnoj motivirovannosti, Linguistische Arbeitsberichte 22: 57-67. 
Lotman, Jurij Mixajlovič (1992): Izbrannye stati $v$ trex tomax. Tom 1: Stati po semiotike i tipologii kul'tury. -- Tallinn.

Lovejoy, Arthur O. (1960): The Great Chain of Being. A Study of the History of an Idea. -- New York.

Lurija, Aleksandr Romanovič (1974): Ob istoričeskom razvitil poznavatel'nyx processov. Eksperimental'no-psixologičeskoe issledovanie. -- Moskau.

Lurija, Aleksandr Romanovič und Vygotskij, Lev Semenovič (1993): Ėtjudy po istorii povedenija. -- Moskau (Nachdruck der Originalausgabe Moskau 1930).

Malkiel, Yakov (1978): Derivational Categories, Universals of Human Language. Edited by Joseph H. Greenberg, Vol. 3. -- Stanford, California, pp. 125-149.

Mandel'štam, I. (1903): Ob umen šitel'nyx suffiksax v russkom jazyke so storony ix značenija. ( $\mathrm{K}$ istorii poètičeskago stilja), Žurnal ministerstva narodnago prosveščenija 348,7 (Ijul'): 34-66, 8 (Avgust): 317-353.

Mannhaupt, Hans-Rainer (1983): Produktionsnormen für verbale Reaktionen zu 40 geläufigen Kategorien, Sprache und Kognition 2: 264-278, Ndr. in: Willi Hager, Marcus Hasselhorn (eds.): Handbuch deutschsprachiger Wortnormen. -- Göttingen, Bern, Toronto, Seattle 1994, pp. 86-92.

Marchand, Hans (1960): The Categories and Types of Present-Day English WordFormation. A Synchronic-Diachronic Approach. 1. Auflage. -- Wiesbaden.

Marchand, Hans (1969): The Categories and Types of Present-Day English WordFormation. A Synchronic-Diachronic Approach. 2. Auflage. -- München.

Maskadynja, V. N. (1987): Normativnye dannye dlja 50 substantivnyx kategorij. $\mathrm{Na}$ materiale russkogo jazyka. -- Kalinin [Tver'] [ungedrucktes, im INION am 31.07.87 unter der Nummer 30609 deponiertes Typoskript].

Mayenowa, Maria Renata (1967): Expressions guillemetées: contribution à létude de la sémantique du texte poétique, To Honor Roman Jakobson. Bd. 2. -- The Hague, Paris, pp. 1315-1327.

Mel'čuk, Igor' Aleksandrovič (1967): K ponjatiju slovoobrazovanija, Izvestija $A N$ SSSR. Serija literatury i jazyka 26: 352-362. 
Mel'čuk, Igor' Aleksandrovič (1968): Stroenie jazykovyx znakov i vozmožnye for malno-smyslovye otnošenija meždu nimi, Izvestija AN SSSR. Serija literatury i jazyka 27: 426-438.

Mel'čuk, Igor' Aleksandrovič (1969): Ob opredelenii bol'šej/men'šej smyslovoj složnosti pri slovoobrazovatel'nyx otnošenijax, Izvestija AN SSSR. Serija literatury i jazyka 28: 126-135.

Mel'čuk, Igor' Aleksandrovič (1985): Poverxnostnyj sintaksis russkix čislovyx vyraženij. -- Wien [Wiener Slawistischer Almanach, Sonderband 16].

Melčuk, Igor’ Aleksandrovič (1993-94): Cours de morphologie générale. Vol. 1: Introduction et Première partie: Le mot. -- Montréal 1993. Vol. 2: Deuxième partie: Significations morphologiques. -- Montréal 1994.

Mel'čuk, Igor' Aleksandrovič (1995): Russkij jazyk v modeli "Smysl Tekst". -Moskau, Wien [Wiener Slawistischer Almanach, Sonderband 39].

Miller, George A. (1969): A Psychological Method to Investigate Verbal Concepts, Journal of Mathematical Psychology 6: 169-191.

Miller, George A. (1971): Empirical methods in the study of semantics, in: Semantics. An interdisciplinary reader in philosophy, linguistics and psychology. Hsgg. D. Steinberg und L. Jakobovits. -- Cambridge/Mass., pp. 569-585.

Miller, George A. (1991): The Science of Words. -- New York.

Miloslavskij, Igor' Grigor'evič (1980): Voprosy slovoobrazovatel'nogo sinteza. -Moskau.

Mjagkova, E. Ju. (1983): O nekotoryx projavlenijax kompleksnogo xaraktera èmocional'noj nagruzki slova, in: A. A. Zalevskaja (Hsg.): Psixolingvističeskie issledovanija v oblasti leksiki i fonetiki. Sbornik naučnyx trudov. -- Kalinin, pp. 99-108.

Mjagkova, E. Ju. (1986): K voprosu o specifike èmocional'noj nagruzki slova, in: A. A. Zalevskaja (Hsg.): Psixolingvističeskie problemy semantiki i ponimanija teksta. Sbornik naučnyx trudov. -- Kalinin, pp. 86-93.

Moore, George Edward (1959): Philosophical Papers. -- London.

Morkovkin, V. V. (1970): Ideografičeskie slovari. -- Moskau. 
Morris, William und Mary (1962): Dictionary of Word and Phrase Origins. -- New York, Evanston.

Mostovaja, A. D. (1990): Gipo-giperonimija i drugie semantičeskie otnošenija na konkretnyx suščestvitel'nyx, Jazyk $i$ struktura znanija. Otv. redaktor $\mathbf{R}$. $\mathbf{M}$. Frumkina. -- Moskau, 141-155.

Nikol'skaja, Evgenija Anatol'evna, Nikol'skij, Mixail Osipovič (1963): Kniga o kul'ture byta. -- Moskau.

Ortony, Andrew (ed.) (1993): Metaphor and Thought. Second edition. -- Cambridge.

Ožegov, Sergej 1. [S. O.] (1955): Kratkie zametki. 1. Sklonjaetsja li Moskva-reka?, Voprosy kul'tury reči 1: 221-224.

Panov, M. V. (1971): Ob analitičeskix prilagatel'nyx, Fonetika. Fonologija. Grammatika. K semidesjatiletiju A. A. Reformatskogo. -- Moskau, pp. 240-253.

Panov, M. V. (1968): Russkij jazyk i sovetskoe obščestvo. Sociologo-lingvističeskoe issledovanie. Pod red. M. V. Panova. Band III: Slovobrazovanie sovremennogo russkogo literaturnogo jazyka. Band IV: Morfologija i sintaksis sovremennogo russkogo literaturnogo jazyka. -- Moskau.

Pavlov, V. M. (1960): O razrjadax imen prilagatel'nyx v russkom jazyke, Voprosy jazykoznanija 1960,2: 65-70.

Polterauer, Ilona (1981): Die Deminutiva in der modernen russischen Schrifisprache. -- Wien.

Pottier, B. (1963): Recherches sur l'analyse sémantique en linguistique et en traduction mécanique. -- Nancy.

Pottier, B. (1965): La definition sémantique dans les dictionnaires, Travaux de linguistique et de littérature de l'Université de Strasbourg 3,1: 33-39.

Puškin, Aleksandr Sergeevič (1950): Polnoe sobranie sočinenij v desjati tomax. Tom vtoroj: Stixotvorenija 1820 - 1826. -- Moskau, Leningrad.

Rammelmeyer, Matthias (1983): Anmerkungen zum abgeleiteten Wort im Russischen, Studien zu Literatur und Kultur in Osteuropa. Bonner Beiträge zum 9. Internationalen Slawistenkongre $\beta$ in Kiew. Hsg. von Hans-Bernd Harder und Hans Rothe. -- Köln, Wien, pp. 215-232. 
Reiter, Norbert (1986): Die ovyj-Adjektive in Rußland. -- Berlin.

Reiter, Norbert (1988): Die Regulierung der Perspektive durch Diminutivum und Augmentativum in bulgarischen Märchentexten, Slavistische Studien zum X. Internationalen Slavistenkongreß in Sofia 1988. Hsg. von Reinhold Olesch und Hans Rothe. -- Köln, Wien, pp. 659-693.

Revzin, Isaak Iosifovič (1967): Metod modelirovanija i tipologija slavjanskich jazykov. -- Moskau.

Revzina, O. G. (1969): Struktura slovoobrazovatel'nyx polej v slavjanskix jazykax. -Moskau.

Revzina, O. G., Revzin, I. I. (1967): K postroeniju sistemy differencial'nyx priznakov dlja slovoobrazovanija suščestvitel'nyx slavjanskix jazykov, To Honor Roman Jakobson. Bd. 2. -- The Hague, Paris, pp. 1657-1666.

Rjumina, Natalija Anatol'evna (1987): Izučenie semantiki "konkretnoj" leksiki psixolingvističeskimi metodami. AKD. -- Moskau

Rogožnikova, Roza Pavlovna (1966): Varianty slov v russkom jazyke. -- Moskau.

Rosch, Eleanor (1973): On the internal structure of perceptual and semantic categories, Cognitive development and the acquisition of language, ed. by Timothy E. Moore. -- New York, San Francisco, London, pp. I11-144.

Rosch, Eleanor (1977): Human Categorization, Studies in Cross-cultural Psychology. Vol. 1, ed. by Neil Waren. -- London, New York, San Francisco, pp. 1-49.

Rosch, Eleanor und Mervis, Carolyn B. (1975): Family Resemblances: Studies in the Internal Structure of Categories, Cognitive Psychology 7: 573-605.

Rosch, Eleanor, Mervis, Carolyn B., Gray, Wayne D., Johnson, David M. und BoyesBraem, Penny (1976): Basic Objects in Natural Categories, Cognitive Psycho$\operatorname{logy} 8: 382-439$.

Rozwadowski, Jan v. (1904): Wortbildung und Wortbedeutung. -- Heidelberg.

Sarnowski, Michał (1991): Quasi-deminutywa w jezyku rosyjskim i polskim. -Wroctaw. 
Scalise, Sergio (1988): The notion of 'head' in morphology, Yearbook of Mor phology 1988, ed. by Geert Booij and Jaap van Marle. -- Dordrecht, pp. 229245.

Schmid, W. P. (1986): Eine revidierte Skizze einer allgemeinen Theorie der Wortarten, Mot et parties du discours. Word and Word Classes. Wort und Wortarten. Sous la direction de Pierre Swiggers et Willy van Hoecke. -- Louvain, pp. 85-99.

Schön, Donald A. (1993): Generative metaphor: A perspective on problem-setting in social policy, in: Ortony (ed.) (1993): 137-163.

Schupbach, Richard D. (1975): Russian Desubstantival Derivation. A paradigmatic view. -- Saratoga, California.

Schwartz, Stephen P. (ed.) (1977): Naming, Necessity, and Natural Kinds. -- Ithaca, London.

Schwartz, Stephen P. (1980): Natural kinds and nominal kinds, Mind 89: 182-195.

Schwartz, Stephen P. (1978): Putnam on Artifacts, The Philosophical Review 87: 566-574.

Simpson, G. G. (1961): Principles of Animal Taxonomy. -- New York.

Skljarevskaja, Galina Nikolaevna (1993): Metafora v sisteme jazyka. -- SPb.

Smirnickij, A. I. (1954): K voprosu o slove (problema "toždestva slova"), Trudy Instituta jazykoznanija AN SSSR 4: 3-49.

Smith, Edward E. und Medin, Douglas L. (1981): Categories and Concepts. -Cambridge (Mass.), London.

Soboleva, Polina Arkad'evna (1980): Slovoobrazovatelnaja polisemija i omonimija. -- Moskau.

Stallmann, A. (1979): Bemerkungen zum gegenseitigen Verhältnis der Wort- und Stammkomposita, Linguistische Arbeitsberichte 22: 104-110.

Sperber, Dan and Deirdre Wilson (1995): Relevance. Communication and Cognition. Second edition. -- Oxford and Cambridge, Mass. 
Stanford, W. Bedell (1936): Greek Metaphor. Studies in Theory and Practice. -Oxford.

Sussex, Roland (1974): The deep structure of adjectives in noun phrases, Journal of Linguistics 10: 111-131.

Szymanek, Bogdan (1988): Categories and Categorization in Morphology. -- Lublin.

Taylor, John R. (1989): Linguistic Categorization. Prototypes in Linguistic Theory. -- Oxford.

Tixonov, A. N. (1971): Slovoobrazovatel'nye omonimy v russkom jazyke, Russkij jazyk $v$ škole, 1971,1: 88-93.

Tixonov, Aleksandr Nikolaevič und Pardaev, Abdinazar Sajidovič (1989): Rol' gnezd odnokorennyx slov v sistemnoj organizacii russkoj leksiki. Otražennaja sinonimija. Otražennaja omonimija. Otražennaja antonimija. -- Taschkent.

Trost, Klaus (1992): Die agentivitätsstrukturelle Klassifikation der Verben und der Substantive im Polnischen (insbesondere auch des Genetiv-Akkusativs und des sogenannten Personalgenus), in: Wolfgang Eismann und Jürgen Petermann, Studia phraseologica et alia. Festschrift für Josip Matesić zum 65. Geburtstag. -- München 1992, pp. 511-529.

Tulviste, Peeter (1991): The Cultural-Historical Development of Verbal Thinking. Translated by Marie Jaroszewska Hall. -- New York.

Tversky, Amos (1977): Features of Similarity, Psychological Review 84: 327-352.

Tversky, Barbara (1986): Components and Categorization, Noun Classes and Categorization. Proceedings of a Symposium on Categorization and Noun Classification, Eugene, Oregon, October 1983. Ed. by Colette Craig. -- Amsterdam, Philadelphia, pp. 63-75.

Tversky, Barbara (1990): Where partonomies and taxonomies meet, Meanings and Prototypes. Studies in linguistic categorization, ed. by S. L. Tsohatzidis. -London, pp. 334-344.

Uluxanov, Igor' Stepanovič (1977): Slovoobrazovatel'naja semantika v russkom jazyke i principy ee opisanija. -- Moskau. 
Uluxanov, Igor' Stepanovič (1992a): Motivacija i proizvodnost' (o vozmožnosti sinxronno-diaxroničeskogo opisanija jazyka), Voprosy jazykoznanija 1992,2: 520.

Uluxanov, Igor' Stepanovič (1992b): O stepenjax slovoobrazovatel'noj motivirovannosti slov, Voprosy jazykoznanija 1992,5: 74-89.

Uluxanov, Igor Stepanovič (1996): Edinicy slovoobrazovatel'noj sistemy russkogo jazyka i ix leksičeskaja realizacija. -- Moskau.

Van Marle, Jaap (1985): On the Paradigmatic Dimension of Morphological Creativity. -- Dordrecht, Cinnaminson.

Van Marle, Jaap (1986): The domain hypothesis: the study of rival morphological processes, Linguistics 24: 601-627.

Van Schooneveld, C.H. (1978): Semantic Transmutations: Prolegomena to a Calculus of Meaning. Vol. 1: The Cardinal Semantic Structure of Prepositions, Cases, and paratacitc Conjunctions in Contemporary Standard Russian. -Bloomington.

Viehweger, Dieter et. al. (1977): Probleme der semantischen Analyse. -- Berlin.

Vilarova, Margarita (1995): Kategorialni i klassifikacionni semantični priznaci na proizvodnite leksemi s lokativno značene (vărxu material ot bălgarski, polski i ruski ezik), Săpostavitelno ezikoznanie 20, 3: 5-11.

Vinogradov, Viktor Vladimirovič (1944): O formax slova, Izvestija AN SSSR. Serija literatury $i$ jazyka 3,1: 31-44.

Vinogradova, Valentina N. (1992): Stilistika russkogo slovoobrazovanija. -- Frankfurt/M., Berlin, Bern, New York, Paris, Wien.

Vinogradova, Valentina Nikolaevna (1984): Stilističeskij aspekt russkogo slovoobrazovanija. -- Moskau.

Vinokur, G. O. (1959): Zametki po russkomu slovoobrazovaniju, in: ders., Izbrannye raboty po russkomu jazyku. -- Moskau, pp. 419-442 [ursprünglich in Izvestija AN SSSR. Otdelenie literatury i jazyka 5(1946): 315-332].

Volek. Bronislava (1987): Emotive signs in language and semantic functioning of derived nouns in Russian. -- Amsterdam, Philadelphia. 
Volockaja, Z. M. (1975): K sopostavitel'nomu opisaniju slavjanskix jazykov, Voprosy jazykoznanija 1975,5: 38-53.

Vygotskij, Lev Semenovič (1982): Myšlenie i reč, in: Lev Semenovič Vygotskij, Sobranie sočinenij v šesti tomax. Tom vtoroj: Problemy obščej psixologii. -Moskau 1982, pp. 5-361 (Originalausgabe Moskau 1934).

Wandruszka, Ulrich (1989): Rezension zu Debaty-Luca (1986), Zeitschrift für französische Sprache und Literatur 99: 194-197.

Warren, Beatrice (1988): Ambiguity and Vagueness in Adjectives, Studia Linguistica 42: $122-172$.

Weigl, Egon (1927): Zur Psychologie sogenannter Abstraktionsprozesse, Zeitschrift für Psychologie 103: 1-45, 257-322.

Wierzbicka, Anna (1972): Semantic primitives. Translated by Anna Wierzbicka and John Besemeres. -- Frankfurt.

Wierzbicka, Anna (1985): Lexicography and conceptual analysis. -- Ann Arbor.

Wille, Rudolf (1984): Liniendiagramme hierarchischer Begriffssysteme, in: H.H. Bock (Hsg.): Anwendungen der Klassifikation: Datenanalyse und numerische Klassifikation. -- Frankfurt 1984, pp. 32-51.

Wittgenstein, Ludwig (1984): Werkausgabe Band 1: Tractatus logico-philosophicus. Tagebücher 1914-1916. Philosophische Untersuchungen. -- Frankfurt.

Wittgenstein, Ludwig (1992): Werkausgabe, Band 8: Bemerkungen über die Farben. Über Gewißheit. Zettel. Vermischte Bemerkungen. -- Frankfurt.

Zemskaja, Elena Andreevna (1965): O nekotoryx faktorax razvitija slovoobrazovatel'noj sistemy sovremennogo russkogo jazyka, Problemy sovremennoj filologii. Sbornik statej $k$ semidesjatiletiju akademika $V$. V. Vinogradova. -Moskau, pp. 142-148.

Zemskaja, Elena Andreevna (1967): O semantike i sintaksičeskix svojstvax otsubstantivnyx prilagatel'nyx $\mathrm{v}$ sovremennom russkom jazyke, Istorikofilologičeskie issledovanıja. Sbornik statej k semidesjatiletiju akademika N. I. Konrada. -- Moskau, pp. 92-103.

Zemskaja, Elena Andreevna (1973): Sovremennyj russkij jazyk. Slovoobrazovanie. -Moskau. 
Zemskaja, Elena Andreevna (1975a): O ponjatii "pozicija" $\mathbf{v}$ slovoobrazovanii, Razvitie sovremennogo russkogo jazyka 1972. Slovoobrazovanie, členimost" slova. -- Moskau, pp. 14-25.

Zemskaja, Elena Andreevna (1975b): Produktivnost' i členimost', Razvitie sovremennogo russkogo literaturnogo jazyka: Slovoobrazovanie. Členimost' slova. 1972. -- Moskau, pp. 216-219.

Zemskaja, Elena Andreevna (1977): Proizvodnye slova v tolkovyx slovarjax russkogo jazyka, Sovremennaja russkaja leksikografija. 1976. -- Leningrad, pp. 110-122.

Zemskaja, Elena Andreevna (1978): O paradigmatičeskix otnošenijax v slovoobrazovanii, Russkij jazyk. Voprosy ego istorii i sovremennogo sostojanija. Vinogradovskie čtenija I-VIII. -- Moskau, pp. 63-77.

Zemskaja, Elena Andreevna (1981): Slovoobrazovanie, in: V.A. Belošapkova (ed.), Sovremennyj russkij jazyk. -- Moskau, pp. 133-239.

Zemskaja, Elena Andreevna (1984): Vidy semantičeskix otnošenij slovoobrazovatel'noj motivacii, Wiener Slawistischer Almanach 13: 337-348.

Zemskaja, Elena Andreevna (1991): Otnositel'noe prilagatel'noe kak konstruktivnyj èlement nominativnoj sistemy sovremennogo jazyka, Grammatičeskic issledovanija. Funkcional'no-stilističeskij aspekt. Morfologija. Slovoobrazovanie. Sintaksis. -- Moskau, pp. 132-164.

Zemskaja, Elena Andreevna (1992): Slovoobrazovanie kak dejatel'nost'. -- Moskau.

Ziomek, Jerzy (1990): Retoryka opisowa. -- Wrocław, Warszawa, Krakow.

Zwanenburg, Wiecher (1984): Word Formation and Meaning, Quaderni di Semantica 5: $130-142,350-365$.

Zwicky, Amold M., Sadock, Jerrold M. (1975): Ambiguity tests and how to fail them, Syntax and Semantics. Vol. 4. Edited by John P. Kimball. -- New York, San Francisco, London, pp. 1-36.

Zymner, Rüdiger (1993): Ein fremdes Wort. Zur Theorie der Metapher, Poetica 25: 3-33. 


\section{Slavistische Beiträge}

Herausgegeben von Peter Rehder

\section{5-1998}

323. Tchouboukov-Pianca, Florence: Die Konzeptualisierung der Graphomanie in der russischsprachigen postmodernen Literatur. 1995. 140 S. 34.- DM. (3-87690-594-X)

324. Iehfeldt, Werner: Einführung in die Sprachwissenschaft für Slavisten. 1995. 2. verb. und erg. Aufl. 1996. 182 S. 30.- DM. (3-87690-606-7) (Studienhilfen. 3.)

325. Bonola, Anna: Osip Mandel'stams „Egipetskaja marka“. Eine Rekonstruktion der Motivsemantik. 1995. 286 S. 46.- DM. (3-87690-607-5)

326. Бирих, Александр: Метонимия в современном русском языке. (Семантический и грамматический аспекты). 1995. II, 191 S. 40.- DM. (3-87690-608-3)

327. Schuster, Rudolf: Synonymität im Text. Eine :Untersuchung an russischen Textbeispielen. 1995. 232 S. 44.- DM. (3-87690-609-1)

328. Miszewski, Brigitta: New-Age-Diskurs in der polnischen Literaturwissenschaft. Literaturkritik und Lyrik der 70er und 80er Jahre. Rekonstruktion eines Welibildes. 1995. 236 S. 44.- DM. (3-87690-611-3)

329. Pülsch, Anja: Emigration als literarisches Verfahren bei Zinovij Zinik. 1995. 202 S. 40.- DM. (3-87690-612-1)

330. Богатырев, Константин К.: Акцентуация северолехитских говоров с исторической точки зрения. 1995. VI, 169 S. 40.- DM. (3-87690-613-X)

331. Mielke, Tomas M.: Der homosexuelle Wortschatz im Russischen. Einvernehmliche und Lagersexualität zwischen Männern. 1995. 139 S. 32.- DM. (3-87690-621-0)

332. Slavistische Linguistik 1994. Referate des XX. Konstanzer Slavistischen Arbeitstreffens, Zürich 20.-22.9. 1994. Herausgegeben von Daniel Weiss. 1995. II, 393 S. 52.- DM. (3-87690622-9)

333. Meyer, Holt: Romantische Orientierung. Wandermodelle der romantischen Bewegung (Rußland): Kjuchel'beker - Puskin - Vel'tman. 1995. 542 S. 58.- DM. (3-87690-623-7)

334. Schmaus, Alois: Lehrbuch der serbischen Sprache. Band II. Vollständig neu bearbeitet von Vera Bojić. 1996. 252 S. 36.- DM. (3-87690-624-5) (Studienhilfen. 4.)

335. Lauersdorf, Mark Richard: The question of 'Cultural Language' and interdialectal norm in 16th century Slovakia. A phonological analysis of 16th century Slovak administrative-legal texts. 1996. 267 S. 44.- DM. (3-87690-640-7)

336. Huelmann, Magdalene: Die litauischen und lettischen Arbeitslieder. Ein Vergleich. 1996. 318 S. 48.- DM. (3-87690-641-5)

337. Drews, Peter: Deutsch-slavische Literaturbeziehungen im 18. Jahrhundert. 1996. 430 S. 52.DM. (3-87690-642-3)

338. Mendoza, Imke: Zur Koordination im Russischen: $u_{a} a$ und $\partial a$ als pragmatische Konnektoren. 1996. 248 S. 44.- DM. (3-87690-648-2)

339. Eggers, Martin: Das Erzbistum des Method. Lage. Wirkung und Nachleben der kyrillomethodianischen Mission. 1996. 185 S. 40.- DM. (3-87690-649-0)

340. Maurice, Florence: Der modale Infinitiv in der modernen russischen Standardsprache. 1996. 337 S. 48.- DM. (3-87690-650-4)

341. Willich, Heide: Lev. L. Kobylinskij-Ëllis: Vom Symbolismus zur ars sacra. Eine Studie über Leben und Werk. 1996. 299 S. 48.- DM. (3-87690-651-2)

342. Slavistische Linguistik 1995. Referate des XXI. Konstanzer Slavistischen Arbeitstreffens. Mainz 26.-29.9.1995. Herausgegeben von Wolfgang Girke. 1996. II, 456 S. 56.- DM. (387690-662-8)

343. Федор Сологуб: Собрание сочинений. Том второй: Рассказы (1909-1921). Составитель Ульрих UІтельтнер. 1997. VIII. 434 S. 54.- DM. (3-87690-663-6)

344. Evans-Romaine, Karen: Boris Pasternak and the tradition of German romanticism. 1997. 329 S. 48. - DM. (3-87690-664-4)

345. Kluge, Robert: Der sowjetische Traum vom Fliegen. Analyseversuch eines gesellschaftlichen Phänomens. 1997. II, 246 S. 44.- DM. (3-87690-665-2)

346. Oppermann, Hans u.a.: Частное неофициальное письмо и тексты-рассуждения. Persönlicher Briefwechsel und Erörtenungen auf Russisch. Ein Lehr- und Ubungsheft für Fortgeschriltene. 1997. 123 S. 20.- DM. (3-87690-666-0) (Studienhilfen. 5.)

347. Sippl, Carmen: Reisetexte der russischen Moderne. Andrej Belyj und Osip Mandel'stam im Kaukausus. 1997. 283 S. 46.- DM. (3-87690-667-9)

348. Birnbaum, Henrik, Jos Schaeken: Das altkirchenslavische Wort. Bildung - Bedeutung - Herleitung. Altkirchenslavische Studien I. 1997. 190 S. 36.- DM. (3-87690-668-7) 